JOURNAL OF

SYMPLECTIC GEOMETRY

Volume 3, Number 3, 385-480, 2005

\title{
REIDEMEISTER TORSION IN FLOER-NOVIKOV THEORY AND COUNTING PSEUDO-HOLOMORPHIC TORI, II
}

\author{
YI-JEN LEE
}

This is the second part of an article in two parts, which builds the foundation of a Floer-theoretic invariant, $\boldsymbol{I}_{\mathbf{F}}$. (See [Y-J. Lee, Reidemeister torsion in Floer-Novikov theory and counting pseudoholomorphic tori, I, J. Symplectic Geom. 3 (2005), no. 2, 221-311.] for Part I). Having constructed $\boldsymbol{I}_{\mathbf{F}}$ and outlined a proof of its invariance based on bifurcation analysis in Part I, in this part we prove a series of gluing theorems to confirm the bifurcation behavior predicted in Part I. These gluing theorems are different from (and much harder than) the more conventional versions in that they deal with broken trajectories or broken orbits connected at degenerate rest points which are not Morse-Bott. The issues of orientation and signs are also settled in the last section. This part is strongly dependent on Part I, and is meant only for readers familiar with the previous part of this article.

\section{Contents}

$\begin{array}{ll}\text { 1. Overview } & 386\end{array}$

1.1. A brief summary. $\quad 386$

1.2. Basics of gluing theory. $\quad 387$

1.3. Gluing flow lines ending in degenerate critical points 401

2. Gluing at deaths I: pregluing and estimates 405

2.1. Statement of the gluing theorem. 405

2.2. The pregluing. 406

2.3. The weighted norms. $\quad 408$

2.4. The error estimate. 409

2.5. Bounding linear and nonlinear terms. 411

3. Gluing at Deaths II: the Kuranishi structure 412 
3.1. The generalized kernel and generalized cokernel.

4. Gluing at Deaths III: the gluing map 422

4.1. Understanding the Kuranishi map. 422

4.2. Surjectivity of the gluing map. 431

4.3. Gluing broken orbits. $\quad 437$

5. Gluing at births 439

5.1. Statement of the gluing theorem. 439

5.2. When $u \neq \bar{y}$. $\quad 440$

5.3. When $u=\bar{y}$. 449

6. The handleslide bifurcation $\quad 453$

6.1. Summary of results. 453

6.2. Nonequivariant perturbations on finite-cyclic covers. 454

6.3. $\Re$-truncated moduli spaces of perturbed flows. 457

7. Orientation and signs 461

7.1. Basic notions and conventions. $\quad 461$

7.2. Orienting moduli spaces. 464

7.3. The signs. 472

$\begin{array}{ll}\text { References } & 479\end{array}$

\section{Overview}

This second part forms the main technical core of the present article.

We have not attempted to make this part independent of Part I and shall frequently make use of the definitions, results, notation, and convention from Part I without repetition. Thus, we urge the reader to familiarize him/herself with Part I before attempting this one, paying particular attention to the convention in I.1.3.

References in the form of I.* shall refer to section, theorem, or equation numbers from Part I.

1.1. A brief summary. The following summarizes the results contained in this part. Recall the definitions of (RHFS*), (NEP), and admissible $(J, X)$ homotopies from Sections 4.3, 4.4, and 6.2 of [1] , respectively.

Theorem. Let $\Lambda=[1,2]$, and $\left(J^{\Lambda}, X^{\Lambda}\right)$ be an admissible $(J, X)$-homotopy connecting two regular pairs, $\left(J_{1}, X_{1}\right),\left(J_{2}, X_{2}\right)$. Then:

(1) (Corner structures of parameterized moduli spaces): The properties (RHFS2c) and (RHFS3c) hold for the CHFS generated by $\left(J^{\Lambda}, X^{\Lambda}\right)$; 
(2) (Orientation): The parameterized moduli spaces $\mathcal{M}_{P}^{\Lambda,+}$ and $\overline{\mathcal{M}}_{O}^{\Lambda, 1,+}$ may be, respectively, given coherent and grading compatible orientations such that (RHFS4) holds;

(3) (Existence of nonequivariant perturbations): (NEP) holds for all Type II handleslides in the CFHS generated by $\left(J^{\Lambda}, X^{\Lambda}\right)$.

Combining with Propositions I.4.4.6 and I.6.2.2, this completes the proof of the general invariance theorem stated in Part I, Theorem I.4.1.1.

Item (1) above follows from the gluing theorems proven in Sections $2-6$ below. Section 7 contains the discussion on orientability of the moduli spaces, the definitions of coherent and grading-compatible orientations, and as a consequence, the proof of item (2) above. Item (3) is established in Sections 6.2-6.3. There we introduce a class of (possibly nonlocal) perturbations to the induced flow on the finite-cyclic covers in the statement of (NEP), establish the expected regularity and compactness properties of the moduli spaces of such perturbed flows, and show how the arguments in the proof of Theorem I.6.2.2 may be adapted to establish the $R$-regularity of parameterized moduli spaces in this context, as required by (NEP).

Gluing theory is the unifying theme of Part II. Not only is it used repeatedly to establish the bifurcation analysis, but it also appears in the definition of coherent orientations in Section 7. Linearized versions of the gluing theorems in Sections 2-6, which actually form part of the proofs of these gluing theorems, play a major role in the verification of signs for item 2 of Theorem 1.1 above. It is for this reason that we postpone all discussion of orientations until the gluing results have been fully treated.

Thus, we begin with a quick overview of the general features of gluing theory in next subsection, then give a more specific outline of the variants contained in this article in Section 1.3.

1.2. Basics of gluing theory. This subsection gives only a minimal outline of gluing theory and its applications in Floer theory. Rather than a general account, our aim is to set up the basic framework for the proofs of the gluing theorems contained in this article and to introduce some basic notions and terminologies frequently used, some of which are not conventional. The reader may find more details and better-balanced treatments in the vast literature on this subject, for example, $[\mathbf{2}, \mathbf{3}]$ and Floer's original papers. Also, precision will sometimes be sacrificed here for the overall picture. We shall be precise in later sections, when we return to the specific context of this article.

1.2.1. The four steps of gluing theory. Gluing is useful for studying the local structure of a stratified moduli space, usually coming from compactification. Given a space of "gluing parameters" $\Xi(\mathbb{S})$ associated with a codimension $>0$ stratum $\mathbb{S}$, a typical gluing theorem constructs a map from 
$\Xi(\mathbb{S})$ to a neighborhood of $\mathbb{S}$ in the moduli space of solutions to a PDE

$$
\mathcal{F}(w)=0,
$$

which is a local diffeomorphism.

The proof of a typical gluing theorem comprises the following four major steps:

Step 1. Constructing the pregluing map and error estimates. For each gluing parameter $\chi$, one constructs an approximate solution $w_{\chi}$ to the PDE considered, which varies smoothly with $\chi$. The pregluing map $\chi \mapsto w_{\chi}$ maps the space of gluing parameters into a set in the ambient configuration space that is close to the space of solutions. An explict estimate, referred to as the "error estimate," is required to show that $\mathcal{F}\left(w_{\chi}\right)$ is sufficiently small.

Step 2. Kuranishi structure. Let $\mathfrak{D}_{w}: E \rightarrow F$ denote the linearization of $\mathcal{F}$ at $w$ (i.e., the deformation operator). This should be a Fredholm operator, and ideally, one wants to show that $\mathfrak{D}_{w_{\chi}}$ has a right inverse bounded uniformly in $\chi$. Namely, there is a $\chi$-independent constant $C_{P}>0$, and operators $P_{\chi}$ depending continuously on $\chi$, such that

$$
\mathfrak{D}_{w_{\chi}} P_{\chi}=\mathrm{id}, \quad\left\|P_{\chi}\right\| \leq C_{P} .
$$

For this to hold, judicious choices of normed spaces for $E, F$ are often called for.

Step 3. Obtaining a quadratic bound on the nonlinear part of $\mathcal{F}$, namely, (3) below. In local coordinates, one may write

$$
\mathcal{F}(w)=\mathcal{F}\left(w_{\chi}\right)+\mathfrak{D}_{w_{\chi}} \xi+N_{w_{\chi}}(\xi) \text { for } w=w_{\chi}+\xi .
$$

Setting $\xi=P_{\chi} \eta_{\chi}$, a solution to $\mathcal{F}(w)=0$ is obtained by solving

$$
\eta_{\chi}=-N_{w_{\chi}}\left(P_{\chi} \eta\right)-\mathcal{F}\left(w_{\chi}\right) .
$$

The contraction mapping theorem shows that

Lemma. Let $C_{P}$ be the upper bound on $\left\|P_{\chi}\right\|$ as above, and suppose that there is a $\chi$-independent constant $k$ such that

$$
\begin{gathered}
\left\|\mathcal{F}\left(w_{\chi}\right)\right\| \leq \frac{1}{10 k C_{P}^{2}}, \\
\left\|N_{w_{\chi}}\left(\xi_{1}\right)-N_{w_{\chi}}\left(\xi_{2}\right)\right\| \leq k\left(\left\|\xi_{1}\right\|+\left\|\xi_{2}\right\|\right)\left\|\xi_{1}-\xi_{2}\right\| \quad \forall \xi_{1}, \xi_{2} .
\end{gathered}
$$

Then there exists a unique $\eta_{\chi}$ with $\left\|\eta_{\chi}\right\| \leq 1 /\left(5 k C_{P}^{2}\right)$ solving (2). Moreover, the solution $\eta_{\chi}$ varies smoothly with $\chi$, and $\left\|\eta_{\chi}\right\| \leq 2\left\|\mathcal{F}\left(w_{\chi}\right)\right\|$.

Thus, by assigning to each gluing parameter $\chi$ the corresponding $w_{\chi}+$ $P_{\chi} \eta_{\chi}$, one obtains a smooth map from the space of gluing parameters to the moduli space. This is the gluing map. 
Step 4. Showing that the gluing map is a local diffeomorphism to a neighborhood of $S$.

1.2.2. Typical pregluing constructions in Floer theory. In Floer theory, $\mathcal{F}=\partial_{s}+\mathcal{V}$, and $\mathbb{S}$ is a stratum in a moduli space of broken trajectories or broken orbits. Thus, it is a product of reduced moduli spaces

$$
\begin{aligned}
\mathbb{S}= & \hat{\mathcal{M}}_{0} \times \hat{\mathcal{M}}_{1} \times \cdots \times \hat{\mathcal{M}}_{k}, \quad \text { or } \\
& \hat{\mathcal{M}}_{1} \times \hat{\mathcal{M}}_{2} \times \cdots \times \hat{\mathcal{M}}_{k} / \mathbb{Z} / k \mathbb{Z} .
\end{aligned}
$$

In the case of a family of Floer theories parameterized by $\Lambda, \mathbb{S}$ is a fiber product of reduced, parameterized moduli spaces over $\Lambda$

$$
\begin{aligned}
\mathbb{S}= & \hat{\mathcal{M}}_{0}^{\Lambda} \times_{\Lambda} \hat{\mathcal{M}}_{1}^{\Lambda} \times_{\Lambda} \cdots \times_{\Lambda} \hat{\mathcal{M}}_{k}^{\Lambda}, \quad \text { or } \\
& \hat{\mathcal{M}}_{1}^{\Lambda} \times_{\Lambda} \hat{\mathcal{M}}_{2}^{\Lambda} \times_{\Lambda} \cdots \times_{\Lambda} \hat{\mathcal{M}}_{k}^{\Lambda} / \mathbb{Z} / k \mathbb{Z} .
\end{aligned}
$$

The space of gluing parameters in these cases is $\Xi(\mathbb{S})=\mathbb{S} \times(\Re, \infty)^{k}$ for certain large $\Re$, and the gluing maps map into a reduced moduli space or a reduced, parameterized moduli space.

We now describe the typical pregluing construction in these situations.

Given a (unreduced) flow $u(s)$ from the critical point $x$ to $y$, we define its truncation

$$
u_{\left[-R_{-}, R+\right]}(s):= \begin{cases}u(s) & \text { when }-R_{-} / 2 \leq s \leq R_{+} / 2 \\ \exp \left(y, \beta\left(2-2 s / R_{+}\right) \eta_{y}(s)\right) & \text { when } s \geq R_{+} / 2 \\ \exp \left(x, \beta\left(2 s / R_{-}+2\right) \eta_{x}(s)\right) & \text { when } s \leq-R_{-} / 2\end{cases}
$$

where $\beta$ is a smooth cutoff function with $\beta(s)=0$ for $s \leq 0$, and $\beta(s)=1$ for $s \geq 1$, and $\eta_{y}, \eta_{x}$ are defined such that

$$
u(s)= \begin{cases}\exp \left(x, \eta_{x}(s)\right) & \text { for } s \ll-1 \\ \exp \left(y, \eta_{y}(s)\right) & \text { for } s \gg 1\end{cases}
$$

Let $u_{(-\infty, R+]}, u_{\left[-R_{-}, \infty\right)}$ be similarly defined, truncated only at the positive/negative end, respectively.

Let $\left\{\hat{u}_{0}, \hat{u}_{1}, \ldots, \hat{u}_{k}\right\}$ be a broken trajectory from $x$ to $y$, and $u_{i}$ be representatives in the respective unreduced moduli spaces. Given $\left(R_{1}, \ldots, R_{k}\right) \in \mathbb{R}_{+}^{k}$, 
we define the glued trajectory:

$$
\begin{aligned}
& u_{0} \#_{R_{1}} u_{1} \#_{R_{2}} u_{2} \cdots \#_{R_{k}} u_{k}(s):= \\
& \begin{cases}u_{0,\left(-\infty, R_{1}\right]}(s) & \text { if } s \leq R_{1} \\
\tau_{2 R_{1}} u_{1,\left[-R_{1}, R_{2}\right]}(s) & \text { if } s \in\left[R_{1}, 2 R_{1}+R_{2}\right] \\
\tau_{2 \sum i=1^{k-1} R_{i}} u_{k-1,\left[-R_{k-1}, R_{k}\right]}(s) & \vdots \\
\text { if } s \in\left[2 \sum_{i=1}^{k-2} R_{i}+R_{k-1},\right. & \left.2 \sum_{i=1}^{k-1} R_{i}+R_{k}\right] \\
\tau_{2 \sum_{i=1}^{k} R_{i}} u_{k,\left[-R_{k}, \infty\right)}(s) & \text { if } s \in\left[2 \sum_{i=1}^{k-1} R_{i}+R_{k}, \infty\right]\end{cases}
\end{aligned}
$$

where $\tau_{L}$ denotes translation by $L$ :

$$
\tau_{L} w(s):=w(s-L) .
$$

When $\left\{\hat{u}_{1}, \ldots, \hat{u}_{k}\right\}$ is a broken orbit, we may also define the glued orbit

$$
\begin{aligned}
& u_{1} \# R_{1} u_{2} \# R_{2} \cdots u_{k} \# R_{k}(s):= \\
& \left\{\begin{array}{cc}
\tau_{2 R_{1}} u_{1,\left[-R_{1}, R_{2}\right]}(s) & \text { if } s \in\left[R_{1}, 2 R_{1}+R_{2}\right] \\
\tau_{2 \sum_{i=1}^{k-1} R_{i}} u_{k-1,\left[-R_{k-1}, R_{k}\right]}(s) & \\
\text { if } s \in\left[2 \sum_{i=1}^{k-2} R_{i}+R_{k-1}, 2 \sum_{i=1}^{k-1} R_{i}+R_{k}\right] \\
\tau_{2 \sum_{i=1}^{k} R_{i}} u_{k,\left[-R_{k}, R_{1}\right]}(s) \\
\text { if } s \in\left[2 \sum_{i=1}^{k-1} R_{i}+R_{k}, 2 \sum_{i=1}^{k} R_{i}+R_{1}\right] \\
\text { for } s \in \mathbb{R} /\left(2 \sum_{i=1}^{k} R_{i}\right) \mathbb{Z} .
\end{array}\right.
\end{aligned}
$$

We shall sometimes suppress the subscript $R_{i}$ from \# when it is not important.

To define the pregluing map, in the case of broken trajectories, assign to each

$$
\chi=\left\{\hat{u}_{0}\right\} \times \cdots\left\{\hat{u}_{k}\right\} \times\left(R_{1}, \ldots, R_{k}\right) \in \hat{\mathcal{M}}_{0} \times \cdots \times \hat{\mathcal{M}}_{k} \times \mathbb{R}_{+}^{k}
$$

the $\mathbb{R}$-orbit $\hat{w}_{\chi}$ of the glued trajectory

$$
w_{\chi}=u_{0} \#_{R_{1}} u_{1} \#_{R_{2}} u_{2} \cdots \#_{R_{k}} u_{k}
$$

in the configuration space $\mathcal{B}_{P}(x, y)$, taking $u_{i}$ to be centered representatives of $\hat{u}_{i}$. Similarly for the case of broken orbits or the parameterized case. Owing to the exponential decay of flows to nondegenerate critical points, 
these constructions typically give good approximation to flow lines when the connecting rest points in the broken trajectory are nondegenerate. In this article, they are used for handleslide bifurcations and in the discussion of coherent orientations.

Remark. Equivalently, there is an unreduced version of the above construction, where the gluing map maps products of unreduced moduli spaces to an unreduced moduli space. Namely, take the space of gluing parameters to be an appropriate open subset

$$
\breve{\Xi}(\mathbb{S}) \subset \mathcal{M}_{0} \times \cdots \times \mathcal{M}_{k} ;
$$

and let the pregluing be given by the same formulae above, for fixed large $\left(R_{1}, \ldots, R_{k}\right)$, and not necessarily centered $u_{i}$. Notice that there is a free $\mathbb{R}^{k+1}$ action on $\breve{\Xi}(\mathbb{S})$, namely the product of translations on each factor moduli space $\mathcal{M}_{i}$, and the quotient $\breve{\Xi}(\mathbb{S}) / \mathbb{R}^{k+1}=\mathbb{S}$.

The equivalence is easily seen by observing that, given $\left(L_{0}, \cdots, L_{k}\right) \in$ $\mathbb{R}^{k+1}$, there is a unique $\left(L, R_{1}^{\prime}, \ldots, R_{k}^{\prime}\right) \in \mathbb{R} \times \mathbb{R}_{+}^{k}$, so that

$$
\begin{aligned}
& \tau_{L_{0}} u_{0} \#_{R_{1}} \tau_{L_{1}} u_{1} \#_{R_{2}} \tau_{L_{2}} u_{2} \cdots \#_{R_{k}} \tau_{L_{k}} u_{k} \text { approximates } \\
& \tau_{L}\left(u_{0} \#_{R_{1}^{\prime}} u_{1} \#_{R_{2}^{\prime}} u_{2} \cdots \#_{R_{k}^{\prime}} u_{k}(s)\right) .
\end{aligned}
$$

(They are equal if $u_{i}$ are replaced by their truncations.) Furthermore, under this identification, a diagonal $\mathbb{R}$-translation $\left(L_{0}, \ldots, L_{k}\right) \rightarrow\left(L_{0}+l, \ldots, L_{k}+\right.$ l) corresponds to an $\mathbb{R}$ translation in the first factor $\left(L, R_{1}^{\prime}, \ldots, R_{k}^{\prime}\right) \rightarrow(L+$ $\left.l, R_{1}^{\prime}, \ldots, R_{k}^{\prime}\right)$. Thus, we have a diffeomorphism

$$
\begin{aligned}
\breve{\Xi}(\mathbb{S}) / \mathbb{R} & \stackrel{\sim}{\rightarrow} \Xi(\mathbb{S}), \\
\left(\tau_{L_{0}} u_{0}, \ldots, \tau_{L_{k}} u_{k}\right) \bmod \mathbb{R} & \mapsto\left(\left\{{\hat{u_{0}}}_{1}, \ldots, \hat{u}_{k}\right\}, R_{1}^{\prime}, \ldots, R_{k}^{\prime}\right),
\end{aligned}
$$

and a commutative diagram

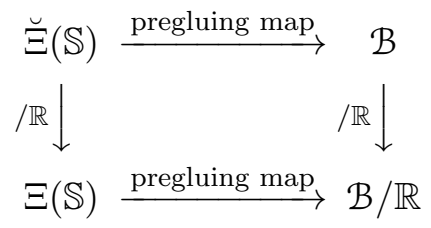

We prefer the reduced perspective in this article, because when the connecting rest points are degenerate, the (reduced) space of gluing paramaters $\Xi$ can still be described in a way similar to the above discussion, while $\breve{\Xi}$ is no longer a product of unreduced moduli spaces.

1.2.3. K-models. In general, the deformation operator might not be surjective, and the gluing theory gives a local description of the moduli space as an analytic variety in the cokernel of the deformation operator. This is the "Kuranishi model." 
For our purpose, it is convenient to introduce a linear variant of Kuranishi models, which we call "K-models." This notion of K-model will be useful both for Step 2 of the gluing procedure and in discussing the orientation issue.

Definition. A K-model for a Fredholm operator $\mathfrak{D}: E \rightarrow F$, denoted $[\mathfrak{D}: K \rightarrow C]_{B}$, or simply $[K \rightarrow C]$ when there is no danger of confusion, is a triple $K, C, B$, where $K, C$ are finite-dimensional subspaces $K \subset E, C \subset F$ respectively, and $B \subset E$ is a closed subspace such that

- $\left.\mathfrak{D}\right|_{B}: B \rightarrow \mathfrak{D}(B)$ is an isomorphism and

- there are decompositions $E=K \oplus B, F=C \oplus \mathfrak{D}(B)$ (possibly not orthogonally).

An orientation of a K-model is a choice of orientations for the spaces $K$ and $C$.

Example (Standard K-models). In this article, the "cokernel" coker $\mathfrak{D}$ refers to either the quotient space $F /$ Image( $\mathfrak{D})$ or an arbitrary subspace of $F$ complementary to Image( $\mathfrak{D})$. A trivial example of $K$-model is [D: ker $\mathfrak{D} \rightarrow$ coker $\mathfrak{D}]_{B}$, for any subspace $B \subset E$ complementary to ker $\mathfrak{D}$. Such will be called a standard K-model for $\mathfrak{D}$.

We shall call $K$ a "generalized kernel" of $\mathfrak{D}, C$ a "generalized cokernel", and $B$ a "B-space," for lack of better terminology. The honest kernel and cokernel of $\mathfrak{D}$ may be described in terms of $K$ and $C$ via the exact sequence:

$$
0 \rightarrow \operatorname{ker} \mathfrak{D} \stackrel{\Pi_{K}}{\longrightarrow} K \stackrel{\Pi_{C} \circ \mathfrak{D}}{\longrightarrow} C \rightarrow \text { coker } \mathfrak{D} \rightarrow 0
$$

where $\Pi_{K}, \Pi_{C}$ are projections with respect to the above decompositions of $E$ and $F$.

Here are some other simple examples of K-models frequently encountered in this article.

Example (K-model of a stabilization). Let $\hat{\mathfrak{D}}_{\Psi}: \mathbb{R}^{k} \oplus E \rightarrow F$ denote a finite-dimensional extension of the Fredholm map $\mathfrak{D}: E \rightarrow F$,

$$
\hat{\mathfrak{D}}_{\Psi}(\vec{r}, \xi)=\Psi(\vec{r})+\mathfrak{D} \xi,
$$

where $\Psi: \mathbb{R}^{k} \rightarrow F$ is a linear map. We call $\hat{\mathfrak{D}}_{\Psi}$ a (rank-k) stabilization of $\mathfrak{D}$.

Let $[K \rightarrow C]_{B}$ be a K-model for $\mathfrak{D}$, and

$$
\hat{K}:=\mathbb{R}^{k} \oplus K \subset \mathbb{R}^{k} \oplus E, \quad \hat{B}:=* \oplus B \subset \mathbb{R}^{k} \oplus E,
$$

where $*$ denotes the trivial vector space. Then $[\hat{K} \rightarrow C]_{\hat{B}}$ is a K-model for $\hat{\mathfrak{D}}_{\Psi}$, called the stabilization of $[K \rightarrow C]_{B}$. 
Example (Reductions of K-models). Let $[\mathfrak{D}: K \rightarrow C]_{B}$ be a K-model, and suppose that there are subspaces $Q \subset K, K^{\prime} \subset K, C^{\prime} \subset C$ such that $\left.\Pi_{C} \circ \mathfrak{D}\right|_{Q}$ is injective, and $K, C$ decompose as:

$$
K=K^{\prime} \oplus Q ; \quad C=C^{\prime} \oplus \Pi_{C}(\mathfrak{D}(Q)) .
$$

Then $\left[K^{\prime} \rightarrow C^{\prime}\right]_{B^{\prime}}$ is another K-model for $\mathfrak{D}$, where $B^{\prime}=Q+B$. Such $K$-models will be called reductions (by $Q$ ) of $[K \rightarrow C]$.

Notice that if two K-models for $\mathfrak{D},\left[\mathfrak{D}: K_{1} \rightarrow C_{1}\right]_{B_{1}}$, $\left[\mathfrak{D}: K_{2} \rightarrow C_{2}\right]_{B_{2}}$ have the same B-space $B_{1}=B_{2}$, then projections of $K_{1}$ to $K_{2}$ and $C_{1}$ to $C_{2}$ (with respect to the decompositions $E=K_{1} \oplus B_{1}, F=C_{1} \oplus \mathfrak{D}\left(B_{1}\right)$ ) are isomorphisms, and vice versa. In this case, we say that the two K-models are equivalent. Two oriented K-models are said to be equivalent if they are equivalent K-models in the above sense, and the projections involved are orientation-preserving.

K-models are particularly useful in family settings. We adopt the convention of denoting a Banach space bundle over $\Lambda$ by $V^{\Lambda}$, with the fiber over $\lambda \in \Lambda$ denoted as $V_{\lambda}$. Let $\Lambda$ be a connected manifold, and $E^{\Lambda}, F^{\Lambda}$ be Banach space bundles over $\Lambda$. Let $\mathfrak{D}^{\Lambda}:=\left\{\mathfrak{D}_{\lambda} \mid \mathfrak{D}_{\lambda}: E_{\lambda} \rightarrow F_{\lambda}, \lambda \in \Lambda\right\}$ be a family of uniformly bounded Fredholm operators, continuous in operator norm. A (family) K-model for $\mathfrak{D}^{\Lambda}$, written as $\left[\mathfrak{D}^{\Lambda}: K^{\Lambda} \rightarrow C^{\Lambda}\right]_{B^{\Lambda}}$, is a triple of Banach space subbundles $K^{\Lambda} \subset E^{\Lambda}, C^{\Lambda} \subset F^{\Lambda}, B^{\Lambda} \subset E^{\Lambda}$, so that the fibers over each $\lambda \in \Lambda,\left[K_{\lambda} \rightarrow C_{\lambda}\right]_{B_{\lambda}}$ form a K-model for $\mathfrak{D}_{\lambda}$, and $\left.\mathfrak{D}_{\lambda}\right|_{B_{\lambda}}$ has a uniformly bounded left inverse.

If $\Lambda$ is finite-dimensional and compact, such K-models always exist by the Fredholmness of the family $\mathfrak{D}^{\Lambda}$. In contrast, $\bigcup_{\lambda} \operatorname{ker} \mathfrak{D}_{\lambda}, \bigcup_{\lambda}$ coker $\mathfrak{D}_{\lambda}$ may not form bundles as the dimensions of the kernels and cokernels may jump with $\lambda$.

Two K-models $\left[D_{1}: K_{1} \rightarrow C_{1}\right]_{B_{1}},\left[D_{2}: K_{2} \rightarrow C_{2}\right]_{B_{2}}$ are said to be correlated via the family $\mathrm{K}$-model $\left[D^{\Lambda}: K^{\Lambda} \rightarrow C^{\Lambda}\right]_{B^{\Lambda}}$ if they may be identified with two fibers $\left[D_{\lambda_{1}}: K_{\lambda_{1}} \rightarrow C_{\lambda_{1}}\right]_{B_{\lambda_{1}}},\left[D_{\lambda_{2}}: K_{\lambda_{2}} \rightarrow C_{\lambda_{2}}\right]_{B_{\lambda_{2}}}$ over $\lambda_{1}, \lambda_{2} \in \Lambda$. They are said to be equivalent via the family K-model if they are equivalent to two fibers. Finally, the notions of correlation and equivalence for oriented $\mathrm{K}$-models are obtained by inserting the adjective "oriented" before every mention of K-model or family K-model in the above paragraph.

Example. If two Fredholm operators $\mathfrak{D}, \mathfrak{D}^{\prime}$ are close in operator norm, one may always include them in a family

$$
\mathfrak{D}^{\Lambda}=\left\{\mathfrak{D}_{\lambda} \mid\left\|\mathfrak{D}-\mathfrak{D}_{\lambda}\right\|<\varepsilon\right\} \quad \text { for an } \varepsilon \ll 1 .
$$

Any K-model [D: $K \rightarrow C]_{B}$ may be extended into a family K-model for $\mathfrak{D}^{\Lambda}$, with trivial $B^{\Lambda}=B \times \Lambda$. In this case, we shall refer to the equivalence of K-models for $\mathfrak{D}, \mathfrak{D}^{\prime}$ without specifying the family K-model — a family Kmodel of the above description will be implied. Moreover, if $\mathfrak{D}$ is surjective, 
and $\varepsilon$ is sufficiently small, $\left[\bigcup_{\lambda}\right.$ ker $\left.\mathfrak{D}_{\lambda} \rightarrow *\right]$ form a K-model for $\mathfrak{D}^{\Lambda}$. Thus, in this case, we shall refer to correlated orientations of ker $\mathfrak{D}$ and ker $\mathfrak{D}^{\prime}$ without further specifications.

1.2.4. Gluing operators and gluing K-models. A major motivation to introduce K-models is that, in gluing theory, generalized kernels and cokernels are typically easier to construct and work with than the honest kernels and cokernels. This subsubsection explains why.

We summarize the typical properties of the Fredholm operators appearring in Floer theories as follows. A Floer-type operator is a Fredholm operator of the form:

$$
\mathfrak{D}=\partial_{s}+A(s): E \rightarrow F, \quad \text { where }
$$

- $E=W\left(\mathbb{R}_{s} \times Y, p_{2}^{*} V\right), F=L\left(\mathbb{R}_{s} \times Y, p_{2}^{*} V\right)$ for suitable Sobolev norms $W, L$.

- $V$ is an Euclidean or hermitian bundle over the manifold $Y, \mathbb{R}_{S}$ denotes the real line parameterized by $s, p_{2}: \mathbb{R}_{s} \times Y \rightarrow Y$ denotes the projection.

- $A(s): \Gamma(Y ; V) \rightarrow \Gamma(Y ; V)$ is a first-order linear differential operator, which is surjective and $L^{2}$-self-adjoint when $|s| \gg 1$.

A stabilized Floer-type operator is a stabilization of a Floer-type operator by multiplication with compactly supported functions.

Examples. In Morse theory, $Y$ is a point. In the symplectic Floer theory considered in this article, $Y=S^{1}$, and $p_{2}^{*} V=\mathbb{R}^{2 n}$ (obtained from trivializing some $\left.u^{*} K\right) . Y$ is a 3-manifold in Seiberg-Witten or instanton Floer theories.

An ordered $k$-tuple of Floer-type operators

$$
\mathfrak{D}_{1}=\partial_{s}+A_{1}(s), \ldots, \mathfrak{D}_{k}=\partial_{s}+A_{k}(s): E \rightarrow F
$$

are said to be glueable if

- $A_{1}(s)$ is constant for large $s, A_{k}(s)$ constant for very negative $s$, and for $i=2, \ldots, k-1, A_{i}(s)$ is constant in $s$ for $|s| \gg 1$;

- $A_{i}(\infty)=A_{i+1}(-\infty)$ for $i=1, \ldots, k-1$.

Given a glueable $k+1$-tuple of Floer-type operators $\mathfrak{D}_{0}, \ldots, \mathfrak{D}_{k}$, and $k+1$ tuple of functions $\left(f_{0}, \ldots, f_{k}\right) \in E^{k}$ or $F^{k}$, we may define the glued operator $\mathfrak{D}_{0} \#_{R_{1}} \cdots \#_{R_{k}} \mathfrak{D}_{k}$ and glued function $f_{0} \#_{R_{1}} \cdots \#_{R_{k}} f_{k}$ via the same formula (4), replacing $u_{i,\left[-R_{i}, R_{i+1}\right]}$ thereby $\mathfrak{D}_{i}$ and $f_{i,\left[-R_{i}, R_{i+1}\right]}$, respectively, where $f_{i,\left[-R_{i}, R_{i+1}\right]}$ is the truncation

$$
\begin{aligned}
& f_{i,\left[-R_{i}, R_{i+1}\right]}=\beta_{\left[-R_{i}, R_{i+1}\right]}(s) f_{i}, \\
& \text { where } \beta_{\left[-R_{i}, R_{i+1}\right]}(s)=\beta\left(2 s / R_{i}+2\right) \beta\left(2-2 s / R_{i+1}\right),
\end{aligned}
$$

with $R_{0}, R_{k+1}$ understood as $-\infty, \infty$, respectively, and $\beta_{(-\infty, R]}(s):=\beta(2-$ $2 s / R), \beta_{[-R, \infty)}(s):=\beta(2 s / R+2)$. 
Let $K_{i}, i=0, \ldots, k$ be subspaces in $E$ or $F$. We denote by $K_{0} \#_{R_{1}} \cdots \#_{R_{k}} K_{k}$ the subspace of $E$ or $F$ defined by

$$
K_{0} \#_{R_{1}} \cdots \#_{R_{k}} K_{k}:=\left\{f_{0} \#_{R_{1}} \cdots \#_{R_{k}} f_{k} \mid f_{i} \in K_{i}, i=0, \ldots, k\right\} .
$$

In parallel, let $\mathfrak{D}_{1}=\partial_{s}+A_{1}(s), \ldots, \mathfrak{D}_{k}=\partial_{s}+A_{k}(s)$ be a $k$-tuple of glueable Floer-type operators, with $A_{1}(-\infty)=A_{k}(\infty)$. We call such operators cyclically glueable. In this case, we may define the cyclically glued operator and functions $\mathfrak{D}_{1} \#_{R_{1}} \cdots \#_{R_{k-1}} \mathfrak{D}_{k} \#_{R_{k}}, f_{1} \#_{R_{1}} \cdots \#_{R_{k-1}} f_{k} \#_{R_{k}} \in$ $\Gamma\left(S_{2 \sum_{i} R_{i}}^{1} \times Y ; p_{2}^{*} V\right)$ via formula (5), with similar modifications. The subspace $K_{1} \#_{R_{1}} \cdots \#_{R_{k-1}} K_{k} \#_{R_{k}} \subset \Gamma\left(S_{2 \sum_{i} R_{i}}^{1} \times Y ; p_{2}^{*} V\right)$ may also be similarly defined. Furthermore, gluing and cyclic-gluing extend in an obvious way to stabilized Floer-type operators.

We denote by $\iota_{\#}^{j} K_{j}$ the subspace

$$
\begin{aligned}
\iota_{\#}^{j} K_{j}= & * \#_{R_{1}} \cdots * \#_{R_{j-1}} K_{j} \#_{R_{j}} * \cdots \#_{R_{k-1}} * \subset K_{1} \#_{R_{1}} \cdots \#_{R_{k-1}} K_{k} \\
& \text { or } * \#_{R_{1}} \cdots * \#_{R_{j-1}} K_{j} \#_{R_{j}} * \cdots * \#_{R_{k}} \subset K_{1} \#_{R_{1}} \cdots \#_{R_{k-1}} K_{k} \#_{R_{k}}
\end{aligned}
$$

depending on the context. Notice that when $R_{1}, \ldots, R_{k}$ are sufficiently large and the subspaces $K_{j}$ are finite-dimensional, then $\iota_{\#}^{j}$ are injective for all $j$.

Given $f \in \Gamma\left(\mathbb{R} \times Y ; p_{2}^{*} V\right)$ or $\Gamma\left(S^{1} \times Y ; p_{2}^{*} V\right)$ and $c \in \mathbb{R}$ or $S^{1}$, let

$$
\operatorname{res}_{c}(f):=\left.f\right|_{\{c\} \times Y} .
$$

For a subspace $K \subset \Gamma\left(\mathbb{R} \times Y ; p_{2}^{*} V\right)$ or $\Gamma\left(S^{1} \times Y ; p_{2}^{*} V\right)$, let $\operatorname{res}_{c} K$ denote the subspace $\left\{\left.f\right|_{\{c\} \times Y} \mid f \in K\right\} \subset \Gamma(Y ; V)$.

Lemma (Glued K-models). Let $\mathfrak{D}_{1}, \ldots, \mathfrak{D}_{k}$ be a $k$-tuple of glueable Floertype operators, and $\left[\mathfrak{D}_{i}: K_{i} \rightarrow C_{i}\right]_{B_{i}}$ be K-models such that res $\left._{0}\right|_{K_{i}}: K_{i} \rightarrow$ $\operatorname{res}_{0} K_{i}$ is an isomorphism, and let $\operatorname{res}_{0} B_{i} \subset \operatorname{res}_{0} E$ be a complementary subspace to $\operatorname{res}_{0} K_{i}$. Set

$$
B_{\#}:=\left\{f \mid \operatorname{res}_{0}\left(\tau_{-2 \sum_{j=1}^{i-1} R_{j}} f\right) \in \operatorname{res}_{0} B_{i}, i=1, \ldots, k\right\} .
$$

(1a) Suppose for $\Re \gg 1$ and $\vec{R}:=\left(R_{1}, \ldots, R_{k-1}\right) \in[\Re, \infty)^{k-1}$,

$$
\mathfrak{D}_{\# \vec{R}}:=\mathfrak{D}_{1} \#_{R_{1}} \cdots \#_{R_{k-1}} \mathfrak{D}_{k} \text { is Fredholm of index } \sum_{i=1}^{k} \operatorname{ind} \mathfrak{D}_{i}, \text {. }
$$

and $K_{\# \vec{R}}:=K_{1} \#_{R_{1}} \cdots \#_{R_{k-1}} K_{k}, C_{\# \vec{R}}:=C_{1} \#_{R_{1}} \cdots \#_{R_{k-1}} C_{k}$. Then $\left[\mathfrak{D}_{\# \vec{R}}: K_{\# \vec{R}} \rightarrow C_{\# \vec{R}}\right]_{B_{\#}}$ forms a K-model. In fact, these form a family K-model for the family of operators $\left\{\mathfrak{D}_{\# \vec{R}}\right\}_{\vec{R}}$. In particular, when $\mathfrak{D}_{i}$ are surjective $\forall i$, the glued operator has a right inverse bounded uniformly in $R_{1}, \ldots, R_{k-1}$. 
(1b) The same holds for $\vec{R}:=\left(R_{1}, \ldots, R_{k}\right) \in[\Re, \infty)^{k}$,

$$
\begin{gathered}
\mathfrak{D}_{\# \vec{R}}=\mathfrak{D}_{1} \#_{R_{1}} \cdots \#_{R_{k-1}} \mathfrak{D}_{k} \#_{R_{k}}, \\
K_{\# \vec{R}}=K_{1} \#_{R_{1}} \cdots \#_{R_{k-1}} K_{k} \#_{R_{k}}, \\
C_{\# \vec{R}}=C_{1} \#_{R_{1}} \cdots \#_{R_{k-1}} C_{k} \# R_{R_{k}}
\end{gathered}
$$

if, in addition, $\mathfrak{D}_{1}, \ldots, \mathfrak{D}_{k}$ is cyclically glueable with

$$
\text { ind } \mathfrak{D}_{\# \vec{R}}=\sum_{i=1}^{k} \text { ind } \mathfrak{D}_{i} \text {. }
$$

(2) Furthermore, the projection $\Pi_{\iota_{\#}^{j} C_{j}}$ (with respect to the decomposition $\left.F=\bigoplus_{i=1}^{k} \iota_{\#}^{i} C_{i} \oplus \mathfrak{D}_{\# \vec{R}}\left(B_{\#}\right)\right)$ approximates $\Pi_{C_{j}} \circ \beta_{\left[-R_{j-1}, R_{j}\right]} \circ$ $\tau_{-2 \sum_{i=1}^{j-1} R_{i}}$, where the projection $\Pi_{C_{j}}$ is with respect to the decomposition $F=C_{j} \oplus \mathfrak{D}_{j}\left(B_{j}\right)$.

There are many other ways of choosing the B-space $B_{\#}$ for the statement of this lemma to hold [cf., e.g., [4], Proposition 9]; the one described above is that which we shall stick to for the gluing constructions in this article. Notice that with this choice of $B_{\#}$, the projection $\Pi_{\iota_{\#}^{j} K_{j}}$ (with respect to the decomposition $\left.E=\bigoplus_{i} \iota_{\#}^{i} K_{i} \oplus B_{\#}\right)$ is given by

$$
\Pi_{\iota_{\#}^{j} K_{j}}=\left(\left.\operatorname{res}_{0}\right|_{K_{j}}\right)^{-1} \circ \Pi_{\text {res }_{0} K_{j}} \circ \operatorname{res}_{0} \circ \tau_{-2 \sum_{i=1}^{j-1} R_{i}},
$$

where the projection $\Pi_{\text {res }_{0} K_{j}}$ is with respect to the decomposition $\operatorname{res}_{0} E=$ $\operatorname{res}_{0} K_{j} \oplus \operatorname{res}_{0} B_{j}$.

This gluing procedure also generalizes to family situations to construct family K-models for glued family of operators from family K-models of the family of operators to be glued.

Example (K-models of deformation operators at glued trajectories/orbits). Let $\left\{\hat{u}_{0}, \ldots, \hat{u}_{k}\right\}$ be a broken trajectory and $u_{i}$ be centered representatives of $\hat{u}_{i}$. Then

$$
E_{u_{0} \#_{R_{1}} \cdots \#_{R_{k}} u_{k}}=E_{u_{\left(-\infty,-R_{1}\right]}} \#_{R_{1}} \cdots \#_{R_{k}} E_{v_{\left[R_{k}, \infty\right)}} .
$$

When $R_{0}, \ldots, R_{k}$ are large enough, [ker $E_{u_{i}}$, coker $E_{u_{i}}$ ] is a K-model for $E_{u_{i,\left[-R_{i}, R_{i+1}\right]}}$. Furthermore, viewing ker $E_{u_{i}}$ as the solution space of the first-order linear differential equation $E_{u_{i}} \xi=0$, we see that $\left.\operatorname{res}_{0}\right|_{\text {ker } E_{u_{i}}}$ is an isomorphism. Take $B_{i}=\left\{f \mid \operatorname{res}_{0}(f) \in \operatorname{ker} E_{u_{i}}^{\perp}\right\}$. By the above lemma,

$\left[\operatorname{ker} E_{u_{0}} \#_{R_{1}} \cdots \#_{R_{k}} \text { ker } E_{u_{k}} \rightarrow \operatorname{coker} E_{u_{0}} \#_{R_{1}} \cdots \#_{R_{k}} \text { coker } E_{u_{k}}\right]_{B_{\#}}$ is a K-model for $E_{u_{0} \#_{R_{1}} \cdots \#_{R_{k}} u_{k}}$. Similarly, in the case of broken orbits, we obtain a K-model for the deformation operator at the glued orbit by 
cyclically gluing the standard K-models of the deformation operators at the component trajectories.

1.2.5. Proof by contradiction and excision for right-invertibility. Though Lemma 1.2.4 above is standard, we shall include a proof here, since it showcases the typical arguments for establishing the (uniform) rightinvertibility of $\mathfrak{D}_{w_{\chi}}$ required by Step 2 of gluing. In simple situations, one may construct by excision a right inverse to $\mathfrak{D}_{w_{\chi}}$ from right inverses of the deformation operators $\mathfrak{D}_{u_{i}}$ associated to the gluing parameter $\chi$ [see, e.g., $\mathbf{2 , 3 , 5 ]}$. In more intricate situations, such as those frequently encountered in this article, it is often convenient to use an indirect, nonconstructive method, which we refer to as "proof by contradiction." This method starts by choosing a codimension ind $\mathfrak{D}_{w_{\chi}}$ subspace $B_{\chi} \subset E$. By the Fredholmness of $\mathfrak{D}_{w_{\chi}}$, if $\left.\mathfrak{D}_{w_{\chi}}\right|_{B_{\chi}}$ is injective, then $\mathfrak{D}_{w_{\chi}}$ has a bounded right inverse $P_{\chi}: F \rightarrow B_{\chi}$. Suppose otherwise that there is a sequence of unit length $\xi_{\chi} \in B_{\chi}$, such that $\mathfrak{D}_{w_{\chi}} \xi_{\chi} \rightarrow 0$. One then shows that this is impossible by estimating $\left\|\xi_{\chi}\right\|$ in terms of $\left\|\mathfrak{D}_{w_{\chi}} \xi_{\chi}\right\|$, showing that the former must go to 0 as the latter does so. This estimate is usually obtained by breaking $\xi_{\chi}$ into summands $\xi_{i}$ supported in different regions and bounding the summands using the surjectivity of $\mathfrak{D}_{u_{i}}$.

Proof of Lemma 1.2.4. (1). The proofs of (1a) and (1b) are almost identical, so we shall focus on (1a). We follow the proof by contradiction framework. First, fix $\vec{R}$ and omit it from the subscripts to simplify notation. Let $\Psi_{j}: C_{j} \rightarrow F$ be the inclusion, and let $\mathfrak{D}_{\Psi_{\#}}: \bigoplus_{j} C_{j} \oplus B_{\#} \rightarrow F$ be defined by

$$
\mathfrak{D}_{\Psi_{\#}}\left(v_{1}, \ldots, v_{k}, \xi\right)=\mathfrak{D}_{\#} \xi+\Psi_{1}\left(v_{1}\right) \#_{R_{1}} \cdots \#_{R_{k-1}} \Psi_{k}\left(v_{k}\right) .
$$

Choose $R_{1}, \ldots, R_{k-1}$ to be large enough so that $\operatorname{dim} K_{\#}=\sum_{i} \operatorname{dim} K_{i}$ and $\operatorname{dim} C_{\#}=\sum_{i} \operatorname{dim} C_{i}$. We have the decomposition $E=K_{\#} \oplus B_{\#}$ by construction. To show that $\left[\mathfrak{D}_{\#}: K_{\#} \rightarrow C_{\#}\right]_{B_{\#}}$ indeed forms a K-model, it suffices to show that $\mathfrak{D}_{\Psi_{\#}}$ is surjective, in view of the definition of $K_{\#}, C_{\#}, B_{\#}$ and the index constraint (7). Since $\mathfrak{D}_{\Psi_{\#}}$ is Fredholm of index 0 , it is equivalent to show that it is injective.

Suppose the contrary that there exists $\left(v_{1}, \ldots, v_{k}, \xi\right) \in \bigoplus_{j} C_{j} \oplus B_{\#}$ with unit norm such that

$$
\mathfrak{D}_{\Psi_{\#}}\left(v_{1}, \ldots, v_{k}, \xi\right)=0 \text {. }
$$

The fact that $\left[\mathfrak{D}_{j}: K_{j} \rightarrow C_{j}\right]_{B_{j}}$ is a K-model implies that the operator

$$
\mathfrak{D}_{\Psi_{j}}: C_{j} \oplus B_{j} \rightarrow F, \quad(v, \eta) \mapsto \mathfrak{D}_{j} \eta+\Psi_{j}(v)
$$


has a bounded inverse, and hence

$$
\begin{aligned}
& \|\left(v_{j},\left(\beta_{\left[-R_{j-1}, R_{j}\right]} \circ \tau_{-2 \sum_{i=1}^{j-1} R_{i}}\right) \xi \|\right. \\
\leq & C\left\|\mathfrak{D}_{j}\left(\beta_{\left[-R_{j-1}, R_{j}\right]} \circ \tau_{-2 \sum_{i=1}^{j-1} R_{i}}\right) \xi+\Psi_{j}(v)\right\| \\
\leq & \left.C \| \beta_{\left[-R_{j-1}, R_{j}\right]} \circ \tau_{-2 \sum_{i=1}^{j-1} R_{i}}\right) \mathfrak{D}_{\Psi_{\#}}\left(v_{1}, \ldots, v_{k}, \xi\right) \| \\
& \left.\quad+C \| \beta_{\left[-R_{j-1}, R_{j}\right]}^{\prime} \tau_{-2 \sum_{i=1}^{j-1} R_{i}}\right) \xi\|+C\|\left(1-\beta_{\left[-R_{j-1}, R_{j}\right]}\right) \Psi_{j}\left(v_{j}\right) \|
\end{aligned}
$$

$\ll 1$,

using the assumption (8) for the first term, the fact that $\beta_{\left[-R_{j-1}, R_{j}\right]}^{\prime}<$ $C\left(\sum_{i} R_{i}^{-1}\right) \ll 1$ for the second term, and the facts that $\left\|\Psi_{j}\left(v_{j}\right)\right\|$ is bounded and $R_{i}$ are large for the last term. Meanwhile, observe that the supports of $\beta_{j}:=\tau_{2 \sum_{i=1}^{j-1} R_{i}} \beta_{\left[-R_{j-1}, R_{j}\right]}$ are disjoint for different $j$, and write

$$
1-\sum_{j=1}^{k} \beta_{j}=\sum_{l=1}^{k-1} \varphi_{l},
$$

where $\varphi_{l}$ is a nonnegative function supported on

$$
\left(2 \sum_{i=1}^{l-1} R_{i}-\frac{R_{l}}{2}, 2 \sum_{i=1}^{l-1} R_{i}+\frac{R_{l}}{2}\right) .
$$

Choose $R_{1}, \ldots, R_{k}$ to be large enough so that over the support of $\varphi_{l}, \mathfrak{D}_{l}=$ $\mathfrak{D}_{l+1}=\partial_{s}+A_{l}(\infty)$. Since by assumption $\partial_{s}+A_{l}(\infty)$ has a bounded inverse, we have

$$
\begin{aligned}
& \left\|\varphi_{l} \xi\right\| \\
\leq & C^{\prime}\left\|\left(\partial_{s}+A_{l}(\infty)\right)\left(\varphi_{l} \xi\right)\right\| \\
\leq & C^{\prime}\left\|\varphi_{l} \mathfrak{D}_{\Psi_{\#}}\left(v_{1}, \ldots, v_{k}, \xi\right)\right\|+C^{\prime}\left\|\varphi_{l}^{\prime} \xi\right\| \\
& +\left\|\varphi_{l} \sum_{j=l}^{l+1} \tau_{2 \sum_{i=1}^{j-1} R_{i}}\left(\beta_{\left[-R_{j-1}, R_{j}\right]} \Psi_{j}\left(v_{j}\right)\right)\right\|
\end{aligned}
$$

$\ll 1$.

Summing (9) and (10) for all $j$ and $l$, we obtain the desired contradiction that

$$
\left\|\left(v_{1}, \ldots, v_{k}, \xi\right)\right\| \leq \sum_{j}\left\|\left(v_{j}, \beta_{j} \xi\right)\right\|+\sum_{l}\left\|\varphi_{l} \xi\right\| \ll 1 .
$$

To see the assertion about family K-models, replace $\left(v_{1}, \ldots, v_{k}, \xi\right)$ above by a sequence of unit vectors $\left\{\left(v_{1}^{\nu}, \ldots, v_{k}^{\nu}, \xi^{\nu}\right)\right\}_{\nu}$ with $\left\|\mathfrak{D}_{\Psi_{\#} \vec{R}_{\nu}}\left(v_{1}^{\nu}, \ldots, v_{k}^{\nu}, \xi^{\nu}\right)\right\| \rightarrow$ 0 . This is impossible by the same estimates, since the above estimates do not depend on the specific values of $R_{1}, \ldots, R_{k-1}$. 
(2) We now switch to the excision method. Let $\chi_{j}$ be a smooth cutoff function with value 1 on the support of $\beta_{j}$ and vanishes outside the support of $\varphi_{j-1}+\varphi_{j}\left(\right.$ with $\left.\varphi_{0}:=0=: \varphi_{k}\right)$. Let $\tilde{\chi}_{l}$ be a smooth cutoff function with value 1 on the support of $\varphi_{l}$ and vanishes outside the support of $\sum_{j=l-1}^{l} \beta_{j}$. We choose these cutoff functions such that $\left|\chi_{j}^{\prime}\right|,\left|\tilde{\chi}_{l}^{\prime}\right|$ are both bounded by $\min _{i} R_{i}^{-1} / 4$ for all $j, l$. Let $\mathfrak{G}_{\Psi_{j}}=\left(g_{\Psi_{j}}, G_{\Psi_{j}}\right): F \rightarrow C_{j} \oplus B_{j}$ and $\tilde{G}_{l}: F \rightarrow E$ be the inverses of $\mathfrak{D}_{\Psi_{j}}$ and $\partial_{s}+A_{l}(\infty)$, respectively. Let $\mathfrak{G}_{\Psi_{j}}^{\tau}=\left(g_{\Psi_{j}}^{\tau}, G_{\Psi_{j}}^{\tau}\right):=$ $\left(g_{\Psi_{j}} \tau_{-2 \sum_{i=1}^{j-1} R_{i}}, \tau_{2 \sum_{i=1}^{j-1} R_{i}} G_{\Psi_{j}} \tau_{-2 \sum_{i=1}^{j-1} R_{i}}\right)$ and set $\mathfrak{G}_{\Psi_{\#}}: F \rightarrow \bigoplus_{j} C_{j} \oplus B_{\#}$ to be

$$
\mathfrak{G}_{\Psi_{\#}}=\left(g_{\Psi_{1}}^{\tau} \beta_{1}, \ldots, g_{\Psi_{k}}^{\tau} \beta_{k}, \sum_{j} \chi_{j} G_{\Psi_{j}}^{\tau} \beta_{j}+\sum_{l} \tilde{\chi}_{l} \tilde{G}_{l} \varphi_{l}\right) .
$$

A straightforward computation shows that $\mathfrak{D}_{\Psi_{\#}} \mathfrak{G}_{\Psi_{\#}}=1+\Xi$, where $\Xi$ is small in operator norm, and so the inverse of $\mathfrak{D}_{\Psi_{\#}}$ is $\mathfrak{G}_{\Psi_{\#}}(1+\Xi)^{-1}$. Now, the projection from $F$ to $\iota_{\#}^{j} C_{j}$ is given by $\Pi_{C_{j}} \mathfrak{G}_{\Psi_{\#}}(1+\Xi)^{-1}$ while the projection from $F$ to $C_{j}$ is given by $\Pi_{C_{j}} \mathfrak{G}_{\Psi_{j}}$. Claim (2) of Lemma 1.2.4 follows from comparing these two.

1.2.6. Generalizing the gluing map. Suppose the deformation operator $\mathfrak{D}_{w_{\chi}}$ has a K-model $[K \rightarrow C]$ with nontrivial $C$, the construction of gluing map in Step 3 of Section 1.2.1 may be generalized as follows.

Write in local coordinates near $w_{\chi}$ as in Section 1.2.1, and project (1) to the subspaces $\mathfrak{D}(B), C \subset F$, respectively, while decomposing

$$
\xi=P_{\chi} \eta_{\chi}+\xi_{K} \quad \text { for } \xi_{K} \in K, P_{\chi} \eta_{\chi} \in B
$$

where $P_{\chi}: \mathfrak{D}(B) \rightarrow B$ being the left inverse of $\left.\mathfrak{D}_{w_{\chi}}\right|_{B}$. We have

$$
\begin{gathered}
\eta_{\chi}+\Pi_{\mathfrak{D}(B)}\left(\mathcal{F}\left(w_{\chi}\right)+\mathfrak{D}_{w_{\chi}} \xi_{K}\right)+\Pi_{\mathfrak{D}(B)} N_{w_{\chi}}\left(\xi_{K}+P_{\chi} \eta_{\chi}\right)=0, \\
\left.\Pi_{C}\left(\mathcal{F}\left(w_{\chi}\right)+\mathfrak{D}_{w_{\chi}} \xi_{K}\right)+N_{w_{\chi}}\left(\xi_{K}+P_{\chi} \eta_{\chi}\right)\right)=0 .
\end{gathered}
$$

If $\xi_{K}$ is sufficiently small, the contraction mapping theorem (Lemma 1.2.1) applies to the first equation above to obtain a solution of $\eta_{\chi}$ depending on $\xi_{K}$. Substitute this into the second equation, we obtain a finite rank equation in $\xi_{K}$, which is itself in a finite-dimensional space. (The function on the LHS of this equation is the "Kuranishi map.") Thus, the solution space of $\xi$ is now an analytic variety in $C$. If $[K \rightarrow C]$ is a fiber of a family $\mathrm{K}$-model $\left[K^{\Xi} \rightarrow C^{\Xi}\right]$ for $\left\{\mathfrak{D}_{w_{\chi}}\right\}_{\chi \in \Xi}$, this describes the local structure of moduli space near the image of the pregluing map as an analytic variety in the finite-dimensional vector bundle $C^{\Xi} . C^{\Xi}$ is the so-called "obstruction bundle," and this is essentially the "obstruction bundle technique" pioneered by Taubes.

In general, it is difficult to understand the structure of this analytic variety. An example from this article is the case of gluing a broken trajectory or 
orbit involving $m$ Type II handleslides, where $m>1$ (cf. Section 6). According to Lemma 1.2.4, in this case the glued $\mathrm{K}$-model has an $m$-dimensional generalized cokernel. Our inability to describe the local structure of $\hat{\mathcal{M}}_{P}^{\Lambda, 1,+}$ near the stratum $T_{P, \text { hs-m }}$ or that of $\hat{\mathcal{M}}_{O}^{\Lambda, 1,+}$ near the stratum $T_{O \text {,hs-m }}$ is precisely due to the lack of understanding on the relevant analytic variety in this bundle of generalized cokernels.

1.2.7. Typical arguments for Step 4 in Floer theory. Typically, it follows directly from the discussion on Kuranishi structure in Step 2 that the gluing map is a local diffeomorphism. For example, let $\chi=\left\{\hat{u}_{0}, \ldots, \hat{u}_{k}\right\} \times$ $\left(R_{1}, \ldots, R_{k-1}\right)$, and $\check{\chi}=\left\{u_{0}\right\} \times \cdots \times\left\{u_{k}\right\}$ for corresponding representatives $u_{i}$ of $\hat{u}_{i}$ given in the Remark of Section 1.2.2. When $\hat{u}_{i}$ are all nondegenerate, Lemma 1.2.4 asserts that ker $\mathfrak{D}_{w_{\chi}}$ is isomorphic to

$$
\text { ker } \mathfrak{D}_{u_{0}} \# \cdots \# \text { ker } \mathfrak{D}_{u_{k}} \simeq T_{\bar{\chi}} \check{\Xi}(\mathbb{S}) \simeq T_{\chi} \Xi(\mathbb{S}) \times \mathbb{R} w_{\chi}^{\prime},
$$

where the first isomorphism in the above expression is the differential of the pregluing map, and the second isomorphism is due to the remark of Section 1.2.2 and the fact that $\mathfrak{D}_{L}\left(\tau_{L} w_{\chi}\right)=w_{\chi}^{\prime}$. On the other hand, the pregluing $w_{\chi}$ is close to the corresponding image of the gluing map, $w$. Thus, ker $\mathfrak{D}_{w_{\chi}} \simeq \operatorname{ker} \mathfrak{D}_{w}=T_{w} \mathcal{M}_{P}$. These together imply that the differential of the gluing map is an isomorphism from $T_{\chi} \Xi$ to $T_{w} \hat{\mathcal{M}}_{P}$.

To show that the gluing map is actually surjective to a neighborhood of $\mathbb{S}$ in $\hat{\mathcal{M}}_{P}^{+}$, one starts with the following simple consequence of the implicit function theorem.

Lemma. In the above situation, let $\mathcal{T}_{\chi} \subset T_{w_{\chi}} \mathcal{B}_{P}=E$ be the image of the differential of the pregluing map at $\chi$. Suppose the following hold for all $\chi \in \Xi:$

- $\mathcal{T}_{\chi}$, and $w_{\chi}^{\prime}$ vary smoothly with $\chi$;

- $\exists$ subspaces $B_{\chi} \subset E$ forming fibers of a bundle $B^{\Xi} \rightarrow \Xi$, such that $E$ decomposes as $E=B_{\chi} \oplus \mathcal{T}_{\chi} \oplus \mathbb{R} w_{\chi}^{\prime}$, and the projections to the summands are bounded uniformly in $\chi$.

Let $\exp \left(w_{\chi}, b_{\chi}\right) \in \mathcal{B}_{P}$ denote the element of coordinates $b_{\chi}$ in the local chart centered at $w_{\chi}$. Then there is a diffeomorphism from a small tubular neighborhood of $\{((\chi, 0), 0)\} \subset B^{\Xi} \times \mathbb{R}$ to a small tubular neighborhood, $U_{\epsilon}=\left\{\exp \left(\tau_{L} w_{\chi}, \xi\right) \mid\|\xi\|_{E}<\epsilon\right\} \subset \mathcal{B}_{P}$ defined by

$$
\left(\left(\chi, b_{\chi}\right), \tau\right) \mapsto \tau_{L}\left(\exp \left(w_{\chi}, b_{\chi}\right)\right) .
$$

In other words, $B^{\Xi}$ gives a good coordinate system of a slice of the $\mathbb{R}$-action in $U_{\epsilon}$. In our context, $\mathcal{B}_{\chi}$ is the B-space defined in Step 2, and the projection $\Pi_{B_{\chi}}=P_{\chi} \mathfrak{D}_{w_{\chi}}$. Proofs of analogous statements in the harder gauge-theoretic context, where the $\mathbb{R}$-action is replaced by the action of an 
infinite dimensional gauge group, may be found in $[\mathbf{2}$, Section $\mathbf{7 . 3}]$, and $[\mathbf{3}$, pp. 97-99].

Together with the contraction mapping theorem stated in Section 1.2.1, this lemma implies that the gluing map surjects to a tubular neighborhood (in E-norm) in the moduli space. However, the moduli space of broken trajectories is endowed with the coarser chain topology instead. Thus, a major task in Step 4 is to show that any flow line in a chain-topology neighborhood of $\mathbb{S}$ in fact lies in $U_{\epsilon}$. This requires a decay estimate of the flow lines near the connecting rest points.

In the case where the connecting rest points are nondegenerate, the relevant exponential decay estimate is akin to the decay estimate for flows ending at $y$, which has been used to derive (global) compactness of $\mathcal{M}_{P}$ from Gromov (local) compactness. Proposition 4.4 of $[\mathbf{3}]$ is recommended for a well-written account of this estimate (in the gauge-theoretic context).

1.3. Gluing flow lines ending in degenerate critical points. To verify the prediction of (RHFS2c, 3c) on the corner structure of $\hat{\mathcal{M}}_{P}^{\Lambda, 1,+}$ or $\hat{\mathcal{M}}_{O}^{\Lambda, 1,+}$ near $\mathbb{T}_{P, \mathrm{db}}, \mathbb{J}_{P}$, or $\mathbb{T}_{O, \mathrm{db}}$, one needs to glue flow lines ending at a degenerate critical point.

Now let $\left(J^{\Lambda}, X^{\Lambda}\right)$ be an admissible $(J, X)$-homotopy, and let $(0, y) \in$ $\mathcal{P}^{\Lambda, \operatorname{deg}}\left(J^{\Lambda}, X^{\Lambda}\right)$. In Sections $2-4$, we set $\mathbb{S}=\mathbb{T}_{P, \mathrm{db}}$ or $\mathbb{T}_{O, \mathrm{db}}$, which consists of broken trajectories or orbits with all the connecting rest points being $y$. In Section $5, \mathbb{S}$ is the subset in $\mathbb{J}_{P}$ consisting of connecting flow lines starting or ending in $y$. The space of gluing parameters in both cases will be $\Xi(\mathbb{S})=\mathbb{S} \times S$, where $S$ is an open interval in $\Lambda$ with left or right end 0 .

The gluing theory in these cases differ from the "standard" case outlined in Section 1.2 in many aspects. Much of the additional complication arises from the fact that, instead of the usual configuration space modeled locally on Sobolev spaces or exponentially weighted Sobolev spaces, the moduli spaces of flows to $y$ now embed in configuration spaces modeled on the polynomially weighted $W_{u}$-norm, and the deformation operator is between the $W_{u}$ and $L_{u}$ spaces introduced in Section I.5. The main difference between working with these polynomially-weighted spaces and the more commonly seen exponentially weighted ones is that the range space $L_{u}$ now has larger weights in the longitudinal direction than the domain space $W_{u}$. This often implies that all the estimates in the gluing theory need to be particularly precise in the longitudinal direction, especially near $y$, where the weight is large. Below is a quick outline of the strategies adopted in Sections 2-5.

1.3.1. Constructing pregluing. Let $\chi=\left(\left\{\hat{u}_{1}, \ldots, \hat{u}_{k}\right\}, \lambda\right) \in \Xi(\mathbb{S})$. Due to the aforementioned problem with large weights in the longitudinal direction, one needs a more delicate pregluing construction instead of the typical one explained in Section 1.2.1. 
Let $u_{\lambda, i}$ be a centered representative of $\hat{u}_{i}$ or a suitable cutoff version of it (to be specified later). Noticing that a variation in parameterization (by $s$ ) of an element in $\mathcal{B}_{P}$ or $\mathcal{B}_{O}$ gives rise to a variation of the element in the longitudinal direction, a natural solution to the above problem is to find ( $\lambda$-dependent) diffeomorphisms $\gamma_{u_{i}}: I_{i} \rightarrow \mathbb{R}$, such that

- Setting the pregluing $w_{\chi}(s, t)=u_{\lambda, i}\left(\gamma_{u_{i}}(s), t\right)$ over $I_{i} \times S^{1}$, the error $\mathcal{F}\left(w_{\chi}\right)$ projects trivially to the longitudinal direction (i.e., the direction of $\left.w_{\chi}^{\prime}\right)$ where $w_{\chi}(s, \cdot)$ is close to $y$, and $\gamma_{u_{i}}^{\prime}=1$ elsewhere.

- $s_{i}<s_{j}$ if $s_{i} \in I_{i}, s_{j} \in I_{j}$, and $i<j$, and the closures of $I_{i} \times S^{1}$ cover the domain of $w_{\chi}, \Theta$.

The above condition gives an ODE which determines $\gamma_{u_{i}}$. Furthermore, from the ODE one may derive various behaviors of $\gamma_{u_{i}}$, which will be important for the estimates throughout the proof. For instance, the length of $I_{i}$ is of order $|\lambda|^{-1 / 2}$ if $u_{i}$ is not the first or last component of a broken trajectory.

1.3.2. $\lambda$-dependent $W$-norms and partitioning of $\Theta$. In these settings, the gluing map to be constructed takes values in parameterized moduli spaces endowed with the ordinary $L_{1}^{p}$-topology. However, instead of the ordinary $L_{1}^{p}$-norms, we shall work with certain weighted norms $W_{\chi}, L_{\chi}$, because the right inverses of the deformation operator at $w_{\chi}$ is not bounded uniformly in the ordinary Sobolev norms. These weighted norms are defined similarly to the $W_{u}$-and $L_{u}$-norms in Section I.5.2, and are in some sense a combination of the $W_{u_{i}}$ - or $L_{u_{i}}$-norms of the components $u_{i}$; thus, when $u_{i}$ are all nondegenerate, the right inverse of the deformation operator at $w_{\chi}$ is expected to have a uniform bound in terms of the norms of the right inverses of the deformation operators at $u_{i}$. They are all commensurate with the usual Sobolev norms, though dependent on the gluing parameter $\chi$.

When performing estimates, we typically partition $\Theta$ into several regions depending on whether $\gamma_{u_{i}}^{\prime}$ is close to 1 and estimate over each region separately. Over the region $\Theta_{u_{i}}$, the values of $\gamma_{u_{i}}^{\prime}$ is close to 1 , and hence $w_{\chi}^{\prime}$ approximates $\partial_{\gamma} u(\gamma)$, the $W_{\chi}$-norm approximates the $W_{u_{i}}$-norm, and the deformation operator at $w_{\chi}$ may be approximated by that at $u_{i}$. The length of these regions are typically of order $|\lambda|^{-1 / 2}$ or infinite, and the estimates over these regions are similar to those in Section I.5.

In the case considered in Sections 2-4, the other regions are $\Theta_{y j}$. They have lengths of order $|\lambda|^{-1 / 2}$, and estimates over these regions often use the facts that on $\Theta_{y j}, w_{\chi}(s, \cdot)$ is close to $y$ (of distance $\leq C|\lambda|^{1 / 2}$ for some positive constant $C$ ) and that $\gamma_{u_{i}}(s)$ grows polynomially as positive multiples of $(|\lambda|(\mathfrak{l}-s))^{-1}$.

In the case considered in Section 5, the other regions are $\Theta_{y \pm}$. These are of infinite length, but $\gamma_{u}^{\prime}$ and hence also $w_{\chi}^{\prime}$ decay exponentially in the form $C_{ \pm} \exp \left(\mp \mu_{ \pm}|\lambda|^{1 / 2} s\right), C_{ \pm}, \mu_{ \pm}$being positive constants of $O(1)$. In addition to this, we also often use the fact that over this region, $w_{\chi}(s, \cdot)$ is close to 
the new critical points $y_{\lambda \pm}$ (of distance $\leq C|\lambda|^{1 / 2}$ for some positive constant $C)$ and the estimates about $y_{\lambda \pm}$ in Section I.5.3.

1.3.3. K-models. (A) Choosing the triple $K, C, B$. The deformation operators for parameterized moduli spaces are stabilizations of those for $\mathcal{M}_{P}$, $\mathcal{M}_{O}, \mathfrak{D}_{u}=E_{u}$ or $\tilde{D}_{u}$, respectively. Thus, it suffices to construct K-models for the latter. Similar to the case in Section 1.2.4, we shall always take the B-space to be $W_{\chi}^{\prime}$, the subspace of $W_{\chi}$ consisting of those $\xi$ such that $\operatorname{res}_{\gamma_{u_{i}}^{-1}(0)} \xi$ is $L_{t}^{2}$-orthogonal to $\operatorname{res}_{0}$ ker $E_{u_{i}} \forall i$. The generalized kernel will be the sum of the subspaces $\gamma_{u_{i}}^{*}$ ker $E_{u_{i}}=\left\{\gamma_{u_{i}}^{*} f \mid f \in\right.$ ker $\left.E_{u_{i}}\right\}$. The generalized cokernel is trivial in the case of Section 5 , but it is nontrivial in the case of Sections 2-4. In fact, by additivity of indices, its dimension is precisely the number of connecting rest points of the broken trajectory/orbit $\left\{\hat{u}_{1}, \ldots, \hat{u}_{k}\right\}$.

In this case, we choose the generalized cokernel to be spanned by $\left\{\mathfrak{f}_{j}\right\}$, where $\mathfrak{f}_{j}$ is a positive multiple of the product of the characteristic function of $\Theta_{y j}$ with a unit vector in the longitudinal direction. If one requires $\mathfrak{f}_{j}$ to be of unit $L_{\chi}$-norm, the $L^{\infty}$-norm of $\mathfrak{f}_{j}$ would be of order $|\lambda|^{1+1 /(2 p)}$. Heuristically, this choice is natural in the following sense.

(1) In this case, $\mathfrak{D}_{L L}$ is modeled on the operator

$$
\frac{d}{d s}: L_{1}^{p}\left(\left[\gamma_{u_{1}}^{-1}(0), \gamma_{u_{k}}^{-1}(0)\right]\right) \rightarrow L^{p}\left(\left[\gamma_{u_{1}}^{-1}(0), \gamma_{u_{k}}^{-1}(0)\right]\right)
$$

while $B=W_{\chi}^{\prime}$ models on the subspace of functions vanishing at the points $\gamma_{u_{i}}^{-1}(0)$. Thus, $\mathfrak{D}(B)$ models on the space of functions integrating to 0 on all the intervals $\left[\gamma_{u_{i}}^{-1}(0), \gamma_{u_{i+1}}^{-1}(0)\right]$. A natural choice for the complementary space $C$ is the subspace spanned by characteristic functions over these intervals.

(2) Let $\lambda, \lambda^{\prime}$ be, respectively, in a small death/birth neighborhood of 0 , $\chi=\left(\left\{\hat{u}_{1}, \ldots, \hat{u}_{k}\right\}, \lambda\right)$, and $\tilde{u}_{i} \in \mathcal{M}_{P, \lambda^{\prime}}$ be the flow line close to $u_{i}$, $\tilde{y} \in \mathcal{M}_{P, \lambda^{\prime}}$ be the short flow line from $y_{\lambda^{\prime}+}$ to $y_{\lambda^{\prime}-}$ close to the constant flow line $\bar{y}(s)=y \forall s$. Let $\tilde{y}^{\operatorname{inv}}(s):=\tilde{y}(-s)$. There is a glued trajectory or orbit $w_{\#}=\tilde{u}_{1} \# \tilde{y}^{\text {inv }} \# \tilde{u}_{2} \# \tilde{y}^{\text {inv }} \# \cdots$ that approximates $w_{\chi}$. Note that ker $E_{\tilde{y}^{\text {inv }}} \simeq$ coker $E_{\tilde{y}}$, coker $E_{\tilde{y}^{\text {inv }}} \simeq$ ker $E_{\tilde{y}}$; the former being trivial, while the latter approximates the 1-dimensional space of constant functions in the longitudinal direction (cf. Section 5.3.1 ). Thus, the glued K-model for $E_{w_{\#}}$ constructed in Example 1.2.4 also form a K-model for $E_{w_{\chi}}$, in which the general cokernel is spanned by $\left\{* \# \cdots * \#\right.$ ker $\left.E_{\tilde{y}} \# * \cdots\right\}$, which approximates $\left\{\mathfrak{f}_{j}\right\}$.

(B) Proving the isomorphism. To verify that the above choices do give rise to a desired $\mathrm{K}$-model, we need to show that the following operators are isomorphisms with uniformly bounded inverses: 
- in the case of Section $5,\left.\mathfrak{D}_{w_{\chi}}\right|_{W_{\chi}^{\prime}}: W_{\chi}^{\prime} \rightarrow L_{\chi}$,

- in the case of Sections $2-4$, the stabilization $\tilde{\mathfrak{D}}_{w_{\chi}}: \mathbb{R}^{m} \oplus W_{\chi}^{\prime} \rightarrow L_{\chi}$, $\tilde{\mathfrak{D}}_{w_{\chi}}\left(\iota_{1}, \ldots, \iota_{m}, \xi\right):=\mathfrak{D}_{w_{\chi}} \xi+\sum_{j} \iota_{j} \mathfrak{f}_{j}$.

The general outline of the proofs follows the "proof by contradiction" framework sketched in Section 1.2.5, estimating over different regions in $\Theta$ separately according to the partition outlined in Section 1.3.2 and incoporating several extra ingredients including:

- variants of Floer's lemma (cf., e.g., Lemma 3.3.1), which gives a $L^{\infty}$ bound on $|\lambda|^{-1 / 2} \xi$ over $\Theta_{y j}$. This is useful for ensuring that, in spite of the potential problem with large weights, the extra term $\beta^{\prime} \xi_{T}$ introduced by the cutoff function (as in (9)) when estimating the transversal component $\xi_{T}$ is still sufficiently small. (A different method is needed for the longitudinal component, where the problem with large weights is worse.) This estimate is also useful for bounding the $W_{\chi}$-norm of $\xi_{T}$ over $\Theta_{y j}$.

- estimates for $\iota_{j}$ and $\xi_{L}$ over $\Theta_{y j}$. In contrast to estimates over $\Theta_{u_{i}}$, the estimates over $\Theta_{y j}$ differ substantially from the stereotype exemplified by the proof of Lemma 1.2.4, especially for the longitudinal direction, since $\partial_{s}+A_{y}$ is not surjective, or even Fredholm. Since $\mathfrak{D}_{L L}$ in this region is modeled on $\partial_{s}$, a basic tool of these estimates is a simple lemma (Lemma 3.3.3) bounding the $L^{p}$-norm of a real-valued function $f$ over an interval $I$ in terms of the $\left\|f^{\prime}\right\|_{L^{p}(I)}$, the value of $f$ at an end point of $I$, and the length of $I$. The latter are in turn bounded via $\left\|\tilde{\mathfrak{D}}_{w_{\chi}}\left(\iota_{1}, \ldots, \iota_{m}, \xi\right)\right\|_{W_{\chi}}$, the vanishing of $\xi_{L}$ at the points $\gamma_{u_{i}}^{-1}(0)$, and the length estimate of $\Theta_{y j}$.

(C) Understanding the Kuranishi map. As explained above, in the case of Sections 2-4, the Kuranishi model is more interesting, as the Kuranishi map is nontrivial. To understand the Kuranishi map, one needs a better description of the projection $\Pi_{C}$. In general, this is not easy to compute when the decomposition $C \oplus \mathfrak{D}(B)$ is not orthogonal. Fortunately, due to the special property of our $\mathfrak{D}$ and our choice of $C$, there is a relatively simple way of computing $\Pi_{C}$ : very roughly speaking, modulo certain typically ignorable terms and multiplication by positive scalars, $\Pi_{\mathfrak{F}_{j}}$ is given by integrating the longitudinal component over the interval $\left[\gamma_{u_{j-1}}^{-1}(0) \gamma_{u_{j}}^{-1}(0)\right]$. (see Lemma 4.1.1 for the precise statement). Notice that this conforms with the heuristic picture sketched in item (1) of part (A) above.

1.3.4. Surjectivity of gluing map. As explained in Section 1.2.7, the main task of this step is a decay estimate for the flow line near $y$, which has to be particularly precise when $y$ is degenerate, due to the polynomially weighted norms adopted. This will be done via various refinements of the 
decay estimate in Section I.5. Given $w=\exp \left(w_{\chi}, \xi\right) \in \mathcal{M}_{P}$ in a chaintopology neighborhood of the pregluing $w_{\chi}$, we estimate the transversal and longitudinal components of $\xi$ separately. First, reparameterize $w_{\chi}$ to get $\tilde{w}$, such that the difference between $w$ and $\tilde{w}$ is transversal near $y$. This difference satisfies a differential equation which is used to obtain its pointwise estimate. On the other hand, comparing this parameterization with $\gamma_{u_{i}}$, which was used in the definition of $w_{\chi}$, one may estimate the difference between the two parameterizations via an ODE, which in turn gives a pointwise bound on the difference between $w_{\chi}$ and $\tilde{w}$ (note that this is longitudinal). The desired bound on $\|\xi\|_{W_{\chi}}$ is obtained using the transversal and longitudal pointwise estimates above.

\section{Gluing at deaths I: pregluing and estimates}

The following three sections give a detailed proof of Proposition 2.1 below, following the outline in Section 1.

This section contains the pregluing construction, the definitions of the Banach spaces as the domain and range of the relevant deformation operator, the error estimates, and estimates for the nonlinear term. Namely, Steps 1 and 3 of the gluing construction sketched in Section 1 .

2.1. Statement of the gluing theorem. The following Proposition describes the appearance of new trajectories and closed orbits near a death-birth bifurcation, by gluing broken trajectories and broken orbits at a death-birth. These trajectories all appear for $\lambda$ in a death-neighborhood; for this reason, we call this a "gluing theorem at deaths," in contrast to the gluing theorems in Section 5, where the images of the gluing maps project via $\Pi_{\Lambda}$ to birth-neighborhoods.

Proposition. Let $\left(J^{\Lambda}, X^{\Lambda}\right)$ be an admissible $(J, X)$-homotopy connecting two regular pairs, and $\mathbf{x}, \mathbf{z}$ be two path components of $\mathcal{P} \Lambda \backslash \mathcal{P} \Lambda, \operatorname{deg}$. Then:

(a) a chain-topology neighborhood of $\mathbb{T}_{P, \mathrm{db}}(\mathbf{x}, \mathbf{z} ; \Re)$ in $\hat{\mathcal{M}}_{P}^{\Lambda, 1,+}\left(\mathbf{x}, \mathbf{z} ; \mathrm{wt}_{-\langle y\rangle, e_{\mathcal{P}}} \leq \Re\right)$ is l.m.b. along $\mathbb{T}_{P, \mathrm{db}}(\mathbf{x}, \mathbf{z} ; \Re) ;$

(b) a chain-topology neighborhood of $\mathbb{T}_{O, \mathrm{db}}(\Re)$ in $\hat{\mathcal{M}}_{O}^{\Lambda, 1,+}\left(\mathrm{wt}_{-}\langle y\rangle, e_{\mathcal{P}} \leq \Re\right)$ is l.m.b. along $\mathbb{T}_{O, \mathrm{db}}(\Re)$.

Furthermore, $\Pi_{\Lambda}$ maps these neighborhoods to death-neighborhoods.

We shall focus on the proof of part (a), since the proof of part (b) is very similar: in fact, only the discussion in Section 4 on gluing maps needs slight modification. The necessary modification for part (b) will be briefly indicated in Section 4.3.

Recall that the admissibility of $\left(J^{\Lambda}, X^{\Lambda}\right)$ implies that elements in $\mathcal{P}^{\Lambda, \text { deg }}$ satisfy (RHFS1i), and lie in standard d-b neighborhoods, namely, satisfy the conditions described in Definition I.5.3.1. Thus, by possibly restricting 
to a sub-homotopy and/or reversing the orientation of $\Lambda$, we may assume without loss of generality that $\mathcal{P}^{\Lambda, \text { deg }}$ contains exactly one point, $y$, which is a death. Namely, the constant

$$
C_{y}^{\prime}>0
$$

in Definition I.5.3.1 (2b). We may also assume without loss of generality that

$$
\Pi_{\Lambda} y=0 .
$$

We now begin the construction of a gluing map from $\Xi(\mathbb{S})$ to $\hat{\mathcal{M}}_{P}^{\Lambda, 1}\left(\mathbf{x}, \mathbf{z} ; \mathrm{wt}_{-\langle y\rangle, e_{\mathcal{P}}} \leq \Re\right)$, where in this case

$$
\mathbb{S}=\mathbb{T}_{P, \mathrm{db}}(\mathbf{x}, \mathbf{z} ; \Re) ; \quad \Xi(\mathbb{S})=\mathbb{S} \times\left(0, \lambda_{0}\right) \text { for a small } \lambda_{0}>0 .
$$

As $\mathbb{T}_{P, \mathrm{db}}(\mathbf{x}, \mathbf{z} ; \Re)$ consists of finitely many isolated points, we may focus on a broken trajectory $\left\{\hat{u}_{0}, \ldots, \hat{u}_{k+1}\right\}$ in $\mathbb{T}_{P, \mathrm{db}}(\mathbf{x}, \mathbf{z} ; \Re)$. As usual, $u_{i}$ will denote the centered representative of $\hat{u}_{i}$.

2.2. The pregluing. Let $\chi:=\left(\left\{\hat{u}_{0}, \ldots, \hat{u}_{k+1}\right\}, \lambda\right) \in \mathbb{T}_{P, \mathrm{db}}(\mathbf{x}, \mathbf{z} ; \Re) \times\left(0, \lambda_{0}\right)$. Choose the representatives $u_{i}, i=0, \ldots, k+1$ such that $u_{i}(0)$ lies away from the neighborhood of $y$ mentioned in Definition I.5.3.1 (2a) and (2d). Let

$$
\delta_{\lambda} \mathcal{V}:=\mathcal{V}_{X_{\lambda}}-\mathcal{V}_{X_{0}}
$$

By Definition I.5.3.1 (2a), this is given by $\check{\theta}_{X_{\lambda}}-\check{\theta}_{X_{0}}$ when $\lambda<\lambda_{0}$ is sufficiently small. That is, when $\lambda_{0}$ is so small such that $J_{\lambda}$ is constant in $\lambda$ for $\lambda \in\left(-\lambda_{0}, \lambda_{0}\right)$. We choose $\lambda_{0}$ so that this is the case and shall simply write $J_{\lambda}=J$ for such $\lambda$.

2.2.1. Lemma. Let $\mathfrak{l}_{0}=-\infty, \mathfrak{l}_{1}=0$, and $\mathfrak{l}_{k+2}=\infty$. Then there exist $\mathfrak{l}_{i} \in \mathbb{R}, i=2, \ldots, k+1$, and homeomorphisms

$$
\gamma_{u_{i}}:\left(\mathfrak{l}_{i}, \mathfrak{l}_{i+1}\right) \rightarrow \mathbb{R} \quad \forall i \in\{0, \ldots, k+1\},
$$

so that the configuration $\underline{w}_{\chi} \in \mathcal{B}_{P}\left(x_{0}, z_{0}\right)$ defined by

$$
\underline{w}_{\chi}(s):= \begin{cases}u_{i}\left(\gamma_{u_{i}}(s)\right) & \text { for } s \in\left(\mathfrak{l}_{i}, \mathfrak{l}_{i+1}\right), i=0, \ldots, k+1 ; \\ y & \text { for } s=\mathfrak{l}_{j}, j=1, \ldots, k+1\end{cases}
$$

satisfies

$$
\begin{array}{ll}
\left\langle\underline{w}_{\chi}^{\prime}(s), \bar{\partial}_{J_{X_{\lambda}}} \underline{w}_{\chi}(s)\right\rangle_{2, t}=0 ; & \text { on } \bigcup_{i=0}^{k+1}\left[\gamma_{u_{i}}^{-1}(0), \gamma_{u_{i+1}}^{-1}(0)\right] \\
\gamma_{u_{i}}^{\prime}=1 & \text { otherwise. }
\end{array}
$$


Furthermore,

$$
\begin{gathered}
C_{0} \lambda^{-1 / 2} \leq-\gamma_{u_{0}}^{-1}(0) \leq C_{0}^{\prime} \lambda^{-1 / 2} \\
C_{i} \lambda^{-1 / 2} \leq \mathfrak{l}_{i+1}-\mathfrak{l}_{i} \leq C_{i}^{\prime} \lambda^{-1 / 2} \quad \text { for } i=1,2, \ldots, k . \\
C_{k+1} \lambda^{-1 / 2} \leq \gamma_{u_{k+1}}^{-1}(0)-\mathfrak{l}_{k+1} \leq C_{k+1}^{\prime} \lambda^{-1 / 2} .
\end{gathered}
$$

Notation. To avoid confusion, we write $u_{\gamma}=\partial_{\gamma} u$ and reserve $u^{\prime}=u_{s}$ for $\partial_{s} u$.

$\gamma_{u_{i}}$ will also be used to denote $\gamma_{u_{i}} \times$ id $:\left(\mathfrak{l}_{i}, \mathfrak{l}_{i+1}\right) \times S^{1} \rightarrow \mathbb{R} \times S^{1}$.

Proof. We will focus on the case of $i=0$, since the cases with other $i$ 's are similar. From the definition, $\gamma_{u_{0}}(s)$ satisfies

where $h_{u_{0}}: \mathbb{R} \rightarrow \mathbb{R}$ is defined as:

$$
\frac{d}{d s} \gamma_{u_{0}}(s)=h_{u_{0}}\left(\gamma_{u_{0}}(s)\right)
$$

$$
h_{u_{0}}(\gamma):= \begin{cases}-\left\langle\left(u_{0}\right)_{\gamma}(\gamma),\left(\bar{\partial}_{J_{\lambda} X_{\lambda}} u_{0}\right)(\gamma)\right\rangle_{2, t}\left\|\left(u_{0}\right)_{\gamma}(\gamma)\right\|_{2, t}^{-2}+1 & \text { when } \gamma \geq 0 \\ 1 & \text { when } \gamma<0\end{cases}
$$

Our choice of the representatives $u_{i}$ ensures that $h_{u_{i}}$ is continuous. ¿From the decay estimates in Proposition I.5.1.3 and the fact that $y$ is in standard d-b neighborhood, for large $\gamma$ we have

$$
\left(\bar{\partial}_{J_{X_{\lambda}}}\right) u_{0}(\gamma)=\delta_{\lambda} \mathcal{V}\left(u_{0}(\gamma)\right)=T_{y, u_{0}(\gamma)}\left(\lambda C_{y}^{\prime} \mathbf{e}_{y}\right)+O\left(\lambda \gamma^{-1}\right)+O\left(\lambda^{2}\right) .
$$

On the other hand, $\left(u_{0}\right)_{\gamma}(\gamma)$ approaches the direction $-\mathbf{e}_{y}$ for large $\gamma$; therefore, there are $\lambda$-independent positive constants $A, A^{\prime}$, such that

$$
A^{\prime} \lambda \gamma^{2} \geq h_{u_{0}}(\gamma) \geq A \lambda \gamma^{2} \quad \text { for } \gamma \gg 1 \text {. }
$$

We see that the inverse function of $\gamma_{u_{0}}(s)$, given by integration

$$
\int_{\gamma_{u_{0}}}^{\infty} \frac{d \gamma}{h_{u_{0}}(\gamma)}=\int_{s\left(\gamma_{u_{0}}\right)}^{0} d s^{\prime}
$$

is well defined where $\gamma_{u_{0}}$ is large. On the other hand, $h_{u_{0}}$ is always positive and goes to 1 when $\gamma_{u_{0}}$ becomes negative; we see that $\gamma_{u_{0}}(s)$ defines a homeomorphism from $\mathbb{R}_{-}$to $\mathbb{R}$.

2.2.2. Definition. The pregluing associated with the gluing data $\chi$ above is $\left(\lambda, w_{\chi}\right) \in \mathcal{B}_{P}^{\Lambda}(\mathbf{x}, \mathbf{z})$, where

$$
w_{\chi}:=e_{R_{-}, R_{+}}\left(0, \underline{w}_{\chi} ; \lambda, 0\right),
$$

$e_{R_{-}, R_{+}}$are defined in I.(62), and

$$
R_{-}=\gamma_{u_{0}}^{-1}\left(-C \lambda^{-1 / 2}\right) ; \quad R_{+}=\gamma_{u_{k+1}}^{-1}\left(C^{\prime} \lambda^{-1 / 2}\right)
$$

for fixed positive constants $C, C^{\prime}$. 
Remark. In general, more complicated pregluing constructions are needed if Definition I.5.3.1 (2b) is not assumed.

The following estimates for $R_{ \pm}$in terms of $\lambda$ will be very useful.

2.2.3. Lemma. $C_{ \pm}^{\prime} \lambda^{-1 / 2} \leq R_{ \pm} \leq C_{ \pm} \lambda^{-1 / 2}$ for some $\lambda$-independent positive constants $C_{ \pm}, C_{ \pm}^{\prime}$.

Proof. We shall only demonstrate the inequalities about $R_{-}$, since those for $R_{+}$are similar.

Choose a large enough $\gamma_{0}$ such that when $s \geq \gamma_{0}$, the decay estimate in Proposition I.5.1.3 for $u_{0}(s)$ and $u_{0}^{\prime}(s)$ holds, and

$$
\left\|\delta_{\lambda} \mathcal{V}\left(u_{0}(s)\right)-T_{y, u_{0}(s)}\left(\lambda \mathbf{e}_{y}\right)\right\|_{2, t} \leq C_{\gamma_{0}} \lambda\left\|u_{0}(s)\right\|_{2, t} .
$$

This implies that when $\gamma \geq \gamma_{0} \lambda^{-1 / 2}, A^{\prime} \lambda \gamma^{2} \geq h_{u_{0}}(\gamma) \geq A \lambda \gamma^{2} \gg 1$ for some $\lambda$-independent constants $A, A^{\prime}$. Thus

$$
C_{2} \lambda^{-1 / 2} \leq-\gamma_{u_{0}}^{-1}\left(\gamma_{0} \lambda^{-1 / 2}\right) \leq \int_{\gamma_{0} \lambda^{-1 / 2}}^{\infty} \frac{d \gamma}{A \lambda \gamma^{2}}=C_{1} \lambda^{-1 / 2}
$$

On the other hand, $\frac{d \gamma_{u_{0}}(s)}{d s}=h_{u_{0}}\left(\gamma_{u_{0}}(s)\right) \geq 1$ always, so

$$
\gamma_{u_{0}}^{-1}\left(\gamma_{0} \lambda^{-1 / 2}\right)-\gamma_{u_{0}}^{-1}\left(-C \lambda^{-1 / 2}\right) \leq C_{2} \lambda^{-1 / 2}
$$

Combining the above two inequalities, we get the claimed inequality for $R_{-}$.

2.3. The weighted norms. Define the weight function $\sigma_{\chi}: \mathbb{R} \rightarrow \mathbb{R}^{+}$by

$$
\sigma_{\chi}(s):= \begin{cases}\left\|w_{\chi}^{\prime}(s)\right\|_{2, t}^{-1} & \text { when } \gamma_{u_{0}}^{-1}(0) \leq s \leq \gamma_{u_{k+1}}^{-1}(0) \\ \left\|w_{\chi}^{\prime}\left(\gamma_{u_{0}}^{-1}(0)\right)\right\|_{2, t}^{-1} & \text { when } s \leq \gamma_{u_{0}}^{-1}(0) \\ \left\|w_{\chi}^{\prime}\left(\gamma_{u_{k+1}}^{-1}(0)\right)\right\|_{2, t}^{-1} & \text { when } s \geq \gamma_{u_{k+1}}^{-1}(0)\end{cases}
$$

Let $\xi \in \Gamma\left(w_{\chi}^{*} K\right)$, define its "longitudinal" component as

$$
\xi_{L}(s):=\beta\left(s-\gamma_{u_{0}}^{-1}(0)\right) \beta\left(\gamma_{u_{k+1}}^{-1}(0)-s\right) \sigma_{\chi}(s)^{2}\left\langle w_{\chi}^{\prime}(s), \xi(s)\right\rangle_{2, t} w_{\chi}^{\prime}(s),
$$

where $\beta: \mathbb{R} \rightarrow[0,1]$ is the smooth cutoff function supported on $\mathbb{R}^{+}$such that $\beta(s)=1 \forall s \geq 1$ (cf. I.3.2.3).

The norms for the domain and range of $E_{w_{\chi}}$ are defined as follows.

2.3.1. Definition. For $\xi \in \Gamma\left(w_{\chi}^{*} K\right)$,

$$
\begin{aligned}
\|\xi\|_{L_{\chi}} & :=\left\|\sigma_{\chi}^{1 / 2} \xi\right\|_{p}+\left\|\sigma_{\chi} \xi_{L}\right\|_{p} \\
\|\xi\|_{W_{\chi}} & :=\left\|\sigma_{\chi}^{1 / 2} \xi\right\|_{p, 1}+\left\|\sigma_{\chi} \xi_{L}^{\prime}\right\|_{p} .
\end{aligned}
$$

As usual, we also use $W_{\chi}, L_{\chi}$ to denote the Banach spaces which are $C^{\infty}$-completion with respect to these norms.

We shall extend the norm $W_{\chi}$ to a norm on $T_{\left(\lambda, w_{\chi}\right)} B_{P}^{\Lambda}(\mathbf{x}, \mathbf{z})=\mathbb{R} \oplus W_{\chi}$ in a way such that $\hat{E}_{\left(\lambda, w_{\chi}\right)}$ is uniformly bounded (cf. Lemma 2.5.1). 
2.3.2. Definition. Define the following norm on $\hat{W}_{\chi}:=\mathbb{R} \oplus W_{\chi}$ (denoted by the same notation):

$$
\|(\alpha, \xi)\|_{\hat{W}_{\chi}}:=\|\xi\|_{W_{\chi}}+\lambda^{-1 /(2 p)-1}|\alpha| .
$$

2.4. The error estimate. The main goal of this subsection is to obtain the following estimate.

2.4.1. Proposition. In the notation of Sections 2.2 and 2.3,

$$
\left\|\bar{\partial}_{J_{\lambda} X_{\lambda}} w_{\chi}\right\|_{L_{\chi}} \leq C \lambda^{1 / 2-1 /(2 p)} \text {. }
$$

Proof. By direct computation, $\bar{\partial}_{J_{\lambda} X_{\lambda}} w_{\chi}$ is supported on $\left(-R_{-}-1, R_{+}+\right.$ 1) $\times S^{1}$, on which it is given by

$$
T_{\underline{w}_{\chi}, w_{\chi}}\left(\tilde{\Pi}_{\underline{w}_{\chi}^{\prime}}^{\perp} \delta_{\lambda} \mathcal{V}\left(\underline{w}_{\chi}\right)\right)+r_{\lambda}(x, z), \quad \text { where }
$$

- $T_{\underline{w}_{\chi}, w_{\chi}}$ is as in Notation I.5.2.6;

- $r_{\lambda}(x, z)$ is a "remainder term" supported on

$$
\left(-R_{-}-1, R_{+}+1\right) \backslash\left(-R_{-}, R_{+}\right) \times S^{1}
$$

which consists of terms involving $\beta\left(-R_{-}-s\right) \bar{x}_{\lambda}^{0, \underline{w}_{\chi}}, \beta\left(s-R_{+}\right) \bar{z}_{\lambda}^{0, \underline{w}} \chi$ and their derivatives (cf. I.(62) for notation);

- letting $\Pi_{\underline{w}_{\chi}^{\prime}(s)}^{\perp}$ denote the $L_{t}^{2}$-orthogonal projection to the orthogonal complement of $\mathbb{R} w_{\chi}^{\prime}(s)$,

$$
\tilde{\Pi}_{\underline{w}_{\chi}^{\prime}(s)}^{\perp}= \begin{cases}\Pi_{\underline{w}_{\chi}^{\prime}(s)}^{\perp} & \text { for } s \in\left[\gamma_{u_{0}}^{-1}(0), \gamma_{u_{k+1}}^{-1}(0)\right] ; \\ \operatorname{Id} & \text { otherwise. }\end{cases}
$$

To estimate the terms in (18), note:

2.4.2. Lemma. When $-R_{-} \leq s \leq R_{+}$, there is a constant $C$ independent of $\lambda$ and $s$, such that

$$
\left\|\sigma_{\chi}(s) \tilde{\Pi}_{w_{\chi}^{\prime}}^{\perp} \delta_{\lambda} \mathcal{V}\left(w_{\chi}(s)\right)\right\|_{\infty, t} \leq C \lambda^{1 / 2} \quad \forall \text { sufficiently small } \lambda .
$$

Combining this lemma with Lemma 2.2.3, one may bound the contribution to $\left\|\bar{\partial}_{J_{\lambda} X_{\lambda}} w_{\chi}\right\|_{L_{\chi}}$ from the first term in (18) by $C_{1} \lambda^{1 / 2-1 /(2 p)}$.

The contribution from the second term can be bounded by $C \lambda$, using the $C^{2}$ bound on $J$ and $X$, and the following estimates:

$$
\begin{aligned}
& \sum_{k=0}^{1} \sup _{s \in\left[-R_{-}-1,-R_{-}\right]}\left\|\partial_{s}^{k} \bar{x}_{\lambda}^{0, \underline{w}_{\chi}}(s)\right\|_{2,1, t} \\
& \quad \leq C_{2} \zeta_{\lambda}^{x}+C_{3} \sum_{k=0}^{1} \sup _{s \in\left[-R_{-}-1,-R_{-}\right]}\left\|\partial_{s}^{k} \mu(s)\right\|_{2,1, t}
\end{aligned}
$$




$$
\begin{aligned}
& \leq C_{2}^{\prime} \lambda+C_{3}^{\prime} \mathrm{e}^{-C_{4} \lambda^{1 / 2}} \\
& \leq C_{x} \lambda,
\end{aligned}
$$

where $\mu(s), \zeta_{\lambda}^{x}$ are defined by $\exp \left(\underline{w}_{\chi}(s), \mu(s)\right)=x_{0}, \exp \left(x_{0}, x_{\lambda}\right)=\zeta_{\lambda}^{x}$, and the second inequality follows from the exponential decay of $\underline{w}_{\chi}$ to $x_{0}$, and the estimate for $R_{-}$in Lemma 2.2.3. Similarly,

$$
\sum_{k=0}^{1} \sup _{s \in\left[R_{+}, R_{+}+1\right]}\left\|\partial_{s}^{k} \bar{z}_{\lambda}^{0, \underline{w}_{\chi}}(s)\right\|_{2,1, t} \leq C_{z} \lambda .
$$

These together imply the proposition.

Proof of Lemma 2.4.2. By Sobolev embedding, it suffices to estimate the $L_{1, t}^{2}$ norm. Again we will estimate only the $s \leq 0$ part, since the other parts are entirely similar. Let $\gamma_{0}$ be as in Lemma 2.2.3. Consider the following two cases separately. Case 1: $-R_{-} \leq s \leq \gamma_{u_{0}}^{-1}\left(\gamma_{0}\right)$; Case $2: \gamma_{u_{0}}^{-1}\left(\gamma_{0}\right) \leq s \leq 0$. Case 1: In this region, $\| \tilde{\Pi}_{w_{\chi}^{\prime}}^{\perp}\left(\delta_{\lambda} \mathcal{V}\left(w_{\chi}(s)\right) \|_{2,1, t} \leq C \lambda\right.$. On the other hand, on this region, $\sigma_{\chi} \leq C$; in sum, we have $\| \sigma_{\chi} \tilde{\Pi}_{w_{\chi}^{\prime}}^{\perp}\left(\delta_{\lambda} \mathcal{V}\left(w_{\chi}(s)\right) \|_{2,1, t} \leq C_{3} \lambda\right.$.

Case 2: In this region, the fact that $y$ is in a standard d-b neighborhood plus the decay estimates in Proposition I.5.1.3 imply that for small enough $\lambda$,

$$
\begin{aligned}
\| \Pi_{\left(u_{0}\right)_{\gamma}}^{\perp}\left(\delta_{\lambda} \mathcal{V}\left(u_{0}\left(\gamma_{u_{0}}\right)\right) \|_{2, t}\right. & \leq \lambda C_{0}\left(\left\|\Pi_{\left(u_{0}\right)_{\gamma}}^{\perp} T_{y, u_{0}\left(\gamma_{u_{0}}\right)} \mathbf{e}_{y}\right\|_{2, t}+\left\|\mu\left(\gamma_{u_{0}}\right)\right\|_{2, t}\right) \\
& \leq \lambda\left(\left(1-\left(1+C_{1}^{2}\left\|b\left(\gamma_{u_{0}}\right)\right\|_{2, t}^{2}\right)^{-1}\right)^{1 / 2}+C^{\prime \prime} \gamma_{u_{0}}^{-1}\right. \\
& \leq \lambda\left(C_{1}\left\|b\left(\gamma_{u_{0}}\right)\right\|_{2, t}+C^{\prime \prime} \gamma_{u_{0}}^{-1}\right) \\
& \leq C_{4} \lambda \gamma_{u_{0}}^{-1},
\end{aligned}
$$

where $\mu, b$ are defined by $\exp (y, \mu(\gamma))=u_{0}(\gamma), b(\gamma)=\Pi_{\mathbf{e}_{y}} \mu(\gamma)$, as in Section I.5. Meanwhile,

$$
\begin{aligned}
\left\|\partial_{t}\left[\Pi_{\left(u_{0}\right)_{\gamma}}^{\perp} \delta_{\lambda} \mathcal{V}\left(u_{0}\left(\gamma_{u_{0}}\right)\right)\right]\right\|_{2, t} \\
\quad \leq\left\|\partial_{t}\left[\delta_{\lambda} \mathcal{V}\left(u_{0}\left(\gamma_{u_{0}}\right)\right)\right]\right\|_{2, t}+\left\|\partial_{t}\left(\Pi_{u_{\gamma_{u_{0}}}}\right)\left(\delta_{\lambda} \mathcal{V}\left(u_{0}\left(\gamma_{u_{0}}\right)\right)\right)\right\|_{2, t} \\
\quad \leq \lambda C_{5}\left(\left\|\partial_{t}\left(u_{0}\left(\gamma_{u_{0}}\right)\right)\right\|_{2, t}+\left\|\sigma_{u} \partial_{t}\left(u_{0}\right)_{\gamma}\left(\gamma_{u_{0}}\right)\right\|_{2, t}\right) \\
\quad \leq C_{6} \lambda \gamma_{u_{0}}^{-1} .
\end{aligned}
$$

On the other hand, we have from direct computation:

$$
\begin{aligned}
\sigma_{\chi}^{-1}(s) & =\left\|w_{\chi}^{\prime}(s)\right\|_{2, t} \\
& =\Pi_{\left(u_{0}\right)_{\gamma}\left(\gamma_{u_{0}}(s)\right)}\left(\delta_{\lambda} \mathcal{V}\left(u_{0}\left(\gamma_{u_{0}}(s)\right)\right)\right)+\left\|\left(u_{0}\right)_{\gamma}\left(\gamma_{u_{0}}(s)\right)\right\|_{2, t}
\end{aligned}
$$

when $\gamma_{u_{0}}^{-1}(0) \leq s \leq 0$. In particular,

$$
C^{\prime}\left(\lambda+\gamma_{u_{0}}(s)^{-2}\right) \geq\left\|w_{\chi}^{\prime}(s)\right\|_{2, t} \geq C_{7}\left(\lambda+\gamma_{u_{0}}(s)^{-2}\right)
$$


when $0 \geq s \geq \gamma_{u_{0}}^{-1}\left(\gamma_{0}\right)$.

In sum, in Case 2

$$
\sigma_{\chi}(s)\left\|\Pi_{w_{\chi}^{\prime}}^{\perp} \delta_{\lambda} \mathcal{V}\left(w_{\chi}(s)\right)\right\|_{2,1, t} \leq \frac{C_{8} \lambda \gamma_{u_{0}}(s)^{-1}}{\lambda+\gamma_{u_{0}}(s)^{-2}} \leq C_{9} \lambda^{1 / 2} .
$$

The last step above is obtained by a simple estimate of the critical value of the rational function. Combining the two cases, we have proved the lemma.

2.5. Bounding linear and nonlinear terms. In the previous subsection, we obtained the estimate for the 0-th-order term of the expansion (1). We estimate the linear and nonlinear terms in this subsection. In our context, this means bounding $\hat{E}_{\left(\lambda, w_{\chi}\right)}$ and $\hat{n}_{\left(\lambda, w_{\chi}\right)}$. These are done, respectively, in Lemmas 2.5.1 and 2.5.2 below.

2.5.1. Lemma. With respect to the norms $\hat{W}_{\chi}, L_{\chi}$ of Section 2.3 , the deformation operator $\hat{E}_{\left(\lambda, w_{\chi}\right)}$ is bounded uniformly in $\lambda$.

Proof. The uniform boundedness of $E_{w_{\chi}}$ follows from simple adaptation of I.5.2.3. We therefore just have to estimate the $L_{\chi}$ norm of

$$
\hat{E}_{\left(\lambda, w_{\chi}\right)}(1,0)=Y_{\left(\lambda, w_{\chi}\right)},
$$

where $Y_{\left(\lambda, w_{\chi}\right)}$ is as in I.(63). By the properties of $Y_{\left(\lambda, w_{\chi}\right)}$ listed following I.(63), $Y_{\left(\lambda, w_{\chi}\right)}$ is supported on $\left(-R_{-}-1, R_{+}+1\right) \times S^{1}$, over which it has a $\lambda$-independent $C_{\epsilon}^{\infty}$ bound. Also, from (19), we have $\sigma_{\chi} \leq C \lambda^{-1}$. These, together with Lemma 2.2.3, imply

$$
\left\|\hat{E}_{\left(\lambda, w_{\chi}\right)}(1,0)\right\|_{L_{\chi}} \leq C^{\prime} \lambda^{-1-1 /(2 p)}=C^{\prime}\|(1,0)\|_{\hat{W}_{\chi}} .
$$

for a $\lambda$-independent positive constant $C^{\prime}$.

Given $(\alpha, \xi) \in T_{\left(\lambda, w_{\chi}\right)} \mathcal{B}_{P}^{\Lambda}(\mathbf{x}, \mathbf{z})$, let $\hat{n}_{\left(\lambda, w_{\chi}\right)}(\alpha, \xi)$ be

$$
T_{w_{\chi}, e\left(\lambda, w_{\chi} ; \alpha, \xi\right)}^{-1} \bar{\partial}_{J^{\Lambda}, X^{\Lambda}}\left(\lambda+\alpha, e\left(\lambda, w_{\chi} ; \alpha, \xi\right)\right)-\bar{\partial}_{J_{\lambda} X_{\lambda}} w_{\chi}-\hat{E}_{\left(\lambda, w_{\chi}\right)}(\alpha, \xi) .
$$

2.5.2. Lemma. There is a $\lambda$-independent constant $C_{n}$ such that for any $\hat{\xi}=(\alpha, \xi), \hat{\eta}=\left(\alpha^{\prime}, \eta\right) \in \hat{W}_{\chi}$,

$$
\left\|\hat{n}_{\left(\lambda, w_{\chi}\right)}(\hat{\xi})-\hat{n}_{\left(\lambda, w_{\chi}\right)}(\hat{\eta})\right\|_{L_{\chi}} \leq C_{n}\left(\|\hat{\xi}\|_{\hat{W}_{\chi}}+\|\hat{\eta}\|_{\hat{W}_{\chi}}\right)\|\hat{\xi}-\hat{\eta}\|_{\hat{W}_{\chi}} .
$$

Proof. These follow from direct computations, via the $C_{\epsilon}^{\infty}$-bounds of $J, X$. First, observe the pointwise estimate

$$
\begin{aligned}
& \left|\hat{n}_{\left(\lambda, w_{\chi}\right)}(\hat{\xi})-\hat{n}_{\left(\lambda, w_{\chi}\right)}(\hat{\eta})\right| \\
& \quad \leq C_{1}(|\xi|+|\eta|)(|\xi-\eta|+|\nabla(\xi-\eta)|)+C_{2}(|\nabla \xi|+|\nabla \eta|)|\xi-\eta| \\
& \quad+\left(|\alpha|+\left|\alpha^{\prime}\right|\right)\left(\left|\alpha-\alpha^{\prime}\right|\right)\left|Z_{\lambda \lambda}\right|+\left(\left(|\alpha|+\left|\alpha^{\prime}\right|\right)|\xi-\eta|+\left|\alpha-\alpha^{\prime}\right|(|\xi|+|\eta|)\right)\left|Z_{\lambda w}\right|,
\end{aligned}
$$


where $Z_{\lambda \lambda}, Z_{\lambda w}$ are both supported on $\left(-R_{-}-1, R_{+}+1\right) \times S^{1}$, over which they are $\partial_{\lambda}^{2} \check{\theta}_{X_{\lambda}}\left(w_{\chi}\right), \partial_{\lambda} \nabla \check{\theta}_{X_{\lambda}}\left(w_{\chi}\right) / 2$, respectively, up to ignorable terms. Estimating similarly to the proof of Lemma 2.5.1, we have

$$
\left\|\sigma_{\chi} Z_{\lambda \lambda}\right\|_{p, 1}+\left\|\sigma_{\chi} Z_{\lambda w}\right\|_{p, 1} \leq C^{\prime} \lambda^{-1-1 /(2 p)} .
$$

Thus

$$
\begin{aligned}
\left\|\hat{n}_{\left(\lambda, w_{\chi}\right)}(\hat{\xi})-\hat{n}_{\left(\lambda, w_{\chi}\right)}(\hat{\eta})\right\|_{L_{\chi}} \leq\left\|\sigma_{\chi}\left(\hat{n}_{\left(\lambda, w_{\chi}\right)}(\hat{\xi})-\hat{n}_{\left(\lambda, w_{\chi}\right)}(\hat{\eta})\right)\right\|_{p, 1} \\
\leq C_{1}\left(\left\|\sigma_{\chi}^{1 / 2} \xi\right\|_{\infty}+\left\|\sigma_{\chi}^{1 / 2} \eta\right\|_{\infty}\right)\|\xi-\eta\|_{W_{\chi}} \\
\quad+C_{2}\left(\|\xi\|_{W_{\chi}}+\|\eta\|_{W_{\chi}}\right)\left\|\sigma_{\chi}^{1 / 2}(\xi-\eta)\right\|_{\infty} \\
\quad+C^{\prime} \lambda^{-1-1 /(2 p)}\left(|\alpha|+\left|\alpha^{\prime}\right|\right)\left(\left|\alpha-\alpha^{\prime}\right|\right) \\
\quad+C^{\prime} \lambda^{-1-1 /(2 p)}\left(\left(|\alpha|+\left|\alpha^{\prime}\right|\right)\|\xi-\eta\|_{\infty}+\left|\alpha-\alpha^{\prime}\right|\left(\|\xi\|_{\infty}+\|\eta\|_{\infty}\right)\right) \\
\leq C_{n}\left(\|\hat{\xi}\|_{\hat{W}_{\chi}}+\|\hat{\eta}\|_{\hat{W}_{\chi}}\right)\|\hat{\xi}-\hat{\eta}\|_{\hat{W}_{\chi}},
\end{aligned}
$$

using a Sobolev inequality to bound the $L^{\infty}$-norm by $L_{1}^{p}$-norm.

\section{Gluing at Deaths II: the Kuranishi structure}

The purpose of this section is to introduce a K-model for the operator $E_{w_{\chi}}$. By stabilization, this also yields a K-model for $\hat{E}_{\left(\lambda, w_{\chi}\right)}$. The main result is summarized in Proposition 3.1.3.

3.1. The generalized kernel and generalized cokernel. Given $y \in \mathcal{P}$, we denote by $\bar{y}$ the constant flow $\bar{y}(s)=y \forall s$.

We first partition the domain $\Theta=\mathbb{R} \times S^{1}$ into several regions, over which $w_{\chi}$ approximates either one of $u_{i}$ or $\bar{y}$.

For a subdomain $\Theta^{\prime} \subset \Theta$ and some norm $L$, we denote by $\|\xi\|_{L\left(\Theta^{\prime}\right)}:=$ $\left\|\left.\xi\right|_{\Theta^{\prime}}\right\|_{L}$

3.1.1. Definition (Partioning $\Theta$ ). Fix a small positive number $\epsilon>\lambda$. For $i=0, \ldots, k+1$, let

$$
\mathfrak{r}_{i}:=\left(2 C_{u_{i}} \epsilon\right)^{1 / 2}\left(\lambda C_{y}^{\prime}\right)^{-1 / 2},
$$

where $C_{u_{i}}$ is the constant in the bound $\left\|u_{i}^{\prime}(s)\right\|_{2} \geq C_{u_{i}} / s^{2}$ (cf. I.5.1.3). For $j=1, \ldots, k+1$, define

$$
\begin{aligned}
\Theta_{y j}:= & {\left[\mathfrak{s}_{j-}, \mathfrak{s}_{j+}\right] \times S^{1}, \quad \text { where } } \\
& \mathfrak{s}_{j-}:=\gamma_{u_{j-1}}^{-1}\left(\mathfrak{r}_{j-1}\right), \quad \mathfrak{s}_{j+}=\gamma_{u_{j}}^{-1}\left(-\mathfrak{r}_{j}\right) .
\end{aligned}
$$

Let $\Theta_{u_{i}}$ denote the $(i+1)$ th component of $\Theta \backslash \bigcup_{j} \Theta_{y j}$, and let $\Theta_{y j}^{\prime}=$ $\left(\gamma_{u_{j-1}}^{-1}\left(\mathfrak{r}_{j-1}-1\right), \gamma_{u_{j}}^{-1}\left(-\mathfrak{r}_{j}+1\right)\right) \times S^{1} \supset \Theta_{y j}$. 
Notice that the "length" of the region $\Theta_{y j}, \mathfrak{s}_{j+}-\mathfrak{s}_{j-}$, is bounded as

$$
C_{1}(\epsilon \lambda)^{-1 / 2} \leq \mathfrak{s}_{j+}-\mathfrak{s}_{j-} \leq C_{2}(\epsilon \lambda)^{-1 / 2} .
$$

These inequalities follow from the arguments leading to (16), using, respectively, inequalities of the type of the left and the right inequalities in (14). The length of $\Theta_{y j}^{\prime}$ satisfies similar bounds, with the constants $C_{1}, C_{2}$ above replaced by different constants $C_{1}^{\prime}, C_{2}^{\prime}$.

3.1.2. Definition (Bases for generalized kernel/cokernel). For $i=$ $1, \ldots, k+1$, let

$$
\mathfrak{e}_{u_{i}}:=\gamma_{u_{i}}^{*} u_{i}^{\prime} \in W_{\chi} .
$$

For $j=1, \ldots, k+1$, define the following elements in $L_{\chi}$ :

$$
\mathfrak{f}_{j}:=C_{j}|\lambda|^{1+1 /(2 p)} \vartheta_{\Theta_{y j}} w_{\chi}^{\prime}\left\|w_{\chi}\right\|_{2, t}^{-1},
$$

where $\vartheta_{\Theta_{y j}}$ is a characteristic function supported on $\Theta_{y j}$ and $C_{j}$ are constants chosen such that $\left\|\mathfrak{f}_{j}\right\|_{L_{\chi}}=1$.

$$
\begin{aligned}
\text { Let } & \\
K_{\chi} & :=\operatorname{Span}\left\{\mathfrak{e}_{u_{i}}\right\}_{i \in\{0, \ldots, k+1\}} \subset W_{\chi} ; \\
\hat{K}_{\chi} & :=\operatorname{Span}\left\{(1,0),\left(0, \mathfrak{e}_{u_{i}}\right)\right\}_{i \in\{0, \ldots, k+1\}} \subset \hat{W}_{\chi} ; \\
C_{\chi} & :=\operatorname{Span}\left\{\mathfrak{f}_{j}\right\}, \quad \text { and } \\
W_{\chi}^{\prime} & :=\left\{\xi \mid\left\langle\left(\gamma_{u_{i}}^{-1}\right)^{*} \xi(0), \eta(0)\right\rangle_{2, t}=0 \forall \eta \in \operatorname{ker} E_{u_{i}} \forall i \in\{0,1, \ldots, k+1\}\right\} \\
& \subset W_{\chi} .
\end{aligned}
$$

(Note that linearly independent elements in ker $E_{u_{i}}$ restrict linearly independently to the circle $s=0$, since they satisfy a homogeneous first-order differential equation.)

A quick computation shows that the $W_{\chi}$-norm on $K_{\chi}$ and the $L_{\chi}$-norm on $C_{\chi}$ are commensurate with the standard norm on Euclidean spaces with respect to the bases given above.

These are, respectively, fibers of Banach spaces bundles over the space of gluing parameters $\Xi(\mathbb{S}), K^{\Xi}, \hat{K}^{\Xi}, C^{\Xi}, W^{\prime} \Xi$, and $\hat{W}^{\prime} \Xi$.

Obviously, $W_{\chi}=K_{\chi} \oplus W_{\chi}^{\prime}$ and $\hat{W}_{\chi}=\hat{K}_{\chi} \oplus W_{\chi}^{\prime}$. Let $\tilde{W}_{\chi}:=\mathbb{R}^{k+1} \oplus W_{\chi}^{\prime}$, with the standard metric on $\mathbb{R}^{k+1}$. (As usual, we denote the norm on it by the same notation.) Let

$$
\tilde{E}_{\chi}: \tilde{W}_{\chi} \rightarrow L_{\chi}, \quad \tilde{E}_{\chi}\left(\iota_{1}, \ldots, \iota_{k+1}, \xi\right):=E_{w_{\chi}} \xi+\sum_{j=1}^{k+1} \iota_{j} \mathfrak{f}_{j} .
$$

A quick computation using (21) shows that this is a bounded operator. The rest of this section is devoted to proving the following. 
3.1.3. Proposition. For sufficiently small $\lambda_{0}$, the triples $K^{\Xi}, C^{\Xi}, W^{\prime} \Xi$, and $\hat{K}^{\Xi}, C^{\Xi}, W^{\prime} \Xi$ are, respectively, K-models for the families of operators $\left\{E_{w_{\chi}}\right\}_{\chi \in \Xi(\mathbb{S})}$ and $\left\{\hat{E}_{\left(\lambda, w_{\chi}\right)}\right\}_{\chi \in \Xi(\mathbb{S})}$.

In particular, there is an inverse $\tilde{G}_{\chi}: L_{\chi} \rightarrow \tilde{W}_{\chi}$ of $\tilde{E}_{\chi}$, which is bounded uniformly in $\lambda$.

We shall concentrate on proving the existence of a uniformly bounded $\tilde{G}_{\chi}$, since the rest of the assertions follow in a straightforward manner. The proof follows the "proof by contradiction" framework outlined in Section 1.2.3; since $\tilde{E}_{\chi}$ is Fredholm with ind $\tilde{E}_{\chi}=0$, it suffices to show that there exists a $\lambda$-independent constant $C$, such that $\|\tilde{\xi}\|_{L_{\chi}} \leq C\left\|\tilde{E}_{\chi} \tilde{\xi}\right\|_{\tilde{W}_{\chi}} \forall \tilde{\xi} \in \tilde{W}_{\chi}$.

Suppose the contrary that there exists a sequence

$$
\begin{gathered}
\left\{\tilde{\xi}_{\lambda}=\left(\iota_{1, \lambda}, \ldots, \iota_{k+1, \lambda}, \xi_{\lambda}\right) \in \tilde{W}_{\chi}\right\}, \quad \text { with }\left\|\tilde{\xi}_{\lambda}\right\|_{\hat{W}_{\chi}}=1 \text { and } \\
\left\|\hat{E}_{\left(\lambda, w_{\chi}\right)}\left(\tilde{\xi}_{\lambda}\right)\right\|_{L_{\chi}}=: \varepsilon_{E}(\lambda) \rightarrow 0 \quad \text { where } \lambda \rightarrow 0 .
\end{gathered}
$$

We shall estimate $\tilde{\xi}_{\lambda}$ in terms of $\varepsilon_{E}(\lambda)$ over the various domains introduced in Definition 3.1.1 to obtain a contradiction.

3.2. Estimates over $\Theta_{u_{i}}$. Given a diffeomorphism $\gamma: I \rightarrow \mathbb{R}$, let

$$
\mathcal{T}_{w, \underline{w}}^{\gamma}:=\left(\gamma^{-1}\right)^{*} T_{w, \underline{w}}: \Gamma\left(\Theta, w^{*} K\right) \rightarrow \Gamma\left(\gamma(\Theta),\left(\gamma^{-1}\right)^{*} \underline{w}^{*} K\right) .
$$

By construction, for $\xi \in \Gamma\left(w_{\chi}^{*} K\right), \mathcal{T}_{w_{\chi}, \underline{w}_{\chi}}^{\gamma u_{i}} \xi \in \Gamma\left(u_{i}^{*} K\right)$.

Since the discussion in this subsection holds for all $i$, we shall often drop the index $i$. For instance, $u=u_{i}$ for some $i$.

In this subsection, the "transversal" or "longitudinal components" shall refer to the respective components of elements in $\Gamma\left(u^{*} K\right)$.

3.2.1. Comparing $W_{\chi}, L_{\chi}$-norms and $W_{u}, L_{u}$-norms. According to computation in the proof of Lemma 2.2.1 and the definition of $\Theta_{u}$, in this region $\left|h_{u}\left(\gamma_{u}\right)-1\right| \leq \epsilon$. Thus, $\sigma_{u}$ and $\sigma_{\chi}$ are close in this region, and by direct computation we have the following lemma.

Lemma. Suppose $\xi \in \Gamma\left(w_{\chi}^{*} K\right)$ is supported on $\Theta_{u}$, and let $\epsilon, \lambda$ be as in Definition 3.1.1. Then

$$
\begin{gathered}
(1-2 \epsilon)\|\xi\|_{W_{\chi}} \leq\left\|\mathcal{T}_{w_{\chi}, \underline{w}_{\chi}}^{\gamma_{u}} \xi\right\|_{W_{u}} \leq(1+2 \epsilon)\|\xi\|_{W_{\chi}} \\
(1-2 \epsilon)\|\xi\|_{L_{\chi}} \leq\left\|\mathcal{T}_{w_{\chi}, \underline{w}_{\chi}}^{\gamma_{u}} \xi\right\|_{L_{u}} \leq(1+2 \epsilon)\|\xi\|_{L_{\chi}}
\end{gathered}
$$

and for some constant $C$,

$$
\begin{aligned}
& \left\|\hat{E}_{\left(\lambda, w_{\chi}\right)}(\alpha, \xi)\right\|_{L_{\chi}} \\
& \quad \geq(1+2 \epsilon)^{-1}\left\|E_{u}\left(\mathcal{T}_{w_{\chi}, \underline{w}_{\chi}}^{\gamma_{u}} \xi\right)+\alpha \mathcal{T}_{w_{\chi}, \underline{w}_{\chi}}^{\gamma_{u}} Y_{\left(\lambda, w_{\chi}\right)}\right\|_{L_{u}}-(C \lambda+\epsilon)\|\xi\|_{W_{\chi}} .
\end{aligned}
$$


Remark. The fact that $y$ is in a standard d-b neighborhood (more precisely, the condition Definition I.5.3.1(2c)) is used here. In general, the last term on the right-hand side of the above inequality would be larger.

3.2.2. From $\xi_{\lambda} \in W_{\chi}^{\prime}$ to $\bar{\xi}_{u_{i}, \lambda} \in W_{u_{i}}^{\prime}$. For $i=1, \ldots, k$, and $\xi_{\lambda} \in W_{\chi}^{\prime}$, let

$$
\bar{\xi}_{u_{i}, \lambda}:= \begin{cases}\left(\gamma_{u_{i}}^{-1}\right)^{*} \xi_{\lambda}-\beta_{i}\left(\left(\gamma_{u_{i}} g^{-1}\right)^{*} \xi_{\lambda}\right)_{T} & \\ \left.\left.-\theta_{i+}\left(\left(\gamma_{u_{i}}^{-1}\right)^{*} \xi_{\lambda}\right)_{L}-c_{i+} u_{i}^{\prime}\right)-\theta_{i-}\left(\left(\gamma_{u_{i}}^{-1}\right)^{*} \xi_{\lambda}\right)_{L}-c_{i-} u_{i}^{\prime}\right) & \text { on }\left(-\mathfrak{r}_{i}, \mathfrak{r}_{i}\right) \times S^{1} \\ 0 & \text { outside. }\end{cases}
$$

where

- $\beta_{i}$ is a smooth cutoff function in $s$ supported away from $\left(-\mathfrak{r}_{i}+1, \mathfrak{r}_{i}-1\right)$, being 1 outside $\left(-\mathfrak{r}_{i}, \mathfrak{r}_{i}\right)$.

- $\theta_{i \pm}$ are characteristic functions of $\left(-\infty-\mathfrak{s}_{i}\right)$ and $\left(\mathfrak{s}_{i}, \infty\right)$, respectively.

- $c_{i \pm}$ are constants defined by

$$
\left(\left(\gamma_{u_{i}}^{-1}\right)^{*} \xi_{\lambda}\right)_{L}\left( \pm \mathfrak{r}_{i}\right)=c_{i \pm} u^{\prime}\left( \pm \mathfrak{r}_{i}\right)
$$

For $i=0$ or $k+1$ and similarly defined constants $c_{0}, c_{k+1}$, let

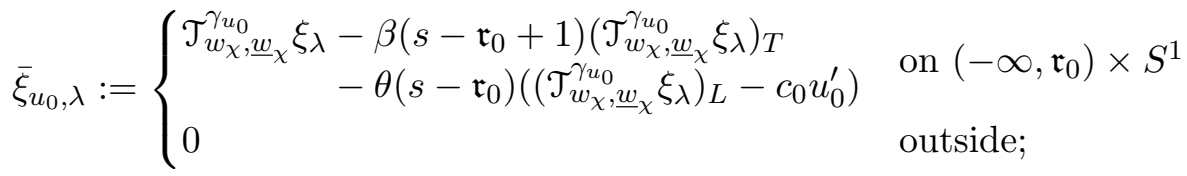

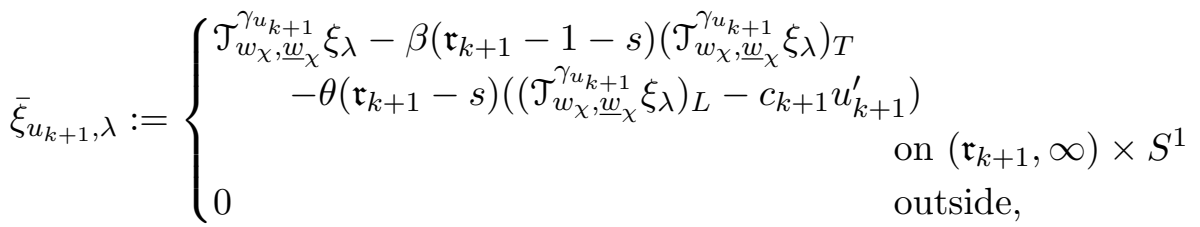

where $\beta$ is the smooth cutoff function as in Part I and Section 1.2.2, $\theta$ is the characteristic function of $\mathbb{R}^{+}$.

Remark. The point of the above definition is to introduce cutoff on $\xi_{\lambda}$, while keeping the extra terms (arising from the cutoff) in $E_{u}\left(\bar{\xi}_{\lambda}\right)$ ignorable. The usual smooth cutoff works for the transversal direction, but not for the longitudinal component, over which the weight function is greater. Instead, we replace the longitudinal component over the cutoff region by a suitable multiple of $u^{\prime}$ determined by the matching condition (24) and make use of the fact that $E_{u}\left(u^{\prime}\right)=0$. 
3.2.3. Estimating $E_{u} \bar{\xi}_{u, \lambda}$. The estimate for all $i$ is similar. Taking $i=0$, for example, a straightforward computation yields:

$$
\begin{aligned}
E_{u_{0}}\left(\bar{\xi}_{u_{0}, \lambda}\right)=(1 & \left.-\beta\left(s-\mathfrak{r}_{0}+1\right)\right) E_{u_{0}}\left(\mathcal{T}_{w_{\chi}, \underline{w}_{\chi}}^{\gamma_{u_{0}}} \xi_{\lambda T}\right) \\
& +\left(1-\theta\left(s-\mathfrak{r}_{0}\right)\right) E_{u_{0}}\left(\mathcal{T}_{w_{\chi}, \underline{w}_{\chi}}^{\gamma_{u_{0}}} \xi_{\lambda L}\left(\mathfrak{r}_{0}\right)\right) \\
& -\beta^{\prime}\left(s-\mathfrak{r}_{0}+1\right) \mathcal{T}_{w_{\chi}, \underline{w}_{\chi}}^{\gamma_{u_{0}}} \xi_{\lambda T} \\
& \left.-\delta\left(s-\mathfrak{r}_{0}\right)\left(\mathcal{T}_{w_{\chi}, \underline{w}_{\chi}}^{\gamma_{u_{0}}} \xi_{\lambda L}-c_{0} u_{0}^{\prime}\right)\right) .
\end{aligned}
$$

The last term above has vanishing $L_{u}$-norm because of the condition (24); by Lemma 3.3.1, the $L_{u}$-norm of the penultimate term can be bounded by $C \varepsilon_{0}(\lambda)$, which goes to 0 as $\lambda \rightarrow 0$. Thus, by the previous lemma, we have for small $\lambda$ that

$$
\begin{aligned}
& \left\|E_{u} \bar{\xi}_{u, \lambda}\right\|_{L_{u}\left(\gamma_{u}\left(\Theta_{u}\right)\right)} \\
\leq & \left\|E_{u}\left(\mathcal{T}_{w_{\chi}, \underline{w}_{\chi}}^{\gamma_{u}} \xi_{\lambda}\right)\right\|_{L_{u}\left(\gamma_{u}\left(\Theta_{u}\right)\right)}+C \varepsilon_{0}(\lambda) \\
\leq & (1+2 \epsilon)\left\|E_{w_{\chi}} \xi_{\lambda}\right\|_{L_{\chi}\left(\Theta_{u}\right)}+\left(C^{\prime} \lambda+\epsilon\right)\left\|\xi_{\lambda}\right\|_{W_{\chi}\left(\Theta_{u}\right)}+C \varepsilon_{0}(\lambda)
\end{aligned}
$$

In the last expression, the first term goes to zero because of (22) and the fact that over $\Theta_{u}, \tilde{E}_{\chi} \tilde{\xi}_{\lambda}=E_{w_{\chi}} \xi_{\lambda}$. The second term is small since $\left\|\xi_{\lambda}\right\|_{W_{\chi}} \leq 1$.

3.2.4. Estimating $\bar{\xi}_{u_{i}, \lambda}$. Since $\bar{\xi}_{u, \lambda} \in W_{u}^{\prime}$, where

$$
W_{u}^{\prime}:=\left\{\xi \mid \xi \in W_{u},\left\langle u^{\prime}(0), \xi(0)\right\rangle_{2, t}=0\right\},
$$

by the right-invertibility of $E_{u},\left\|\bar{\xi}_{u, \lambda}\right\|_{W_{u}} \leq C\left\|E_{u} \bar{\xi}_{u, \lambda}\right\|_{L_{u}} \leq \varepsilon$. In particular,

$$
\left\|\mathcal{T}_{w_{\chi}, \underline{w}_{\chi}}^{\gamma_{u}} \xi_{\lambda}\right\|_{W_{u}\left(\gamma_{u}\left(\Theta_{u}\right)\right)} \leq \varepsilon_{u} \quad \text { when } \lambda \leq \lambda_{0} \text { is sufficiently small, }
$$

where $\varepsilon_{u}$ is of the form

$$
\varepsilon_{u}=C \varepsilon_{0}(\lambda)+C_{2}(\lambda+\epsilon)+2 \varepsilon_{E}(\lambda),
$$

which can be made arbitrarily small by choosing the small constants $\epsilon, \lambda$ appropriately.

3.3. Estimates over $\Theta_{y j}$. The estimates over $\Theta_{y j}$ for different $j$ are similar, so we shall drop the subscript $j$ in the discussion below.

First, note that from the computation of (20) there exist constants $C_{M}, C_{m}$ independent of $\lambda$ such that

$$
C_{m} \lambda^{-1} \leq \sigma_{\chi}(s) \leq C_{M} \lambda^{-1} \quad \text { on } \Theta_{y} .
$$

We may therefore replace (modulo multiplication by a constant) the weights in the $W_{\chi}$ and $L_{\chi}$ norms by $\lambda^{-1}$. 
3.3.1. Estimating the transversal component. In the transversal direction, the estimates are again similar to the standard case: By looking at the limit of $\left(\alpha_{\lambda}, \xi_{\lambda}\right)$, one has:

Lemma (Floer). Let $\left(\alpha_{\lambda}, \xi_{\lambda}\right)$ be as in (22). Then for all sufficiently small $\lambda$,

$$
\left\|\xi_{\lambda}\right\|_{L^{\infty}\left(\Theta_{y}^{\prime}\right)} \leq \varepsilon_{0}(\lambda) \lambda^{1 / 2}
$$

where $\varepsilon_{0}(\lambda)$ is a small positive number, $\lim _{\lambda \rightarrow 0} \varepsilon_{0}(\lambda)=0$.

Proof. Let $\left(s_{\lambda}, t_{\lambda}\right)$ be a maximum of $\left|\xi_{\lambda}\right|$ in $\Theta_{y}^{\prime}$. Consider a slight enlargement of $\Theta_{y}^{\prime}, \Theta_{y}^{\prime \prime} \supset \Theta_{y}^{\prime}$, and let $C>0$ be such that

$$
\left[-C^{-1}(\epsilon \lambda)^{-1 / 2}, C^{-1}(\epsilon \lambda)^{-1 / 2}\right] \times S^{1} \subset \Theta_{y}^{\prime \prime} .
$$

Define

$$
\varsigma_{\lambda}(s, t):=\lambda^{-1 / 2} \tilde{\beta}\left(C(\epsilon \lambda)^{1 / 2} s\right) T_{w_{\chi} \bar{y}} \xi_{\lambda}\left(s+s_{\lambda}, t\right) \quad \text { on } \Theta_{y}^{\prime \prime},
$$

where $\tilde{\beta}$ is a smooth cutoff function supported on $(-1,1)$ which equals 1 on $(-1 / 2,1 / 2)$. By $(22),\left\|\varsigma_{\lambda}\right\|_{p, 1}$ is uniformly bounded and thus by Sobolev embedding $\varsigma_{\lambda}$ converges in $C_{0}$ (taking a subsequence if necessary) to a $\varsigma_{0}$, which satisfies $E_{\bar{y} \varsigma_{0}}=0$. (Note that the term involving $\iota$ dropped out because of the assumption $|\iota| \leq \lambda^{1+1 /(2 p)}$.) Such a $\varsigma_{0}$ must be identically zero cf. pp. 542-543 of [6]; so

$$
\left\|\varsigma_{\lambda}\right\|_{L^{\infty}\left([-1,1] \times S^{1}\right)} \rightarrow 0 \text { as } \lambda \rightarrow 0,
$$

and thus $\left\|\xi_{\lambda}\right\|_{L^{\infty}\left(\Theta_{y}^{\prime}\right)}<\varepsilon_{0}(\lambda) \lambda^{1 / 2}$.

Remark. In fact, one may be more precise about the longitudinal component: by part (a) of Lemma 3.3.3, $\left\|\xi_{\lambda L}\right\|_{L^{\infty}\left(\Theta_{y}^{\prime}\right)} \leq C \lambda^{1 / 2+1 /(2 p)} \epsilon^{1 /(2 p)-1 / 2}$.

Lemma 3.3.1 tells us that $\left\|E_{w_{\chi}}\left(\beta_{y} \xi_{\lambda}\right)_{T}\right\|_{L_{\chi}} \rightarrow 0$, where $\beta_{y}$ is a smooth cutoff function supported on $\Theta_{y}^{\prime \prime}$ with value 1 on $\Theta_{y}^{\prime}$, since contribution from the extra term due to the cutoff function goes to zero. Thus since $E_{w_{\chi}}$ is right-invertible (being close to a conjugation of $E_{\bar{y}}$ ) on the transversal subspace,

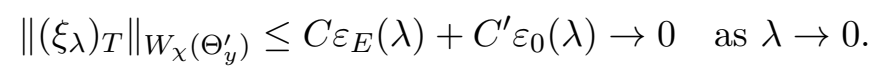

3.3.2. A useful normalizing function. The estimates for the longitudinal components hinge on the observation that, after certain normalization, $E_{w_{\chi}}$ behaves like the simple operator $d / d s$ over the longitudinal components.

Definition. Let $\ell(s)$ be the positive real function such that

$$
\begin{aligned}
\left\langle w_{\chi}^{\prime}, E_{w_{\chi}}\left(\ell \mathbf{e}_{w}\right)\right\rangle_{2, t} & =0 \quad \text { and } \ell\left(\gamma_{u_{0}}^{-1}(0)\right)=1, \text { where } \\
\mathbf{e}_{w}(s) & :=\left\|w_{\chi}^{\prime}\right\|_{2, t}^{-1}(s) w_{\chi}^{\prime}(s) .
\end{aligned}
$$


Lemma. The function $\ell$ is always positive, and there are positive constants $C_{1}, C_{2}$ independent of $\lambda$ such that

$$
0<C_{1} \leq \ell(s)\left\|w_{\chi}^{\prime}\right\|_{2, t}(s)^{-1} \leq C_{2} \quad \forall s \in\left[\gamma_{u_{0}}^{-1}(0), \gamma_{u_{k+1}}^{-1}(0)\right] .
$$

Furthermore, over $\left[\mathfrak{s}_{j-}, \mathfrak{s}_{j+}\right] \forall j$,

$$
0<C_{1} \lambda \leq \ell \leq C_{2} \lambda ; \quad\left|\ell^{\prime}\right| \leq C|\lambda|^{1 / 2}|\ell| .
$$

Proof. $\ell$ satisfies a first-order linear differential equation, so its existence and uniqueness is obvious. It also follows that $\ell$ has no zeros, because otherwise it would be identically zero. The condition that $\ell\left(\gamma_{u_{0}}^{-1}(0)\right)=1$ therefore implies that $\ell$ is always positive.

Equation (30) follows from the next claim by observing that $\ell\left\|w_{\chi}^{\prime}\right\|_{2, t}^{-1}\left(\gamma_{u_{0}}^{-1}(0)\right)$ is $\lambda$-independent.

Claim. Let $\mathfrak{s}_{0+}:=\gamma_{u_{0}}^{-1}(0) ; \mathfrak{s}_{k+2-}:=\gamma_{u_{k+1}}^{-1}(0)$. Then

$$
\left|\ln \left(\ell\left\|w_{\chi}^{\prime}\right\|_{2, t}\left(r_{1}\right)\right)-\ln \left(\ell\left\|w_{\chi}^{\prime}\right\|_{2, t}\left(r_{2}\right)\right)\right| \leq C
$$

for a constant $C$ independent of $r_{1}, r_{2}$, and $\lambda$ when $r_{1}, r_{2}$ are both in

(a) $\left[\mathfrak{s}_{i+}, \mathfrak{s}_{i+1}\right]$ for some $i \in\{0,1, \ldots, k+1\}$ or

(b) $\left[\mathfrak{s}_{j-}, \mathfrak{s}_{j+}\right]$ for some $j \in\{1,2, \ldots, k+1\}$.

Proof of the Claim. In case (a), set $u=u_{i}$ and drop the index $i$. In this case, $\left|\gamma_{u}\right| \leq C_{i} \lambda^{-1 / 2}, \gamma_{u}^{\prime}$ is close to 1 , and $\left\|w_{\chi}^{\prime}\right\|_{2, t}$ can be approximated by $\left\|u_{\gamma}\left(\gamma_{u}\right)\right\|_{2, t}$. We will therefore estimate $\ell\left\|u_{\gamma}\right\|_{2, t}^{-1}$ instead. In this region, rewrite (29) as

$$
\frac{d}{d s}\left(\ln \left(\ell\left\|u_{\gamma}\right\|_{2, t}^{-1}\right)\right)=\left(\gamma_{u}^{\prime}-1\right) \frac{d}{d \gamma}\left(\ln \left\|u_{\gamma}\right\|_{2, t}^{-1}\right)
$$

and integrate over $s$. Using the estimates in Section 2, it is easy to see that in this region, the $L^{\infty}$-norm of the right-hand side of the above equation can be bounded by $C \lambda|\gamma| \leq C^{\prime} \lambda^{1 / 2}$. On the other hand, the distance between $r_{1}$ and $r_{2}$ can be bounded by a multiple of $\lambda^{-1 / 2}$, so the claim is verified in this case.

In case (b), set $u=u_{j-1}$ or $u_{j}$ depending on whether $s$ is smaller or larger than $\mathfrak{l}_{j}$, and again drop the index $j-1$ or $j$. In this case, $\left|\gamma_{u}\right| \geq C_{i} \lambda^{-1 / 2}$, and $\lambda\left\|w_{\chi}^{\prime}\right\|_{2, t}^{-1}$ can be bounded above and below independently of $\lambda$ (cf. (27)) in this region, so it suffices to estimate the variation in $\ell$. We write (29) in the form:

$$
\frac{d}{d s}(\ln \ell)=-\left\|u_{\gamma}\right\|_{2, t}^{-1}\left(u_{\gamma \gamma}\right)_{L}
$$


in this case and again integrate over $s$. The claim then follows from the bound

$$
\left|\frac{d}{d s}(\ln \ell)\right| \leq C_{3}\left|\gamma_{u}\right|^{-1} \leq C_{3}^{\prime} \lambda^{1 / 2}
$$

and bound of the distance between $r_{1}$ and $r_{2}$ can be bounded by $C_{4} \lambda^{-1 / 2}$.

Continuing the proof of the lemma, the first inequality in (31) is the consequence of (30) and (27). The second inequality of (31) follows directly from (32) and the first inequality.

3.3.3. Estimating the longitudinal direction. It is convenient to introduce the following.

Definition. For $j=1, \ldots, k+1$, let the $\mathbb{R}$-valued function $f_{j}(s)$ be the unique solution of

$$
\begin{gathered}
E_{w_{\chi}}\left(f_{j} \ell \mathbf{e}_{w}\right)=f_{j}^{\prime} \ell \mathbf{e}_{w}=\mathfrak{f}_{j}, \\
\ell f_{j}\left(\mathfrak{s}_{j-}\right)=0 .
\end{gathered}
$$

Also, let $\phi_{\lambda}(s), \psi_{\lambda, i}(\gamma)$ be the $\mathbb{R}$-valued functions defined, respectively, by

$$
\begin{aligned}
\ell \phi_{\lambda}(s) & =\left\langle\xi_{\lambda}(s), \mathbf{e}_{w}(s)\right\rangle_{2, t}, \\
\psi_{\lambda, i}(\gamma) & =\left\langle\mathcal{T}_{w_{\chi}, \underline{w}_{\chi}}^{\gamma_{u_{i}}} \xi_{\lambda}(\gamma), \mathbf{e}_{u_{i}}(\gamma)\right\rangle_{2, t}, \quad \text { where } \mathbf{e}_{u_{i}}=\left\|u_{i}^{\prime}\right\|_{2, t}^{-1} u_{i}^{\prime} .
\end{aligned}
$$

The estimates for the longitudinal components will be based on the following elementary lemma.

Lemma. If $q \in L_{1}^{p}([0, l])$, then

(a) $\|q\|_{\infty} \leq C_{1} l^{1-1 / p}\left\|q^{\prime}\right\|_{p}+C_{2} l^{-1 / p}\|q\|_{p}$.

If furthermore $q(0)=0$, then in addition:

(b) $\|q\|_{\infty} \leq C l^{1-1 / p}\left\|q^{\prime}\right\|_{p}$;

(c) $\|q\|_{p} \leq C^{\prime} l\left\|q^{\prime}\right\|_{p}$.

The positive constants $C, C^{\prime}, C_{1}, C_{2}$ are independent of $q$ and $l$.

Let $\bar{\phi}_{\lambda, j}:=\phi_{\lambda}+\iota_{\lambda, j} f_{j}$. Then by (31), (27), (21), and part (c) of the above lemma,

$$
\begin{aligned}
& \left\|\left(\xi_{\lambda}\right)_{L}+\iota_{\lambda, j} \ell f_{j} \mathbf{e}_{w}\right\|_{W_{\chi}\left(\Theta_{y j}\right)} \\
\leq & C_{1}\left(\lambda^{1 / 2}\left\|\bar{\phi}_{\lambda, j}\right\|_{L^{p}\left(\Theta_{y j}\right)}+\left\|\bar{\phi}_{\lambda, j}^{\prime}\right\|_{L^{p}\left(\Theta_{y, j}\right)}\right) \\
\leq & C_{2}\left(\epsilon^{-1 / 2}\left\|\bar{\phi}_{\lambda, j}^{\prime}\right\|_{L^{p}\left(\Theta_{y, j}\right)}+\lambda^{-1 / 2-1 /(2 p)} \epsilon^{-1 /(2 p)}\left|\psi_{\lambda, j-1}\right|\left(\mathfrak{r}_{j-1}\right)\right) \\
\leq & C_{3}\left(\epsilon^{-1 / 2}\left\|\left(\tilde{E}_{\chi} \tilde{\xi}_{\lambda L}\right)_{L}\right\|_{L_{\chi}\left(\Theta_{y j}\right)}+\lambda^{-1 / 2-1 /(2 p)} \epsilon^{-1 /(2 p)}\left|\psi_{\lambda, j-1}\right|\left(\mathfrak{r}_{j-1}\right)\right) \\
\leq & C_{4}\left(\epsilon^{-1 / 2} \varepsilon_{E}+\epsilon^{-1 / 2-1 /(2 p)} \lambda^{1 / 2-1 /(2 p)} \varepsilon_{0}\right) \\
& \left.\quad+\lambda^{-1 / 2-1 /(2 p)} \epsilon^{-1 /(2 p)}\left|\psi_{\lambda, j-1}\right|\left(\mathfrak{r}_{j-1}\right)\right) .
\end{aligned}
$$


In the last line above, $\varepsilon_{0}$ comes from Lemma 3.3.1 and an estimate for $\left(E_{w_{\chi}} \xi_{\lambda T}\right)_{L}$ via a computation similar to I.(47). To estimate the last term above, note that from Lemma 3.2.1, we have

$$
\begin{aligned}
& \left\|E_{u_{i}}\left(\psi_{\lambda, i} \mathbf{e}_{u_{i}}\right)\right\|_{L_{u_{i}}\left(\left[0, \mathfrak{r}_{i}\right] \times S^{1}\right)} \\
& \quad \leq(1+2 \epsilon)\left\|\tilde{E}_{\chi}\left(\tilde{\xi}_{\lambda}\right)\right\|_{L_{\chi}}+(C \lambda+\epsilon)\left\|\xi_{\lambda L}\right\|_{W_{\chi}}+C^{\prime}\left\|\mathcal{T}_{w_{\chi}, \underline{w}_{\chi}}^{\gamma_{u_{i}}} \xi_{\lambda T}\right\|_{W_{u_{i}}\left(\left[0, \mathfrak{r}_{i}\right] \times S^{1}\right)} \\
& \quad \leq C \varepsilon_{u_{i}}^{\prime} \rightarrow 0 \quad \text { as } \lambda \rightarrow 0 .
\end{aligned}
$$

In the above, we used (26) to estimate $\left\|\mathcal{T}_{w_{\chi}, \underline{w}_{\chi}}^{\gamma_{u_{i}}} \xi_{T}\right\|_{W_{u_{i}}\left(\left[0, \mathfrak{r}_{i}\right] \times S^{1}\right)}$. On the other hand,

$$
\left\|E_{u_{i}}\left(\psi_{\lambda, i} \mathbf{e}_{u_{i}}\right)\right\|_{L_{u_{i}}\left(\left[0, \mathfrak{r}_{i}\right] \times S^{1}\right)} \geq C \|\left(\sigma_{u_{i}}\left(\psi_{\lambda, i}\right)_{\gamma} \|_{L^{p}\left(\left[0, \mathfrak{r}_{i}\right]\right)} .\right.
$$

Using Lemma 3.3.3 (b) and the fact that $\psi_{\lambda, i}(0)=0$ (because $\xi_{\lambda} \in W_{\chi}^{\prime}$ ), the previous two inequalities imply:

$$
\begin{aligned}
\left|\psi_{\lambda, i}\left(\mathfrak{r}_{i}\right)\right| & \leq C \lambda \mathfrak{r}_{i}^{1-1 / p} \varepsilon_{u_{i}}^{\prime} \\
& \leq C^{\prime} \lambda^{1 / 2+1 /(2 p)} \epsilon^{1 / 2-1 /(2 p)} \varepsilon_{u_{i}}^{\prime} .
\end{aligned}
$$

Combining this with (34), we have

$$
\begin{aligned}
& \left\|\left(\xi_{\lambda}\right)_{L}+\iota_{\lambda, j} \ell f_{j} \mathbf{e}_{w}\right\|_{W_{\chi}\left(\Theta_{y j}\right)} \\
& \quad \leq C_{5}\left(\epsilon^{-1 / 2} \varepsilon_{E}+\epsilon^{-1 / 2-1 /(2 p)} \lambda^{1 / 2-1 /(2 p)} \varepsilon_{0}+\epsilon^{1 / 2-1 / p} \varepsilon_{u_{j-1}}^{\prime}\right) .
\end{aligned}
$$

3.4. Estimating $\iota_{j}$ and $f_{j}$. This subsection fills in the last ingredients for the proof of Proposition 3.1.3: estimates for $\iota_{j}$ and $f_{j}$ (Lemmas 3.4.1 and 3.4.2 respectively). Combining these estimates with the estimates obtained in previous subsections, we finish the proof of Proposition 3.1.3 in Section 3.4.3.

3.4.1. Lemma. Let $\lambda, \epsilon$ be small positive numbers as before. Then

$$
\left|\iota_{\lambda, j}\right| \leq \varepsilon_{\iota, j}(\lambda, \epsilon)
$$

where $\varepsilon_{\iota, j}>0$ can be made arbitrarily small as $\lambda \rightarrow 0$ by choosing $\epsilon$ appropriately.

Proof. This lemma follows from a lower bound on $\ell f_{j}\left(\mathfrak{s}_{j+}\right)$, and a upper bound on $\iota_{\lambda, j} \ell f_{j}\left(\mathfrak{s}_{j+}\right)$, given, respectively, in (37), (38) below.

Note from the defining equation for $f_{j}$ and (31) that $\left|f_{j}^{\prime}\right| \geq C \lambda^{1 /(2 p)}$ for some $\lambda$-independent constant $C$. Therefore by (21), (31), and the initial condition of $f_{j}(33)$,

$$
\begin{aligned}
\ell f_{j}\left(\mathfrak{s}_{j+}\right) & =\ell\left(\mathfrak{s}_{j+}\right)\left(f_{j}\left(\mathfrak{s}_{j+}\right)-f_{j}\left(\mathfrak{s}_{j-}\right)\right) \\
& \geq C_{1} \lambda^{1+1 /(2 p)}\left(\mathfrak{s}_{j+}-\mathfrak{s}_{j-}\right) \\
& \geq C_{j} \epsilon^{-1 / 2} \lambda^{1 / 2+1 /(2 p)} .
\end{aligned}
$$


A similar calculation establishes an analogous upper bound, and we have

$$
C_{j}^{\prime} \epsilon^{-1 / 2} \lambda^{1 / 2+1 /(2 p)} \geq \ell f_{j}\left(\mathfrak{s}_{j+}\right) \geq C_{j} \epsilon^{-1 / 2} \lambda^{1 / 2+1 /(2 p)} .
$$

On the other hand,

$$
\left|\iota_{\lambda, j}\right| \ell f_{j}\left(\mathfrak{s}_{j+}\right) \leq\left|\psi_{\lambda, j-1}\left(\mathfrak{r}_{j-1}\right)\right|+\left|-\psi_{\lambda, j}\left(\mathfrak{r}_{j}\right)\right|+\left|\ell \bar{\phi}_{\lambda}\left(\mathfrak{s}_{j+}\right)-\ell \bar{\phi}_{\lambda}\left(\mathfrak{s}_{j-}\right)\right| .
$$

The first two terms on the RHS are already estimated in (35); the third term can be bounded by

$$
\left|\ell\left(\mathfrak{s}_{j+}\right)\left(\bar{\phi}_{\lambda}\left(\mathfrak{s}_{j+}\right)-\bar{\phi}_{\lambda}\left(\mathfrak{s}_{j-}\right)\right)\right|+\left|\left(\ell\left(\mathfrak{s}_{j+}\right)-\ell\left(\mathfrak{s}_{j-}\right)\right) \bar{\phi}_{\lambda}\left(\mathfrak{s}_{j-}\right)\right|,
$$

in which the first term may be bounded via (31), Lemma 3.3.3 (b) by

$$
\begin{aligned}
& C \lambda(\epsilon \lambda)^{-1 / 2+1 /(2 p)}\left\|\bar{\phi}_{\lambda}^{\prime}\right\|_{L^{p}\left(\left[\mathfrak{s}_{j-}, \mathfrak{s}_{j+}\right]\right)} \\
& \quad \leq C^{\prime} \lambda^{1 / 2+1 /(2 p)}\left(\epsilon^{-1 / 2+1 /(2 p)} \varepsilon_{E}+\epsilon^{-1 / 2} \lambda^{1 / 2-1 /(2 p)} \varepsilon_{0}\right),
\end{aligned}
$$

according to the computation in (34), lines 3-5.

The second term, via (35), the initial condition (33), and (31), may be bounded by

$$
C^{\prime \prime} \lambda^{1 / 2+1 /(2 p)} \epsilon^{1 / 2-1 /(2 p)} \varepsilon_{u_{j-1}}^{\prime} .
$$

Summing up, we have

$$
\begin{aligned}
& \left|\iota_{\lambda, j}\right| \ell f_{j}\left(\mathfrak{s}_{j+}\right) \leq C_{0} \epsilon^{-1 / 2} \lambda^{1 / 2+1 /(2 p)} . \\
& \quad\left(\epsilon^{1 /(2 p)} \varepsilon_{E}+(\lambda+\epsilon) \epsilon^{1-1 /(2 p)}+\varepsilon_{0}\left(\lambda^{1 / 2-1 /(2 p)}+\epsilon^{1-1 /(2 p)}\right)\right) .
\end{aligned}
$$

Comparing with (37), we have an estimate for $\iota_{\lambda, j}$ as asserted in the lemma, with

$$
\varepsilon(\epsilon, \lambda)=C_{1}\left(\epsilon^{1 /(2 p)} \varepsilon_{E}+(\lambda+\epsilon) \epsilon^{1-1 /(2 p)}+\varepsilon_{0}\left(\lambda^{1 / 2-1 /(2 p)}+\epsilon^{1-1 /(2 p)}\right)\right),
$$

which can be made arbitrarily small by choosing $\lambda, \epsilon$ appropriately.

3.4.2. Lemma. For $j=1, \ldots, k+1$,

$$
\lambda^{-1 / 2}\left\|\ell f_{j} \mathbf{e}_{w}\right\|_{L^{p}\left(\Theta_{y j}\right)}+\lambda^{-1}\left\|\left(\ell f_{j}\right)^{\prime} \mathbf{e}_{w}\right\|_{L^{p}\left(\Theta_{y j}\right)} \leq C_{j}^{\prime \prime} \epsilon^{-1 / 2-1 /(2 p)} .
$$

Proof. Note that since $f_{j}^{\prime}=\ell^{-1}\left\langle\mathbf{e}_{w}, \mathfrak{f}_{j}\right\rangle_{2, t}>0, f_{j}$ is increasing, and thus the estimates leading to (37) imply that

$$
\left\|\ell f_{j}\right\|_{L^{\infty}\left(\Theta_{y j}\right)} \leq C_{1} \epsilon^{-1 / 2} \lambda^{1 / 2+1 /(2 p)} .
$$

On the other hand, this and (31) yield

$$
\begin{aligned}
\left\|\left(\ell f_{j}\right)^{\prime}\right\|_{L^{\infty}\left(\Theta_{y j}\right)} & \leq\left\|\ell f_{j}^{\prime}\right\|_{L^{\infty}\left(\Theta_{y j}\right)}+\left\|\ell^{\prime} f_{y j}\right\|_{L^{\infty}\left(\Theta_{y j}\right)} \\
& \leq C_{2}\left(\lambda^{1+1 /(2 p)}+\lambda^{1 / 2}\left\|\ell f_{j}\right\|_{L^{\infty}\left(\Theta_{y j}\right)}\right) \\
& \leq C_{3} \epsilon^{-1 / 2} \lambda^{1+1 /(2 p)} .
\end{aligned}
$$

These two $L^{\infty}$-bounds together with the length estimate for $\Theta_{y j}$ imply the lemma. 
3.4.3. Concluding the proof of Proposition 3.1.3. Now we have all the ingredients to finish the proof of the proposition.

By Lemmas 3.4.1 and 3.4.2 the $\iota$-terms in (36) are ignorable as $\lambda \rightarrow 0$. They are bounded by expressions of the form

$$
C_{1}\left(\varepsilon_{E} \epsilon^{-1 / 2}+(\lambda+\epsilon) \epsilon^{1 / 2-1 / p}+\varepsilon_{0}\left(\lambda^{1 / 2-1 /(2 p)} \epsilon^{-1 / 2-1 /(2 p)}+\epsilon^{1 / 2-1 / p}\right)\right),
$$

which can be made arbitrarily small by requiring, e.g.,

$$
\begin{aligned}
& \lambda=\lambda(\epsilon) \text { is small enough such that } \\
& \lambda<\epsilon^{5}, \varepsilon_{E}(\lambda)+\varepsilon_{0}(\lambda)<\epsilon^{3}, \text { and } \\
& \epsilon \rightarrow 0 .
\end{aligned}
$$

Applying the comparison Lemma 3.2.1 to (26) for all $i$, and adding them to $(28)$ and (36) for all $j$, we see that

$$
\left\|\xi_{\lambda}\right\|_{W_{\chi}} \leq C_{4}\left(\epsilon^{-1 / 2} \varepsilon_{E}+\lambda+\epsilon+\varepsilon_{0}\left(1+\epsilon^{-1 / 2-1 /(2 p)} \lambda^{1 / 2-1 /(2 p)}\right)\right) \ll 1
$$

by the same choice (39). Combining this with Lemma 3.4.1, we arrive at a contradiction to $(22)$.

\section{Gluing at Deaths III: the gluing map}

Sections 4.1 and 4.2 finish the proof of Proposition 2.1 (a): by further analyzing the Kuranishi map associated to the K-model of last section, we obtain a smooth gluing map, which is a local diffeomorphism. We show that this gluing map surjects to a neighborhood of the stratum $\mathbb{S}$ in Section 4.2.

In Section 4.3, we discuss the minor modification needed to obtain part (b) of Proposition 2.1, which glues broken orbits at $\lambda=0$ to create new closed orbits for small $\lambda>0$.

4.1. Understanding the Kuranishi map. In the previous section, we constructed the K-model for the family of deformation operators, $\left[K^{\Xi} \rightarrow\right.$ $\left.C^{\Xi}\right]$. According to the discussion in Section 1.2.6, this yields a local description of the moduli space as the zero locus of an analytic map. In this subsection, we analyze this analytic map in more detail; this analysis enables us to show that the moduli space is in fact (Zariski) smooth.

Recall that Proposition 3.1.3 shows that we have decompositions:

$$
W_{\chi}=\bigoplus_{i} \mathbb{R e}_{u_{i}} \oplus W_{\chi}^{\prime}, \quad L_{\chi}=\bigoplus_{j} \mathbb{R f}_{j} \oplus E_{w_{\chi}}\left(W_{\chi}^{\prime}\right),
$$

and the (nonorthogonal) projection of $L_{\chi}$ to the $\mathfrak{f}_{j}$ direction is given by

$$
P_{\mathfrak{f}_{j}}=\Pi_{j} \tilde{G}_{\chi},
$$

where $\Pi_{j}$ is the projection to the $j$ th $\mathbb{R}$-component of $\tilde{W}_{\chi}$. By Proposition 3.1.3, $P_{f_{j}}$ has uniformly bounded operator norm. However, we need a finer 
estimate for $P_{f_{j}}$ to understand the Kuranishi map. The next lemma is a useful tool for this purpose.

4.1.1. Lemma (Projection via integration). Let $\eta \in L_{\chi}$, and as usual denotes

$$
\underline{\eta}_{L}(s):=\left\|w_{\chi}^{\prime}(s)\right\|_{2, t}^{-1}\left\langle w_{\chi}^{\prime}(s), \eta(s)\right\rangle_{2, t} \quad \text { for } s \in\left[\gamma_{u_{0}}^{-1}(0), \gamma_{u_{k+1}}^{-1}(0)\right] .
$$

Then the projection $P_{f_{j}} \eta$ is bounded above and below by expressions of the form

$$
C_{1 \pm} \lambda^{1 / 2-1 /(2 p)} \int_{\gamma_{u_{j-1}}^{-1}(0)}^{\gamma_{u_{j}}^{-1}(0)} \ell^{-1} \underline{\eta}_{L} d s-C_{2 \pm} \lambda^{1 / 2-1 /(2 p)}\|\eta\|_{L_{\chi}}
$$

for $\lambda$-independent constants $C_{1 \pm}, C_{2 \pm}$.

In our later applications of this lemma, the second term in the above expression is typically dominated by the first term, and hence ignorable.

Proof. In accordance with decomposition (40), write

$$
\eta=E_{w_{\chi}} \xi+\sum_{j=1}^{k+1} \iota_{j} \mathfrak{f}_{j}
$$

for $\left(\iota_{1}, \ldots, \iota_{k+1}, \xi\right) \in \tilde{W}_{\chi}$. Thus

$$
\begin{aligned}
& \int_{\mathrm{S}_{j-}}^{\mathrm{S}_{j+}} \ell^{-1} d s \iota_{j} \\
= & C_{j}^{-1} \lambda^{-1-1 /(2 p)}\left(\int_{\gamma_{u_{j-1}}^{-1}(0)}^{\gamma_{u_{j}}^{-1}(0)} \ell^{-1} \underline{\eta} \underline{ }_{L} d s-\int_{\gamma_{u_{j-1}}^{-1}(0)}^{\gamma_{u_{j}}^{-1}(0)} \ell^{-1} \underline{\left(E_{w_{\chi}} \xi\right)} L d s\right) \\
= & C_{j}^{-1} \lambda^{-1-1 /(2 p)}\left(\int_{\gamma_{u_{j-1}}^{-1}(0)}^{\gamma_{u_{j}}^{-1}(0)} \ell^{-1} \underline{\eta}_{L} d s-\int_{\gamma_{u_{j-1}}^{-1}(0)}^{\gamma_{u_{j}}^{-1}(0)} \ell^{-1} \frac{\left(E_{w_{\chi}} \xi_{T}\right)}{L} d s\right) .
\end{aligned}
$$

The second identity above is due to the fact that $\xi_{L}\left(\gamma_{u_{j-1}}^{-1}(0)\right)=0=$ $\xi_{L}\left(\gamma_{u_{j}}^{-1}(0)\right)$ : writing $\xi_{L}(s)=\ell \phi w_{\chi}^{\prime}\left\|w_{\chi}^{\prime}\right\|_{2, t}^{-1}(s)$, we see from the definition of $\ell$ that $\ell^{-1}\left(E_{w_{\chi}} \xi_{L}\right)_{L}=\phi^{\prime}$. Now integrate by parts, using the fact that since $\ell(s) \neq 0 \forall s, \phi\left(\gamma_{u_{j-1}}^{-1}(0)\right)=0=\phi\left(\gamma_{u_{j}}^{-1}(0)\right)$.

By (31) and (21), we have

$$
C_{h} \lambda^{-3 / 2} \leq \int_{\mathrm{S}_{j-}}^{\mathrm{s}_{j+}} \ell^{-1} d s \leq C_{h}^{\prime} \lambda^{-3 / 2}
$$

on the other hand, a computation similar to that leading to I.(47) yields

$$
\begin{aligned}
& \left|\ell^{-1} \underline{\left(E_{w_{\chi}} \xi_{T}\right)}\right|(s) \leq C_{1}\left(\sigma_{\chi}\left\|u_{\gamma}\right\|_{2, t}^{-1}\left(\gamma_{u}^{\prime}+1\right)\left\|\left(u_{\gamma \gamma}\right)_{T}\right\|_{2, t}+\lambda\right)\left\|\xi_{T}\right\|_{2, t}(s) \\
& \leq C_{2}\left\|\xi_{T}\right\|_{2, t}(s) \text {, }
\end{aligned}
$$


where $u=u_{j-1}$ or $u_{j}$ depending on whether $s<\mathfrak{l}_{j}$ or $>\mathfrak{l}_{j}$. So by the estimates for $\left\|w_{\chi}^{\prime}\right\|_{2, t}$ and $\gamma_{u_{i}}^{-1}(0)-\gamma_{u_{i-1}}^{-1}(0)$, and the uniform boundedness of $\tilde{G}_{\chi}$,

$$
\begin{aligned}
& \left|\int_{\gamma_{u_{j-1}}^{-1}(0)}^{\gamma_{u_{j}}^{-1}(0)} \ell^{-1} \frac{\left(E_{w_{\chi}} \xi_{T}\right)}{} L d s\right| \\
& \quad \leq C_{3}\left\|\sigma_{\chi}^{-1 / 2}\right\|_{L^{q}\left(\left(\gamma_{u_{j-1}}^{-1}(0), \gamma_{u_{j}}^{-1}(0)\right) \times S^{1}\right)}\left\|\xi_{T}\right\|_{W_{\chi}} \leq C_{4}\|\eta\|_{L_{\chi}},
\end{aligned}
$$

where $q^{-1}:=1-p^{-1}$. Putting (43), (44), (45) together, we arrive at (41).

Next, applying the recipe of Section 1.2.5 to the K-models given by Proposition 3.1.3, we look for solutions $\left(\alpha, \varphi_{0}, \ldots, \varphi_{k+1}, \xi\right) \in W_{\chi}^{\prime} \oplus \mathbb{R} \oplus \mathbb{R}^{k+2}$ of

$$
\begin{aligned}
& P^{c}\left(\bar{\partial}_{J X_{\lambda}} w_{\chi}+\hat{E}_{\left(\lambda, w_{\chi}\right)}(\alpha, \xi)\right. \\
& \left.\quad+\sum_{i=0}^{k+1} \varphi_{i} E_{w_{\chi}} \mathfrak{e}_{u_{i}}+\hat{n}_{\left(\lambda, w_{\chi}\right)}\left(\alpha, \xi+\sum_{i=0}^{k+1} \varphi_{i} \mathfrak{e}_{u_{i}}\right)\right)=0 ; \\
& P_{\mathrm{f}_{j}}\left(\bar{\partial}_{J X_{\lambda}} w_{\chi}+\hat{E}_{\left(\lambda, w_{\chi}\right)}\left(\alpha, \sum_{i=0}^{k+1} \varphi_{i} \mathfrak{e}_{u_{i}}\right)\right. \\
& \left.\quad+\hat{n}_{\left(\lambda, w_{\chi}\right)}\left(\alpha, \xi+\sum_{i=0}^{k+1} \varphi_{i} \mathfrak{e}_{u_{i}}\right)\right)=0,
\end{aligned}
$$

where $P^{c}:=1-\sum_{j} P_{\mathrm{f}_{j}}$.

\subsubsection{Lemma (Solving the infinite-dimensional equation). Given}

$\hat{\varphi}:=\left(\alpha, \varphi_{0}, \ldots, \varphi_{k+1}\right) \in \mathbb{R} \oplus \mathbb{R}^{k+2}, \quad$ with $|\hat{\varphi}|^{2}:=\left|\lambda^{-3 / 2} \alpha\right|^{2}+\sum_{i}\left|\varphi_{i}\right|^{2} \ll 1$,

(46) has a unique solution $\xi(\hat{\varphi})$, with

$$
\|\xi(\hat{\varphi})\|_{W_{\chi}} \leq C_{1} \lambda^{1 / 2-1 /(2 p)}+C_{2} \lambda^{1 / 2-1 /(2 p)}|\hat{\varphi}|+C_{3} \lambda^{1-1 /(2 p)}|\hat{\varphi}|^{2} .
$$

Furthermore, the solution $\xi\left(\hat{\varphi}^{\prime}\right)$ corresponding to another $\hat{\varphi}^{\prime}=\left(\alpha^{\prime}, \varphi_{0}^{\prime}, \ldots\right.$, $\left.\varphi_{k+1}^{\prime}\right)$ satisfies

$$
\left\|\xi(\hat{\varphi})-\xi\left(\hat{\varphi}^{\prime}\right)\right\|_{W_{\chi}} \leq C_{4} \lambda^{1 / 2-1 /(2 p)}\left|\hat{\varphi}-\hat{\varphi}^{\prime}\right| .
$$

The significance of the factor $|\lambda|^{2 / 3}$ associated to $\alpha$ in the definition of $|\hat{\varphi}|$ will become clear in (70).

Proof. To apply the usual contraction mapping argument (Lemma 1.2.1) to (46), we need to estimate the "error" $\mathcal{F}$ and the nonlinear term $N$, and to show that the linearization has a uniformly bounded right inverse. 
In this context, the error $\mathcal{F}$ consists of

$$
P^{c} \bar{\partial}_{J X_{\lambda}} w_{\chi}+P^{c} \sum_{i=0}^{k+1} \varphi_{i} E_{w_{\chi}} \mathfrak{e}_{u_{i}}+\alpha P^{c} Y_{\left(\lambda, w_{\chi}\right)}+P^{c} \hat{n}_{\left(\lambda, w_{\chi}\right)}\left(\alpha, \sum_{i=0}^{k+1} \varphi_{i} \mathfrak{e}_{u_{i}}\right),
$$

which we estimate term by term below. We shall drop all $P^{c}$ from the terms, since by Proposition 3.1.3, it has uniformly bounded operator norm, and thus only affect the estimate by a $\lambda$-independent factor.

For the first term, note that $\left\|\bar{\partial}_{J X_{\lambda}} w_{\chi}\right\|_{L_{\chi}}$ is readily estimated by Proposition 2.4.1.

For the second term, we claim:

$$
\left\|E_{w_{\chi}} \gamma_{u_{i}}^{*}\left(u_{i}\right)_{\gamma}\right\|_{L_{\chi}} \leq C \lambda^{1 / 2-1 /(2 p)} .
$$

We shall again suppress the subscript $i$ below. Note that

$$
E_{w_{\chi}} \gamma_{u}^{*} u_{\gamma}=\left(\gamma_{u}^{\prime}-1\right) u_{\gamma \gamma}\left(\gamma_{u}\right)+Z(u) u_{\gamma}\left(\gamma_{u}\right)
$$

where $Z$ arises from the difference between $X_{\lambda}$ and $X_{0}$, and hence we have $\|Z(u)\|_{\infty} \leq C \lambda$. Thus, by I.5.3.1 (2c) and routine estimates, the $L_{\chi}$-norm of the second term above is also bounded by $C^{\prime} \lambda$.

For the first term, note that the length of $\left[\gamma_{u}^{-1}\left(-\gamma_{0}\right), \gamma_{u}^{-1}\left(\gamma_{0}\right)\right]$ is bounded independently of $\lambda$; therefore, the $L_{\chi}$-norm of it in this region is bounded by $C \lambda$. On the other hand, the length of the intervals where $\left|\gamma_{u}\right| \geq \gamma_{0}$ is bounded by $C^{\prime} \lambda^{-1 / 2}$. When $s$ is in these intervals, by the computations in case (2) in proof of Lemma 2.4.2, $\left\|\sigma_{\chi}\left(\gamma_{u}^{\prime}-1\right) u_{\gamma \gamma}\left(\gamma_{u}\right)\right\|_{\infty} \leq C_{3} \lambda^{1 / 2}$. Together with the length estimate above, we see that the $L_{\chi}$-norm in this region is bounded by $C^{\prime \prime} \lambda^{1 / 2-1 /(2 p)}$. Equation (50) is verified.

For the third term, recall the following estimate obtained in the proof of Lemma 2.5.1:

$$
\left\|\alpha Y_{\left(\lambda, w_{\chi}\right)}\right\|_{L_{\chi}} \leq C\|(\alpha, 0)\|_{\hat{W}_{\chi}} \leq C \lambda^{1 / 2-1 /(2 p)}|\hat{\varphi}| .
$$

For the last term in the error, we have

$$
\begin{aligned}
& \quad\left\|\hat{n}_{\left(\lambda, w_{\chi}\right)}\left(\alpha, \sum_{i=0}^{k+1} \varphi_{i} \mathfrak{e}_{u_{i}}\right)\right\|_{L_{\chi}} ; \\
& \leq C_{2} \sum_{i=0}^{k+1}\left(\left\|n_{w_{\chi}}\left(\varphi_{i} \mathfrak{e}_{u_{i}}\right)\right\|_{L_{\chi}}+\left\|\alpha \varphi_{i} \nabla_{\mathrm{e}_{u_{i}}} Y_{\left(\lambda, w_{\chi}\right)}\right\|_{L_{\chi}}\right) \\
& \quad+C_{2}^{\prime} \alpha^{2}\left\|\partial_{\lambda} Y_{\left(\lambda, w_{\chi}\right)}\right\|_{L_{\chi}}+\text { higher order terms } \\
& \leq C_{3} \sum_{i=0}^{k+1}\left(\lambda\left|\varphi_{i}\right|^{2}+|\alpha| \lambda^{-1 /(2 p)}\left|\varphi_{i}\right|\right)+C_{3}^{\prime}|\alpha|^{2} \lambda^{-1-1 /(2 p)} \\
& \leq C_{4} \lambda|\hat{\varphi}|^{2} .
\end{aligned}
$$


For the first inequality above, we used the fact that for different $i, j, \mathfrak{e}_{u_{i}}, \mathfrak{e}_{u_{j}}$ have disjoint supports. For the second inequality, we used the invariance of the flow equation under translation, which implies

$$
n_{u_{i}}^{J X_{0}}\left(\varphi_{i}\left(u_{i}\right)_{\gamma}\right)=0 \quad \forall \varphi_{i} \in \mathbb{R}
$$

Next, the linear term in (46) is of the form $E_{\chi}^{\prime} \xi$, where $E_{\chi}^{\prime}$ is $E_{w_{\chi}}$ perturbed by a term coming from $\hat{n}_{\left(\lambda, w_{\chi}\right)}\left(\alpha, \sum_{i=0}^{k+1} \varphi_{i} \mathfrak{e}_{u_{i}}+\xi\right)$, which has operator norm bounded by

$$
C\left(\sum_{i=0}^{k+1}\left|\varphi_{i}\right|+\lambda^{-1 / 2}|\alpha|\right) \leq C|\hat{\varphi}| .
$$

Thus, with the assumption that $|\hat{\varphi}| \ll 1, E_{\chi}^{\prime}$ is uniformly right-invertible as $E_{w_{\chi}}$ is. By contraction mapping theorem and the error estimates above, we have an $\xi(\hat{\varphi})$ satisfying (48).

The estimate for the nonlinear term is not very different from that in Lemma 2.5.2, which we shall omit.

Finally, to estimate $\xi-\xi^{\prime}$, where $\xi:=\xi(\hat{\varphi}) ; \xi^{\prime}:=\xi\left(\hat{\varphi}^{\prime}\right)$, notice that it satisfies

$$
\begin{aligned}
E_{w_{\chi}}\left(\xi-\xi^{\prime}\right)= & -P^{c}\left(\sum_{i=0}^{k+1}\left(\varphi_{i}-\varphi_{i}^{\prime}\right) E_{w_{\chi}} \mathfrak{e}_{u_{i}}\right)+\left(\alpha-\alpha^{\prime}\right) Y_{\left(\lambda, w_{\chi}\right)} \\
& \left.+\hat{n}_{\left(\lambda, w_{\chi}\right)}\left(\alpha, \xi+\sum_{i=0}^{k+1} \varphi_{i} \mathfrak{e}_{u_{i}}\right)-\hat{n}_{\left(\lambda, w_{\chi}\right)}\left(\alpha^{\prime}, \xi^{\prime}+\sum_{i=0}^{k+1} \varphi_{i}^{\prime} \mathfrak{e}_{u_{i}}\right)\right) .
\end{aligned}
$$

Thus, by Proposition 3.1.3,

$$
\begin{aligned}
\left\|\xi-\xi^{\prime}\right\|_{W_{\chi}} \leq & C^{\prime}\left(\sum_{i=0}^{k+1}\left|\varphi_{i}-\varphi_{i}^{\prime}\right|\left\|E_{w_{\chi}} \mathfrak{e}_{u_{i}}\right\|_{L_{\chi}}+\left|\alpha-\alpha^{\prime}\right|\left\|Y_{\left(\lambda, w_{\chi}\right)}\right\|_{L_{\chi}}\right. \\
& \left.+\left\|\hat{n}_{\left(\lambda, w_{\chi}\right)}\left(\alpha, \xi+\sum_{i=0}^{k+1} \varphi_{i} \mathfrak{e}_{u_{i}}\right)-\hat{n}_{\left(\lambda, w_{\chi}\right)}\left(\alpha, \xi^{\prime}+\sum_{i=0}^{k+1} \varphi_{i}^{\prime} \mathfrak{e}_{u_{i}}\right)\right\|_{L_{\chi}}\right)
\end{aligned}
$$

for a $\lambda$-independent constant $C^{\prime}$. The first two terms inside the parenthesis may be bounded by $C_{1} \lambda^{1 / 2-1 /(2 p)}\left|\hat{\varphi}-\hat{\varphi}^{\prime}\right|$ according to (50) and (52). 
The third term, by direct computation and (53) again, may be bounded by

$$
\begin{aligned}
& \left(\begin{array}{c}
\|\xi\|_{W_{\chi}}+\left\|\xi^{\prime}\right\|_{W_{\chi}}+\left(|\alpha|+\left|\alpha^{\prime}\right|\right) \lambda^{-1 / 2} \\
+\sum_{i=0}^{k+1}\left(\left|\varphi_{i}\right|+\left|\varphi_{i}^{\prime}\right|\right)\left\|\mathfrak{e}_{u_{i}}\right\|_{W_{\chi}}
\end{array}\right) \cdot C_{2}\left\|\xi-\xi^{\prime}\right\|_{W_{\chi}} \\
& +\sum_{i=0}^{k+1}\left(\begin{array}{c}
\|\xi\|_{W_{\chi}}+\left\|\xi^{\prime}\right\|_{W_{\chi}}+\left(|\alpha|+\left|\alpha^{\prime}\right|\right) \lambda^{-1 / 2} \\
+\lambda\left(\left|\varphi_{i}\right|+\left|\varphi_{i}^{\prime}\right|\right)\left\|\mathfrak{e}_{u_{i}}\right\|_{W_{\chi}}
\end{array}\right) \cdot C_{3}\left\|\mathfrak{e}_{u_{i}}\right\|_{W_{\chi}}\left|\varphi_{i}-\varphi_{i}^{\prime}\right| \\
& +\left(\begin{array}{c}
\lambda^{-1 / 2}\left(\|\xi\|_{W_{\chi}}+\left\|\xi^{\prime}\right\|_{W_{\chi}}\right)+\lambda^{-1-1 /(2 p)}\left(|\alpha|+\left|\alpha^{\prime}\right|\right) \\
+\sum_{i=0}^{k+1}\left(\left|\varphi_{i}\right|+\left|\varphi_{i}^{\prime}\right|\right) \lambda^{-1 / 2}\left\|\mathfrak{e}_{u_{i}}\right\|_{W_{\chi}}
\end{array}\right) \cdot C_{4}\left|\alpha-\alpha^{\prime}\right| \\
& \leq C_{2}^{\prime} \varepsilon\left\|\xi-\xi^{\prime}\right\|_{W_{\chi}}+C_{3}^{\prime} \lambda^{1 / 2-1 /(2 p)}\left|\hat{\varphi}-\hat{\varphi}^{\prime}\right|,
\end{aligned}
$$

where $0<\varepsilon \ll 1$, and we have used (48) and the fact that $|\hat{\varphi}| \ll 1$ above. Now, the first term in the last expression above can be got rid of by a rearrangement argument, and we arrive at (49).

Next, substitute $\xi(\hat{\varphi})$ back in (47) to solve for $\hat{\varphi}$. To understand the behavior of the solutions, we estimate each term in the Kuranishi map in turn.

4.1.3. Lemma (Terms in the Kuranishi map). Let $q^{-1}:=1-p^{-1}$. Then:

(a) $\left|P_{f_{j}} \bar{\partial}_{J X_{\lambda}} w_{\chi}\right| \leq C^{\prime} \lambda^{1 / q}$ for any $j \in\{1, \ldots, k+1\}$;

(b) For any $i \in\{0, \ldots, k+1\}, j \in\{1, \ldots, k+1\}$,

$$
\begin{gathered}
\left|P_{f_{j}} E_{w_{\chi}} \mathfrak{e}_{u_{i}}\right| \leq C \lambda^{1 / q} \quad \text { if } j \neq i \text { or } i+1 ; \\
-C_{-}^{\prime} \lambda^{1 /(2 q)} \geq P_{f_{i}}\left(E_{w_{\chi}} \mathfrak{e}_{u_{i}}\right) \geq-C_{-} \lambda^{1 /(2 q)} ; \\
C_{+}^{\prime} \lambda^{1 /(2 q)} \geq P_{f_{i+1}}\left(E_{w_{\chi}} \mathfrak{e}_{u_{i}}\right) \geq C_{+} \lambda^{1 /(2 q)} .
\end{gathered}
$$

(c) Let $\hat{\varphi}, \hat{\varphi}^{\prime}, \xi:=\xi(\hat{\varphi}), \xi^{\prime}:=\xi\left(\hat{\varphi}^{\prime}\right)$ be as in the previous lemma. Then $\forall j$,

$$
\begin{aligned}
& \mid P_{f_{j}}\left(\hat{n}_{\left(\lambda, w_{\chi}\right)}\left(\alpha, \xi+\sum_{i=0}^{k+1} \varphi_{i} \mathfrak{e}_{u_{i}}\right)\right. \\
& \left.\quad-\hat{n}_{\left(\lambda, w_{\chi}\right)}\left(\alpha^{\prime}, \xi^{\prime}+\sum_{i=0}^{k+1} \varphi_{i}^{\prime} \mathfrak{e}_{u_{i}}\right)\right)\left|\leq C_{n} \lambda^{1 / q}\right| \hat{\varphi}-\hat{\varphi}^{\prime} \mid .
\end{aligned}
$$

Proof. (a) Apply (41) with $\eta=\bar{\partial}_{J X_{\lambda}} w_{\chi}$. The integrals in the first terms vanish because by our definition of pregluing, $\bar{\partial}_{J X_{\lambda}} w_{\chi}$ has no longitudinal 
component in this region. On the other hand, $\|\eta\|_{L_{\chi}} \leq C \lambda^{1 /(2 q)}$ by Proposition 2.4.1.

(b) Let $\eta=E_{w_{\chi}} \mathfrak{e}_{u_{i}}$ in (41). By (50), the second term of (41) (multiple of $\left.\lambda^{1 /(2 q)}\|\eta\|_{L_{\chi}}\right)$ contributes a multiple of $\lambda^{1 / q}$. If $j \neq i, i+1$, the first term of (41) (multiples of integrals) vanishes, because $\eta$ is supported away from the interval of integration. These together imply the first line of (b).

For the other cases $\left(u=u_{j}\right.$ or $\left.u_{j-1}\right)$, we shall show that

$$
\pm C^{\prime} \leq \int_{\gamma_{u_{j-1}}^{-1}(0)}^{\gamma_{u_{j}}^{-1}(0)} \ell^{-1} \frac{E_{w_{\chi}} \mathfrak{e}_{u}}{} \leq \pm C \quad\left(- \text { when } u=u_{j} \text {, + when } u=u_{j-1}\right) .
$$

This would imply that the first term of (41) is bounded below and above by positive multiples of $\pm \lambda^{1 /(2 q)}$, dominating the second term. The other two cases of (b) would then follow.

To see this, recall the computation of $E_{w_{\chi}} \mathfrak{e}_{u}$ from (51), and note that the longitudinal component of the second term vanishes because of I.5.3.1 (2c). Thus,

$$
\int_{\gamma_{u_{j-1}}^{-1}(0)}^{\gamma_{u_{j}}^{-1}(0)} \ell^{-1} \frac{E_{w_{\chi}} \mathfrak{e}_{u} L}{=} \int_{\gamma_{u_{j-1}}^{-1}(0)}^{\gamma_{u_{j}}^{-1}(0)} \ell^{-1}\left(\gamma_{u}^{\prime}-1\right) \underline{u_{\gamma \gamma}\left(\gamma_{u}\right)} L .
$$

To estimate the integral on the RHS, note that on the interval of integration, $\gamma_{u_{j}}, \gamma_{u_{j-1}}$ are negative/positive, respectively. Also,

$$
\begin{cases}\left|\ell^{-1}\left(\gamma_{u}^{\prime}-1\right) \underline{u}_{\gamma \gamma}\right| \leq C_{1} \lambda & \text { when }\left|\gamma_{u}\right| \leq \gamma_{0} \\ C_{2}^{\prime} \lambda\left|\gamma_{u}\right| \leq\left|\ell^{-1}\left(\gamma_{u}^{\prime}-1\right) \underline{u_{\gamma \gamma}}{ }_{L}\right| \leq C_{2} \lambda\left|\gamma_{u}\right| & \text { when } \gamma_{0} \leq\left|\gamma_{u}\right| \leq \epsilon^{1 / 2} \lambda^{-1 / 2} \\ C_{3}^{\prime}\left|\gamma_{u}\right|^{-1} \leq\left|\ell^{-1}\left(\gamma_{u}^{\prime}-1\right) \underline{u_{\gamma \gamma}}{ }_{L}\right| \leq C_{3}\left|\gamma_{u}\right|^{-1} & \text { when }\left|\gamma_{u}\right| \geq \epsilon^{1 / 2} \lambda^{-1 / 2}\end{cases}
$$

Furthermore, when $\left|\gamma_{u}\right| \geq \gamma_{0}$, the sign of $\ell^{-1}\left(\gamma_{u}^{\prime}-1\right) \underline{u_{\gamma \gamma}} L$ is the sign of $\gamma_{u}$. Thus, the contribution to the first integral from the two regions where $\left|\gamma_{u}\right| \geq \gamma_{0}$ is bounded above and below by expressions of the form

$$
\operatorname{sign}\left(\gamma_{u}\right) C_{2}^{\prime \prime}\left(\int_{\gamma_{0}}^{\epsilon^{1 / 2} \lambda^{-1 / 2}} \lambda \gamma\left(\gamma^{\prime}\right)^{-1} d \gamma+\int_{\epsilon^{1 / 2} \lambda^{-1 / 2}}^{\infty} \gamma^{-1}\left(\gamma^{\prime}\right)^{-1} d \gamma\right)
$$

By our estimate for $\gamma^{\prime}$ in Section 2, this is in turn bounded above and below by $\operatorname{sign}\left(\gamma_{u}\right) C$, where $C>0$ is a $\lambda$-independent constant, and the sign is - when $u=u_{j}$; + when $u=u_{j-1}$. Meanwhile, the contribution from the region where $\left|\gamma_{u}\right| \leq \gamma_{0}$ is bounded by $C^{\prime} \lambda$; therefore ignorable. In sum, we have the claimed estimate, and hence the assertion (b).

(c) Let $\eta=\hat{n}_{\left(\lambda, w_{\chi}\right)}\left(\alpha, \xi+\sum_{i=0}^{k+1} \varphi_{i} \mathfrak{e}_{u_{i}}\right)-\hat{n}_{\left(\lambda, w_{\chi}\right)}\left(\alpha^{\prime}, \xi^{\prime}+\sum_{i=0}^{k+1} \varphi_{i}^{\prime} \mathfrak{e}_{u_{i}}\right)$ in (41). The second term in it, by (54) and (49), is bounded by $C \lambda^{1 /(2 q)} \lambda^{1 /(2 q)}\left|\hat{\varphi}-\hat{\varphi}^{\prime}\right|$. On the other hand, by Hölder inequality and the same direct computation that appeared in the end of the proof of last lemma, the first term of (41) 
can be bounded in absolute value by writing $I_{j}=\left(\gamma_{u_{j-1}}^{-1}(0), \gamma_{u_{j}}^{-1}(0)\right)$

$$
\begin{aligned}
& C \lambda^{1 /(2 q)}\left(\sum _ { i } | \varphi _ { i } - \varphi _ { i } ^ { \prime } | \left(\lambda\left(\left|\varphi_{i}\right|+\left|\varphi_{i}^{\prime}\right|\right) \int_{I_{j}} \ell^{-1}\left\|\left(u_{i}\right)_{\gamma}\right\|_{2, t}^{2} d s\right.\right. \\
& \left.+\left(|\alpha|+\left|\alpha^{\prime}\right|\right) \int_{I_{j}} \ell^{-1} \underline{\left(u_{i}\right)_{\gamma}} L d s\right) \\
& +\left(\|\xi\|_{W_{\chi}}+\left\|\xi^{\prime}\right\|_{W_{\chi}}\right)\left(\left\|\xi-\xi^{\prime}\right\|_{W_{\chi}} \lambda^{-1 / 2+1 / p}\right. \\
& \left.\quad+\sum_{i}\left|\varphi_{i}-\varphi_{i}^{\prime}\right|\left\|\ell^{-1 / 2}\left(u_{i}\right)_{\gamma}\right\|_{L^{q}\left(I_{j} \times S^{1}\right)}+\left|\alpha-\alpha^{\prime}\right|\left\|\ell^{-1 / 2}\right\|_{L^{q}\left(I_{j}\right)}\right) \\
& +\left|\alpha-\alpha^{\prime}\right|\left(\left(|\alpha|+\left|\alpha^{\prime}\right|\right) \int_{I_{j}} \ell^{-1} d s+\sum_{i}\left(\left|\varphi_{i}\right|+\left|\varphi_{i}^{\prime}\right|\right) \int_{I_{j}} \ell^{-1}\left(u_{i}\right)_{\gamma} d s\right) \\
& +\left\|\xi-\xi^{\prime}\right\|_{W_{\chi}}\left(\sum_{i}\left(\left|\varphi_{i}\right|+\left|\varphi_{i}^{\prime}\right|\right)\left\|\ell^{-1 / 2}\left(u_{i}\right)_{\gamma}\right\|_{L^{q}\left(I_{j} \times S^{1}\right)}\right. \\
& \left.\left.\quad+\left(|\alpha|+\left|\alpha^{\prime}\right|\right)\left\|\ell^{-1 / 2}\right\|_{L^{q}\left(I_{j}\right)}\right)\right) \\
& \leq C^{\prime} \lambda^{1 /(2 q)}\left(\lambda^{1 /(2 q)}\left|\hat{\varphi}-\hat{\varphi}^{\prime}\right|+\left(\varepsilon_{1}+C^{\prime \prime} \lambda^{1 /(2 p)}\right)\left\|\xi-\xi^{\prime}\right\|_{W_{\chi}}\right) \\
& \leq C_{3} \lambda^{1 / q}\left|\hat{\varphi}-\hat{\varphi}^{\prime}\right| .
\end{aligned}
$$

In the above we again used (49), the estimate for $\ell$ in Lemma 3.3.2, and the estimates for $\gamma_{u_{i}}$ and $\sigma_{\chi}$ in section 2. Summing up, this gives us assertion (c).

4.1.4. Constructing the gluing map. It follows immediately from the previous lemma that the linearization of the Kuranishi map is surjective, and hence the moduli space is (Zariski) smooth. More precisely, choose

$$
Q_{\chi}:=\operatorname{Span}\left\{\mathfrak{e}_{u_{1}}, \ldots, \mathfrak{e}_{u_{k+1}}\right\} \subset K_{\chi} \quad Q^{\Xi}:=\bigcup_{\chi} Q_{\chi}
$$

The reductions of the K-models $\left[K_{\chi} \rightarrow C_{\chi}\right]_{W_{\chi}^{\prime}},\left[\hat{K}_{\chi} \rightarrow C_{\chi}\right]_{W_{\chi}^{\prime}}$ by $Q_{\chi}$ give respectively the standard K-models for $E_{w_{\chi}}$ and $\hat{E}_{\left(\lambda, w_{\chi}\right)}$ :

$$
\left[\operatorname{ker} E_{w_{\chi}} \rightarrow *\right]_{Q_{\chi} \oplus W_{\chi}^{\prime}}, \quad\left[\operatorname{ker} \hat{E}_{\left(\lambda, w_{\chi}\right)} \rightarrow *\right]_{Q_{\chi} \oplus W_{\chi}^{\prime}} .
$$

Indeed, from Lemma 4.1.3 (b), we see that the $(k+1) \times(k+1)$-matrix $E=\left(E_{j i}\right)$,

$$
E_{j i}:=\lambda^{-1 /(2 q)} P_{\mathfrak{f}_{j}}\left(E_{w_{\chi}} \mathfrak{e}_{u_{i}}\right), \quad i, j \in\{1, \ldots, k+1\}
$$


is, up to ignorable terms, of the form

$$
\left(\begin{array}{ccccc}
- & 0 & \cdots & \cdots & 0 \\
+ & - & 0 & \cdots & 0 \\
0 & + & \ddots & \cdots & 0 \\
\vdots & 0 & \ddots & \ddots & \vdots \\
0 & \cdots & \cdots & + & -
\end{array}\right) \quad(+/- \text { denote positive/negative numbers of } O(1))
$$

Thus, it has a uniformly bounded inverse, denoted $\left(G_{i j}\right)$. Restricted to $Q_{\chi}$ (i.e., setting $\alpha=\varphi_{0}=0$ ), (47) can be rewritten in the form

$$
\vec{\varphi}=\Psi(\vec{\varphi}), \quad \text { where } \vec{\varphi}:=\left(\varphi_{1}, \ldots, \varphi_{k+1}\right),
$$

and $\Psi: \mathbb{R}^{k+1} \rightarrow \mathbb{R}^{k+1}$ is the map given by

$$
(\Psi(\vec{\varphi}))_{i}=-\sum_{j=1}^{k+1} G_{i j} \lambda^{-1 /(2 q)} P_{\mathfrak{f}_{j}}\left(\bar{\partial}_{J X_{\lambda}} w_{\chi}+n_{w_{\chi}}\left(\xi(\vec{\varphi})+\sum_{l=1}^{k+1} \varphi_{l} \mathfrak{e}_{u_{l}}\right)\right) .
$$

Note from the uniform boundedness of $\left(G_{i j}\right)$ and Lemmas 4.1.2, 4.1.3 (a) that

$$
\begin{aligned}
|\Psi(\overrightarrow{0})| & \leq \sum_{j} C \lambda^{-1 /(2 q)}\left(\left|P_{\mathfrak{f}_{j}} \bar{\partial}_{J X_{\lambda}} w_{\chi}\right|+\left\|n_{w_{\chi}}(\xi(\overrightarrow{0}))\right\|_{L_{\chi}}\right) \\
& \leq C_{2} \lambda^{-1 /(2 q)}\left(\lambda^{1 / q}+\|\xi(\overrightarrow{0})\|_{W_{\chi}}^{2}\right) \\
& \leq C_{2}^{\prime} \lambda^{1 /(2 q)} \ll 1 .
\end{aligned}
$$

On the other hand, Lemmas 4.1.2 and 4.1.3 (c) and the uniform boundedness of $\left(G_{i j}\right)$ again imply:

$\left|\Psi(\vec{\varphi})-\Psi\left(\overrightarrow{\varphi^{\prime}}\right)\right| \leq K\left|\vec{\varphi}-\overrightarrow{\varphi^{\prime}}\right|$ for a positive constant $K \leq C \lambda^{1 /(2 q)} \ll 1$.

Thus by the contraction mapping theorem, we have a unique solution of (55) among all small enough $\vec{\varphi}$.

To summarize, for sufficiently small $\lambda_{0}>0$, there is a universal positive constant $C_{w}$, such that for all $\chi \in \Xi(\mathbb{S})$, there is a unique

$$
\left(\vec{\varphi}_{\chi}, \xi_{\chi}\right) \in Q_{\chi} \oplus W_{\chi}^{\prime} \quad \text { with }\left\|\xi_{\chi}\right\|_{W_{\chi}}^{2}+\left|\vec{\varphi}_{\chi}\right|^{2} \leq C_{w}
$$

which solves (46), (47). In fact, the solution satisfies

$$
\left\|\xi_{\chi}+\sum_{i} \varphi_{\chi, i} \mathfrak{e}_{u_{i}}\right\|_{W_{\chi}}^{2} \leq\left\|\xi_{\chi}\right\|_{W_{\chi}}^{2}+C^{\prime}\left|\vec{\varphi}_{\chi}\right|^{2} \leq C \lambda^{1 / q}
$$

because of (56) and (48).

We define the gluing map to be the map from $\Xi(\mathbb{S})$ to $\hat{\mathcal{M}}_{P}^{\Lambda}$ sending

$$
\chi \mapsto \exp \left(w_{\chi}, \xi_{\chi}+\sum_{i=1}^{k+1} \varphi_{\chi, i} \mathfrak{e}_{u_{i}}\right) .
$$


4.2. Surjectivity of the gluing map. With the gluing map constructed above, the standard arguments outlined in Section 1.2.7 shows that it is a local diffeomorphism onto $\hat{\mathcal{M}}_{P}^{\Lambda}(\mathbf{x}, \mathbf{z}) \cap U_{\varepsilon}$, the intersection of the moduli space with a tubular neighborhood $U_{\varepsilon}$ of the image of the pregluing map in $\mathcal{B}$-topology.

As our goal is instead to show that the gluing map is a local diffeomorphism onto (the interior of) a neighborhood of $\mathbb{S} \subset \hat{\mathcal{M}}_{P}^{\Lambda,+}(\mathbf{x}, \mathbf{z})$ in the coarser chain topology, we need to show that the latter neighborhood in fact lies in $U_{\varepsilon}$. This is done via the following variant of decay estimates for flows near $y$.

4.2.1. $\boldsymbol{w}$ in terms of $\tilde{w}$ and $\tilde{\xi}$. Let $(\lambda, \hat{w}) \in \hat{\mathcal{M}}_{P}^{\left(-\lambda_{0}, \lambda_{0}\right), 1}\left(\mathbf{x}, \mathbf{z} ; \mathrm{wt}_{-\langle y\rangle, e_{\mathcal{P}}} \leq\right.$ $\Re)$ be in a chain-topology neighborhood of $\mathbb{S} \subset \hat{\mathcal{M}}_{P}^{\Lambda,+}(\mathbf{x}, \mathbf{z})$. Namely, $|\lambda| \ll 1$, and there is a broken trajectory $\hat{\chi}:=\left\{u_{0}, u_{1}, \ldots, u_{k+1}\right\} \in \mathbb{S}$, which is close to $\hat{w}$ in chain topology. Let $\chi:=(\hat{\chi}, \lambda) \in \Xi(\mathbb{S})$. We may find a representative $w$ of $\hat{w}$, such that

$$
w(s)=\exp (\tilde{w}(s), \tilde{\xi}(s)),
$$

where $w, \tilde{w}$ are chosen such that

- $\tilde{w}(s)=y$ at $s=\tilde{\mathfrak{l}}_{1}, \ldots, \tilde{\mathfrak{l}}_{k+1} ; \tilde{\mathfrak{l}}_{1}<\tilde{\mathfrak{l}}_{2}<\cdots<\tilde{\mathfrak{l}}_{k+1}$ subdivide $\mathbb{R}$ into $k+2$ open intervals $I_{i}, i=0,1, \ldots, k+1$;

- $\tilde{w}(s)=u_{i}\left(\tilde{\gamma}_{u_{i}}(s)\right)$ over $I_{i}$, where $\tilde{\gamma}_{u_{i}}: I_{i} \rightarrow \mathbb{R}$ are homeomorphisms determined by:

$$
\begin{cases}\Pi_{\mathbf{e}_{y}} \tilde{\zeta}(s)=\Pi_{\mathbf{e}_{y}} \zeta(s) & \text { for } s \in \bigcup_{i}\left[\tilde{\gamma}_{u_{i-1}}^{-1}\left(\gamma_{0}\right), \tilde{\gamma}_{u_{i}}^{-1}\left(-\gamma_{0}\right)\right], \\ \tilde{\gamma}_{u_{i}}^{\prime}(s)=1 & \text { for } s \in \mathbb{R} \backslash \bigcup_{i}\left[\tilde{\gamma}_{u_{i-1}}^{-1}\left(\gamma_{0}\right), \tilde{\gamma}_{u_{i}}^{-1}\left(-\gamma_{0}\right)\right],\end{cases}
$$

with $\zeta, \tilde{\zeta}$ given by $\tilde{w}(s)=\exp (y, \tilde{\zeta}(s)), w(s)=\exp (y, \zeta(s))$, and $\gamma_{0}$ the large positive constant in Section 2.4.

$$
\gamma_{u_{0}}^{-1}(0)=\tilde{\gamma}_{u_{0}}^{-1}(0) ; \quad\left\langle\left(u_{0}\right)_{\gamma}(0), \tilde{\gamma}_{u_{0}}^{*} \tilde{\xi}(0)\right\rangle_{2, t}=0 .
$$

Because of elliptic regularity and the fact that $(\lambda, w)$ is close to $\mathbb{S}$ in chain topology, we may assume without loss of generality that

$$
\begin{gathered}
\|\tilde{\xi}(s)\|_{\infty, 1, t}+\left\|\tilde{\xi}^{\prime}(s)\right\|_{\infty, 1, t}<\varepsilon \forall s \quad \text { for }|\lambda|^{1 / 2}<\varepsilon \ll 1 ; \\
\tilde{\gamma}_{u_{j}}^{-1}(0)-\tilde{\gamma}_{u_{j-1}}^{-1}(0)>\varepsilon^{-1} \quad \forall j \in\{1, \ldots, k+1\} .
\end{gathered}
$$

4.2.2. Estimating $\tilde{\xi}$. Because of the large weights near $y$, we need the following more refined pointwise estimate for $\tilde{\xi}$ near $y$.

Lemma. Let $(\lambda, w) \in \mathcal{M}_{P}^{\Lambda}$ be close to $\mathbb{S}$ in chain topology, and let $y$ be a death as before. Then $\lambda>0$. Furthermore, in the notation of Section 4.2.1, there is a small positive constant $\varepsilon_{0}=\varepsilon_{0}\left(\gamma_{0}^{-1}, \varepsilon\right)$ independent of $w$, such that

$$
\|\tilde{\xi}(s)\|_{2,2, t}+\left\|\tilde{\xi}^{\prime}(s)\right\|_{2,1, t} \leq \varepsilon_{0}\left(\left\|\Pi_{\mathbf{e}_{y}} \tilde{\zeta}(s)\right\|_{2,1, t}^{4}+|\lambda|\right)
$$

for all $s \in \bigcup_{j}\left[\tilde{\gamma}_{u_{j-1}}^{-1}\left(\gamma_{0}\right), \tilde{\gamma}_{u_{j}}^{-1}\left(-\gamma_{0}\right)\right]$. 
Proof. Let $s \in \bigcup_{j}\left[\tilde{\gamma}_{u_{j-1}}^{-1}\left(\gamma_{0}\right), \tilde{\gamma}_{u_{j}}^{-1}\left(-\gamma_{0}\right)\right]$ throughout this proof. In fact, it suffices to consider only one $j$.

To estimate $\xi$, it is equivalent to estimate $\zeta-\tilde{\zeta}$, which we denote by $c$. The assumption (57) implies that $c \in \operatorname{ker} A_{y}^{\perp}$.

Write $b:=\Pi_{\operatorname{ker} A_{y}} \tilde{\zeta}$, and let $Z$ : $\operatorname{ker} A_{y} \rightarrow \operatorname{ker} A_{y}^{\perp}$ be such that $\tilde{\zeta}=$ $(1+Z) b$. Similar to I.(38), I.(39), the flow equation can be rewritten as:

$$
\begin{aligned}
-\frac{d \tilde{\zeta}}{d s}= & \left(1+\nabla_{b} Z\right)\left(\lambda C_{y}^{\prime} \mathbf{e}_{y}+\Pi_{\mathrm{ker} A_{y}} \hat{n}_{(0, y)}(\lambda, \tilde{\zeta}+c)\right) \\
-\frac{d c}{d s}=A_{y} c- & \nabla_{b} Z\left(\lambda C_{y}^{\prime} \mathbf{e}_{y}\right) \\
& +\left(1-\Pi_{\mathrm{ker} A_{y}}-\nabla_{b} Z \Pi_{\mathrm{ker} A_{y}}\right)\left(\hat{n}_{(0, y)}(\lambda, \tilde{\zeta}+c)-n_{y}(\tilde{\zeta})\right) .
\end{aligned}
$$

Taking the $L_{t}^{2}$-inner product of $(61)$ with $c$ and rearranging like the proof of Sublemma I.5.1.7, we get (adopting the notation of I.5.1)

$$
\begin{gathered}
\frac{d\left\|c_{+}\right\|_{2, t}}{d s} \geq \mu_{+}\left\|c_{+}\right\|_{2, t}-\epsilon_{+}\|c\|_{2, t}-C_{+}|\lambda|\|\tilde{\zeta}\|_{2,1, t}, \\
\frac{d\left\|c_{-}\right\|_{2, t}}{d s} \leq-\mu_{-}\left\|c_{-}\right\|_{2, t}+\epsilon_{-}\|c\|_{2, t}+C_{-}|\lambda|\|\tilde{\zeta}\|_{2,1, t}
\end{gathered}
$$

Subtracting a suitable multiple of the first inequality from the second, one obtains:

$$
\left(\left\|c_{-}\right\|_{2, t}-\epsilon_{-}^{\prime}\left\|c_{+}\right\|_{2, t}\right)^{\prime} \leq-\mu_{-}^{\prime}\left\|c_{-}\right\|_{2, t}+C_{-}^{\prime} \gamma_{0}^{-1}|\lambda| .
$$

Taking convolution product with the integral kernel of $d / d s+\mu_{-}^{\prime}$ on both sides, one gets

$$
\left\|c_{-}\right\|_{2, t}-\epsilon_{-}^{\prime}\left\|c_{+}\right\|_{2, t} \leq C_{-} \varepsilon \mathrm{e}^{-\mu^{\prime}\left(s-\tilde{\gamma}_{u_{-1}}^{-1}\left(\gamma_{0}\right)\right)}+C_{-}^{\prime \prime} \gamma_{0}^{-1}|\lambda|,
$$

and similarly,

$$
\left\|c_{+}\right\|_{2, t}-\epsilon_{+}^{\prime}\left\|c_{-}\right\|_{2, t} \leq C_{+} \varepsilon \mathrm{e}^{-\mu^{\prime}\left(\tilde{\gamma}_{u_{j}}^{-1}\left(-\gamma_{0}\right)-s\right)}+C_{+}^{\prime \prime} \gamma_{0}^{-1}|\lambda| .
$$

Adding the above two inequalities, we get

$$
\|c\|_{2, t} \leq C \varepsilon\left(\mathrm{e}^{-\mu^{\prime}\left(s-\tilde{\gamma}_{u_{j-1}}^{-1}\left(\gamma_{0}\right)\right)}+\mathrm{e}^{-\mu^{\prime}\left(\tilde{\gamma}_{u_{j}}^{-1}\left(-\gamma_{0}\right)-s\right)}\right)+C^{\prime \prime} \gamma_{0}^{-1}|\lambda| .
$$

This may be improved to give a similar estimate for $\|c\|_{2,1, t}$ using (61) by the same elliptic bootstrapping and Sobolev embedding argument as in I.5.1.7.

On the other hand, write $b(s)=\underline{b}(s) \mathbf{e}_{y}$ as usual, and notice that by taking $\Pi_{\text {ker } A_{y}}$ of $(60), b(s)$ satisfies:

$$
-b^{\prime}(s)=\lambda C_{y}^{\prime} \mathbf{e}_{y}+\Pi_{\text {ker } A_{y}} \hat{n}_{(0, y)}(\lambda, \tilde{\zeta}+c) .
$$

Integrating this equation, it is easy to see that $\lambda<0$ would contradict the fact that, due to the proximity of $w$ and $\tilde{w}$,

$$
\underline{b}\left(\tilde{\gamma}_{u_{j-1}}^{-1}\left(\gamma_{0}\right)\right)>0, \quad \underline{b}\left(\tilde{\gamma}_{u_{j}}^{-1}\left(-\gamma_{0}\right)\right)<0, \quad \tilde{\gamma}_{u_{j-1}}^{-1}\left(\gamma_{0}\right)<\tilde{\gamma}_{u_{j}}^{-1}\left(-\gamma_{0}\right) .
$$

Thus, $\lambda$ must be positive. 
On the other hand, as $\lambda>0,(62)$ implies that $\underline{b}(s)$ decreases monotonically with $s$. We now claim that

$$
\mathrm{e}^{-\mu^{\prime}\left(s-\tilde{\gamma}_{u_{j-1}}^{-1}\left(\gamma_{0}\right)\right)}+\mathrm{e}^{-\mu^{\prime}\left(\tilde{\gamma}_{u_{j}}^{-1}\left(-\gamma_{0}\right)-s\right)} \leq C_{e}\left(\underline{b}(s)^{4}+|\lambda|\right) .
$$

Combined with the above estimates for $c$, this would then imply the second assertion of the lemma.

To prove the claim, note that by symmetry and the decay/growth behavior of the two terms on the LHS, it suffices to show that

$$
\begin{gathered}
\underline{b}^{4}(s) \geq C_{1} \mathrm{e}^{-\mu^{\prime}\left(\tilde{\gamma}_{u_{j}}^{-1}\left(-\gamma_{0}\right)-s\right)} \quad \text { when }-1 \ll \underline{b}(s) \leq-|\lambda|^{1 / 2} ; \\
\underline{b}^{4}(s) \geq C_{2} \mathrm{e}^{-\mu^{\prime}\left(s-\tilde{\gamma}_{u_{j-1}}^{-1}\left(\gamma_{0}\right)\right)} \quad \text { when } 1 \gg \underline{b}(s) \geq|\lambda|^{1 / 2}
\end{gathered}
$$

for $s$-independent constants $C_{1}, C_{2}$. We shall only demonstrate the second inequality since the first is similar. When $\underline{b}(s) \geq|\lambda|^{1 / 2},(62)$ together with the above estimate for $\|c\|_{2,1, t}$ imply that

$$
\left(\underline{b}^{4}\right)^{\prime} \geq-\mu^{\prime} \underline{b}^{4}-C_{b} \varepsilon^{4} \mathrm{e}^{-4 \mu^{\prime}\left(s-\tilde{\gamma}_{u_{j-1}}^{-1}\left(\gamma_{0}\right)\right)} .
$$

Taking convolution product with the integral kernel of $d / d s+\mu^{\prime}$, we get in this region

$$
\underline{b}^{4}(s) \geq C_{6} \mathrm{e}^{-\mu^{\prime}\left(s-\tilde{\gamma}_{u_{j-1}}^{-1}\left(\gamma_{0}\right)\right)}-C_{7} \varepsilon^{4} \mathrm{e}^{-4 \mu^{\prime}\left(s-\tilde{\gamma}_{u_{j-1}}^{-1}\left(\gamma_{0}\right)\right)},
$$

and hence the claim.

4.2.3. From $\tilde{w}$ to $w_{\chi}$. Next, notice that $\tilde{w}$ differs from the pregluing $w_{\chi}$ by a reparameterization. We shall estimate the difference between $\tilde{w}$ and $w_{\chi}$ by estimating the difference between $\tilde{\gamma}_{u_{i}}^{-1}$ and $\gamma_{u_{i}}^{-1}$.

Similar to $\gamma_{u_{i}}$ (see Section 2.2.1), $\tilde{\gamma}_{u_{i}}$ satisfies:

$$
\left\langle u_{\gamma}\left(\tilde{\gamma}_{u_{i}}\right),-\left(\tilde{\gamma}_{u_{i}}^{\prime}-1\right) u_{\gamma}\left(\tilde{\gamma}_{u_{i}}\right)+\lambda Y_{(0, \tilde{w})}+E_{\tilde{w}} \tilde{\xi}+\tilde{n}(\lambda, \tilde{\xi})\right\rangle_{2, t}=0,
$$

where $\tilde{n}$ is some nonlinear term in $\lambda, \tilde{\xi}$. By (57), when $\tilde{\gamma}_{u_{i}}(s) \geq\left|\gamma_{0}\right|$,

$$
\begin{aligned}
& \left\|u_{\gamma}\left(\tilde{\gamma}_{u_{i}}\right)\right\|_{2, t}^{-1}\left|\left\langle u_{\gamma}\left(\tilde{\gamma}_{u_{i}}\right), E_{\tilde{w}} \tilde{\xi}+\tilde{n}(\lambda, \tilde{\xi})\right\rangle_{2, t}\right| \\
& \quad \leq C_{8}\left(\|\tilde{\xi}\|_{2,1, t}+\left\|\tilde{\xi}^{\prime}\right\|_{2, t}\right) / \tilde{\gamma}_{u_{i}}+C_{8}^{\prime}\left(\|\tilde{\xi}\|_{2,1, t}+\lambda\right)^{2} .
\end{aligned}
$$

Write $\gamma_{\lambda, u_{i}}=\gamma_{u_{i}}$ in (11) to emphasize the parameter $\lambda$ used in the definition, and let

$$
\Delta_{s, i}(\gamma):=\gamma_{\lambda, u_{i}}^{-1}(\gamma)-\tilde{\gamma}_{u_{i}}^{-1}(\gamma)
$$

Comparing the defining equations for $\gamma_{\lambda, u_{i}}$ and $\tilde{\gamma}_{\lambda, u_{i}}$, and using (59) and the above estimate for $\left\|u_{\gamma}\left(\tilde{\gamma}_{u_{i}}\right)\right\|_{2, t}^{-1}\left|\left\langle u_{\gamma}\left(\tilde{\gamma}_{u_{i}}\right), E_{\tilde{w}} \tilde{\xi}+\tilde{n}(\lambda, \tilde{\xi})\right\rangle_{2, t}\right|$, we find that for 
all $i \in\{0, \ldots, k\}$,

$$
\begin{aligned}
& \left|\Delta_{s, i}\left(\gamma_{+}\right)-\Delta_{s, i}\left(\gamma_{-}\right)\right| \leq \\
& \begin{cases}\left|\int_{0}^{\gamma_{0}}(O(\varepsilon)+O(\lambda)) d \gamma\right| \leq C_{1} \varepsilon & \text { if } 0 \leq \gamma_{+}, \gamma_{-} \leq \gamma_{0} ; \\
\left|\int_{\gamma_{0}}^{\mathfrak{r}_{i}} C_{7}\left(\varepsilon \gamma\left(\lambda+\gamma^{-4}\right)+\left(\lambda+\gamma^{-4}\right)^{2} \gamma^{2}\right) d \gamma\right| \leq C_{6} \varepsilon & \text { if } \gamma_{0}<\gamma_{+}, \gamma_{-} \leq \mathfrak{r}_{i} ; \\
\left|\int_{\mathfrak{r}_{i}}^{\infty} \frac{C_{5}\left(\varepsilon \gamma\left(\lambda+\gamma^{-4}\right)+\left(\lambda+\gamma^{-4}\right)^{2} \gamma^{2}\right) d \gamma}{\left(1+\lambda \gamma^{2}\right)^{2}}\right| \leq C_{4} \varepsilon & \text { if } \gamma_{+}, \gamma_{-} \geq \mathfrak{r}_{i} .\end{cases}
\end{aligned}
$$

Similar estimates hold for negative $\gamma_{+}, \gamma_{-}$when $i \in\{1, \ldots, k+1\}$. For $i=0$ and any two negative $\gamma_{+}, \gamma_{-}$, or for $i=k+1$ and any two positive $\gamma_{+}, \gamma_{-}$, the estimate in the first case above holds.

Combining this with the initial conditions from (58):

$$
\Delta_{s, 0}(0)=0 ; \quad \Delta_{s, i-1}(\infty)=\Delta_{s, i}(-\infty) \forall i \in\{1, \ldots, k+1\},
$$

we have

$$
\left|\Delta_{s, i}(\gamma)\right| \leq C \varepsilon \forall \gamma, i \text { for a } \lambda \text {-independent constant } C>0 .
$$

Applying the mean value theorem and the estimate for $w_{\chi}^{\prime}$ in (20), and recalling the assumption (58), we see that $\tilde{w}=\exp \left(w_{\chi}, \tilde{\xi}_{\chi}\right)$ for an $\tilde{\xi}_{\chi}$ with:

$$
\begin{gathered}
\left\langle\left(u_{0}\right)_{\gamma},(0),\left(\gamma_{u_{0}}^{-1}\right)^{*} \tilde{\xi}_{\chi}(0)\right\rangle_{2, t}=0 \\
\left\|\tilde{\xi}_{\chi}\right\|_{2,1, t} \leq C^{\prime}\left(\lambda+\varepsilon \gamma_{u_{i}}^{-2}\right) \quad \text { on } I_{i} \cap \bigcup_{j}\left[\gamma_{u_{j-1}}^{-1}\left(\gamma_{0}\right), \gamma_{u_{j}}^{-1}\left(-\gamma_{0}\right)\right] \forall i
\end{gathered}
$$

4.2.4. From pointwise estimates to $\hat{W}_{\chi}$ estimates. Recall that our goal is to show that given $(\lambda, \hat{w}) \in \hat{\mathcal{M}}_{P}^{\Lambda}$ in a chain-topology neighborhood of $\mathbb{S}$, as prescribed in Section 4.2.1, we may write

$$
(\lambda, w)=e\left(\lambda^{\prime}, w_{\chi^{\prime}} ; \alpha_{\chi^{\prime}}, \xi_{\chi^{\prime}}\right)
$$

for some $\chi^{\prime}=\left(\underline{\chi}, \lambda^{\prime}\right) \in \Xi(\mathbb{S}), \underline{\chi}:=\left\{\hat{u}_{0}, \ldots, \hat{u}_{k+1}\right\}$, with $\left(\alpha_{\chi^{\prime}}, \xi_{\chi^{\prime}}\right)$ satisfying

$$
\text { (1) }\left\|\left(\alpha_{\chi^{\prime}}, \xi_{\chi^{\prime}}\right)\right\|_{\hat{W}_{\chi^{\prime}}} \leq C \varepsilon ; \quad(2)\left(\alpha_{\chi^{\prime}}, \xi_{\chi^{\prime}}\right) \in B_{\chi^{\prime}},
$$

where $C$ is a $\lambda$-independent constant, and $B_{\chi^{\prime}}$ is the B-space chosen so that $\left[\text { ker } \hat{E}_{\left(\lambda^{\prime}, w_{\chi^{\prime}}\right)} \rightarrow *\right]_{B_{\chi^{\prime}}}$ forms a K-model for $\hat{E}_{\left(\lambda^{\prime}, w_{\chi^{\prime}}\right)}$.

Lemma. Suppose the $\left(\alpha_{\chi^{\prime}}, \xi_{\chi^{\prime}}\right)$ given in (65) satisfies

$$
\begin{aligned}
\left|\alpha_{\chi^{\prime}}\right| & \leq C^{\prime} \lambda^{3 / 2} \varepsilon ; \\
\left\|\xi_{\chi^{\prime}}\right\|_{2,1, t} & \leq C_{\xi}^{\prime}\left(\lambda+\varepsilon \gamma_{u_{i}}^{-2}\right) \quad \text { on } I_{i} \cap \bigcup_{j}\left[\gamma_{u_{j-1}}^{-1}\left(\gamma_{0}\right), \gamma_{u_{j}}^{-1}\left(-\gamma_{0}\right)\right] \forall i
\end{aligned}
$$

for $\lambda$-independent constants $C^{\prime}, C_{\xi}^{\prime}$. Then (66.1) holds. 
Proof. First, notice that the assumption on $\alpha_{\chi^{\prime}}$ implies $\left\|\left(\alpha_{\chi^{\prime}}, 0\right)\right\|_{\hat{W}_{\chi^{\prime}}} \leq$ $C_{1} \lambda^{1 / 2-1 /(2 p)} \varepsilon$. On the other hand, the assumption that $(\lambda, \hat{w})$ is close to $\chi^{\prime}$ in chain topology implies that over $\Theta^{c}:=\Theta \backslash \bigcup_{j}\left[\gamma_{u_{j-1}}^{-1}\left(\gamma_{0}\right), \gamma_{u_{j}}^{-1}\left(-\gamma_{0}\right)\right] \times S^{1}$,

$\left\|\xi_{\chi^{\prime}}\right\|_{W_{\chi^{\prime}}\left(\Theta^{c}\right)} \leq C_{2}\left\|\xi_{\chi^{\prime}}\right\|_{L_{1}^{p}\left(\Theta^{c}\right)} \leq C_{3} \varepsilon$ for $\lambda$-independent constants $C_{2}, C_{3}$.

Thus, it remains to estimate $\left\|\xi_{\chi^{\prime}}\right\|_{W_{\chi^{\prime}}\left(\left[\gamma_{u_{j-1}}^{-1}\left(\gamma_{0}\right), \gamma_{u_{j}}^{-1}\left(-\gamma_{0}\right)\right] \times S^{1}\right)}$. We shall focus on estimates on the region $\left[\gamma_{u_{i}}^{-1}\left(\gamma_{0}\right), \gamma_{u_{i}}^{-1}(\infty)\right) \times S^{1}$ for an $i \in\{0, \ldots, k\}$, since estimates on the rest are similar.

By the definition of $\gamma_{u_{i}}$, on this region the flow equation has the form:

$$
\left(\bar{\partial}_{J X_{\lambda^{\prime}}} w_{\chi^{\prime}}\right)_{T_{w}}+\hat{E}_{\left(\lambda^{\prime}, w_{\chi^{\prime}}\right)}\left(\alpha_{\chi^{\prime}}, \xi_{\chi^{\prime}}\right)+\hat{n}_{\left(\lambda^{\prime}, w_{\chi^{\prime}}\right)}\left(\alpha_{\chi^{\prime}}, \xi_{\chi^{\prime}}\right)=0
$$

( $T_{w}$ here means the transverse component with respect to $w_{\chi^{\prime}}^{\prime}$, in contrast to $T_{y}$ below).

Subdivide the region again into $\left[\gamma_{u_{i}}^{-1}\left(\mathfrak{r}_{i}\right), \gamma_{u_{i}}^{-1}(\infty)\right) \times S^{1}$ and the rest.

Over the first region, namely when $\gamma_{u_{i}} \geq \mathfrak{r}_{i}$, in place of $\xi_{\chi^{\prime}}$ and its $W_{\chi^{\prime}}$-norm, it is equivalent to estimate

$$
\begin{gathered}
\xi_{0}:=T_{w_{\chi^{\prime}}, \bar{y}} \xi_{\chi^{\prime}} \in \Gamma\left(\bar{y}^{*} K\right) \quad \text { in the norm } \\
\left\|\xi_{0}\right\|_{W_{0}^{\lambda^{\prime}}}:=\left(\lambda^{\prime}\right)^{-1 / 2}\left\|\xi_{0}\right\|_{p, 1}+\left(\lambda^{\prime}\right)^{-1}\left\|\left(\xi_{0}\right)_{L_{y}}^{\prime}\right\|_{p},
\end{gathered}
$$

where $\left(\xi_{0}\right)_{L_{y}}(s)=\left\langle\mathbf{e}_{y}, \xi_{0}(s)\right\rangle_{2, t} \mathbf{e}_{y}$ is the "longitudinal direction with respect to $y$." Notice that $\mathbf{e}_{y}$ differs from the original longitudinal direction $T_{w_{\chi^{\prime}}(s), y} w_{\chi^{\prime}}^{\prime}(s)\left\|w_{\chi^{\prime}}^{\prime}(s)\right\|_{2, t}^{-1}$ by an ignorable factor of $C\left(\lambda^{\prime} / \epsilon\right)^{1 / 2}$. Let the transversal direction $T_{y}$ and the $L_{0}^{\lambda^{\prime}}$-norm be similarly defined.

Rewriting the flow equation in terms of the above transverse and longitudinal directions, we have:

$$
\begin{array}{r}
E_{\bar{y}}\left(\xi_{0 T_{y}}\right)=-\alpha_{\chi^{\prime}}\left(T_{w_{\chi^{\prime}}, \bar{y}} Y_{\left(\lambda, w_{\chi^{\prime}}\right.}\right)_{T_{y}}+Z_{T_{y}}+\Upsilon_{T_{y}} \\
-\left(T_{w_{\chi^{\prime}}, \bar{y}} \hat{n}_{\left(\lambda^{\prime}, w_{\chi^{\prime}}\right)}\left(\alpha_{\chi^{\prime}}, \xi_{\chi^{\prime}}\right)\right)_{T_{y}}, \\
E_{\bar{y}}\left(\xi_{0 L_{y}}\right)=-\alpha_{\chi^{\prime}}\left(T_{w_{\chi}, \bar{y}} Y_{\left(\lambda, w_{\chi^{\prime}}\right)}\right)_{L_{y}}+Z_{L_{y}}+\Upsilon_{L_{y}} \\
-\left(T_{w_{\chi^{\prime}}, \bar{y}} \hat{n}_{\left(\lambda^{\prime}, w_{\chi^{\prime}}\right)}\left(\alpha_{\chi^{\prime}}, \xi_{\chi^{\prime}}\right)\right)_{L_{y}},
\end{array}
$$

where

- $Z_{T_{y}}, Z_{L_{y}}$ come from the difference between $E_{\bar{y}}$ and $E_{w_{\chi^{\prime}}}$. Thus, their $L_{1, t}^{2}$-norms are bounded by $C\left\|\xi_{\chi^{\prime}}\right\|_{\infty, 1, t} \gamma_{u_{i}}^{-1}$;

- $\Upsilon_{T}, \Upsilon_{L}$ are terms coming from $\left(\bar{\partial}_{J X_{\lambda^{\prime}}} w_{\chi^{\prime}}\right)_{T_{w}}$. The computation in the proof of Lemma 2.4.2 shows that $\left\|\Upsilon_{T_{y}}\right\|_{2,1, t}$ is bounded by $C_{1} \lambda^{\prime} \gamma_{u_{i}}^{-1}$, while $\left\|\Upsilon_{L_{y}}\right\|_{2,1, t}$ is bounded by $C_{2} \lambda^{\prime} \gamma_{u_{i}}^{-2}$. 
Now, the length estimate for $\left[\gamma_{u_{i}}^{-1}\left(\mathfrak{r}_{i}\right), \gamma_{u_{i}}^{-1}(\infty)\right) \times S^{1}$ (cf. (21)) and the assumption (67) yield

$$
\begin{gathered}
\left(\lambda^{\prime}\right)^{-1 / 2}\left(\left\|\xi_{0}\right\|_{L^{p}\left(\left[\gamma_{u_{i}}^{-1}\left(\mathfrak{r}_{i}\right), \gamma_{u_{i}}^{-1}(\infty)\right) \times S^{1}\right)}+\left\|\dot{\xi}_{0}\right\|_{L^{p}\left(\left[\gamma_{u_{i}}^{-1}\left(\mathfrak{r}_{i}\right), \gamma_{u_{i}}^{-1}(\infty)\right) \times S^{1}\right)}\right) \\
\leq C^{\prime}\left(\lambda^{\prime}\right)^{1 / 2-1 /(2 p)} \ll \varepsilon .
\end{gathered}
$$

In addition, the second line of (69) and the above estimates for terms therein, combined with (67) and the length estimate for this region yield

$$
\left(\lambda^{\prime}\right)^{-1}\left\|\xi_{0 L_{y}}^{\prime}\right\|_{L^{p}\left(\left[\gamma_{u_{i}}^{-1}\left(\mathfrak{r}_{i}\right), \gamma_{u_{i}}^{-1}(\infty)\right) \times S^{1}\right)} \leq C_{L}^{\prime}\left(\lambda^{\prime}\right)^{1 / 2-1 /(2 p)} \ll \varepsilon .
$$

In sum, we have

$$
\left\|\xi_{\chi^{\prime}}\right\|_{W_{\chi^{\prime}}\left(\left[\gamma_{u_{i}}^{-1}\left(\mathfrak{r}_{i}\right), \gamma_{u_{i}}^{-1}(\infty)\right) \times S^{1}\right)} \leq C_{1}\left\|\xi_{0}\right\|_{W_{0}^{\lambda^{\prime}}\left(\left[\gamma_{u_{i}}^{-1}\left(\mathfrak{r}_{i}\right), \gamma_{u_{i}}^{-1}(\infty)\right) \times S^{1}\right)} \ll \varepsilon .
$$

To estimate on the second region, namely on $\left[\gamma_{u_{i}}^{-1}\left(\gamma_{0}\right), \gamma_{u_{i}}^{-1}\left(\mathfrak{r}_{i}\right)\right] \times S^{1}$, let $\beta_{i}^{+}(s)$ be a smooth cutoff function with:

- support on $\left[\gamma_{u_{i}}^{-1}\left(\gamma_{0}\right)-1,(1+\epsilon) \gamma_{u_{i}}^{-1}\left(\mathfrak{r}_{i}\right)\right]=: \Theta_{\beta_{i}^{+}}$;

- value 1 over $\left[\gamma_{u_{i}}^{-1}\left(\gamma_{0}\right), \gamma_{u_{i}}^{-1}\left(\mathfrak{r}_{i}\right)\right]$, and

$$
\text { - }\left|\left(\beta_{i}^{+}\right)^{\prime}\right|<C^{\prime} \lambda^{1 / 2} \text { on }\left[\gamma_{u_{1}}^{-1}\left(\mathfrak{r}_{i}\right),(1+\epsilon) \gamma_{u_{i}}^{-1}\left(\mathfrak{r}_{i}\right)\right] \text {. }
$$

Notice that $\left(\gamma_{u_{i}}^{-1}\right)^{*}\left(\beta_{i}^{+} \xi_{\chi^{\prime}}\right) \in W_{u_{i}}^{\prime}$, and since $\left.E_{u_{i}}\right|_{W_{u_{i}}^{\prime}}$ an isomorphism, we have from Lemma 3.2.1 and (68) that

$$
\begin{aligned}
\left\|\beta_{i}^{+} \xi_{\chi^{\prime}}\right\|_{W_{\chi^{\prime}}} \leq\left\|E_{w_{\chi}}\left(\beta_{i}^{+} \xi_{\chi^{\prime}}\right)\right\|_{L_{\chi^{\prime}}} \\
\leq\left|\alpha_{\chi^{\prime}}\right|\left\|\beta_{i}^{+} Y_{\left(\lambda^{\prime}, w_{\chi^{\prime}}\right)}\right\|_{L_{\chi^{\prime}}}+\left\|\beta_{i}^{+}\left(\bar{\partial}_{J X_{\lambda^{\prime}}} w_{\chi^{\prime}}\right)_{T_{w}}\right\|_{L_{\chi^{\prime}}} \\
\quad+\left\|\beta_{i}^{+} \hat{n}_{\left(\lambda^{\prime}, w_{\chi^{\prime}}\right)}\left(\alpha_{\chi^{\prime}}, \xi_{\chi^{\prime}}\right)\right\|_{L_{\chi^{\prime}}}+\left\|\left(\beta_{i}^{+}\right)^{\prime} \xi_{\chi^{\prime}}\right\|_{L_{\chi^{\prime}}} .
\end{aligned}
$$

By assumption (67) and the length estimate $\left|\gamma_{u_{i}}^{-1}\left(\mathfrak{r}_{i}\right)-\gamma_{u_{i}}^{-1}\left(\gamma_{0}\right)\right| \leq C^{\prime}\left(\lambda^{\prime}\right)^{-1 / 2}$, we may bound each terms on the RHS as follows:

- The first term may be bounded by $C_{1}\left|\lambda^{\prime}\right|^{1 / 2-1 /(2 p)}$.

- The second term is already estimated to be small in Proposition 2.4.1.

- The computation in the proof of Lemma 2.5.2 shows that

$$
\begin{aligned}
& \left\|\beta_{i}^{+} \hat{n}_{\left(\lambda^{\prime}, w_{\chi^{\prime}}\right)}\left(\alpha_{\chi^{\prime}}, \xi_{\chi^{\prime}}\right)\right\|_{L_{\chi^{\prime}}} \\
& \quad \leq C_{n}\left(\begin{array}{c}
\left\|\left(\alpha_{\chi^{\prime}}, 0\right)\right\|_{\hat{W}_{\chi^{\prime}}}^{2}+\left\|\left(\alpha_{\chi^{\prime}}, 0\right)\right\|_{\hat{W}_{\chi^{\prime}}} \cdot\left\|\beta_{i}^{+} \xi_{\chi^{\prime}}\right\|_{W_{\chi^{\prime}}} \\
+\left\|\sigma_{\chi^{\prime}}^{1 / 2} \xi_{\chi^{\prime}}\right\|_{L^{\infty}\left(\Theta_{\beta_{i}^{+}}\right)} \cdot\left\|\beta_{i}^{+} \xi_{\chi^{\prime}}\right\|_{W_{\chi^{\prime}}}
\end{array}\right) \\
& \leq C_{n}^{\prime}\left(C_{1}\left(\left|\lambda^{\prime}\right|^{1 / 2-1 /(2 p)}\right)^{2}+C_{2}\left(\left|\lambda^{\prime}\right|^{1 / 2-1 /(2 p)}+\varepsilon \gamma_{0}^{-1}+\lambda^{1 / 2}\right)\left\|\beta_{i}^{+} \xi_{\chi^{\prime}}\right\|_{W_{\chi^{\prime}}}\right) .
\end{aligned}
$$

- By the defining properties of $\beta_{i}^{+}$,

$$
\left\|\left(\beta_{i}^{+}\right)^{\prime} \xi_{\chi^{\prime}}\right\|_{L_{\chi^{\prime}}} \leq C^{\prime \prime}\left|\lambda^{\prime}\right|^{1 / 2-1 /(2 p)} .
$$


Collecting all the above and rearranging, we obtain

$$
\left\|\xi_{\chi^{\prime}}\right\|_{W_{\chi^{\prime}}\left(\left[\gamma_{u_{i}}^{-1}\left(\gamma_{0}\right), \gamma_{u_{i}}^{-1}\left(\mathfrak{r}_{i}\right)\right] \times S^{1}\right)} \leq C_{i} \varepsilon .
$$

Now that we have the estimates for the $W_{\chi^{\prime}}$-norm over all the various regions, we conclude $\left\|\xi_{\chi^{\prime}}\right\|_{W_{\chi^{\prime}}} \leq C \varepsilon$, and hence the claim of the lemma.

4.2.5. Concluding the proof of Proposition 2.1 (a). Recall from Section 4.1.1 the K-model for $\hat{E}_{\left(\lambda^{\prime}, w_{\chi^{\prime}}\right)}$ : $\left[\operatorname{ker} \hat{E}_{\left(\lambda^{\prime}, w_{\chi^{\prime}}\right)} \rightarrow *\right]_{B_{\chi^{\prime}}}$, where $B_{\chi^{\prime}}$ was chosen to be the following subspace of $\hat{W}_{\chi^{\prime}}$ :

$$
B_{\chi^{\prime}}=\left\{\left(0, \xi_{\chi^{\prime}}\right) \mid\left\langle\left(u_{0}\right)_{\gamma}(0),\left(\gamma_{u_{0}}^{-1}\right)^{*} \xi_{\chi^{\prime}}(0)\right\rangle_{2, t}=0\right\} .
$$

Thus, setting $\lambda^{\prime}=\lambda$ and $\chi^{\prime}=\chi,\left(\alpha_{\chi^{\prime}}, \xi_{\chi^{\prime}}\right)=\left(0, \xi_{\chi}\right)$, and $\xi_{\chi}$ can be expressed in terms of $\tilde{\xi}_{\chi}$ (cf. Section 4.2.3) and $\tilde{\xi}$ (cf. Section 4.2.2). In particular, by (58) and the first line of (64), (66.2) holds. On the other hand, combining Lemma 4.2.2 and the second line of (64), we see that the assumption (67) holds, and therefore Lemma 4.2 .4 implies the validity of (66.1). The arguments in Section 1.2.7 then complete the last step of the proof of Proposition 2.1 (a).

4.3. Gluing broken orbits. We now discuss the modification needed for the proof of Proposition 2.1 (b).

Given a broken orbit $\left\{\hat{u}_{1}, \hat{u}_{2}, \ldots, \hat{u}_{k}\right\}$ connected at $y$, and an $\lambda \in\left(0, \lambda_{0}\right)$, the pregluing $w_{\chi}$ associated to $\chi=\left(\left\{\hat{u}_{1}, \hat{u}_{2}, \ldots, \hat{u}_{k}\right\}, \lambda\right) \in \Xi(\mathbb{S})$ is given by

$$
w_{\chi}=\underline{w}_{\chi}
$$

where $\underline{w}_{\chi}$ is given by the same formula (11), except that now $i \in\{1, \ldots, k\}$ only, and instead of taking values in $\mathbb{R}, s$ now takes value in $\mathbb{R} / T_{\chi} \mathbb{Z}$, where

$$
T_{\chi}:=\mathfrak{l}_{k+1} .
$$

With this explained, the material in Sections 2 and 3 transfers directly to the case of broken orbits, but the discussion in Sections 4.1 and 4.2 above requires the following modification.

4.3.1. Constructing the gluing map. At a closed orbit $(\lambda,(T, w)) \in$ $\mathcal{B}_{O}^{\Lambda}=\Lambda \times \mathcal{B}_{O}$, the deformation operator is $\hat{D}_{(\lambda,(T, w))}: \mathbb{R}_{\alpha} \oplus \mathbb{R}_{\varrho} \oplus L_{1}^{p}\left(w^{*} K\right) \rightarrow$ $L^{p}\left(w^{*} K\right)$,

$$
\hat{D}_{(\lambda,(T, w))}(\alpha, \varrho, \xi)=\alpha \partial_{\lambda} \check{\theta}_{X_{\lambda}}+\tilde{D}_{(T, w)}(\varrho, \xi),
$$

namely, it is a rank-2 stabilization of $D_{w}$ (cf. Section 3.3.1). $\left(\mathbb{R}_{\alpha}, \mathbb{R}_{\varrho}\right.$ above, respectively, parameterize variation in $\lambda$ and in the period $T$ ). In our context, this operator is a map between the weighted spaces

$$
\hat{W}_{\chi}:=\mathbb{R}_{\alpha} \oplus \mathbb{R}_{\varrho} \oplus W_{\chi} \text { and } L_{\chi} .
$$


Let $K_{\chi}=\operatorname{Span}\left\{\mathfrak{e}_{u_{i}}\right\}_{i=1}^{k}, C_{\chi}=\operatorname{Span}\left\{\mathfrak{f}_{j}\right\}_{j=1}^{k}$. The analog of Proposition 3.1.3 shows that $\left[K_{\chi} \rightarrow C_{\chi}\right]$ forms a K-model for $D_{w_{\chi}}$, which induces a K-model for $\hat{D}_{\left(\lambda,\left(T_{\chi}, w_{\chi}\right)\right)}$ by stabilization.

However, since $s$ is now periodic instead of real, the matrix $\lambda^{-1 / 2+1 /(2 p)}\left(P_{\mathfrak{f}_{j}} D_{w_{\chi}} \mathfrak{e}_{u_{i}}\right)$ is no longer (approximately) triangular, and hence not clearly uniformly invertible. Consequently, it is no longer clear that, with the choice of $Q_{\chi}$ in Section 4.1, reduction by $Q_{\chi}$ gives another K-model for the deformation operator. Instead, use the following subspace $Q_{O, \chi} \subset \hat{W}_{\chi}$ :

$$
Q_{O, \chi}=\mathbb{R}_{\alpha} \oplus * \oplus \operatorname{Span}\left\{\mathfrak{e}_{u_{i}}\right\}_{i=1}^{k-1} .
$$

Note that from (52) and the uniform boundedness of $P_{\mathfrak{f}_{j}}$

$$
C_{\alpha-} \lambda^{1 / 2-1 /(2 p)} \leq \lambda^{3 / 2} P_{\mathfrak{f}_{j}} \partial_{\lambda} \check{\theta}_{X_{\lambda}}\left(w_{\chi}\right) \leq C_{\alpha+} \lambda^{1 / 2-1 /(2 p)}
$$

for constants $C_{\alpha \pm}>0$ independent of $\lambda$. Supplementing Lemma 4.1.3 (b) with this additional estimate, we see that the matrix representation of the operator

$$
\left.\lambda^{-1 / 2+1 /(2 p)} \Pi_{C_{\chi}} \hat{D}_{\left(\lambda,\left(T_{\chi}, w_{\chi}\right)\right)}\right|_{Q_{O, \chi}}
$$

with respect to the bases

$$
\left\{\left(\lambda^{3 / 2}, 0,0\right),\left(0,0, \mathfrak{e}_{1}\right), \ldots,\left(0,0, \mathfrak{e}_{k-1}\right)\right\}, \quad\left\{\mathfrak{f}_{1}, \ldots, \mathfrak{f}_{k}\right\}
$$

is, modulo ignorable terms, of the form

$$
\left(\begin{array}{ccccc}
+ & - & 0 & \cdots & 0 \\
+ & + & - & 0 & 0 \\
+ & 0 & + & \ddots & \vdots \\
\vdots & \vdots & \ddots & \ddots & - \\
+ & 0 & \cdots & 0 & +
\end{array}\right) \quad(+/- \text { denote positive/negative numbers of } O(1))
$$

which is easily seen to have a uniformly bounded right inverse. Thus, the rest of Section 4.1 may be repeated with $Q_{\chi}$ replaced by $Q_{O, \chi}$ to define a gluing map in this case, which is also a local diffeomorphism.

4.3.2. Surjectivity of the gluing map. As the choice of $Q_{\chi^{\prime}}$ is changed, the definition of the $B$-space $B_{\chi^{\prime}}$ changes accordingly. In this situation,

$$
B_{\chi^{\prime}}=\left\{\left(\alpha^{\prime}, 0, \xi_{\chi^{\prime}}\right) \mid\left\langle\left(u_{k}\right)_{\gamma}(0),\left(\gamma_{u_{k}}^{-1}\right)^{*} \xi_{\chi^{\prime}}(0)\right\rangle_{2, t}=0\right\} \subset \hat{W}_{\chi^{\prime}} .
$$

(Note that in the case of broken orbits, $i \in \mathbb{Z} / k \mathbb{Z}$, thus $u_{0}=u_{k}$.) The work in Section 4.2 needs corresponding modification.

Given a $(\lambda,(T, w)) \in \hat{\mathcal{M}}_{O}^{\Lambda}$ close to the broken orbit $\left\{\hat{u}_{1}, \ldots, \hat{u}_{k}\right\}$, one may define $\tilde{w}$ and $\tilde{\gamma}_{u_{i}}$ in essentially the same way as Section 4.2 .1 , and the estimates in Section 4.2.2 still hold. However, for $\chi=\left(\left\{\hat{u}_{1}, \ldots, \hat{u}_{k}\right\}, \lambda\right)$, the period $T_{\chi}$ of the pregluing $w_{\chi}$ differs from those of $\tilde{w}$ or $w$. Thus, 
instead of comparing with $w_{\chi}$, we compare $w$ or $\tilde{w}$ with $w_{\chi^{\prime}}$, where $\chi^{\prime}=$ $\left(\left\{\hat{u}_{1}, \ldots, \hat{u}_{k}\right\}, \lambda^{\prime}\right)$, and $\lambda^{\prime}=\lambda+\alpha^{\prime}$ is chosen so that the period of $w_{\chi^{\prime}}$ agrees with the period of $w$ (which is also the period of $\tilde{w}$ ). With this choice of $\chi^{\prime}$, assumption (58), together with the definition of $B_{\chi^{\prime}}$ above, implies (66.2).

Moreover, the length estimates in Section 2.2.1 show that

$$
C_{-} \lambda^{-1 / 2} \leq T_{\chi} \leq C_{+} \lambda^{-1 / 2}
$$

combining with the estimate for the difference in periods of $w$ and $w_{\chi}$ given by (63), we have:

$$
\left|\alpha^{\prime}\right| \leq C \lambda^{3 / 2} \varepsilon
$$

For such $\lambda^{\prime}=\lambda+\alpha^{\prime}$, the difference $\gamma_{\lambda^{\prime}, u_{i}}^{-1}-\tilde{\gamma}_{u_{i}}^{-1}$ satisfies estimates similar to (63). Thus, (67) holds for this choice of $\chi^{\prime}$, which in turn implies (66.1), via Lemma 4.2.4.

\section{Gluing at births}

The purpose of this section is to prove Proposition 5.1 below. The proof is in many ways similar to the proof of Proposition 2.1, but simpler in Step 2, since here we glue only a single flow line, and the generalized cokernel in this case is trivial.

5.1. Statement of the gluing theorem. The next proposition verifies part of (RHFS2c, 3c) for admissible $(J, X)$-homotopies.

Proposition. Let $\left(J^{\Lambda}, X^{\Lambda}\right)$ be an admissible $(J, X)$-homotopy connecting two regular pairs, and $\mathbf{x}, \mathbf{z}$ be two path components of $\mathcal{P} \Lambda \backslash \mathcal{P} \Lambda, \operatorname{deg}$. Then a chain-topology neighborhood of $\mathbb{J}_{P}(\Lambda, \mathbf{x}, \mathbf{z} ; \Re)$ in $\hat{\mathcal{M}}_{P}^{\Lambda, 1,+}\left(\mathbf{x}, \mathbf{z} ; \mathrm{wt}_{-\langle y\rangle, e_{\mathcal{P}}} \leq \Re\right)$ is l.m.b. along $\mathbb{J}_{P}(\Lambda, \mathbf{x}, \mathbf{z} ; \Re)$.

Furthermore, $\Pi_{\Lambda}$ maps these neighborhoods to birth-neighborhoods.

We shall restrict our attention to

$$
\hat{u} \in \hat{\mathcal{M}}_{P, \lambda}^{0}((\underline{\mathbf{x},[\mathbf{w}]}),(\underline{\mathbf{z},[\mathbf{v}]})) \cap \mathbb{J}_{P}(\Lambda, \mathbf{x}, \mathbf{z} ; \Re),
$$

where one of $x_{\lambda}$ and $z_{\lambda}$ is a death-birth, and $\operatorname{gr}_{+}\left(\left(x_{\lambda},\left[w_{\lambda}\right]\right),\left(z_{\lambda},\left[v_{\lambda}\right]\right)\right)=$ $\operatorname{gr}((\mathbf{x},[\mathbf{w}]),(\mathbf{z},[\mathbf{v}]))$. The other cases follow either from standard gluing theory or from structure theory of parameterized moduli spaces, since the flow lines decay exponentially to the critical points in these cases.

Without loss of generality, assume as in Sections 2-4 that $\lambda=0$, that $z_{\lambda}=y$ is in a standard death-birth neighborhood, and that the $(J, X)$ homotopy is oriented such that $C_{y}^{\prime}>0$. Under these assumptions, a birth neighborhood is $\left(-\lambda_{0}, 0\right) \subset \Lambda$, for a small $\lambda_{0}>0$.

Our goal is thus to construct a gluing map from $\Xi(\mathbb{S})$ to $\hat{\mathcal{M}}_{P}^{\Lambda, 1}\left(\mathbf{x}, \mathbf{z} ; \mathrm{wt}_{-\langle y\rangle, e_{\mathcal{P}}} \leq \Re\right)$, where

$$
\mathbb{S}=\hat{\mathcal{M}}_{P, 0}^{0}\left(\underline{\mathbf{x}}, \underline{\mathbf{y}} ; \mathrm{wt}_{-}\langle y\rangle, e_{y} \leq \Re\right) ; \quad \Xi(\mathbb{S})=\mathbb{S} \times\left(-\lambda_{0}, 0\right) \quad \text { for a small } \lambda_{0}>0 .
$$


We shall again focus on a single $\hat{u} \in \mathbb{S}$, since in this case $\mathbb{S}$ also consists of finitely many isolated points. Notice that when $x_{0}=y, \hat{u}$ can be the constant flow at $y, \bar{y}$. The argument required for this case is somewhat different from the other cases. We discuss this case in Section 5.3, and the other cases in Section 5.2.

5.2. When $u \neq \bar{y}$. Assume without loss of generality that $x_{0} \neq y$ is nondegenerate, so that we may concentrate on the region where $s>0$.

5.2.1. Pregluing. Let $\chi:=(\lambda, \hat{u}) \in \Xi(\mathbb{S})$ as above, and let $u$ be a centered representative. Write

$$
u(s)=\exp (y, \mu(s)) \quad \text { for large } s,
$$

and as in I.5.3.2, let

$$
y_{\lambda-}=\exp \left(y, \eta_{\lambda-}\right) \in \mathcal{P}_{\lambda}
$$

be the critical point near $y$ of index ind $(y)$. Note that $\left\langle\mathbf{e}_{y}, \mu\right\rangle_{2, t}(s)>$ 0 is a decreasing function for large $s$, sending $\left(s_{0}, \infty\right)$ to $(C, 0)$ for some positive numbers $s_{0}, C$. Since $\left\langle\mathbf{e}_{y}, \eta_{\lambda_{-}}\right\rangle_{2, t}$ is a small positive number, it equals $\left\langle\mathbf{e}_{y}, \mu(s)\right\rangle_{2, t}$ for certain large $s=\breve{\gamma}_{\lambda}$. From the estimates in Lemma I.5.3.2 and Proposition I.5.1.3, we have

$$
C|\lambda|^{-1 / 2} \leq \breve{\gamma}_{\chi} \leq C^{\prime}|\lambda|^{-1 / 2}
$$

Let $R<\breve{\gamma}_{\chi}-1$ be a $\lambda$-independent large positive number such that $u(s)$ is close to $y$ for $s \geq R$, and set $R_{ \pm}= \pm C_{0}|\lambda|^{-1 / 2}$ for some $\lambda$-independent constant $C_{0}>0$. Define $u_{\lambda} \in \Gamma\left(\left(-\infty, \breve{\gamma}_{\chi}\right) \times S^{1}, p_{2}^{*} T_{f}\right)$ by

$$
u_{\lambda}(s):= \begin{cases}e_{R_{-}, R_{+}}(0, u ; \lambda, 0) & \text { when } s \leq R / 2, \\ \exp \left(y, \mu(s)+\beta(s-R) \Pi_{\mathrm{ker} A_{y}}^{\perp} \eta_{\lambda-}\right) & \text { when } s \geq R / 2,\end{cases}
$$

Lemma. There is a function $\gamma_{\chi}(s)$ defining a homeomorphism from $\mathbb{R}$ to $\left(-\infty, \breve{\gamma}_{\chi}\right)$, such that

$$
\begin{cases}\left\langle w_{\chi}^{\prime}(s), \bar{\partial}_{J, X_{\lambda}} w_{\chi}(s)\right\rangle_{2, t}=0 ; & \text { when } s \in\left[\gamma_{\chi}^{-1}(0), \infty\right) \\ \gamma_{\chi}^{\prime}=1 & \text { otherwise }\end{cases}
$$

Proof. Write $\frac{d \gamma_{\chi}}{d s}=h\left(\gamma_{\chi}\right)$, where

$$
h(\gamma):= \begin{cases}1-\left\langle\left(u_{\lambda}\right)_{\gamma}, \bar{\partial}_{J X_{\lambda}}\left(u_{\lambda}\right)\right\rangle_{2, t}\left\|\left(u_{\lambda}\right)_{\gamma}\right\|_{2, t}^{-2} & \text { when } s \in\left[\gamma_{\chi}^{-1}(0), \infty\right) ; \\ 1 & \text { otherwise. }\end{cases}
$$

We now examine the behavior of $\bar{\partial}_{J X_{\lambda}} u_{\lambda}(\gamma)$ near $\gamma=\breve{\gamma}_{\chi}$. Here since $u_{\lambda}$ is close to $y_{\lambda_{-}}$, expanding $\bar{\partial}_{J X_{\lambda}}$ about $y_{\lambda_{-}}$and writing $\exp \left(y_{\lambda_{-}}, \mu_{\lambda}(\gamma)\right)=$ $u_{\lambda}(\gamma)$, we have

$$
T_{u_{\lambda}, y_{\lambda-}} \bar{\partial}_{J X_{\lambda}} u_{\lambda}(\gamma)=\left(\mu_{\lambda}\right)_{\gamma}+A_{y_{\lambda-}} \mu_{\lambda}+n_{y_{\lambda-}}\left(\mu_{\lambda}\right)
$$


By definition, $\mu_{\lambda}\left(\breve{\gamma}_{\chi}\right)=0$; hence $h\left(\breve{\gamma}_{\chi}\right)=0$. Thus,

$$
\begin{aligned}
h(\gamma)=\langle & \left(u_{\lambda}\right)_{\gamma}(\gamma), T_{y_{\lambda-}, u_{\lambda}} A_{y_{\lambda-}} T_{y_{\lambda}, u_{\lambda}}^{-1}\left(\left(\breve{\gamma}_{\chi}-\gamma\right)\left(\left(u_{\lambda}\right)_{\gamma}(\gamma)\right)\right\rangle_{2, t}\left\|\left(u_{\lambda}\right)_{\gamma}(\gamma)\right\|_{2, t}^{-2} \\
& +O\left(\left|\breve{\gamma}_{\chi}-\gamma\right|^{2}\left\|\left(u_{\lambda}\right)_{\gamma}(\gamma)\right\|_{2,1, t}\right) .
\end{aligned}
$$

By the estimate for minimal eigenvalue of $A_{y_{\lambda_{-}}}$in I.5.3.2, this is bounded above and below by multiples of $|\lambda|^{1 / 2}\left(\breve{\gamma}_{\chi}-\gamma\right)$. Integrating like (15), we see that for large $s$

$$
C_{5}^{\prime} \mathrm{e}^{-c_{6}^{\prime}|\lambda|^{1 / 2} s} \leq \breve{\gamma}_{\chi}-\gamma_{\chi} \leq C_{5} \mathrm{e}^{-c_{6}|\lambda|^{1 / 2} s},
$$

while on the other end $\gamma_{\chi}(s)=s+c_{\lambda}$ for some constant $c_{\lambda}$. We define $\gamma_{\chi}$ such that $\gamma_{\chi}(s)=s$ for $s<0$.

Definition. The pregluing $w_{\chi}$ corresponding to gluing data $\chi=(\lambda, u)$ is

$$
w_{\chi}(s):=u_{\lambda}\left(\gamma_{\chi}(s)\right) \text {. }
$$

5.2.2. The weighted norms. The norms $W_{\chi}, L_{\chi}$ here are defined by the same formulae in Definition 2.3.1, with the weight function $\sigma_{\chi}$ replaced by

$$
\sigma_{\chi}(s):= \begin{cases}\left\|w_{\chi}^{\prime}\left(\gamma_{\chi}^{-1}(0)\right)\right\|_{2, t}^{-1} & \text { when } s \leq \gamma_{\chi}^{-1}(0), \\ \left\|w_{\chi}^{\prime}(s)\right\|_{2, t}^{-1} & \text { when } \gamma_{\chi}^{-1}(0) \leq s \leq \gamma_{\chi}^{-1}\left(\mathfrak{r}_{\chi}\right), \\ \left\|w_{\chi}^{\prime}\left(\gamma_{\chi}^{-1}\left(\mathfrak{r}_{\chi}\right)\right)\right\|_{2, t}^{-1} & \text { when } s \geq \gamma_{\chi}^{-1}\left(\mathfrak{r}_{\chi}\right),\end{cases}
$$

where $\mathfrak{r}_{\chi}=\mathfrak{r}_{\chi}(\lambda, \epsilon)=C_{\tau}(\lambda / \epsilon)^{-1 / 2}<\breve{\gamma}_{\chi}$ is chosen such that $1-h(s) \leq \epsilon$ where $s \leq \mathfrak{r}_{\chi}$ for a small positive number $\epsilon$.

We shall frequently call on the following useful facts.

(a) In this case, $\gamma_{\chi}^{\prime} \leq 1$.

(b) $\left\|\Pi_{\text {ker } A_{y}}^{\perp} \eta_{\lambda-}\right\|_{2,2, t} \leq C|\lambda|$ by I.(55), Lemma I.5.3.2, and the decay estimates in Proposition I.5.1.3.

(c) $\sigma_{\chi} \leq C|\lambda|^{-1}$.

In particular, Fact (b) often implies that in addition to estimates analogous to those in the proof of Proposition 2.1, the extra terms introduced by the cutoff function $\beta$ in the definition of $u_{\lambda}$ is usually ignorable.

5.2.3. Error estimate. Proceeding to Step 1 of the gluing theory, we have Lemma 5.2.3.

Lemma. $\left\|\bar{\partial}_{J X_{\lambda}} w_{\chi}\right\|_{L_{\chi}} \leq C \lambda^{1 / 2-1 /(2 p)}$.

Proof. Consider the two regions (a) $\gamma_{\chi}^{-1}\left(-R_{-}\right) \leq s \leq \gamma_{\chi}^{-1}(R)$, (b) $\gamma_{\chi}^{-1}(R) \leq$ $s \leq \infty$ separately. The point is to expand $\bar{\partial}_{J X_{\lambda}} w_{\chi}(s)=\tilde{\Pi}_{u_{\lambda}^{\prime}}^{\perp}\left(\bar{\partial}_{J H_{\lambda}} u_{\lambda}\left(\gamma_{\chi}(s)\right)\right)$ differently in the two regions: expand $u_{\lambda}$ about $u$ in region (a) and about $y_{\lambda}$ in region (b). 
In region (a), modulo terms coming from $\beta\left(\gamma_{\chi}-R\right) \Pi_{\text {ker } A_{y}}^{\perp} \eta_{\lambda-}$, the estimate of the norm is entirely parallel to that in Proposition 2.4.1. The time $w_{\chi}$ spends in this region is

$$
\gamma_{\chi}^{-1}(R)-\gamma_{\chi}^{-1}\left(R_{-}\right) \leq C|\lambda|^{-1 / 2}
$$

the $L^{\infty}$-norm of $\sigma_{\chi} \bar{\partial}_{J X_{\lambda}} w_{\chi}$ can be estimated as in Case 1 of Lemma 2.4.2, with $\gamma_{0}$ replaced by $R$.

On the other hand, since $\sigma_{\chi}$ has a $\lambda$-independent uniform bound in this region, and the $L_{t}^{p}$-norm of the contribution to $\bar{\partial}_{J X_{\lambda}} w_{\chi}$ from the extra terms introduced by $\beta \Pi_{\mathrm{ker} A_{y}}^{\perp} \eta_{\lambda-}$ can be bounded by $C\left\|\Pi_{\mathrm{ker} A_{y}}^{\perp} \eta_{\lambda-}\right\|_{2,1, t} \leq C^{\prime}|\lambda|$, the contribution from these terms to $\left\|\sigma_{\chi} \bar{\partial}_{J X_{\lambda}} w_{\chi}\right\|_{p}$ is thus bounded by $C|\lambda|$.

For region (b), $w_{\chi}$ spends infinite amount of time here; however

$$
\begin{array}{r}
\Pi_{\left(u_{\lambda}\right)_{\gamma}}^{\perp} \bar{\partial}_{J X_{\lambda}} u_{\lambda}(\gamma)=\Pi_{\left(u_{\lambda}\right)_{\gamma}}^{\perp}\left((\delta \gamma) T_{y_{\lambda_{-}}, u_{\lambda}} A_{y_{\lambda}}\left(T_{y_{\lambda_{-}}, u_{\lambda}}\right)^{-1}\left(u_{\lambda}\right)_{\gamma}(\bar{\gamma})\right) \\
\left.+T_{y_{\lambda_{-}}, u_{\lambda}} n_{y_{\lambda}}\left((\delta \gamma)\left(T_{y_{\lambda_{-}}, u_{\lambda}}\right)^{-1}\left(u_{\lambda}\right)_{\gamma}(\bar{\gamma})\right)\right),
\end{array}
$$

where $\delta \gamma:=\breve{\gamma}_{\chi}-\gamma ; \gamma \leq \bar{\gamma} \leq \breve{\gamma}_{\chi}$. On the other hand, in this region, $\sigma_{\chi}(s) \leq$ $C|\lambda|^{-1}$. Thus by Lemma I.5.3.2, on this region $\left\|\sigma_{\chi} \bar{\partial}_{J X_{\lambda}} w_{\chi}\right\|_{p}$ is bounded by $C\|(\delta \gamma)\|_{p}|\lambda|^{1 / 2} \leq C^{\prime}|\lambda|^{1 / 2-1 /(2 p)}$, since $\delta \gamma \leq C_{5} \mathrm{e}^{-C_{6}|\lambda|^{1 / 2} s}$ by $(73)$.

5.2.4. Existence and uniform boundedness of the right inverse $G_{\chi}: L_{\chi} \rightarrow W_{\chi}$ of $E_{w_{\chi}}$. We now proceed to Step 2 of the proof. In this case, $W_{\chi}^{\prime} \subset W_{\chi}$ is

$$
W_{\chi}^{\prime}:=\left\{\xi \in W_{\chi} \mid\left\langle\nu(0), \xi\left(\gamma_{\chi}^{-1}(0)\right)\right\rangle_{2, t}=0 \text { for all } \nu \in \text { ker } E_{u}\right\},
$$

and we aim to show that there is a uniformly bounded isomorphism $G_{\chi}: L_{\chi} \rightarrow W_{\chi}^{\prime}$ which is a right inverse of $E_{w_{\chi}}$. Assume the opposite that there is a sequence $\left\{\xi_{\lambda} \in W_{\chi}^{\prime}\right\}_{\lambda}$ satisfying

$$
\begin{gathered}
\left\|\xi_{\lambda}\right\|_{W_{\chi}}=1 \\
\left\|E_{w_{\chi}} \xi_{\lambda}\right\|_{L_{\chi}}=: \varepsilon_{E}(\lambda) \rightarrow 0 \quad \text { when } \lambda \rightarrow 0
\end{gathered}
$$

Divide $\Theta=\mathbb{R} \times S^{1}$ into two parts $\Theta_{u}, \Theta_{y-}$, separated by the line $s=$ $\gamma_{\chi}^{-1}\left(\mathfrak{r}_{\chi}\right)$. Let $\Theta_{u}^{\prime}:=\left(-\infty, \gamma_{\chi}^{-1}\left(\mathfrak{r}_{\chi}\right)+1\right) \times S^{1} \supset \Theta_{u} ; \Theta_{y-}^{\prime}:=\left(\gamma_{\chi}^{-1}\left(\mathfrak{r}_{\chi}-\right.\right.$ $1), \infty) \times S^{1} \supset \Theta_{y-}$.

On $\underline{\Theta_{u}^{\prime}}$, we define $\xi_{\lambda, u} \in \Gamma\left(u^{*} K\right)$ by

$$
T_{u, u_{\lambda}} \xi_{\lambda, u}\left(\gamma_{\chi}(s)\right)=\xi_{\lambda}(s)
$$

Let $\left(\xi_{\lambda, u}\right)_{L}$ be the projection of $\xi_{\lambda, u}$ to the direction of $u^{\prime}$ and let $\left(\xi_{\lambda, u}\right)_{T}=$ $\xi_{\lambda, u}-\left(\xi_{\lambda, u}\right)_{L}$. Let $\beta_{u}$ be a smooth cutoff function supported on $\gamma_{\chi}\left(\Theta_{u}^{\prime}\right)$ with 
value 1 on $\gamma_{\chi}\left(\Theta_{u}\right)$. Arguing as in the proof of Proposition 3.1.3, one obtains:

$$
\begin{aligned}
\left\|\xi_{\lambda}\right\|_{W_{\chi}\left(\Theta_{u}\right)} \leq C\left\|\xi_{\lambda, u}\right\|_{W_{u}\left(\gamma_{\chi}\left(\Theta_{u}\right)\right)} & \leq C^{\prime}\left\|\beta_{u} E_{u} \xi_{\lambda, u}\right\|_{L_{u}\left(\gamma_{\chi}\left(\Theta_{u}^{\prime}\right)\right)}+C\left\|\beta_{u}^{\prime}\left(\xi_{\lambda, u}\right)_{T}\right\|_{L_{u}\left(\gamma_{\chi}\left(\Theta_{u}^{\prime}\right)\right)} \\
& \leq C^{\prime}(1+2 \epsilon)\left\|E_{w_{\chi}}\left(\xi_{\lambda}\right)\right\|_{L_{\chi}\left(\Theta^{\prime}\right)}+C^{\prime \prime}\left(\epsilon+|\lambda|^{1 / 2}\right)\left\|\xi_{\lambda}\right\|_{W_{\chi}} \\
& \quad+C\left\|\beta_{u}^{\prime}\left(\xi_{\lambda, u}\right)_{T}\right\|_{L_{u}\left(\gamma_{\chi}\left(\Theta_{u}^{\prime}\right)\right)} \\
& \leq 2 C^{\prime} \varepsilon_{E}+C^{\prime \prime}\left(\epsilon+|\lambda|^{1 / 2}\right)+C \varepsilon_{0} .
\end{aligned}
$$

(Note in comparison with (25), the second term in the third line above has a worse factor of $|\lambda|^{1 / 2}$ instead of $|\lambda|$; this arises from the difference between $u$ and $u_{\lambda}$.)

On the other hand, on $\Theta_{y-}^{\prime}$ we consider $\xi_{\lambda, y-} \in \Gamma\left(\bar{y}_{\lambda-}^{*} K\right)$ defined by

$$
T_{\bar{y}_{\lambda-}, w_{\chi}} \xi_{\lambda, y-}=\xi_{\lambda} .
$$

Let $\mathbf{e}_{y_{\lambda-}}$ be the unit eigenvector associated with the minimal eigenvalue of $A_{y_{\lambda-}}$, which goes to $\mathbf{e}_{y}$ as $\lambda \rightarrow 0$. By I.(57), $\mathbf{e}_{y_{\lambda_{-}}}$differs from

$T_{w_{\chi}, y_{\lambda-}} w_{\chi}^{\prime} /\left\|w_{\chi}^{\prime}\right\|_{2, t}(s)$ by $O\left(|\lambda|^{1 / 2}\right)$ for $s \in \Theta_{y-}^{\prime}$. Let

$$
\left(\xi_{\lambda, y-}\right)_{L}:=\Pi_{\mathbf{e}_{y_{\lambda-}}} \xi_{\lambda, y-}=\underline{\left(\xi_{\lambda, y-}\right)}{ }_{L} \mathbf{e}_{y_{\lambda-}},
$$

and $\left(\xi_{\lambda, y-}\right)_{T}=\xi_{\lambda, y-}-\left(\xi_{\lambda, y-}\right)_{L}$. The above observation about $\mathbf{e}_{y_{\lambda-}}$, together with the fact that in this region $\sigma_{\chi}$ is bounded above and below by multiples of $|\lambda|^{-1}$, implies that to estimate $\|\xi\|_{W_{\chi}\left(\Theta_{y_{-}}\right)}$or $\|\xi\|_{L_{\chi}\left(\Theta_{y_{-}}\right)}$, it is equivalent to estimate $\left\|\xi_{y-}\right\|_{W_{y-}\left(\Theta_{y-}\right)}$ or $\left\|\xi_{y-}\right\|_{L_{y-}\left(\Theta_{y-}\right)}$, where

$$
\begin{aligned}
\left\|\xi_{y-}\right\|_{W_{y-}} & :=|\lambda|^{-1 / 2}\left\|\xi_{y-}\right\|_{p, 1}+|\lambda|^{-1}\left\|\left(\xi_{y-}\right)_{L}^{\prime}\right\|_{p}, \\
\left\|\xi_{y-}\right\|_{L_{y-}} & :=|\lambda|^{-1 / 2}\left\|\xi_{y-}\right\|_{p, 1}+|\lambda|^{-1}\left\|\left(\xi_{y-}\right)_{L}\right\|_{p} .
\end{aligned}
$$

We have a refined version of Lemma 3.3.1 in this case.

Lemma (Refining Floer's lemma). Let $\xi_{\lambda}$ be as in (74). Then for all sufficiently small $\lambda$,

$$
|\lambda|^{1 /(2 p)}\left\|\xi_{\lambda, y-}\right\|_{L^{\infty}\left(\Theta_{y-}^{\prime}\right)}+\left\|\left(\xi_{\lambda, y-}\right)_{L}\right\|_{L^{\infty}\left(\Theta_{y-}^{\prime}\right)} \leq \varepsilon_{0}(\lambda)|\lambda|^{1 / 2+1 /(2 p)},
$$

where $\varepsilon_{0}(\lambda)$ is a small positive number, with $\lim _{\lambda \rightarrow 0} \varepsilon_{0}(\lambda)=0$.

Proof. The estimate for $\left\|\xi_{\lambda, y-}\right\|_{L^{\infty}\left(\Theta_{y-}^{\prime}\right)}$ follows easily from the argument for Lemma 3.3.1. The longitudinal component has a more refined bound because it has a better bound on the Sobolev norm. Let

$$
\tilde{\varsigma}_{\lambda}(\tau):=\lambda^{-1 / 2-1 /(2 p)} \underline{\left(\xi_{\lambda, y-}\right)} L^{\left(\lambda^{-1 / 2}\left(\tau+s_{\lambda}\right)\right)} \text { over }[1, \infty),
$$

where $s_{\lambda}$ are constants chosen such that $\lambda^{-1 / 2}\left(1+s_{\lambda}\right)=\gamma_{\chi}^{-1}\left(\mathfrak{r}_{\chi}-1\right)$. Then by $(74),\left\|\tilde{\varsigma}_{\lambda}\right\|_{L_{1}^{p}([1, \infty))}$ is bounded (note the rescaling contributes a factor of 
$(\lambda)^{-1 /(2 p)}$ to the $L_{1}^{p}$-norm). Thus (again after possibly taking a subsequence) $\tilde{\varsigma}_{\lambda}$ converges in $C_{0}$ to $\tilde{\varsigma}_{0}$, and $s_{\lambda} \rightarrow s_{0}$. $\tilde{\varsigma}_{0}$ satisfies an equation of the form

$$
\frac{d \tilde{\varsigma}_{0}}{d \tau}+\chi \tilde{\varsigma}_{0}=0, \quad \text { where } \chi \sim C_{1}+C_{2} \mathrm{e}^{-\nu^{\prime} \tau} .
$$

(The assumption of being in a standard d-b neighborhood is used to simplify the differential equation above. Notice also that $\left(\xi_{\lambda}\right)_{T}$ does not appear in this equation, because by the $L_{t}^{\infty}$ estimate for $\xi_{\lambda}$, its contribution vanishes as $\lambda \rightarrow 0$.) Thus, $\left\|\tilde{\varsigma}_{0}\right\|_{\infty} \leq\left|\tilde{\varsigma}_{0}(1)\right|$. Meanwhile, $\tilde{\varsigma}_{0}(1)=0$ since by the argument for $(35),\left\|\left(\xi_{\lambda}\right)_{L}\left(\gamma_{\chi}^{-1}\left(\mathfrak{r}_{\chi}-1\right)\right)\right\|_{\infty, t} \leq C \lambda^{1 / 2+1 /(2 p)} \varepsilon_{u}$.

Let $\beta_{y-}$ be a cutoff function on $\mathbb{R}$ which vanishes in $\left(-\infty, \mathfrak{r}_{\chi}-1\right]$ and is 1 on $\left[\mathfrak{r}_{\chi}, \infty\right)$. We may estimate the longitudinal component as:

$$
\begin{aligned}
& \quad|\lambda|^{-1}\left\|\left(\xi_{\lambda, y-}\right)_{L}^{\prime}\right\|_{L^{p}\left(\Theta_{y-}\right)}+|\lambda|^{-1 / 2}\left\|\left(\xi_{\lambda, y-}\right)_{L}\right\|_{L^{p}\left(\Theta_{y-}\right)} \\
& \leq C|\lambda|^{-1}\left\|E_{y_{\lambda}}\left(\beta_{y-}\left(\gamma_{\chi}\right)\left(\xi_{\lambda, y-}\right){ }_{L}\right)\right\|_{L^{p}\left(\Theta_{y-}^{\prime}\right)} \\
& \leq C|\lambda|^{-1}\left\|\beta_{y-}\left(\gamma_{\chi}\right)\left(E_{y_{\lambda-}}\left(\xi_{\lambda, y-}\right)_{L}\right)_{L}\right\|_{L^{p}\left(\Theta_{y-}^{\prime}\right)} \\
& \quad+C^{\prime}|\lambda|^{-1}\left\|\left(\beta_{y-}\right)_{\gamma}\left(\gamma_{\chi}\right) \gamma_{\chi}^{\prime}\left(\xi_{\lambda, y-}\right)_{L}\right\|_{L^{p}\left(\Theta_{y-}^{\prime}\right)} \\
& \leq C_{1}\left\|\beta_{y-} \circ \gamma_{\chi} E_{w_{\chi}} \xi_{\lambda}\right\|_{L_{\chi}\left(\Theta_{y-}^{\prime}\right)} \\
& \quad+C_{2}|\lambda|^{-1 / 2}\left\|\beta_{y-}\left(\gamma_{\chi}\right) \mathrm{e}^{-C_{6}|\lambda|^{-1 / 2}\left(s-\gamma_{\chi}^{-1}\left(\mathfrak{r}_{\chi}\right)\right)}\left(\xi_{\lambda, y-}\right)_{L}\right\|_{L^{p}\left(\Theta_{y-}^{\prime}\right)} \\
& \quad+C^{\prime}|\lambda|^{-1}\left\|\left(\beta_{y-}\right)_{\gamma}\left(\gamma_{\chi}\right) \gamma_{\chi}^{\prime}\left(\xi_{\lambda, y-}\right)_{L}\right\|_{L^{p}\left(\Theta_{y-}^{\prime}\right)} \\
& \quad+C_{3}\left\|\beta_{y-}\left(\gamma_{\chi}\right) \mathrm{e}^{-C_{6}|\lambda|^{-1 / 2}\left(s-\gamma_{\chi}^{-1}\left(\mathfrak{r}_{\chi}\right)\right)}\left(\xi_{\lambda, y-}\right)_{T}\right\|_{L^{p}\left(\Theta_{y-}^{\prime}\right)} \\
& \leq C_{1}^{\prime} \varepsilon_{E}+C_{2}^{\prime} \varepsilon_{0} .
\end{aligned}
$$

The first inequality above follows from the eigenvalue estimate for $A_{y_{\lambda_{-}}}$in I.5.3.2. The second term in the penultimate expression above comes from the difference between $E_{w_{\chi}}$ and (a conjugate of) $E_{y_{\lambda_{-}}}$, while the last term arises from $\left(T_{\bar{y}_{\lambda-}, w_{\chi}}^{-1} E_{w_{\chi}} T_{\bar{y}_{\lambda-}, w_{\chi}}\left(\xi_{\lambda, y_{-}-}\right)_{T}\right)_{L}$. (Note that this term would have an extra factor of $|\lambda|^{-1 / 2}$ if I.5.3.1 (1c) is not assumed.) We have also used Lemma 5.2.4 and the estimates that in this region,

$$
\begin{array}{r}
\left|\left(\beta_{y-}\right)_{\gamma} \gamma_{\chi}^{\prime}\right| \leq C|\lambda|^{1 / 2} \exp \left(-C_{6}|\lambda|^{1 / 2}\left(s-\gamma_{\chi}^{-1}\left(\mathfrak{r}_{\chi}\right)\right)\right) \quad \text { and that } \\
\left\|\mu_{\lambda}\left(\gamma_{\chi}(s)\right)\right\|_{2,2, t} \leq C^{\prime}|\lambda|^{1 / 2} \exp \left(-C_{6}|\lambda|^{1 / 2}\left(s-\gamma_{\chi}^{-1}\left(\mathfrak{r}_{\chi}\right)\right),\right.
\end{array}
$$

which in turn follows from the computation in the proof of Lemma 5.2.1.

Similarly, the transversal direction can be estimated by:

$$
\begin{aligned}
& |\lambda|^{-1 / 2}\left(\left\|\left(\xi_{\lambda, y-}\right)_{T}^{\prime}\right\|_{L^{p}\left(\Theta_{y-}\right)}+\left\|\left(\xi_{\lambda, y-}\right)_{T}\right\|_{L^{p}\left(\Theta_{y-}\right)}\right) \\
& \quad \leq C\left\|\beta_{y_{-}}\left(\gamma_{\chi}\right) E_{w_{\chi}} \xi_{\lambda}\right\|_{L_{\chi}\left(\Theta_{y-}^{\prime}\right)}+C^{\prime}|\lambda|^{1 / 2-1 /(2 p)} \varepsilon_{0}(\lambda) \\
& \quad \leq C^{\prime \prime} \varepsilon_{E}+C^{\prime}|\lambda|^{1 / 2-1 /(2 p)} \varepsilon_{0} .
\end{aligned}
$$


Combining (77), (78), and (75), we obtain $\left\|\xi_{\lambda}\right\|_{W_{\chi}} \ll 1$ for all large enough $\lambda$, and hence the desired contradiction.

5.2.5. Surjectivity of the gluing map. Estimates for the nonlinear terms required for Step 3 in this case are not very different from those discussed in Section 2.5, and hence will be omitted. The argument in Section 1.2.1 then defines a gluing map, which is a local diffeomorphism onto a $\mathcal{B}$-topology neighborhood of the image of pregluing map. Again, we need to show that the latter neighborhood contains a chain-topology neighborhood of $\mathbb{S}$.

To adapt the proof in Section 4.2, given $(\lambda, \hat{w}) \in \hat{\mathcal{M}}^{1, \Lambda}\left(\mathbf{x}, \mathbf{y}_{\lambda-}\right)$ close to $\hat{u} \in$ $\mathbb{S}$ in the chain-topology neighborhood, we may again choose a representative $w$ and $\tilde{w}$ as in Section 4.2.1, satisfying conditions similar to (57) and (58).

- $\tilde{w}(s):=u_{\lambda}\left(\tilde{\gamma}_{\chi}(s)\right)$, where $\tilde{\gamma}_{\chi}: \mathbb{R} \rightarrow\left(-\infty, \breve{\gamma}_{\chi}\right)$ is a homeomorphism determined by

$$
\Pi_{\mathbf{e}_{y}} \tilde{\zeta}_{\lambda}(s)=\Pi_{\mathbf{e}_{y}} \zeta(s) \quad \forall s \in\left[\tilde{\gamma}_{\chi}^{-1}(R+1), \infty\right),
$$

and $\zeta, \tilde{\zeta}_{\lambda}$ are defined by $w(s)=\exp (y, \zeta(s)), \tilde{w}(s)=\exp \left(y, \tilde{\zeta}_{\lambda}(s)\right)$ as in Section 4.2.1.

- $\gamma_{\chi}^{-1}(0)=\tilde{\gamma}_{\chi}^{-1}(0) ; \quad\left\langle u_{\gamma}(0), \tilde{\gamma}_{\chi}^{*} \tilde{\xi}(0)\right\rangle_{2, t}=0$.

Equation (59) is in this case replaced by the following lemma.

Lemma. $\forall s \in\left[\tilde{\gamma}_{\chi}^{-1}(R+1), \infty\right)$,

$$
\begin{aligned}
& \|\tilde{\xi}(s)\|_{2,2, t}+\left\|\tilde{\xi}^{\prime}(s)\right\|_{2,1, t} \\
& \quad \leq C\left(|\lambda|+\left\|\Pi_{\mathbf{e}_{y}}\left(\tilde{\zeta}_{\lambda}(s)-\tilde{\zeta}_{\lambda}(\infty)\right)\right\|_{2, t}^{3}\right)\left\|\Pi_{\mathbf{e}_{y}}\left(\tilde{\zeta}_{\lambda}(s)-\tilde{\zeta}_{\lambda}(\infty)\right)\right\|_{2, t} .
\end{aligned}
$$

Proof. Write $u\left(\tilde{\gamma}_{\chi}(s)\right)=\exp (y, \tilde{\zeta}(s))$, and let $b(s):=\Pi_{\mathbf{e}_{y}} \tilde{\zeta}(s), c(s):=\zeta(s)-$ $\tilde{\zeta}(s)$. Note that $\Pi_{\mathbf{e}_{y}} \tilde{\zeta}=\Pi_{\mathbf{e}_{y}} \tilde{\zeta}_{\lambda}$, and on this region $\tilde{\zeta}_{\lambda}-\tilde{\zeta}=\eta_{\lambda-} \forall s$. The functions $b(s), c(s)$ still satisfy $(62),(61)$. However, we want to estimate instead

$$
c_{d}(s):=\zeta(s)-\tilde{\zeta}_{\lambda}(s)=c(s)-\Pi_{\mathrm{ker} A_{y}}^{\perp} \eta_{\lambda-} .
$$

From the definitions, estimates for $c_{d}$ would imply similar estimates for $\tilde{\xi}$.

Let $\underline{b}_{d}(s):=\left\langle\mathbf{e}_{y}, \tilde{\zeta}_{\lambda}(s)-\tilde{\zeta}_{\lambda}(\infty)\right\rangle_{2, t} ; b_{d}(s)=\underline{b}_{d}(s) \mathbf{e}_{y}$. Noting that

$$
\begin{aligned}
& -A_{y} \Pi_{\mathrm{ker} A_{y}}^{\perp} \eta_{\lambda-} \\
& =\left(1-\Pi_{\text {ker } A_{y}}-\nabla_{b(\infty)} Z \Pi_{\operatorname{ker} A_{y}}\right)\left(\hat{n}_{(0, y)}(\lambda, \tilde{\zeta}(\infty)+c(\infty))-n_{y}(\tilde{\zeta}(\infty))\right) \\
& \quad-\nabla_{b(\infty)} Z\left(\lambda C_{y}^{\prime} \mathbf{e}_{y}\right),
\end{aligned}
$$

we see that (61) may be rewritten in terms of $c_{d}$ as:

$$
\begin{aligned}
-c_{d}^{\prime}= & A_{y} c_{d}-\nabla_{b_{d}} Z\left(\lambda C_{y}^{\prime} \mathbf{e}_{y}+\Pi_{\operatorname{ker} A_{y}}\left(\hat{n}_{(0, y)}\left(\lambda, \tilde{\zeta}_{\lambda}(\infty)\right)-n_{y}(\tilde{\zeta}(\infty))\right)\right) \\
& +\left(1-\Pi_{\text {ker } A_{y}}-\nabla_{b} Z \Pi_{\text {ker } A_{y}}\right)\left(\begin{array}{l}
\hat{n}_{(0, y)}\left(\lambda, \tilde{\zeta}_{\lambda}+c_{d}\right)-n_{y}(\tilde{\zeta}) \\
-\hat{n}_{(0, y)}\left(\lambda, \tilde{\zeta}_{\lambda}(\infty)\right)+n_{y}(\tilde{\zeta}(\infty))
\end{array}\right)
\end{aligned}
$$


By the nature of $u, u_{\lambda}$, and $\hat{n}_{(0, y)}$, this leads to the familiar estimates:

$$
\begin{gathered}
\left\|c_{d+}\right\|_{2, t}^{\prime} \geq \nu_{+}\left\|c_{d+}\right\|_{2, t}-\epsilon_{+}\left\|c_{d}\right\|_{2, t}-C_{+}\left|\lambda \underline{b}_{d}\right| ; \\
\left\|c_{d-}\right\|_{2, t}^{\prime} \leq-\nu_{-}\left\|c_{d-}\right\|_{2, t}+\epsilon_{-}\left\|c_{d}\right\|_{2, t}+C_{-}\left|\lambda \underline{b}_{d}\right| .
\end{gathered}
$$

Subtracting the two inequalities, we get

$$
\left(\left\|c_{d+}\right\|_{2, t}-\left\|c_{d-}\right\|_{2, t}\right)^{\prime} \geq \nu^{\prime}\left(\left\|c_{d+}\right\|_{2, t}-\left\|c_{d-}\right\|_{2, t}\right)-C^{\prime}\left|\lambda \underline{b}_{d}\right| .
$$

Taking convolution product with the integral kernel of $d / d s-\nu^{\prime}$, we find that for $s \geq s_{0}$

$$
\begin{aligned}
& \left\|c_{d+}\right\|_{2, t}(s)-\left\|c_{d-}\right\|_{2, t}(s) \\
& \quad \geq\left(\left\|c_{d+}\right\|_{2, t}\left(s_{0}\right)-\left\|c_{d-}\right\|_{2, t}\left(s_{0}\right)\right) \mathrm{e}^{\nu^{\prime}\left(s-s_{0}\right)}-C^{\prime} \int_{s_{0}}^{s}\left|\lambda \underline{b}_{d}(\underline{s})\right| \mathrm{e}^{\nu^{\prime}(s-\underline{s})} d \underline{s},
\end{aligned}
$$

and since $\underline{b}_{d}(s)>0$ decreases with $s$, this implies that for all large enough $s$,

$$
\left\|c_{d+}\right\|_{2, t}(s) \leq\left\|c_{d-}\right\|_{2, t}(s)+C^{\prime \prime}\left|\lambda \underline{b}_{d}(s)\right|
$$

otherwise $\left\|c_{d+}\right\|_{2, t}(s)-\left\|c_{d-}\right\|_{2, t}(s)$ would be growing exponentially as $s \rightarrow \infty$, contradicting the fact that by construction, $\lim _{s \rightarrow \infty}\left\|c_{d}(s)\right\|_{2, t}=0$.

Plugging in this back to (82), we get

$$
\left\|c_{d-}\right\|_{2, t}^{\prime} \leq-\nu_{-}^{\prime}\left\|c_{d-}\right\|_{2, t}+C_{-}^{\prime}\left|\lambda \underline{b}_{d}\right|
$$

where $\nu_{-}^{\prime}$ is a positive numbers close to $\nu_{-}$. Taking convolution product with the integral kernel of $d / d s+\nu^{\prime}$,

$$
\left\|c_{d-}(s)\right\|_{2, t}(s) \leq C_{0} \mathrm{e}^{-\nu_{-}^{\prime} s}+\int_{s_{0}}^{s}|\lambda| \underline{b}_{d}(\underline{s}) \mathrm{e}^{\nu_{-}^{\prime}(\underline{s}-s)} d \underline{s} .
$$

We claim that there is a positive constant $\nu_{-}^{\prime \prime}$ slightly smaller than $\nu_{-}^{\prime}$ such that

$$
\underline{b}_{d}(\underline{s}) \leq 2 \underline{b}_{d}(s) \mathrm{e}^{\nu_{-}^{\prime \prime}(s-\underline{s}) / 4} \quad \text { for } s_{0} \leq \underline{s} \leq s .
$$

Using this in the integrand in (85), we arrive at

$$
\begin{aligned}
\left\|c_{d-}(s)\right\|_{2, t}(s) & \leq C_{0} \mathrm{e}^{-\nu_{-}^{\prime} s}+C_{0}^{\prime}|\lambda| \underline{b}_{d}(s) \\
& \leq C_{d} \underline{b}_{d}(s)\left(\underline{b}_{d}^{3}(s)+|\lambda|\right) .
\end{aligned}
$$

(In the second step above, we used (86) again to bound

$$
\left.\mathrm{e}^{-\nu_{-}^{\prime} s}=\left(\mathrm{e}^{-\nu_{-}^{\prime \prime} s / 4}\right)^{4} \leq C_{8} \underline{b}_{d}(s)^{4} \quad \text { for large } s .\right)
$$

Combining with (83), we obtain a similar estimate for $\left\|c_{d}(s)\right\|_{2, t}$ :

$$
\begin{aligned}
\left\|c_{d}(s)\right\|_{2, t}(s) & \leq C_{1} \mathrm{e}^{-\nu_{-}^{\prime} s}+C_{1}^{\prime}|\lambda| \underline{b}_{d}(s) \\
& \leq C_{d}^{\prime} \underline{b}_{d}(s)\left(\underline{b}_{d}^{3}(s)+|\lambda|\right) .
\end{aligned}
$$


We now return to verify the claim (86). To see this, note that projecting the flow equation to ker $A_{y}$, we have

$$
-b_{d}^{\prime}=\Pi_{\mathrm{ker} A_{y}}\left(\hat{n}_{(0, y)}\left(\lambda, \tilde{\zeta}_{\lambda}+c_{d}\right)-\hat{n}_{(0, y)}\left(\lambda, \tilde{\zeta}_{\lambda}(\infty)\right)\right) .
$$

Then the properties of $\hat{n}_{(0, y)}, \tilde{\zeta}_{\lambda}(\infty)$ and $u$ again give the estimate:

$$
\underline{b}_{d}^{\prime} \geq-\varepsilon^{\prime \prime}\left(\underline{b}_{d}+\left\|c_{d}\right\|_{2, t}\right)
$$

for a small positive constant $\varepsilon^{\prime \prime}$. Subtracting a small multiple of this from (84) and using (83), we have

$$
\left(\left\|c_{d-}\right\|_{2, t}-\varepsilon_{1} \underline{b}_{d}\right)^{\prime} \leq-\nu^{\prime \prime}\left(\left\|c_{d-}\right\|_{2, t}-\varepsilon_{1} \underline{b}_{d}\right) .
$$

Taking convolution product with the integral kernel of $d / d s+\nu^{\prime \prime}$, we have

$$
\left\|c_{d-}\right\|_{2, t} \leq \varepsilon_{1} \underline{b}_{d}+C_{1} e^{-\nu^{\prime \prime} s}
$$

Plugging this back in (83) and (88), we get

$$
\underline{b}_{d}^{\prime} \geq-\frac{\nu^{\prime \prime}}{4} \underline{b}_{d}-\varepsilon_{2} \mathrm{e}^{-\nu^{\prime \prime} s} .
$$

Now taking convolution product with the integral kernel of $d / d s+\nu^{\prime \prime} / 4$, we have for $\underline{s}<s$ :

$$
\begin{aligned}
& \underline{b}_{d}(s) \geq \underline{b}_{d}(\underline{s}) \mathrm{e}^{\nu^{\prime \prime}(\underline{s}-s) / 4}-\frac{4 \varepsilon_{2}}{3 \nu^{\prime \prime}}\left(\mathrm{e}^{-3 \nu^{\prime \prime}} \underline{s} / 4-\mathrm{e}^{-3 \nu^{\prime \prime} s / 4}\right) \mathrm{e}^{-\nu^{\prime \prime} s / 4} \\
& \geq \frac{1}{2} \underline{b}_{d}(\underline{s}) \mathrm{e}^{\nu^{\prime \prime}(\underline{s}-s) / 4} .
\end{aligned}
$$

To obtain the second inequality above, first use the first inequality and the fact that $s>\underline{s} \geq s_{0} \gg 1$ to obtain $\underline{b}_{d}(s) \geq C \mathrm{e}^{-\nu^{\prime \prime} s}$; then use this (with $s$ replaced by $\underline{s}$ ) to estimate

$$
\frac{4 \varepsilon_{2}}{3 \nu^{\prime \prime}}\left(\mathrm{e}^{-3 \nu^{\prime \prime} \underline{s} / 4}-\mathrm{e}^{-3 \nu^{\prime \prime} s / 4}\right) \leq \frac{\underline{b} d \underline{s})}{2} \mathrm{e}^{\nu^{\prime \prime} \underline{s} / 4} .
$$

Claim verified.

Next, to get estimates for higher derivatives of $c_{d}$ from (87), we need to elliptic bootstrap using (81) and apply Sobolev embedding as in the proof of Lemma I.5.1.7. To obtain the estimates claimed in the lemma, we need to bound the average of $\underline{b}_{d}$ in an interval about $s$ in terms of $\underline{b}_{d}(s)$. This is obtained using (86) and the fact that $\underline{b}_{d}$ is decreasing.

Next we compare $\tilde{w}(s)$ with the pregluing $w_{\chi}(s)$ to get a pointwise estimate of $\xi(s)$, as in Section 4.2.3. In this case, the first two formulas of (63) are still valid (with $\mathfrak{r}_{i}$ there replaced by $\mathfrak{r}_{\chi}$ ) by arguments similar to those in Section 4.2, but the third needs to be modified. In this region (where $\gamma \geq \mathfrak{r}_{\chi}$ ), we need to expand about $y_{\lambda}$ instead of $u_{\lambda}$ as in the proof of Lemma 5.2.1, keeping in mind that $\mu_{\lambda}$ is of order $\lambda^{1 / 2}$ while $\left(\mu_{\lambda}\right)_{\gamma}$ is of 
order $\lambda$. Recall that $\gamma_{\chi}$ satisfies the equation $\gamma_{\chi}^{\prime}=h\left(\gamma_{\chi}\right)$, with $h$ given by (73). The function $\tilde{\gamma}_{\chi}$ satisfies a similar equation:

$$
\tilde{\gamma}_{\chi}^{\prime}=h\left(\tilde{\gamma}_{\chi}\right)+\left\|\left(\mu_{\lambda}\right)_{\gamma}\left(\tilde{\gamma}_{\chi}\right)\right\|_{2, t}^{-2}\left\langle\left(\mu_{\lambda}\right)_{\gamma}\left(\tilde{\gamma}_{\chi}\right), E_{y_{\lambda}} T_{w_{\chi}, y_{\lambda}} \tilde{\xi}(s)+o\left(\|\tilde{\xi}(s)\|_{2,1, t}\right)\right\rangle_{2, t} .
$$

By (80) and (79), the absolute value of this can be bounded by

$$
\begin{array}{r}
C_{1}|\lambda|^{-1 / 2}\left(\left\|\mu_{\lambda}\left(\tilde{\gamma}_{\chi}\right)\right\|_{2,1, t}^{3}+|\lambda|\right)\left\|\mu_{\lambda}\left(\tilde{\gamma}_{\chi}\right)\right\|_{2,1, t} \\
\leq C_{2}|\lambda|^{3 / 2}\left(\breve{\gamma}_{\chi}-\tilde{\gamma}_{\chi}\right)\left(1+\lambda^{2}\left(\breve{\gamma}_{\chi}-\tilde{\gamma}_{\chi}\right)^{3}\right) .
\end{array}
$$

Recall also the estimate for $h$ from Section 5.2.1; we then obtain

$$
\begin{aligned}
\left|\Delta_{s}(\gamma)-\Delta_{s}\left(\mathfrak{r}_{\chi}\right)\right| & \leq C_{3} \int_{\mathfrak{r}_{\chi}}^{\gamma}\left|\frac{|\lambda|^{3 / 2}\left(\breve{\gamma}_{\chi}-\gamma\right)\left(1+\lambda^{2}\left(\breve{\gamma}_{\chi}-\gamma\right)^{3}\right)}{|\lambda|\left(\breve{\gamma}_{\chi}-\gamma\right)^{2}} d \gamma\right| \\
& \leq\left. C_{4}|\lambda|^{1 / 2}\left(\lambda^{2}\left(\breve{\gamma}_{\chi}-\gamma\right)^{2}+\left|\ln \left(\breve{\gamma}_{\chi}-\gamma\right)\right|\right)\right|_{\mathfrak{r}_{\chi}} ^{\gamma} .
\end{aligned}
$$

Using this and the facts that in this region

$$
\begin{gathered}
\left\|w_{\chi}^{\prime}\right\|_{2,2, t} \leq C_{5}|\lambda| \mathrm{e}^{-C_{6}|\lambda|^{1 / 2}\left(s-\gamma_{\chi}^{-1}\left(\mathfrak{r}_{\chi}\right)\right)} \quad \text { and } \\
\breve{\gamma}_{\chi}-\gamma_{\chi}(s) \leq C_{5}^{\prime}|\lambda|^{-1 / 2} \mathrm{e}^{-C_{6}|\lambda|^{1 / 2}\left(s-\gamma_{\chi}^{-1}\left(\mathfrak{r}_{\chi}\right)\right)},
\end{gathered}
$$

we can bound

$$
\left\|\tilde{\xi}_{\chi}\right\|_{2,2, t} \leq \varepsilon_{7}|\lambda| \mathrm{e}^{-C_{6}^{\prime}|\lambda|^{1 / 2}\left(s-\gamma_{\chi}^{-1}\left(\mathfrak{r}_{\chi}\right)\right)},
$$

where $\tilde{\xi}_{\chi}$ is defined by $\tilde{w}(s)=\exp \left(w_{\chi}(s), \tilde{\xi}_{\chi}(s)\right)$ as in Section 4.2. Recall also that $w(s)=\exp \left(w_{\chi}(s), \xi_{\chi}(s)\right)$. Combining the above estimate with (80) and the other two lines of $(63)$, we have

$$
\begin{aligned}
& \left\|\xi_{\chi}(s)\right\|_{2,2, t} \\
& \leq \begin{cases}\varepsilon_{7}|\lambda| \mathrm{e}^{-C_{6}^{\prime}|\lambda|^{1 / 2}\left(s-\gamma_{\chi}^{-1}\left(\mathfrak{r}_{\chi}\right)\right)} & \text { if } s \in\left[\gamma_{\chi}^{-1}\left(\mathfrak{r}_{\chi}\right), \infty\right), \\
\varepsilon_{8}|\lambda| & \text { if } s \text { is between } \gamma_{\chi}^{-1}\left(\mathfrak{r}_{\chi}\right) \text { and } \gamma_{\chi}^{-1}(R), \\
\varepsilon(\lambda) & \text { otherwise. }\end{cases}
\end{aligned}
$$

So over $\left(-\infty, \gamma_{\chi}^{-1}\left(\mathfrak{r}_{\chi}\right)\right] \times S^{1} \subset \Theta$, we can estimate $\left\|\xi_{\chi}\right\|_{W_{\chi}}$ by the same argument as in Section 4.2. The estimate over $\Theta_{y-}:=\left[\gamma_{\chi}^{-1}\left(\mathfrak{r}_{\chi}\right), \infty\right) \times S^{1}$ is replaced by the following. By (90),

$$
|\lambda|^{-1 / 2}\left\|\xi_{\chi}\right\|_{L^{p}\left(\Theta_{y-}\right)}+|\lambda|^{-1 / 2}\left\|\dot{\xi}_{\chi}\right\|_{L^{p}\left(\Theta_{y^{-}}\right)} \leq \varepsilon_{9}|\lambda|^{1 / 2-1 /(2 p)} \ll 1 .
$$

Next, to estimate $\xi_{\chi}^{\prime}$, it is equivalent to estimate $\xi_{\chi, y-}^{\prime}$, which is obtained by expanding the flow equation about $y_{\lambda_{-}}$. Here, we have an equation similar to (69), with $\bar{y}$ replaced by $\bar{y}_{\lambda_{-}}$, and $\alpha=0$. Using the error estimate in this region in the proof of Lemma 5.2.3, (89), Lemma I.5.3.2, we find

$$
|\lambda|^{-1 / 2}\left\|\left(\xi_{\chi, y_{-}}\right)_{T}^{\prime}\right\|_{L^{p}\left(\Theta_{y^{-}}\right)}|\lambda|^{-1}\left\|\left(\xi_{\chi, y_{-}}\right)_{L}^{\prime}\right\|_{L^{p}\left(\Theta_{y^{-}}\right)} \leq C_{9}|\lambda|^{1 / 2-1 /(2 p)} \ll 1 .
$$


This together with $(91)$ shows that $\left\|\xi_{\chi}\right\|_{W_{\chi}\left(\Theta_{y_{-}}\right)} \ll 1$. Now one may follow the argument in Section 1.2.7 to complete Step 4 of the proof of the gluing theorem.

5.3. When $u=\bar{y}$. We now assume that $x_{0}=z_{0}=y$, and $u=\bar{y}$, the constant flow at $y$.

5.3.1. The pregluing. Let $\underline{b}_{\lambda}^{0}(s)$ be the solution of

$$
-\left(\underline{b}_{\lambda}^{0}\right)^{\prime}=C_{y}^{\prime} \lambda+C_{y}\left(\underline{b}_{\lambda}^{0}\right)^{2}, \quad \text { with } \underline{b}_{\lambda}^{0}(0)=0,
$$

where $C_{y}, C_{y}^{\prime}$ are as defined in I.5.3.1. In other words, there are positive $\lambda$-independent constants $C_{0}, C^{\prime}$, such that

$$
\underline{b}_{\lambda}^{0}(s)=C_{0}|\lambda|^{1 / 2} \tanh \left(C^{\prime}|\lambda|^{1 / 2} s\right) .
$$

Let $b_{\lambda}^{0}(s):=\underline{b}_{\lambda}^{0}(s) \mathbf{e}_{y}$. Denote by $\underline{b}_{\lambda}^{0 \pm}=\lim _{s \rightarrow \pm \infty} \underline{b}_{\lambda}^{0}(s)= \pm C_{0}|\lambda|^{1 / 2}$. Let

$$
\tilde{\zeta}_{\lambda}:=b_{\lambda}^{0}+\beta_{+}\left(\eta_{\lambda+}-b_{\lambda}^{0+}\right)+\beta_{-}\left(\eta_{\lambda-}-b_{\lambda}^{0-}\right),
$$

where $\eta_{\lambda \pm}$ are defined by $\exp \left(y, \eta_{\lambda_{ \pm}}\right)=y_{\lambda_{ \pm}}$, and $\beta_{-}(s):=\beta\left(|\lambda|^{-1}+s\right)$; $\beta_{+}(s):=\beta\left(|\lambda|^{-1}-s\right)$.

We define the pregluing $w_{\lambda}$ in this case by

$$
w_{\lambda}:=\exp \left(y, \tilde{\zeta}_{\lambda}\right)
$$

5.3.2. The weighted norms. Recall the definition of $W_{y-}, L_{y-}$ from Section 5.2.4. Let $W_{y+}, L_{y+}$ and $W_{y}, L_{y}$ be similarly defined for elements in $\Gamma\left(\bar{y}_{\lambda+}^{*} K\right)$ and $\Gamma\left(\bar{y}^{*} K\right)$, respectively, with longitudinal directions given by $\mathbf{e}_{y_{\lambda+}}$ and $\mathbf{e}_{y}$.

Via the map $T_{y, w_{\lambda}}: \Gamma\left(\bar{y}^{*} K\right) \rightarrow \Gamma\left(\left(w_{\lambda}\right)^{*} K\right)$, the norms $W_{y}, L_{y}$ on $\Gamma\left(\bar{y}^{*} K\right)$ induce norms on $\Gamma\left(\left(w_{\lambda}\right)^{*} K\right)$, which we denote by $W_{\lambda}, L_{\lambda}$. The associated spaces shall be the domain and range for $E_{w_{\lambda}}$.

By the estimates for $\eta_{\lambda \pm}$, it is easy to see that the induced norms on $\Gamma\left(\left(w_{\lambda}\right)^{*} K\right)$ via $T_{y_{\lambda \pm}, w_{\lambda}}$ from $W_{y \pm}, L_{y \pm}$ are commensurate with $W_{\lambda}, L_{\lambda}$.

5.3.3. Error estimates. Divide $\Theta$ into three regions: $\Theta_{a}, \Theta_{b}$, and $\Theta_{c}$ corresponding to $s<-|\lambda|,|s|<|\lambda|$, and $s>|\lambda|$, respectively. We will expand $\bar{\partial}_{J X_{\lambda}} w_{\lambda}$ around $y_{\lambda+}, y, y_{\lambda-}$, respectively, in the three regions.

Over $\Theta_{b}$, using (92) and the fact of $y$ being in a standard d-b neighborhood, we have

$$
\begin{aligned}
& \left(T_{y, w_{\lambda}}\right)^{-1} \bar{\partial}_{J X_{\lambda}} w_{\lambda} \\
= & E_{y} \tilde{\zeta}_{\lambda}+n_{y}\left(\tilde{\zeta}_{\lambda}\right)+\left(T_{y, w_{\lambda}}\right)^{-1} \delta_{\lambda} \mathcal{V}\left(w_{\lambda}\right) \\
=- & \beta_{+}^{\prime}\left(\eta_{\lambda+}-b_{\lambda}^{0+}\right)+\beta_{-}^{\prime}\left(\eta_{\lambda-}-b_{\lambda}^{0-}\right)+\beta_{+} A_{y}\left(\eta_{\lambda+}-b_{\lambda}^{0+}\right) \\
& \quad+\beta_{-} A_{y}\left(\eta_{\lambda-}-b_{\lambda}^{0-}\right)+C_{y}\left\langle\mathbf{e}_{y}, 2 b_{\lambda}^{0}+\delta\right\rangle_{2, t} \delta+\Pi_{\mathbf{e}_{y}}^{\perp} O\left(\left(\left\|\tilde{\zeta}_{\lambda}\right\|_{2,1, t}+|\lambda|\right)^{2}\right),
\end{aligned}
$$

where $\delta:=\beta_{+}\left(\eta_{\lambda+}-b_{\lambda}^{0+}\right)+\beta_{-}\left(\eta_{\lambda-}-b_{\lambda}^{0-}\right)$. 
From the estimates for $\eta_{\lambda \pm}$ in the proof of Lemma I.5.3.2, one sees that

$$
|\lambda|^{-1}\left\|\delta_{L}\right\|_{2,1, t}+|\lambda|^{-1 / 2}\left\|\delta_{T}\right\|_{2,1, t} \leq C|\lambda|^{1 / 2} \quad \forall s,
$$

and therefore from (94)

$$
\left\|\bar{\partial}_{J X_{\lambda}}\left(w_{\lambda}\right)\right\|_{L_{\lambda}\left(\Theta_{b}\right)} \leq C|\lambda|^{1 / 2-1 / p} .
$$

The estimates on $\Theta_{a}$ and $\Theta_{c}$ are similar, so we shall focus on $\Theta_{c}$. In this region, writing $w_{\lambda}(s)=\exp \left(y_{\lambda+}, \mu_{\lambda+}(s)\right)$, we have

$$
\left(T_{y, w_{\lambda}}\right)^{-1} \bar{\partial}_{J X_{\lambda}} w_{\lambda}=E_{y_{\lambda+}} \mu_{\lambda+}+n_{y_{\lambda+}}\left(\mu_{\lambda+}\right) .
$$

From the definition of $\mu_{\lambda+},\left\|\mu_{\lambda+}(s)\right\|_{p, 1, t} \leq C\left\|b_{\lambda}^{0+}-b_{\lambda}^{0}(s)\right\|_{p, 1, t}$. So by (93), (96),

$$
\left\|\bar{\partial}_{J X_{\lambda}}\left(w_{\lambda}\right)\right\|_{L_{\lambda}\left(\Theta_{c}\right)} \leq C|\lambda|^{-1 / 2-1 / p} \mathrm{e}^{-C^{\prime}|\lambda|^{-1}} \rightarrow 0 \quad \text { as } \lambda \rightarrow 0 .
$$

5.3.4. Existence and uniform boundedness of right inverse to $E_{w_{\lambda}}: W_{\lambda} \rightarrow L_{\lambda}$. In this case, let

$$
W_{\lambda}^{\prime}:=\left\{\xi \in W_{\lambda} \mid \xi_{L}(0)=0\right\} .
$$

Again assume the existence of a sequence $\left\{\xi_{\lambda} \in W_{\lambda}^{\prime}\right\}_{\lambda}$ satisfying (74), with the obvious modification.

Divide $\Theta$ into three regions $\Theta_{y}, \Theta_{y \pm}$ corresponding, respectively, to the three possibilities: $|s| \leq|\lambda|^{-1 / 2}, \pm s \geq|\lambda|^{-1 / 2}$. Let $\Theta_{y}^{\prime} \supset \Theta_{y}$ be the region in which $|s|<(1+\varepsilon)|\lambda|^{-1 / 2}$; let $\Theta_{y \pm}^{\prime} \supset \Theta_{y \pm}$ be the region in which $\pm s>$ $(1-\varepsilon)|\lambda|^{-1 / 2}$, where $1 \gg \varepsilon>0$. Instead of estimating $\left\|\xi_{\lambda}\right\|_{W_{\lambda}}$, we shall estimate

$$
\xi_{\lambda, y}:=T_{w_{\lambda}, y} \xi_{\lambda}, \quad \xi_{\lambda, y \pm}:=T_{w_{\lambda}, y \pm} \xi_{\lambda}
$$

in $W_{y}$ or $W_{y \pm}$-norm over $\Theta_{y}$ and $\Theta_{y \pm}$, respectively. First, observe the following analog of Lemma 5.2.4 over $\Theta_{y}$ and $\Theta_{y \pm}$.

Lemma (Analog of Floer's lemma). Let $\xi_{\lambda}$ be as in (74) with $W_{\chi}, W_{\chi}^{\prime}, L_{\chi}$ replaced by $W_{\lambda}, W_{\lambda}^{\prime}, L_{\lambda}$ respectively. Then for all sufficiently small $\lambda$, there is a small positive number, $\varepsilon_{0}(\lambda), \lim _{\lambda \rightarrow 0} \varepsilon_{0}(\lambda)=0$, such that

$$
\begin{aligned}
& (a)|\lambda|^{1 /(2 p)}\left\|\xi_{\lambda, y}\right\|_{L^{\infty}\left(\Theta_{y}^{\prime}\right)}+\left\|\left(\xi_{\lambda, y}\right)_{L}\right\|_{L^{\infty}\left(\Theta_{y}^{\prime}\right)} \leq \varepsilon_{0}(\lambda)|\lambda|^{1 / 2+1 /(2 p)} ; \\
& (b)|\lambda|^{1 /(2 p)}\left\|\xi_{\lambda, y \pm}\right\|_{L^{\infty}\left(\Theta_{y \pm}^{\prime}\right)}+\left\|\left(\xi_{\lambda, y \pm}\right)_{L}\right\|_{L^{\infty}\left(\Theta_{y \pm}^{\prime}\right)} \leq \varepsilon_{0}(\lambda)|\lambda|^{1 / 2+1 /(2 p)} .
\end{aligned}
$$

Proof. The $L_{t}^{\infty}$-estimate for $\xi_{\lambda}$ (and hence $\xi_{\lambda, y}$ and $\xi_{\lambda, y \pm}$ ) is now routine. The estimates for the longitudinal components follow the rescaling argument in the proof of Lemma 5.2.4, with the following modifications.

On $\Theta_{y}^{\prime}$, one may similarly define a sequence $\tilde{\varsigma}_{\lambda}$ of $L_{1}^{p}$-bounded functions on $[-1,1]$, which converges to $\tilde{\varsigma}_{0}$ which satisfy also an equation of the form (76), but now $\chi \sim C \tanh \left(C^{\prime} \tau\right)$. Because $\left(\xi_{\lambda}\right)_{L}(0)=0, \tilde{\varsigma}_{0}(0)=0$, and thus $\tilde{\varsigma}_{0}=0$. This proves part (a) above. 
On $\Theta_{y \pm}^{\prime}$, we have another version of $\tilde{\varsigma}_{\lambda}$ and $\tilde{\varsigma}_{0}$ (which are now functions on $[1, \infty),(-\infty,-1]$, respectively), and the argument in the proof of Lemma 5.2.4 again gives a bound on $\left\|\tilde{\varsigma}_{0}\right\|_{\infty}$ by $\left|\tilde{\varsigma}_{0}\right|( \pm 1)$, which vanishes by the estimate for $\left\|\left(\xi_{\lambda}\right)_{L}\right\|_{L^{\infty}\left(\Theta_{y}^{\prime}\right)}$ obtained in part (a). This proves part (b).

We now return to estimate $\left\|\xi_{\lambda, y}\right\|_{W_{y}}\left(\Theta_{y}\right)$ and $\left\|\xi_{\lambda, y \pm}\right\|_{W_{y \pm}}\left(\Theta_{y \pm}\right)$.

On $\Theta_{y}^{\prime}$ : let $\chi_{y}$ be a smooth cutoff function which vanishes outside $(-1,1)$ and let $\beta_{y}(s):=\chi_{y}\left(s /|\lambda|^{-1 / 2}\right)$. Since $\left(\xi_{\lambda, y}\right)_{L}(0)=0$, applying Lemma 3.3.3 (c) to the longitudinal component, one may estimate:

$$
\begin{aligned}
\left\|\beta_{y} \xi_{\lambda, y}\right\|_{W_{y}\left(\Theta_{y}\right) \leq} & C\left\|E_{\bar{y}}\left(\beta_{y} \xi_{\lambda, y}\right)\right\|_{L_{\lambda}\left(\Theta_{y}^{\prime}\right)} \\
\leq & C_{1}\left\|E_{w_{\lambda}} \xi_{\lambda}\right\|_{L_{\lambda}\left(\Theta_{y}^{\prime}\right)}+C_{2}\left(|\lambda|^{1 / 2}\left\|\beta_{y} \xi_{\lambda}\right\|_{W_{\lambda}\left(\Theta_{y}^{\prime}\right)}\right. \\
& \left.\quad+\left\|\beta_{y}\left(\xi_{\lambda, y}\right)_{L}\right\|_{W_{y}\left(\Theta_{y}^{\prime}\right)}\right)+C_{3}\left\|\beta_{y}^{\prime} \xi_{\lambda}\right\|_{L_{\lambda}\left(\Theta_{y}^{\prime}\right)} \\
\leq & C_{1} \varepsilon_{E}+C_{4}\left(|\lambda|^{1 / 2}+\varepsilon_{0}\right) .
\end{aligned}
$$

In the above, the second term in the penultimate line came from the difference between $E_{w_{\chi}}$ and the conjugate of $E_{y}$, using the fact that $\left\|\tilde{\zeta}_{\lambda}\right\|_{\infty, 1, t} \leq$ $C|\lambda|^{1 / 2}$. The last line used Lemma 5.3.4 (a) and the equation for $\left(\xi_{\lambda, y}\right)_{L}$.

On $\Theta_{y \pm}^{\prime}$ one may estimate similarly. Let $\beta_{y \pm}$ be smooth cutoff functions supported on $\Theta_{y \pm}^{\prime}$ with value 1 over $\Theta_{y \pm}$ and $\left|\beta_{y \pm}^{\prime}\right| \leq C|\lambda|^{1 / 2}$. By the eigenvalue estimate for $A_{y_{\lambda \pm}}$ in I.5.3.2,

$$
\left\|\beta_{y \pm} \xi_{\lambda, y \pm}\right\|_{W_{y \pm}} \leq C\left\|E_{y_{\lambda \pm}}\left(\beta_{y \pm} \xi_{\lambda, y \pm}\right)\right\|_{L_{ \pm}} .
$$

The RHS can be estimated like (77), (78) using Lemma 5.3.4 (b) below.

Finally, from the estimates for $\xi_{\lambda, y}$ and $\xi_{\lambda, y \pm}$ above, we obtain the desired contradiction that $\left\|\xi_{\lambda}\right\|_{W_{\lambda}} \ll 1$.

5.3.5. Surjectivity of the gluing map. We have the routine estimate for the nonlinear term to define the gluing map. The main isssue is again to show that the gluing map surjects to a neighborhood of $\mathbb{S}$ in the parameterized moduli space of broken trajectories.

Let $(\lambda, \hat{w}) \in \hat{\mathcal{M}}_{P}^{\Lambda, 1}\left(\mathbf{y}_{+}, \mathbf{y}_{-}\right)$be in a chain-topology neighborhood of $\bar{y} \in$ $\hat{\mathcal{M}}_{P}^{\Lambda, 1,+}$. Choose a representative $w$ of $\hat{w}$ such that writing

$$
w(s)=\exp (y, \zeta(s)), \quad w_{\lambda}(s)=\exp (y, \tilde{\zeta}(s)),
$$

the difference $\eta:=\zeta-\tilde{\zeta}$ satisfies $\eta_{L}(0)=0$. Writing $w(s)=\exp \left(w_{\lambda}(s), \xi(s)\right)$, we want to show that $\|\xi\|_{W_{\lambda}} \ll 1$; equivalently, it suffices to estimate $\eta$.

Let $\epsilon^{\prime} \gg|\lambda|^{1 / 2}$ be a small positive number. Consider the three regions $\Theta_{a}^{\epsilon^{\prime}}=\left(-\infty,-\epsilon^{\prime}|\lambda|^{-1}\right] \times S^{1}, \Theta_{b}^{\epsilon^{\prime}}=\left[-\epsilon^{\prime}|\lambda|^{-1}, \epsilon^{\prime}|\lambda|^{-1}\right] \times S^{1}, \Theta_{c}^{\epsilon^{\prime}}=\left[\epsilon^{\prime}|\lambda|^{-1}, \infty\right) \times$ $S^{1}$ separately. 
From the flow equation and the definition of $w_{\lambda}$, we find that $\eta_{L}, \eta_{T}$ satisfy, respectively:

$$
\begin{gathered}
\underline{\eta}_{L}^{\prime}+C_{y}\left(\underline{\tilde{\zeta}}_{L}+\underline{\zeta}_{L}\right) \underline{\eta}_{L}=O\left(\left(|\lambda|+\left\|\eta_{T}(s)\right\|_{2,1, t}\right)^{2}+\left\|\zeta_{L}(s)\right\|_{2,1, t}^{4}\right) \\
\eta_{T}^{\prime}(s)+A_{y} \eta_{T}(s)=O\left(\left(|\lambda|+\|\zeta\|_{2,1, t}\right)^{2}\right) .
\end{gathered}
$$

(In the usual notation, $\eta_{L}=: \underline{\eta}_{L} \mathbf{e}_{y} ; \zeta_{L}=: \underline{\zeta}_{L} \mathbf{e}_{y}$.)

Equation (99) and the fact that $\eta(\infty)=\eta(-\infty)=0$ imply:

$$
\left\|\eta_{T}\right\|_{2,2, t} \leq C_{1}\left(|\lambda|+\left\|\zeta_{L}\right\|_{\infty}\right)^{2} \quad \forall s .
$$

The argument to get this estimate should be by-now familiar to the reader (cf., e.g., the proof of Lemma 5.2.5): Take $L_{t}^{2}$-inner product of (99) with $\eta_{T-\text {, }}$ $\eta_{T+}$, respectively, and integrate over $s$, one obtains

$$
\left\|\eta_{T}\right\|_{2} \leq C_{2}\left(|\lambda|+\sup _{s}\left\|\zeta_{L}\right\|_{2, t}(s)\right)^{2} .
$$

Then apply the usual elliptic bootstrapping and Sobolev embedding to get estimates on higher derivatives. Finally, observe that on the 1-dimensional subspace of longitudal direction, the various norms are all commensurate.

On the other hand, $\zeta_{L}$ satisfies

$$
\underline{\zeta}_{L}^{\prime}+C_{y} \underline{\zeta}_{L}^{2}+C_{y}^{\prime} \lambda=O\left(\left(|\lambda|+\left\|\eta_{T}\right\|_{2,1, t}+\left\|\delta_{T}\right\|_{2,1, t}+\|\zeta\|_{2,1, t}^{2}\right)^{2}\right),
$$

with $\zeta_{L}^{\prime}(\infty)=0=\zeta_{L}^{\prime}(-\infty)$ (so when $\left|\underline{\zeta}_{L}\right|(s)$ reaches maximum, $\underline{\zeta}_{L}^{\prime}=0$ ). Combining this with (95) and (100), we have

$$
\left|\zeta_{L}\right|_{\infty} \leq C_{L}|\lambda|^{1 / 2}
$$

Plugging this back in (100), we get

$$
\left\|\eta_{T}\right\|_{2,2, t} \leq C_{1}|\lambda| \text {. }
$$

Using these $L^{\infty}$ estimates for $\eta_{T}, \zeta_{L}$ and multiplying (98) with $\underline{\eta}_{L}$, we obtain

$$
-C^{\prime}|\lambda|^{2} \leq\left|\underline{\eta}_{L}\right|^{\prime}+C_{y}\left(\underline{\tilde{\zeta}}_{L}+\underline{\zeta}_{L}\right)\left|\underline{\eta}_{L}\right| \leq C|\lambda|^{2} .
$$

Now since $\underline{\tilde{\zeta}}_{L}, \underline{\zeta}_{L}$ are both $\geq 0$ when $s \geq 0$, and are both $\leq 0$ when $s \leq 0$, we see that

$$
\begin{aligned}
\left|\underline{\eta}_{L}\right|^{\prime} \leq C|\lambda|^{2} \quad \text { when } s \geq 0 ; \\
-\left|\underline{\eta}_{L}\right|^{\prime} \leq C|\lambda|^{2} \quad \text { when } s \leq 0 .
\end{aligned}
$$

Integrating using the initial condition that $\eta_{L}(0)=0$, we see that

$$
\left\|\eta_{L}\right\|_{L^{\infty}\left(\Theta_{b}^{\epsilon^{\prime}}\right)} \leq C_{1} \epsilon^{\prime}|\lambda| .
$$

Combining this with (101), we get

$$
\|\xi\|_{W_{\lambda}\left(\Theta_{b}^{\epsilon^{\prime}}\right)} \leq C_{2} \epsilon^{1+1 /(2 p)}|\lambda|^{1 / 2-1 / p} \rightarrow 0 \quad \text { as } \lambda \rightarrow 0 .
$$


We now turn to estimating $\xi$ over $\Theta_{a}^{\epsilon^{\prime}}$ and $\Theta_{c}^{\epsilon^{\prime}}$. We shall only consider $\Theta_{c}^{\epsilon^{\prime}}$ since the other works by analogy. On this region, writing $w_{\lambda}(s)=$ $\exp \left(y_{\lambda_{-}}, \tilde{\zeta}_{\lambda-}(s)\right)$, we have from the definition of $w_{\lambda}$ that

$$
\left\|\tilde{\zeta}_{\lambda-}\right\|_{2,2, t}(s) \leq C_{3}|\lambda|^{3 / 2} \mathrm{e}^{-C_{6}|\lambda|^{1 / 2}\left(s-\epsilon^{\prime}|\lambda|^{-1}\right)}+\text { terms involving } \delta .
$$

We may ignore the terms involving $\delta$ since by (95), its contribution to the $W$-norm is at most of order $|\lambda|^{1 / 2}$. On the other hand, by standard estimates $w(s)$ has the same exponential decay behavior in this region, and so combining the estimate for $\zeta_{\lambda_{-}}\left(\epsilon^{\prime}|\lambda|^{-1}\right)$ above and (101), (102), we have on this region

$$
\left\|\zeta_{\lambda-}\right\|_{2,2, t}(s) \leq C_{4} \epsilon^{\prime}|\lambda| \mathrm{e}^{-C_{6}^{\prime}|\lambda|^{1 / 2}\left(s-\epsilon^{\prime}|\lambda|^{-1}\right)},
$$

where $\zeta_{\lambda_{-}}$is defined by $\exp \left(y_{\lambda_{-}}, \zeta_{\lambda_{-}}\right)=w(s)$. Together with the previous expression, we obtain a pointwise estimate for $\xi(s)$ on this region, which, when combined with (98), (99), yields

$$
\|\xi\|_{W_{\lambda}\left(\Theta_{c}^{\epsilon^{\prime}}\right)} \leq C_{5} \epsilon^{\prime}|\lambda|^{1 / 2-1 /(2 p)} \ll 1 .
$$

\section{The handleslide bifurcation}

The purpose of this section is to verify the bifurcation behavior at handleslides predicted in I.4.3, namely, Propositions 6.1.1, 6.1.2 below.

6.1. Summary of results. Combined with the previous gluing theorems: Propositions 2.1, 5.1, the following proposition completes the verification of (RHFS2c), (RHFS3c) for admissible $(J, X)$-homotopies.

6.1.1. Proposition. Let $\left(J^{\Lambda}, X^{\Lambda}\right)$ be an admissible $(J, X)$-homotopy connecting two regular pairs, and $\mathbf{x}, \mathbf{z}$ be two path components of $\mathcal{P}^{\Lambda} \backslash \mathcal{P}^{\Lambda, \operatorname{deg}}$. Then:

(a) a chain-topology neighborhood of $\mathbb{T}_{P, \text { hs-s }}(\mathbf{x}, \mathbf{z} ; \Re)$ in $\hat{\mathcal{M}}_{P}^{\Lambda, 1,+}\left(\mathbf{x}, \mathbf{z} ;\right.$ wt_y,ep $\left._{-} \leq \Re\right)$ is l.m.b. along $\mathbb{T}_{P, \mathrm{hs}-\mathrm{s}}(\mathbf{x}, \mathbf{z} ; \Re) ;$

(b) a chain-topology neighborhood of $\mathbb{T}_{O, \mathrm{hs}-\mathrm{s}}(\Re)$ in $\hat{\mathcal{M}}_{O}^{\Lambda, 1,+}\left(\mathrm{wt}_{-y_{\text {,ep }}} \leq \Re\right)$ is l.m.b. along $\mathbb{T}_{O, \mathrm{hs}-\mathrm{s}}(\Re)$.

The proof follows the standard gluing construction outlined in Section 1.2, and shall be omitted. A description of the relevant K-models will be given in Section 7.3.2 and 7.3.3. A result analogous to part (a) above is also given by Proposition 4.2 of [6].

The rest of this section will be devoted to the proof of Proposition 6.1.2.

6.1.2. Proposition. Let $\left(J^{\Lambda}, X^{\Lambda}\right)$ be an admissible $(J, X)$-homotopy, and $u \in \hat{\mathcal{M}}_{P}^{\Lambda, 0}(\mathbf{x}, \mathbf{x})$. Then $(N E P)$ holds for $u$.

Without loss of generality, we restrict our attention to a $J \mid X$-homotopy without death-birth bifurcations throughout this section. 
6.2. Nonequivariant perturbations on finite-cyclic covers. This subsection contains the main body of the proof of Proposition 6.1.2. We first discuss a simpler situation in which the nonequivariant perturbation may be obtained from a vector field on a finite-cyclic covering of $M$. In general, we need to resort to nonlocal perturbations.

6.2.1. A special case: local perturbations from finite-cyclic covers of $M$. If a finite-cyclic cover $\mathrm{C}^{\nu, m} \rightarrow \mathcal{C}$ is (a path component of) the pullback bundle of a finite-cyclic cover $\hat{M} \rightarrow M$ via $e_{f}: \mathcal{C} \rightarrow M$ (cf. I.3.1.1), then a nonequivariant function or vector field on $\hat{M}$ may induce a nonequivariant function or vector field on $\mathrm{C}^{\nu e, m}$.

Example. Assume the conditions of Corollary I.2.2.5 (namely, $M$ is monotone, $f$ is symplectic isotopic to id, and $\gamma_{0}$ is the trace of a point under the symplectic isotopy). We claim that in this case, for any $m \in \mathbb{Z}^{+}$not dividing $\operatorname{div}([u])$, there exists a $u$-breaking $m$-cyclic cover of $\mathcal{C}$ via the above pull-back construction. Thus, in this case, Proposition 6.1.2 may be proven by simply repeating the argument for Proposition I.6.2.2 for nonequivariant Hamiltonian perturbations over finite-cyclic covers of $M$. (In fact, only Lemma I.6.2.5 needs to be redone.)

To see the claim, recall that in this case,

$$
\mathfrak{H}=H_{1}(\mathcal{C} ; \mathbb{Z})=\pi_{2}(M) \oplus H_{1}(M ; \mathbb{Z}), \quad \text { and } \quad e_{f *}=0 \oplus \mathrm{id}
$$

with respect to this decomposition. Notice that $e_{f *}([u])$ is a nontorsion element in $H_{1}(M ; \mathbb{Z})$. Otherwise, by the commutative diagram from I.(12),

$$
k[u]=\left.b \in \operatorname{ker} c_{1}\right|_{\pi_{2}(M)} \quad \text { for some } k \in \mathbb{Z}^{+} .
$$

But then by monotonicity of $M$,

$$
\left[y_{X}\right](k[u])=\omega(b)-e_{f}^{*} \theta_{X}(k[u])=0,
$$

contradicting the fact that $u$ has positive energy.

Thus, for any $m \in \mathbb{Z}^{+}$not dividing $\operatorname{div}([u])$, one may simply set $\nu_{M} \in H^{1}(M ; \mathbb{Z})$ to be a primitive class with $\nu_{M}\left(e_{f *}([u])\right)=\operatorname{div}([u])$ and take $\mathrm{e}^{\nu, m}=e_{f}^{*} M^{\nu_{M}, m}$. Furthermore, such a finite-cyclic cover is always $\mathfrak{H}$-adapted, and $u$-breaking if $m$ does not divide $\operatorname{div}([u])$.

However, this simple construction does not give all the $u$-breaking finite covers we need.

6.2.2. The general case: nonlocal perturbations. Let $\mathcal{C}^{\nu, m}$ be a $u$ breaking, $\mathfrak{H}$-adapted $m$-cyclic cover of $\mathcal{C}$ introduced in I.4.4.5.

We shall often make use of the following convenient description of $\mathfrak{e}^{\nu, m}$ :

$$
\mathfrak{e}^{\nu, m}=\left\{(z,[\mu]) \mid z \in \mathcal{C}, \mu:[0,1] \rightarrow \mathcal{C} ; \mu(0)=\gamma_{0}, \mu(1)=z\right\} / \sim,
$$


where $\left(z_{1},\left[\mu_{1}\right]\right) \sim\left(z_{2},\left[\mu_{2}\right]\right)$ iff $z_{1}=z_{2}$ and $\nu\left(\left[\mu_{1}-\mu_{2}\right]\right)=0 \bmod m$. Such an equivalence class shall be denoted by a pair $\left(z,[\mu]_{m}\right)$.

Recall that $\nu \in \operatorname{Hom}(\mathfrak{H}, \mathbb{Z})$. The fact that $\mathrm{e}^{\nu, m}$ is $u$-breaking implies that $\nu$ is nontorsion. Thus, one may find a class $\nu_{2} \in H^{2}\left(T_{f} ; \mathbb{R}\right)$ extending $\nu$ by linearity, that is, satisfying $\nu_{2}\left(\left(i_{\mathfrak{H}} \operatorname{ker} \nu\right) \otimes \mathbb{R}\right)=0$, and $\nu_{2}\left(i_{\mathfrak{H}}[u]\right)=\operatorname{div}(u)$ where $i_{\mathfrak{H}}: \mathfrak{H} \hookrightarrow H_{2}\left(T_{f} ; \mathbb{Z}\right)$ is the inclusion. Let $\omega_{\nu}$ be a smooth closed 2-form on $T_{f}$ in the cohomology class $\nu_{2}$.

The 2 -form $\omega_{\nu}$ defines an $\mathbb{R}$-valued function $\Omega_{\nu}$ on $\tilde{\mathcal{C}}$, by setting

$$
\Omega_{\nu}(z,[\mu]):=\int_{[0,1] \times S_{1}^{1}} \mu^{*} \omega_{\nu}
$$

This induces an $\mathbb{R} / m \mathbb{Z}$-valued function on $\mathrm{C}^{\nu, m}$, which we shall denote by the same notation.

Definition (A class of nonlocal perturbations). Let $\chi: \mathbb{R} / m \mathbb{Z} \rightarrow \mathbb{R}$ be a smooth function, and let $P \in \mathcal{H}$. We define the formal vector field $\wp_{\chi} P$ on $\mathrm{e}^{\nu, m}$ by

$$
\wp_{\chi P}\left(z,[\mu]_{m}\right):=\chi\left(\Omega_{\nu}\left(z,[\mu]_{m}\right)\right) \nabla P(z) .
$$

For a path $\left(u(s),[\mu(s)]_{m}\right)$ in $\mathrm{C}^{\nu, m}$, let

$$
\bar{\partial}_{J X}^{\chi P}\left(u,[\mu]_{m}\right):=\bar{\partial}_{J X} u+\wp_{\chi P}\left(u,[\mu]_{m}\right) .
$$

Similarly, for a smooth function $\chi^{\Lambda}: \Lambda \times \mathbb{R} / m \mathbb{Z} \rightarrow \mathbb{R}$ and $P^{\Lambda} \in \mathcal{H}^{\Lambda}$, one may define a path of formal vector fields $\left\{\wp_{\chi_{\lambda} P_{\lambda}}\right\}_{\lambda \in \Lambda}$ and the section $\bar{\partial}_{J^{\Lambda} X^{\Lambda}}^{\chi^{\Lambda} P^{\Lambda}}$ on $\mathcal{B}_{P}^{\Lambda}$ or $\mathcal{B}_{O}^{\Lambda}$.

For the rest of this section, a " $\chi P$-perturbed flow" or simply a "perturbed flow" will refer to a solution of $\bar{\partial}_{J X}^{\chi P}\left(u,[\mu]_{m}\right)=0$. One may define the moduli spaces of such flows, $\mathcal{M}_{P ; \nu, m}(J, X ; \chi, P), \mathcal{M}_{O ; \nu, m}(J, X ; \chi, P)$, etc., and their parameterized versions, in the usual manner (cf. I.2.1.2, I.4.3.1). Notice that if one chooses $P \in V_{\delta}^{k}(J, X)$ and $P^{\Lambda} \in V_{\delta}^{\Lambda ; k, \kappa}\left(J^{\Lambda}, X^{\Lambda}\right)$, then

$$
\mathcal{P}(X ; \chi, P)=\mathcal{P}(X) ; \quad \mathcal{P}^{\Lambda}\left(X^{\Lambda} ; \chi^{\Lambda}, P^{\Lambda}\right)=\mathcal{P}^{\Lambda}\left(X^{\Lambda}\right),
$$

and in both equalities, the former is nondegenerate iff the latter is. We shall show in the next subsection that in this case, when $\chi, \chi^{\Lambda}$ are sufficiently small, and if $(J, X)$ is regular and $\left(J^{\Lambda}, X^{\Lambda}\right)$ admissible, then the moduli spaces of $\chi P$-perturbed flows and their parameterized versions satisfy all the usually expected regularity and compactness properties, as described by (FS2), (FS3) and (RHFS2*), (RHFS3*).

Proof of Proposition 6.1.2. Let $\Re \in \mathbb{R}^{+}$and $\mathrm{C}^{\nu, m}$ be fixed as in the statement of (NEP). Without loss of generality, assume $\Pi_{\Lambda} u=0$.

The admissible $(J, X)$-homotopy $\left(J^{\Lambda}, X^{\Lambda}\right)$ induces a homotopy of formal flows on $\mathcal{C}^{\nu, m}$, which satisfies all the properties listed in I.6.2.3 for admissibility, except for Property (8) (injectivity of $\left.\Pi_{\Lambda}\right|_{\hat{\mathfrak{M}}_{P}^{\Lambda, 0}}$ ): at $\lambda=0$, there are 
$m$ distinct elements in $\hat{\mathcal{M}}_{P}^{0}\left(J_{\lambda}, X_{\lambda}\right)$, which are precisely the $m$ different lifts of $u$.

We write this induced homotopy of vector fields as $\left\{\mathcal{V}^{\nu, m}\left(J_{\lambda}, X_{\lambda}\right)\right\}_{\lambda \in \Lambda}$.

To achieve Property (8), we shall consider homotopy of vector fields on $\mathrm{e}^{\nu, m}$ of the form

$$
\left\{\mathcal{V}^{\nu, m}\left(J_{\lambda}, X_{\lambda} ; \chi_{\lambda}, P_{\lambda}\right)\right\}_{\lambda \in \Lambda}:=\left\{\mathcal{V}^{\nu, m}\left(J_{\lambda}, X_{\lambda}\right)+\wp_{\chi_{\lambda}} P_{\lambda}\right\}_{\lambda \in \Lambda},
$$

where

$$
P^{\Lambda} \in V_{\delta}^{\Lambda ; k, \kappa}\left(J^{\Lambda}, X^{\Lambda}\right) .
$$

In fact, since $\hat{\mathcal{M}}_{P}^{\Lambda, 0}\left(J^{\Lambda}, X^{\Lambda} ; \mathrm{wt}_{-}\langle y\rangle, e_{\mathcal{P}} \leq \Re\right)$ consists of finitely many points, each projecting under $\Pi_{\Lambda}$ to distinct values, we may assume that

$$
P_{\lambda}=0 \quad \text { for } \lambda \in \Lambda \backslash S,
$$

where $S$ is a small interval about $\Pi_{\Lambda}(u)=0$, so that

$$
S \cap\left(\Lambda_{\mathrm{db}} \cup \Pi_{\Lambda}\left(\hat{\mathcal{M}}_{P}^{\Lambda, 0}\left(J^{\Lambda}, X^{\Lambda} ; \mathrm{wt}_{-\langle y\rangle, e_{\mathcal{P}}} \leq \Re\right) \backslash\{u\}\right)=\emptyset .\right.
$$

Such perturbed homotopy of formal flows might no longer be co-directional; however, Properties (1)-(6) of I.6.2.3 are preserved. Moreover, we shall see in the next subsection that as long as $\chi$ is sufficiently small in $C_{\epsilon}$-norm, the parameterized moduli spaces remain $\Re$-regular (i.e., $\Re$-truncated version of I.6.2.3 (7) holds).

We now describe an explicit choice of $\chi^{\Lambda}, P^{\Lambda}$ among all those satisfying both (104), (105), so that I.6.2.3 (8) may be achieved. For this purpose, the argument in the proof of Lemma I.6.2.5 is revised as follows.

Replace $u_{n}$ there by $u$, let $B$ be a small neighborhood in $Q_{1} \cap Q_{2} \subset \mathbb{R} \times S^{1}$. Let $P_{0} \in V_{\delta}^{k}\left(J_{0}, X_{0}\right)$ be supported in a small neighborhood $\mathfrak{B} \subset T_{f}$, such that $u^{-1}(\mathfrak{B}) \subset B$, similar to the definition of $\underline{H}_{\lambda_{n}}$ in I.6.2.5. Let $P^{\Lambda}$ be an extension of $P_{0}$ satisfying (104) and (105), which is in turn the analog of $\underline{H}^{\Lambda}$ in I.6.2.5.

Let $\tilde{u}_{1}, \ldots, \tilde{u}_{m}$ be the $m$ distinct lifts of $u$ in $\mathrm{e}^{\nu, m}$. With the above choice of $P_{0}$, the perturbation $\wp_{\chi_{0}} P_{0}\left(\tilde{u}_{i}(s)\right)$ is nontrivial only when $s$ is in the small interval

$$
I_{B}:=\operatorname{pr}_{1}(B)
$$

where $\operatorname{pr}_{1}: \mathbb{R} \times S^{1} \rightarrow \mathbb{R}$ denotes the projection. By construction, the values of $\Omega_{\nu}$ at different lifts of a point in $\mathcal{C}$ differ by multiples of $m$. Thus if $I_{B}$ is sufficiently small, the image $\Omega_{\nu}\left(\tilde{u}_{i}\left(I_{B}\right)\right)$ forms disjoint intervals in $\mathbb{R}$ for different $\tilde{u}_{i}$. We denote the interval corresponding to $\tilde{u}_{i}$ by $\mathcal{J}_{i}$ and choose $\chi_{0}$ such that

$$
\chi_{0}(\phi)=C_{i} \quad \text { when } \phi \in \mathcal{J}_{i}, i=1,2, \ldots, m,
$$

where $C_{i}$ are distinct constants and $\chi_{0}$ is very small in $C_{\epsilon}$-norm. With this choice,

$$
\wp_{\chi_{0} P_{0}}\left(\tilde{u}_{i}(s)\right)=C_{i} \nabla P_{0}\left(\tilde{u}_{i}(s)\right)
$$


and the analog of I.(71) now reads

$$
E_{\tilde{u}_{i}} \xi+\alpha_{i} Y_{\tilde{u}_{i}}+C_{i} \nabla P_{0}\left(\tilde{u}_{i}\right)=0,
$$

this, together with the contraction mapping theorem, shows that $\tilde{u}_{i}$ perturbs into a $\tilde{v}_{i}$, so that $\Pi_{\Lambda} \tilde{v}_{i}-\Pi_{\Lambda} u$ are, up to higher order correction terms, proportional to $C_{i}$. Hence the perturbed flows have distinct values under $\Pi_{\Lambda}$.

As remarked before, the regularity of $\Re$-truncated moduli spaces is unaffected by this perturbation, and thus $\left\{\mathcal{V}^{\nu, m}\left(J_{\lambda}, X_{\lambda} ; \chi_{\lambda}, P_{\lambda}\right)\right\}_{\lambda \in \Lambda}$ satisfies all the $\Re$-truncated versions of I.6.2.3 (1)-(8). In particular, it has all the $\Re$-truncated versions of the properties (RHFS*), except for (RHFS2c), (RHFS3c), and (RHFS4).

To see that the remaining properties also hold, we need to verify that the gluing theorems proven in previous sections still hold. (The arguments for (RHFS4) to appear in Section 7 below depend on the perturbation only through the existence of Fredholm theory, and the linear gluing theorem in Section 1.2.4.)

By construction, $S \cap \Lambda_{\mathrm{db}}=\emptyset$. Thus, no gluing for births or deaths (as in Sections 2-5) is necessary.

The proofs of standard gluing theorems such as Proposition 6.1.1, Lemma 1.2.4 do require updates. However, because of our choice of $P^{\Lambda}, \wp_{\chi_{\lambda} P_{\lambda}}$ vanishes near the critical point $x_{\lambda}$. Thus, we have the usual exponential decay of flows to critical points and the same error estimates. Only two facts need to be verified for the standard arguments sketched in Section 1.2 to go through:

(1) Fredholm theory and surjectivity of the perturbed version of deformation operators $E_{\tilde{v}}^{J_{\lambda}, X_{\lambda} ; \chi_{\lambda}, P_{\lambda}}, \hat{E}_{\tilde{u}}^{J_{\lambda}, X_{\lambda} ; \chi_{\lambda}, P_{\lambda}}$, where $\tilde{u}, \tilde{v}$ are the perturbed flows to be glued;

(2) the usual quadratic bound on the nonlinear term $N_{w_{\chi}}$, namely, (3).

We shall verify these in the next subsection.

6.3. $\Re$-truncated moduli spaces of perturbed flows. The structure theory of the moduli spaces of such perturbed flows is not covered in the literature or in the discussion of Part I. We need to start from scratch and check the foundation of this more general theory. The major components of the expected structure theory are examined in turn below.

We have already mentioned the following basic fact:

6.3.1. Fact (Exponential decay). A perturbed flow decays exponentially to a nondegenerate critical point.

This is due to our choice that $P^{\Lambda} \in V_{\delta}^{\Lambda ; k, \kappa}\left(J^{\Lambda}, X^{\Lambda}\right)$, in particular, $P_{\lambda}$ vanishes up to $k$ th order at the critical points. In fact, this also shows 
that a perturbed flow decays polynomially to a good minimally degenerate critical point as described in Section I.5. However, we do not need this fact.

6.3.2. Fredholm theory. Consider the linearization of $\bar{\partial}_{J X}^{\chi P}\left(u,[\mu]_{m}\right)$. We denote it by $E_{\left(u,[\mu]_{m}\right)}^{J, X ; \chi, P}$ or $D_{\left(u,[\mu]_{m}\right)}^{J, X ; \chi, P}$, depending on whether $\left(u,[\mu]_{m}\right)$ is an connecting flow line or an orbit. In addition to the well-understood $E_{\left(u,[\mu]_{m}\right)}^{J, X}(\xi)$ or $D_{\left(u,[\mu]_{m}\right)}^{J, X}(\xi)$, it has the following extra terms due to $\wp_{\chi P}$ :

$$
\begin{aligned}
& \chi\left(\Omega_{\nu}\left(u,[\mu]_{m}\right)\right) \nabla_{\xi} \nabla P(u) \\
& \quad+\chi^{\prime}\left(\Omega_{\nu}\left(u,[\mu]_{m}\right)\right) \int_{S^{1}} \omega_{\nu}\left(\xi, \partial_{t} u\right) d t \nabla P(u) .
\end{aligned}
$$

Observing that the first term is a 0-th-order multiplicative operator, and the second term is a mixture which is infinitely smoothing in $t$ and 0 -thorder in $s$, this implies that $D_{\left(u,[\mu]_{m}\right)}^{J, X ;, P}$ is still Fredholm. To see that $E_{\left(u,[\mu]_{m}\right)}^{J, X ; \chi, P}$ is Fredholm, we use in addition Fact 6.3.1 above, and the usual excision argument for Fredholmness in this situation.

The deformation operators for parameterized moduli spaces are finiterank stabilizations of the above operators, and their Fredholmness is thus evident from the above discussion.

6.3.3. Estimating the nonlinear term. The contribution of the perturbation to the nonlinear term $N_{\left(u,[\mu]_{m}\right)}^{J, X ; \chi, P}(\xi)$ is

$$
\begin{aligned}
& \chi\left(\Omega_{\nu}\left(u,[\mu]_{m}\right)\right) \nabla_{\xi} \nabla_{\xi} \nabla P(u) \\
& \quad+2 \chi^{\prime}\left(\Omega_{\nu}\left(u,[\mu]_{m}\right)\right) \int_{S^{1}} \omega_{\nu}\left(\xi, \partial_{t} u\right) d t \nabla_{\xi} \nabla P(u) \\
& \quad+\chi^{\prime \prime}\left(\Omega_{\nu}\left(u,[\mu]_{m}\right)\right)\left(\int_{S^{1}} \omega_{\nu}\left(\xi, \partial_{t} u\right) d t\right)^{2} \nabla P(u) \\
& \quad+\chi^{\prime}\left(\Omega_{\nu}\left(u,[\mu]_{m}\right)\right) \int_{S^{1}} \omega_{\nu}\left(\xi, \partial_{t} \xi\right) d t \nabla P(u) .
\end{aligned}
$$

It is straightforward to check that each term above may be bound by

$$
C\|\xi\|_{C^{0}}\|\xi\|_{L_{1}^{p}} \leq C^{\prime}\|\xi\|_{L_{1}^{p}}^{2}
$$

We omit the straightforward estimate for the parameterized version.

6.3.4. Compactness. Let us go over the main ingredients in the usual proof one by one.

- Elliptic regularity. By the above estimate on the nonlinear term, and the form of (106), the elliptic bootstrapping argument still hold, provided a $C^{0}$ bound can be established. The latter relies on the Gromov compactness. 
- Gromov compactness. Going through the rescaling argument, we note that the extra term $\wp_{\chi P}$ disappears in the limit, and therefore again (local) compactness is lost only through bubbling off honest holomorphic spheres. This possibility is eliminated via transversality as in Section 3 of Part I.

- Energy bound. With this definition of nonlocal perturbations, there might not be a good action functional for the perturbed flows. ${ }^{1}$ However, we still have the requisite energy bound for perturbed flows with weight $\leq \Re$. Let $\left(u,[\mu]_{m}\right)$ be such a $\chi P$-perturbed flow. Then

$$
\begin{aligned}
& \mathcal{E}\left(u,[\mu]_{m}\right)=\left\|\partial_{s} u\right\|_{2}^{2} \\
& \quad=\alpha \mathrm{wt}_{-\langle y\rangle, e_{\mathcal{P}}}\left(u,[\mu]_{m}\right)+\int\left\langle\partial_{s} u, \chi\left(\Omega_{\nu}(u,[\mu])\right) \nabla P(u)\right\rangle_{2, t}(s) d s .
\end{aligned}
$$

On the other hand,

Lemma. Let $\left(u,[\mu]_{m}\right)$ be a $\chi$ P-perturbed flow (either a connecting flow line or an orbit). Then there is a constant $C$ independent of $s$ or $\left(u,[\mu]_{m}\right)$, such that

$$
\|\nabla P(u)\|_{2, t}(s) \leq C\left\|\partial_{s} u\right\|_{2, t}(s) \forall s .
$$

Proof. This follows from the $L_{1}^{\infty}$-boundedness of $P$, the fact that $P$ vanishes to up order $k>2$ at the critical points, and the following.

Palais-Smale condition. There exists an $\varepsilon^{\prime}>0$ such that for any $\left(z,[\mu]_{m}\right) \in \mathrm{C}^{\nu, m}$ with $\left\|J(z) \partial_{t} z+\check{\theta}_{X}(z)+\wp_{\chi}\left(z,[\mu]_{m}\right)\right\|_{2, t} \leq \varepsilon^{\prime}$, there is a critical point $z_{0}$ such that $z=\exp \left(z_{0}, \xi\right)$ for a small $\xi$, and

$$
\begin{aligned}
& \left\|J(z) \partial_{t} z+\check{\theta}_{X}(z)+\wp_{\chi} P\left(z,[\mu]_{m}\right)\right\|_{2, t} \geq \\
& \left\{\begin{array}{rr}
C_{1}\|\xi\|_{2, t} \quad \text { when } z_{0} \text { is nondegenerate } \\
C_{2}\|\xi\|_{2, t}^{2} \quad \text { when } z_{0} \text { is minimally degenerate } \\
\quad \text { in a standard d-b neighborhood. }
\end{array}\right.
\end{aligned}
$$

This in turn follows from the Ascoli-Arzela argument as in the unperturbed case, since by our condition on $P, \wp_{\chi} P$ can be ignored near critical points.

Thus, if $\|\chi\|_{C_{\epsilon}} \leq \varepsilon$, the absolute value of the last term in (107) can be bounded by $C \varepsilon\left\|\partial_{s} u\right\|_{2}^{2}$, and by rearranging,

$$
\mathcal{E}\left(u,[\mu]_{m}\right) \leq(1-C \varepsilon)^{-1} \alpha \mathrm{wt}_{-\langle y\rangle, e_{\mathcal{P}}}\left(u,[\mu]_{m}\right) \leq(1-C \varepsilon)^{-1} \alpha \Re .
$$

- Global compactness (for $\hat{\mathcal{M}}_{P}, \hat{\mathcal{M}}_{P}^{\Lambda}$ ). As in the unperturbed case, to go from local compactness to global compactness, we just need in addition Fact 6.3.1.

\footnotetext{
${ }^{1}$ We may easily modify the definition of $\wp_{\chi P}$ so that there is; however we would run into difficulty with Gromov compactness.
} 
6.3.5. Transversality. The transversality arguments in Part I uses a unique continuation theorem extensively. However, Aronszajn's theorem or the Carleman similarity principle used in [7] is no longer applicable as the nonlocal term is introduced. While it might be possible to prove a unique continuation result in this situation, we choose not to develop a general theory here. Instead, for the purpose of proving Proposition 6.1.2, we only need the following.

Claim. Let $(J, X)$ be regular, $\chi$ be sufficiently small (in $C_{\epsilon}$ norm), $P \in$ $V_{\delta}^{k}(J, X)$ and $i \leq 2$. Then for $\mathcal{M}=\mathcal{M}_{P}$ or $\mathcal{M}_{O}, \mathcal{M}_{\nu, m}^{i}\left(J, X ; \chi, P ; \mathrm{wt}_{-\langle\mathcal{P}\rangle, \text { eP }} \leq\right.$ $\Re)$ is (Zariski) smooth. Similarly for the parameterized versions.

Take $\mathcal{M}_{P}$, for example; the arguments for $\mathcal{M}_{O}$ or the parameterized versions are similar. Due to Lemma 1.2.4, for regular $(J, X)$ and $u$ in a neigh-

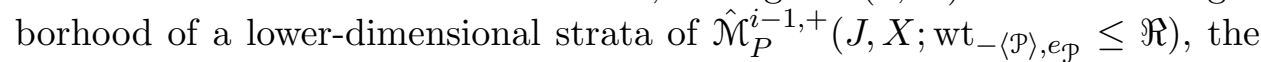
deformation operator $E_{u}$ has a uniformly bounded right inverse. Combining this with the compactness of $\hat{\mathcal{M}}_{P}^{i-1,+}\left(J, X\right.$; wt $\left.-\langle\mathcal{P}\rangle, e_{\mathcal{P}} \leq \Re\right)$, there is a small number $\delta>0$ such that any element in $\left\{\mathfrak{D} \mid \mathfrak{D}-E_{v}^{J X} \|<\delta, v \in\right.$ $\left.\hat{\mathcal{M}}_{P}^{i-1}\left(J, X ; \mathrm{wt}_{-\langle\mathcal{P}\rangle, e_{\mathcal{P}}} \leq \Re\right)\right\}$ is surjective. In particular, there is a $\delta^{\prime}=\delta^{\prime}(\delta)$, such that for any element $w$ in

$$
\left\{\exp (v, \xi) \mid\|\xi\|_{\infty, 1}<\delta^{\prime}, v \in \hat{\mathcal{M}}_{P}^{i-1}\left(J, X ; \mathrm{wt}_{-\langle\mathcal{P}\rangle, e_{\mathcal{P}}} \leq \Re\right)\right\},
$$

$E_{\left(w,\left[\mu_{w}\right]_{m}\right)}^{J X ; \chi P}$ is surjective for any lift $\left(w,\left[\mu_{w}\right]_{m}\right)$ of $w$ in $\mathrm{e}^{\nu, m}, \forall \chi$ with $\|\chi\|_{C_{\epsilon}}<$ $\delta^{\prime}$. Thus, the claim follows from the following.

Lemma. Fix $P, i$, and $\Re$ as above. Then there is an $\varepsilon^{\prime}>0$ such that for all $\chi$ with $\|\chi\|_{C_{\epsilon}}<\varepsilon^{\prime}$, any element $(u,[\mu]) \in \hat{\mathcal{M}}_{P ; \nu, m}^{i-1}\left(J, X ; \chi, P, \mathrm{wt}_{-\langle\mathcal{P}\rangle, e_{\mathcal{P}}} \leq \Re\right)$ is close to $\hat{\mathcal{M}}_{P}^{i-1}\left(J, X\right.$, wt $\left.-\langle\mathcal{P}\rangle, e_{\mathcal{P}} \leq \Re\right)$ in the sense that

$$
(*) \quad u=\exp (v, \xi),\|\xi\|_{\infty, 1}<\delta^{\prime} \quad \text { for some } v \in \hat{\mathcal{M}}_{P}^{i-1}\left(J, X, \mathrm{wt}_{-\langle\mathcal{P}\rangle, e_{\mathcal{P}}} \leq \Re\right) .
$$

Proof. Suppose the contrary. Then there exists a sequence $\left\{\chi_{n}\right\}$ such that $\lim _{n \rightarrow \infty}\left\|\chi_{n}\right\|_{C_{\epsilon}}=0$, and a sequence

$$
\left\{\left(u_{n},\left[\mu_{n}\right]_{m}\right)\right\}_{n} \subset \hat{\mathcal{M}}_{P, m}^{k}\left(J, X ; \chi, P ; \mathrm{wt}_{-\langle\mathcal{P}\rangle, e_{\mathcal{P}}} \leq \Re\right)
$$

such that none of them satisfies $\left(^{*}\right)$. By Gromov compactness theorem, such a sequence $\left\{\left(u_{n},\left[\mu_{n}\right]_{m}\right)\right\}$ must weakly converge to an element $v$ in $\hat{\mathcal{M}}_{P}^{k}\left(J, X ; \mathrm{wt}_{-\langle\mathcal{P}\rangle, e_{\mathcal{P}}} \leq \Re\right)$ together with some bubbles. Since by the regularity of $(J, X)$, there is no such bubble, $\left(u_{n},\left[\mu_{n}\right]_{m}\right)$ are close to $v$, contradicting our assumption.

This also shows that when $\chi$ is sufficiently small and $(J, X)$ regular, these $\chi P$-perturbed flows avoid pseudo-holomorphic spheres, as in the case before perturbation. 


\section{Orientation and signs}

In this section, we tie up the last loose end of this article by addressing all orientation issues so far ignored: we verify (FS4) for the Floer theory described in Section I.3 and show that an admissible $(J, X)$-homotopy satisfies (RHFS4).

In Section 7.2, we show that the various moduli spaces $\mathcal{M}_{P}(x, y), \mathcal{M}_{O}^{1}$ and their parameterized variants are orientable; furthermore, we introduce the notions of coherent orientations for $\mathcal{M}_{P}, \mathcal{M}_{P}^{\Lambda}$ and grading-compatible orientations for $\mathcal{M}_{O}^{1}, \mathcal{M}_{O}^{\Lambda, 2}$ and show that these moduli spaces may be endowed with such orientations. This completes the verification that the formal flow associated to a regular pair $(J, X)$ forms a Floer system. The coherence of orientation is determined by linearized versions of the gluing theorems proven in the previous sections; this is, in fact, why we have postponed this discussion. Compared with the full gluing theory, major simplifications for these linearized versions arise from the fact that we may substitute the complicated polynomially weighted Sobolev spaces used in Sections 2-5 by larger, exponentially weighted versions, due to the removal of constraints from nonlinear aspects of general gluing theory. Furthermore, deformation operators between these exponentially weighted spaces are conjugate to deformation operators between the usual $L_{k}^{p}$ spaces with perturbation by asympotically constant 0 -th-order terms, making it possible to work only with the ordinary Sobolev norms throughout this section.

In Section 7.3, we verify the signs in the expressions for $\mathbb{T}_{P, \mathrm{db}}, \ldots, \mathbb{T}_{O, \mathrm{hs}-\mathrm{s}}$ given in (RHFS4) (cf. Section I.4.3.7). This is obtained by examining the orientations of the K-models used in the proofs of the gluing theorems in previous sections. With this done, the verification that admissible $(J, X)$ homotopies satisfy the assumptions of Proposition I.4.6.3 is complete, which in turn implies the general invariance theorem, Theorem I.4.1.1.

7.1. Basic notions and conventions. We first review some basic materials to fix terminology and conventions.

7.1.1. Orientation for direct sums and exact sequences. Given a direct sum of an ordered $k$-tuple of oriented vector spaces, $E=E_{1} \oplus \cdots \oplus E_{k}$, we orient it by $e_{1} \wedge \cdots \wedge e_{k} \in \operatorname{det} E$, where $e_{i} \in \operatorname{det} E_{i}$ orients $E_{i}$.

An exact sequence of finite-dimensional vector spaces

$$
0 \rightarrow E_{1} \stackrel{i_{1}}{\rightarrow} F_{1} \stackrel{j_{1}}{\rightarrow} E_{2} \stackrel{i_{2}}{\rightarrow} F_{2} \cdots \stackrel{j_{n}-1}{\rightarrow} E_{n} \stackrel{j_{n}}{\rightarrow} F_{n} \rightarrow 0
$$

determines an isomorphism $\bigotimes_{k} \operatorname{det} E_{k} \simeq \bigotimes_{k} \operatorname{det} F_{k}$, by writing

$$
E_{k}=B_{k}^{E} \oplus j_{k-1} B_{k-1}^{F}, \quad F_{k}=i_{k} B_{k}^{E} \oplus B_{k}^{F}
$$

for appropriate oriented subspaces $B_{k}^{E}, B_{k}^{F}$, over which $i_{k}, j_{k}$ restrict to isomorphisms. 
7.1.2. Orientation for determinant lines and K-models. Given a Fredholm operator $\mathfrak{D}: E \rightarrow F$, the determinant line

$$
\operatorname{det} \mathfrak{D}:=\operatorname{det} \operatorname{ker} \mathfrak{D} \otimes \operatorname{det}(\operatorname{coker} \mathfrak{D})^{*} \text {. }
$$

It is well known that for a continuous family (in operator norm) of Fredholm operators, the determinant lines above form a real line bundle over the parameter space, namely the determinant line bundle. We use or $(\mathfrak{D})$ to denote the space of possible orientations for det $\mathfrak{D}$ when it is orientable, and similarly, $\mathfrak{o r}\left(\mathfrak{D}^{\Lambda}\right)$ denotes the space of possible trivializations of the determinant line bundle for the family $\mathfrak{D}^{\Lambda}$ when it is orientable. These are affine spaces under $\mathbb{Z} / 2 \mathbb{Z}$. If $\mathfrak{D}_{\lambda_{1}}, \mathfrak{D}_{\lambda_{2}}$ are elements of the family of operators $\mathfrak{D}^{\Lambda}$, we say that $\mathfrak{o}_{1} \in \mathfrak{o r}\left(\mathfrak{D}_{\lambda_{1}}\right)$ and $\mathfrak{o}_{2} \in \mathfrak{o r}\left(\mathfrak{D}_{\lambda_{2}}\right)$ are correlated via $\mathfrak{D}^{\Lambda}$ if they are restrictions of the same trivialization $\mathfrak{O} \in \mathfrak{o r}\left(\mathfrak{D}^{\Lambda}\right)$. They are said to be of relative sign $\rho \in\{ \pm 1\}$ (with respect to $\mathfrak{D}^{\Lambda}$ ), denoted $\left[\mathfrak{o}_{1} / \mathfrak{o}_{2}\right]$, if $\mathfrak{o}_{1}$ and $\rho \boldsymbol{o}_{2}$ are correlated.

It is convenient to describe the orientation of $\operatorname{det} \mathfrak{D}$ in terms of K-models. Recall the definition of oriented K-models and the exact sequence (6) from Section 1.2.3. This exact sequence induces the isomorphism:

$$
\operatorname{det} \mathfrak{D} \simeq \operatorname{det} K \otimes \operatorname{det} C^{*} .
$$

Thus, an orientation of a K-model for $\mathfrak{D}$ decides an orientation for det $\mathfrak{D}$. Given an orientation of det $\mathfrak{D}$, an oriented K-model $[K ; C]$ of $\mathfrak{D}$ is said to be compatible with this orientation, if the orientation of $[K ; C]$ induces the orientation of $\operatorname{det} \mathfrak{D}$.

Two K-models of $\mathfrak{D}$ are said to be co-oriented if they give rise to the same orientation of det $\mathfrak{D}$. Let $\left[\mathfrak{D}_{\lambda_{1}}: K_{\lambda_{1}} \rightarrow C_{\lambda_{1}}\right]_{B_{\lambda_{1}}},\left[\mathfrak{D}_{\lambda_{2}}: K_{\lambda_{2}} \rightarrow C_{\lambda_{2}}\right]_{B_{\lambda_{2}}}$ be fibers of a family K-model for $\mathfrak{D}^{\Lambda}$. They are said to be mutually co-oriented via the family $\mathfrak{D}^{\Lambda}$ if they are, respectively, compatible with orientations of $\operatorname{det} \mathfrak{D}_{\lambda_{1}}$ and $\operatorname{det} \mathfrak{D}_{\lambda_{2}}$ correlated by $\mathfrak{D}^{\Lambda}$.

7.1.3. Induced orientation of a stabilization. Let $\hat{\mathfrak{D}}_{\Psi}: \mathbb{R}^{k} \oplus E \rightarrow F$ be a stabilization of $\mathfrak{D}: E \rightarrow F$; recall the definition of stabilized K-models from Section 1.2.3.

Given an orientation $\mathfrak{o} \in \mathfrak{o r}(\mathfrak{D})$, we define the induced orientation $\hat{\mathfrak{o}} \in$ $\mathfrak{o r}\left(\hat{\mathfrak{D}}_{\Psi}\right)$ from $\mathfrak{o}$ as follows. Given an oriented K-model $[\mathfrak{D}: K \rightarrow C]_{B}$ compatible with $\mathfrak{o}$, let $\hat{\mathfrak{o}}$ be the orientation given by the stabilization $\left[\hat{\mathfrak{D}}_{\Psi}: \hat{K} \rightarrow C\right]_{\hat{B}}$, where $\hat{K}$ is oriented as

$$
\hat{K}=(-1)^{k \text { ind } \mathfrak{D}} \mathbb{R}^{k} \oplus K .
$$

7.1.4. Reduction of oriented K-models. Let the K-model $\left[K^{\prime} \rightarrow C^{\prime}\right]$ be a reduction of another K-model, $[K \rightarrow C]$, by $Q$ (cf. Section 1.2.3). Then the orientation of one K-model induces an orientation of the other via writing

$$
K=K^{\prime} \oplus Q ; \quad C=C^{\prime} \oplus \Pi_{C} \mathfrak{D}(Q)
$$


as oriented spaces. Note that changing the orientation of $Q$ results in a co-oriented K-model.

7.1.5. Orientation for glued K-models. Recall the definitions and notations in Section 1.2.4. Given an ordered $k$-tuple of finite-dimensional subspaces $K_{1}, \ldots, K_{k}$ in $E$ or $F$, and sufficiently large $R_{1}, \ldots, R_{k}$, we orient the glued space $K_{1} \#_{R_{1}} \cdots \#_{R_{k-1}} K_{k}$ or $K_{1} \#_{R_{1}} \cdots \#_{R_{k-1}} K_{k} \#_{R_{k}}$ by its natural isomorphism with $K_{1} \oplus K_{2} \oplus \cdots \oplus K_{k}$.

Let $\mathfrak{D}_{1}, \mathfrak{D}_{2}$ be an ordered pair of glueable Floer-type operators, and let $\mathfrak{D}$ be a cyclically glueable Floer-type operator. Given $\mathfrak{o}_{1} \in \mathfrak{o r}\left(\mathfrak{D}_{1}\right)$, $\mathfrak{o}_{2} \in \mathfrak{o r}\left(\mathfrak{D}_{2}\right), \mathfrak{o} \in \mathfrak{o r}(\mathfrak{D})$, we define $\mathfrak{o}_{1} \#_{R} \mathfrak{o}_{2} \in \mathfrak{o r}\left(\mathfrak{D}_{1} \#_{R} \mathfrak{D}_{2}\right), \mathfrak{o}_{\#_{R}} \in \mathfrak{o r}\left(\mathfrak{D} \#_{R}\right)$ as follows. Let $\left[K_{1} \rightarrow C_{1}\right],\left[K_{2} \rightarrow C_{2}\right],[K \rightarrow C]$ be oriented $K$-models compatible with $\mathfrak{o}_{1}, \mathfrak{o}_{2}$, and $\mathfrak{o}$, respectively. Then the induced orientation, $\mathfrak{o}_{1} \#{ }_{R} \mathfrak{o}_{2}$ and $\mathfrak{o}_{\# R}$, are, respectively, the orientation given by the oriented K-models

$$
\left[(-1)^{\operatorname{dim}\left(C_{1}\right) \cdot \text { ind } \mathfrak{D}_{2}} K_{1} \#_{R} K_{2} \rightarrow C_{1} \#_{R} C_{2}\right], \quad\left[K_{\# R} \rightarrow C_{\# R}\right] .
$$

The orientations for the generalized kernels and generalized cokernels of stabilized, reduced, or glued K-models given above are chosen such that co-oriented K-models give rise to co-oriented stabilized, reduced, or glued K-models. Thus, we have well-defined homomorphisms of affine spaces under $\mathbb{Z} / 2 \mathbb{Z}$ :

$$
\begin{gathered}
s_{\Psi}: \operatorname{or}(\mathfrak{D}) \rightarrow \mathfrak{o r}\left(\hat{\mathfrak{D}}_{\Psi}\right), \\
\mathfrak{g}_{R}: \operatorname{or}\left(\mathfrak{D}_{1}\right) \times \mathfrak{o r}\left(\mathfrak{D}_{2}\right) \rightarrow \mathfrak{o r}\left(\mathfrak{D}_{1} \#_{R} \mathfrak{D}_{2}\right), \\
\mathfrak{s} \mathfrak{g}_{R}: \operatorname{or}(\mathfrak{D}) \rightarrow \mathfrak{o r}\left(\mathfrak{D}_{\# R}\right),
\end{gathered}
$$

sending $\mathfrak{o}$ to $\hat{\mathfrak{o}}, \mathfrak{o}_{1} \times \mathfrak{o}_{2}$ to $\mathfrak{o}_{1} \#_{R} \mathfrak{o}_{2}$, and $\mathfrak{o}$ to $\mathfrak{o}_{\# R}$, respectively. We call $s_{\Psi}$ the stabilization isomorphism, and $\mathfrak{g}_{R}, \mathfrak{s} \mathfrak{g}_{R}$ the gluing homomorphisms. As a consequence of the independence of K-models, the above constructions also work in the family setting to define induced orientations for the determinant line bundles of stabilized or glued operators. In addition, the gluing homomorphisms above may be extended to be defined for arbitrary $k$-tuple of glueable or cyclically glueable Floer-type operators, by observing that any glued operator or cyclically glued operator can be obtained by a combination of translation and the two gluing operations discussed above. Morever, with the above definition, it is straightforward to check that the oriented K-model for the same glued operator obtained from different combinations are actually the same.

Remarks. (1) Alternatively, one may define induced orientation for stabilization by the oriented K-model $\left[\mathbb{R}^{k} \oplus K \rightarrow C\right]$ instead. We have so chosen our definition because in our context, det $\mathfrak{D}_{\Psi}$ gives the orientation of a fiber bundle, where $\mathbb{R}^{k}$ corresponds to the tangent space of the base. We prefer 
the "fiber-first" convention for orienting a fiber bundle. With our definitions, the gluing homomorphism commutes with the (rank k) stabilization isomorphism on $\mathfrak{D}_{2}$, but commutes with stabilization on $\mathfrak{D}_{1}$ modulo the sign $(-1)^{k \text { ind } \mathfrak{D}_{2}}$.

(2) The definitions of the orientation for a stabilization and glued operators in [4] differ from ours. Their definitions have the following disadvantage. Given an orientation of a determinant line bundle for a family $\left\{\mathfrak{D}_{\lambda}\right\}_{\lambda \in \Lambda}$, the stabilization isomorphism of [4] gives a possibly discontinuous, nowhere vanishing section of the determinant line bundle of the stabilized family $\left\{\hat{\mathfrak{D}}_{\lambda, \Psi}\right\}_{\lambda \in \Lambda}$. Furthermore, the gluing morphisms in [4] commute with stabilization only up to a sign depending on the dimension of $\mathbb{R}^{k}$.

7.2. Orienting moduli spaces. This subsection addresses the orientability issues required by (FS4) and (RHFS4).

By an orientation of a moduli space $\mathcal{M}=\mathcal{M}_{P}$ or $\mathcal{M}_{O}$, we mean the following. Notice that the configuration spaces $\mathcal{B}_{P}^{k}(x, y), \mathcal{B}_{O}^{k}$ parameterize families of deformation operators, $\left\{E_{u} \mid u \in \mathcal{B}_{P}^{k}(x, y)\right\},\left\{\tilde{D}_{(T, u)} \mid(T, u) \in \mathcal{B}_{O}^{k}\right\}$. Thus, they carry determinant line bundles, which we denote by $L \mathcal{B}_{P}^{k}(x, y), L \mathcal{B}_{O}$. The moduli space $\mathcal{M} \subset \mathcal{B}=\mathcal{B}_{P}^{k}(x, y)$ or $\mathcal{B}_{O}^{k}$ parameterizes a subfamily of deformation operators, and thus carries a determinant line bundle $L \mathcal{M}$, which is the pull-back of $L \mathcal{B}$. An orientation of $\mathcal{M}$ will mean a trivialization of $L \mathcal{M}$. In this article, this will always be the pull-back of a trivialization of $L \mathcal{B}$, and we shall therefore focus on orienting $L \mathcal{B}$ for various configuration spaces $\mathcal{B}$. Similarly, parameterized moduli spaces $\mathcal{M}^{\Lambda}$ will be oriented by orienting $L \mathcal{B}^{\Lambda}$. Since the deformation operators for $\mathcal{M}^{\Lambda}$ are stabilizations of those for $\mathcal{M}$, this also orients the fiber moduli spaces $\mathcal{M}_{\lambda}$ for $\lambda \in \Lambda$.

Notice that the above definition does not require nondegeneracy of the moduli spaces $\mathcal{M}$, and hence we make no such assumptions in this subsection. Nevertheless, when $\mathcal{M}$ is nondegenerate, the determinant line for the relevant deformation operator $\operatorname{det} \mathfrak{D}_{u}=\operatorname{det} T_{u} \mathcal{M}$ at any $u \in \mathcal{M}$. In this case, this definition agrees with the usual definition of the orientation of a manifold.

We do, however, assume nondegeneracy of the spaces of critical points. Namely, we assume (FS1) for a Floer theory $\left(\mathcal{C}, \mathfrak{H}\right.$, ind; $\left.y_{\chi}, \mathcal{V}_{\chi}\right)$, and assume (RHFS1*) for a CHFS throughout this subsection.

We now begin with some general discussion on abstract Floer theories in Sections 7.2.1-7.2.4.

7.2.1. General strategy for orientability. Below we roughly outline a scheme to establish orientability of $L \mathcal{B}$, which is particularly useful for symplectic Floer theories, when the configuration spaces have complicated topology. To begin, construct a map

$$
m: \mathcal{B} \rightarrow \Sigma / G,
$$


where $\Sigma$ is a contractible space parameterizing certain operators and $G$ is a suitable automorphism group. The map $m$ is typically defined by identifying the deformation operator at $u \in \mathcal{B}$ to an operator in $\Sigma$, after certain trivialization is chosen. $G$ is usually the group of automorphisms relating different possible trivializations.

The space $\Sigma$ parameterizes a trivial determinant line bundle $L \Sigma$, over which the action by $G$ extends. Moreover, $(L \Sigma) / G=L(\Sigma / G)$ and $L \mathcal{B}=m^{*} L(\Sigma / G)$. One next shows that $G$ induces trivial actions on the determinant lines. Thus $L(\Sigma / G)$, and hence also $L \mathcal{B}$, are trivial.

In family settings, $\mathcal{B}$ and $\Sigma$ above are both replaced by bundles $\mathcal{B}^{\Lambda}, \Sigma^{\Lambda}$ over the parameter space $\Lambda$, and $m$ above will be a bundle map.

In the case $\mathcal{B}=\mathcal{B}_{P}(x, y)$, in order for the deformation operator to be Fredholm, $x, y \in \mathcal{P}$ have to be nondegenerate. More generally, one may consider exponentially weighted versions of deformation operators $E_{u}^{\left(\sigma_{1}, \sigma_{2}\right)}$ (cf. Section I.3.2.3) instead of $E_{u}$. When $x, y$ are, respectively, $\sigma_{1}$-weighted nondegenerate and $\sigma_{2}$-weighted nondegenerate, this defines another determinant line bundle over $\mathcal{B}_{P}(x, y)$, which we denote by $L^{\left(\sigma_{1}, \sigma_{2}\right)} \mathcal{B}_{P}(x, y)$. Under this weighted-nondegeneracy condition on $x, y$, the determinant line bundle $L^{\left(\sigma_{1}, \sigma_{2}\right)} \mathcal{B}_{P}(x, y)$ is independent of small perturbations to the weights $\sigma_{1}, \sigma_{2}$.

These weighted versions are useful for dealing with the case when one of $x, y$ is minimally degenerate: in this case, the deformation operator $E_{u}$ is defined as a map between complicated polynomially weighted Sobolev spaces (cf. Section I.5). However, we showed in Section I.5.2.5 that $E_{u}$ has identical kernel and cokernel as $E_{u}^{(-\sigma, \sigma)}$ for any small positive $\sigma$. As we are only concerned with the linear aspect (Kuranishi structure) of the Floer theory, there is thus no harm in replacing $E_{u}$ by the simpler $E_{u}^{(-\sigma, \sigma)}$ : the orientation of $\mathcal{M}_{P}(x, y)$ in this case will be given by an orientation of $L^{(-\sigma, \sigma)} \mathcal{B}_{P}(x, y)$.

Turning now to the family situation of a CHFS $\left\{\mathcal{V}_{\lambda}\right\}_{\lambda \in \Lambda}$ satisfying properties $\left(\mathrm{RHFS1}^{*}\right)$, given $\mathbf{x}, \mathbf{y} \in \aleph_{\Lambda}$ and an interval $S \subset \Lambda$, let $\mathcal{B}_{P}^{S}(\underline{\mathbf{x}}, \underline{\mathbf{y}})=$ $\bigcup_{\lambda \in S \cap \bar{\Lambda}_{\mathbf{x}} \cap \bar{\Lambda}_{\mathbf{y}}} \mathcal{B}_{P}\left(x_{\lambda}, y_{\lambda}\right)$. Under the assumption $\left(\mathrm{RHFS1}{ }^{*}\right)$, one may choose a set of intervals $\left\{S_{i}\right\}$ covering $\Lambda$, such that each $S_{i}$ contains at most one death-birth, and all overlaps $S_{i} \cap S_{j}$ for different $i, j$ contains no deathbirth. Over each $S_{i}$, one may choose appropriate weights $\sigma_{x, i}, \sigma_{y, i}$ with small absolute value, such that $x_{\lambda}$ is $\sigma_{x, i}$-weighted nondegenerate and $y_{\lambda}$ is $\sigma_{y, i}$-weighted nondegenerate for all $\lambda \in S_{i} \cap \bar{\Lambda}_{\mathbf{x}} \cap \bar{\Lambda}_{\mathbf{y}}$. An orientation of $L^{\left(\sigma_{x, i}, \sigma_{y_{i}}\right)} \mathcal{B}_{P}^{S_{i}}(\underline{\mathbf{x}}, \underline{\mathbf{y}})$ determines an orientation of $\mathcal{M}_{P}^{S_{i}}(\underline{\mathbf{x}}, \underline{\mathbf{y}})$ as well as ones for its fibers $\mathcal{M}_{P, \lambda}(\underline{\mathbf{x}}, \underline{\mathbf{y}})$, which agree with our previous discussion on orienting $\mathcal{M}_{P}(x, y)$ for nondegenerate or minimally degenerate $x, y$. Note again that the precise values of the weights $\sigma_{x, i}, \sigma_{y, i}$ are immaterial; in particular, when $S_{i}$ contains no death-birth, they can be chosen to be 0 . Otherwise, only the signs of these weights matter. 
Lastly, we may patch up the determinant line bundles $L^{\left(\sigma_{x, i}, \sigma_{y_{i}}\right)} \mathcal{B}_{P}^{S_{i}}(\underline{\mathbf{x}}, \underline{\mathbf{y}})$ for all intervals $S_{i}$ to define a determinant line bundle $L \mathcal{B}_{P}^{\Lambda}(\underline{\mathbf{x}}, \underline{\mathbf{y}})$ over $\mathcal{B}_{P}^{\Lambda}(\underline{\mathbf{x}}, \underline{\mathbf{y}})$, by observing that, since for all $\lambda \in \bigcup_{i, j} \overline{S_{i} \cap S_{j}}, x_{\lambda}, y_{\lambda}$ are nondegenerate, determinant line bundles with different weights over $\mathcal{B}_{P}^{\overline{S_{i} \cap S_{j}}}(\underline{\mathbf{x}}, \underline{\mathbf{y}})$ can be identified.

More concretely, in Section 7.2.5 we shall apply the above general scheme to the specific Floer theory described in Section I.3. See also [8] for its application in other versions of symplectic Floer theories. In gauge-theoretic settings, the configuration space $\mathcal{B}$ itself has the structure of $\mathcal{A} / \mathcal{G}$, where $\mathcal{A}$ is an affine space and $\mathcal{G}$ is the gauge group, which is often connected under the assumption of simple-connectivity of the underlying manifold. Thus, much of the above scheme also carries over to this context.

7.2.2. Coherent orientations for $L \mathcal{B}_{P}$. Assuming the orientability of $L \mathcal{B}_{P}^{k}(x, y)$ and $L \mathcal{B}_{P}^{\Lambda, k+1}(\mathbf{x}, \mathbf{y})$ for any pair of $x, y \in \mathcal{P}$ or $\mathbf{x}, \mathbf{y} \in \aleph_{\Lambda}$ and any $k \in \mathbb{Z}$, we explain in Sections 7.2.2-7.2.3 how the orientations of all these should be related, so as to endow the moduli spaces of broken trajectories with a correct oriented manifold-with-corners structure.

Notation. Given a determinant line bundle $L Q$ over a parameter space $Q, L Q \backslash$ zero section contracts to a $\mathbb{Z} / 2 \mathbb{Z}$-bundle over $Q$, which we denote by $\mathfrak{O} \mathfrak{r}(L Q) . \quad L Q$ is orientable if $\mathfrak{O r}(L Q)$ is trivial; in this case, the space of sections of $\mathfrak{O r}(L Q)$ is denoted $\mathfrak{o r}(L Q)$ : this $\mathbb{Z} / 2 \mathbb{Z}$-torsor is the space of possible orientations for $L Q$.

Recall the continuity of the gluing homomorphism $\mathfrak{g}_{R}\left(\mathfrak{D}_{1}, \mathfrak{D}_{2}\right)$ in $\mathfrak{D}_{1}, \mathfrak{D}_{2}$, and $R$. Thus, it defines a gluing homomorphism

$$
\mathfrak{g}: \operatorname{or}\left(L \mathcal{B}_{P}^{k_{1}}\left(z_{1}, z_{2}\right)\right) \times \mathfrak{o r}\left(L \mathcal{B}_{P}^{k_{2}}\left(z_{2}, z_{3}\right)\right) \rightarrow \mathfrak{o r}\left(L \mathcal{B}_{P}^{k_{1}+k_{2}}\left(z_{1}, z_{3}\right)\right)
$$

for any $z_{1}, z_{2}, z_{3} \in \mathcal{P}$ and $k_{1}, k_{2} \in \mathbb{Z}$. We write $\mathfrak{g}\left(o_{1}, o_{2}\right)=o_{1} \# o_{2}$.

Definition. Let $\left(\mathcal{C}, \mathfrak{H}\right.$, ind; $\left.y_{\chi}, \mathcal{V}_{\chi}\right)$ be a Floer theory satisfying (FS1); in particular, $\mathcal{P}$ consists of finitely many nondegenerate elements. A coherent orientation of

$$
L \mathcal{B}_{P}=\coprod_{k \in \mathbb{Z}} \coprod_{x, y \in \mathcal{P}} L \mathcal{B}_{P}^{k}(x, y)
$$

is a section, $\mathfrak{s}$, of $\mathfrak{O} \mathfrak{r}\left(L \mathcal{B}_{P}\right)$, so that for all $k_{1}, k_{2} \in \mathbb{Z}, z_{1}, z_{2}, z_{3} \in \mathcal{P}$,

$$
\left.\left.\mathfrak{s}\right|_{\mathcal{B}_{P}^{k_{1}}\left(z_{1}, z_{2}\right)} \# \mathfrak{s}\right|_{\mathcal{B}_{P}^{k_{2}}\left(z_{2}, z_{3}\right)}=\left.\mathfrak{s}\right|_{\mathcal{B}_{P}^{k_{1}+k_{2}}\left(z_{1}, z_{3}\right)} .
$$

A moment's thought (or cf. [4]) reveals that coherent orientations always exist. Fixing an $x \in \mathcal{P}$, a coherent orientation for $L \mathcal{B}_{P}$ is determined by choosing an element in $\mathfrak{o r}\left(L \mathcal{B}_{P}^{k_{y}}(x, y)\right)$ for each $y \neq x$ and an integer $k=$ $\operatorname{gr}(x, y) \bmod 2 \mathbb{N}_{\psi}$, and in the case when $\mathbb{N}_{\psi} \neq 0$, an additional element of $\mathfrak{o r}\left(L \mathcal{B}_{P}^{2 \mathbb{N}_{\psi}}(x, x)\right)$. The cases of $\operatorname{card}(\mathcal{P})=0$, or $\operatorname{card}(\mathcal{P})=1$ and $\mathbb{N}_{\psi}=0$ are 
excluded: in the first case, $\mathcal{B}_{P}$ is empty, while in the second case, there is no nonconstant connecting flow line. Thus, there is nothing to orient in these cases.

The following fact is immediate from the definition of coherent orientation, but shall be important later.

Lemma. Let $\mathfrak{s}$ be an arbitrary coherent orientation of $L \mathcal{B}_{P}$. Then for any $x \in \mathcal{P},\left.\mathfrak{s}\right|_{\mathcal{B}_{P}^{0}(x, x)}$ is the canonical orientation of $\operatorname{LB}_{P}^{0}(x, x)$.

In the above, the canonical orientation of $\operatorname{LB}_{P}^{0}(x, x)$ is that determined by the canonical orientation of det $E_{\bar{x}}$, the latter being due to the identification of the kernel and cokernel of $E_{\bar{x}}=d / d s+A_{x}$ via the facts that ker $E_{\bar{x}}=$ ker $A_{x}$, coker $E_{\bar{x}}=$ coker $A_{x}$, and that $A_{x}$ is self-adjoint.

Proof. The coherence condition (109) requires the gluing maps

$$
\begin{aligned}
\mathfrak{g}\left(\left.\mathfrak{s}\right|_{\mathcal{B}_{P}^{0}(x, x)},-\right): & \mathfrak{o r}\left(L \mathcal{B}_{P}^{k}(x, y)\right) \rightarrow \operatorname{or}\left(L \mathcal{B}_{P}^{k}(x, y)\right) ; \\
\mathfrak{g}\left(-,\left.\mathfrak{s}\right|_{\mathcal{B}_{P}^{0}(x, x)}\right): & \mathfrak{o r}\left(L \mathcal{B}_{P}^{k^{\prime}}(z, x)\right) \rightarrow \mathfrak{o r}\left(L \mathcal{B}_{P}^{k^{\prime}}(z, x)\right)
\end{aligned}
$$

to be the identity map.

\subsubsection{Coherent orientations for $L \mathcal{B}_{P}^{\Lambda}$. Given a CHFS}

$\left\{\left(\mathcal{C}, \mathfrak{H} \text {, ind; } y_{\lambda}, \mathcal{V}_{\lambda}\right)\right\}_{\lambda \in \Lambda}$ satisfying $\left(\mathrm{RHFS}^{*}\right)$, we aim to orient $L \mathcal{B}_{P}^{\Lambda}$, where

$$
\mathcal{B}_{P}^{\Lambda}=\coprod_{k \in \mathbb{Z}} \coprod_{\mathbf{x}, \mathbf{y} \in \aleph_{\Lambda}} \mathcal{B}_{P}^{\Lambda, k}(\underline{\mathbf{x}}, \underline{\mathbf{y}}) \text {. }
$$

There is a natural projection map $\Pi_{\Lambda}: \mathcal{B}_{P}^{\Lambda} \rightarrow \Lambda$, whose fiber over $\lambda \in \Lambda$ is:

- $\mathcal{B}_{P, \lambda}=\coprod_{x_{\lambda}, y_{\lambda} \in \mathcal{P}_{\lambda}} \mathcal{B}_{P}\left(x_{\lambda}, y_{\lambda}\right)$, when $\lambda$ is not a death-birth,

- $\mathcal{B}_{P, \lambda}=\coprod_{x_{\lambda}, y_{\lambda} \in \mathcal{P}_{\lambda} \backslash\left\{z_{\lambda}\right\} \cup\left\{z_{\lambda+}, z_{\lambda-}\right\}} \mathcal{B}_{P}\left(x_{\lambda}, y_{\lambda}\right)$, when $\mathcal{P}_{\lambda}$ contains a unique minimally degenerate critical point $z_{\lambda}$.

The elements $z_{\lambda+}, z_{\lambda-}$ should be regarded as the end points of the two path components $\mathbf{z}_{+}, \mathbf{z}_{-}$of $\mathcal{P}^{\Lambda} \backslash \mathcal{P}^{\Lambda, \text { deg }}$ connected at $z_{\lambda}$, with $\operatorname{ind}\left(\mathbf{z}_{+}\right)=\operatorname{ind}_{+}(z)$, $\operatorname{ind}\left(\mathbf{z}_{-}\right)=$ind $_{-}(z)$ respectively. We write

$$
L \mathcal{B}_{P}\left(x_{\lambda}, z_{\lambda \pm}\right)=\rho_{\lambda}^{*} L \mathcal{B}_{P}^{\Lambda}\left(\underline{\mathbf{x}}, \underline{\mathbf{z}}_{ \pm}\right)=L^{\left(\sigma_{x}, \mp \sigma\right)} \mathcal{B}_{P}\left(x_{\lambda}, z_{\lambda}\right), \quad \text { for } 0<\sigma \ll 1,
$$

where $\rho_{\lambda}: \mathcal{B}_{P, \lambda} \hookrightarrow \mathcal{B}_{P}^{\Lambda}$ is the inclusion.

First, observe that it is also useful to identify $\operatorname{det} E_{u}^{\left(\sigma_{1}, \sigma_{2}\right)}$ with $\operatorname{det} E_{u}^{\left[\sigma_{1}, \sigma_{2}\right]}$, where

$$
E_{u}^{\left[\sigma_{1}, \sigma_{2}\right]}:=\varsigma^{\sigma_{1}, \sigma_{2}} E_{u}\left(\varsigma^{\sigma_{1}, \sigma_{2}}\right)^{-1}=E_{u}+\left(\sigma_{2} s \beta(s)+\sigma_{1} s \beta(-s)\right)^{\prime}
$$


is a map between ordinary Sobolev spaces. With this identification, one may extend the gluing homomorphism to the weighted case:

$$
\mathfrak{g}: \operatorname{or}\left(L^{\left(\sigma_{1}, \sigma_{2}\right)} \mathcal{B}_{P}^{k_{1}}\left(z_{1}, z_{2}\right)\right) \times \mathfrak{o r}\left(L^{\left(\sigma_{2}, \sigma_{3}\right)} \mathcal{B}_{P}^{k_{2}}\left(z_{2}, z_{3}\right)\right) \rightarrow \operatorname{or}\left(L^{\left(\sigma_{1}, \sigma_{3}\right)} \mathcal{B}_{P}^{k_{1}+k_{2}}\left(z_{1}, z_{3}\right)\right) \text {. }
$$

For any triple $\mathbf{z}_{1}, \mathbf{z}_{2}, \mathbf{z}_{3} \in \aleph_{\Lambda}$ with $\bar{\Lambda}_{\mathbf{z}_{1}} \cap \bar{\Lambda}_{\mathbf{z}_{2}} \cap \bar{\Lambda}_{\mathbf{z}_{3}} \neq \emptyset$, and any pair of integers $k_{1}, k_{2}$, one has also the parameterized version of gluing homomorphism

$$
\mathfrak{g}^{\Lambda}: \operatorname{or}\left(L \mathcal{B}_{P}^{\Lambda, k_{1}}\left(\underline{\mathbf{z}}_{1}, \underline{\mathbf{z}}_{2}\right)\right) \times \mathfrak{o r}\left(L \mathcal{B}_{P}^{\Lambda, k_{2}}\left(\underline{\mathbf{z}}_{2}, \underline{\mathbf{z}}_{3}\right)\right) \rightarrow \mathfrak{o r}\left(L \mathcal{B}_{P}^{\Lambda, k_{1}+k_{2}}\left(\underline{\mathbf{z}}_{1}, \underline{\mathbf{z}}_{3}\right)\right)
$$

extending the gluing homomorphism $\mathfrak{g}$ over the fibers $L \mathcal{B}_{P, \lambda}$.

Definition. Given a CHFS satisfying (RHFS1*) as above, a coherent orientation of $L \mathcal{B}_{P}^{\Lambda}$ is a section, $\mathfrak{s}$, of $\mathfrak{O} \mathfrak{r}\left(L \mathcal{B}_{P}^{\Lambda}\right)$, so that:

(1) For any triple $\mathbf{z}_{1}, \mathbf{z}_{2}, \mathbf{z}_{3} \in \aleph_{\Lambda}$ with $\bar{\Lambda}_{\mathbf{z}_{1}} \cap \bar{\Lambda}_{\mathbf{z}_{2}} \cap \bar{\Lambda}_{\mathbf{z}_{3}} \neq \emptyset$, and any pair of integers $k_{1}, k_{2}$,

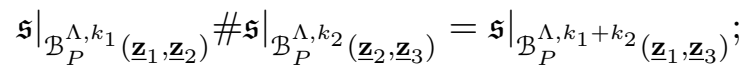

(2) For all $y_{\lambda} \in \mathcal{P}^{\Lambda, \mathrm{deg}},\left.\mathfrak{s}\right|_{\left.\mathcal{B}_{P}^{1}(y+, y-)\right)}$ is the standard orientation of $L^{(-\sigma, \sigma)} \mathcal{B}_{P}^{1}\left(y_{\lambda}, y_{\lambda}\right)$, namely, the orientation given by the oriented K-model $\left[E_{\bar{y}_{\lambda}}^{(-\sigma, \sigma)}: \mathbb{R} \mathbf{e}_{y_{\lambda}} \rightarrow *\right]_{B}$.

Again, it is easy to see that such coherent orientation always exists. When $\mathbb{N}_{\psi} \neq 0$, there are card $\left(\aleph_{\Lambda}\right)$ possible coherent orientations. When $\mathbb{N}_{\psi}=0$, there are $\operatorname{card}\left(\aleph_{\Lambda}\right)-1$ of them. Condition 2 in the above definition is imposed so that the short flow line between the two new critical points $y_{\lambda \pm}$ described in Section 5.3 has positive sign.

7.2.4. Grading-compatible orientation for $L \mathcal{B}_{O}^{1}$. The definition of our invariant $I_{F}$ involves both $\mathcal{M}_{O}^{1}$ and $\mathcal{M}_{P}^{1}$, which are related by gluing elements in $\mathcal{M}_{P}^{0}(x, x)$ during a CHFS. It is thus crucial to orient $\mathcal{M}_{P}^{0}(x, x)$ and $\mathcal{M}_{O}^{1}$ consistently. The notion of "grading compatible orientation" describes such a suitable compatibility relation. More generally, one may consider compatibility conditions relating the orientations of higher-dimensional moduli spaces $\mathcal{M}_{O}^{2 k \mathbb{N}_{\psi}+1}$, and $\mathcal{M}_{P}(x, x)^{2 k \mathbb{N}_{\psi}}$, but this does not concern us, since our invariant involves only low-dimensional moduli spaces.

Let $\mathcal{B}_{O}^{k} \subset \mathcal{B}_{O}$ be the subset consisting of elements $(T, u)$ with $\operatorname{gr}(u)=$ $k-1$, and $L \mathcal{B}_{O}^{k}$ be the determinant line bundle of the family of deformation operators $\tilde{D}_{(T, u)}$. Assume that $L \mathcal{B}_{O}^{1}$ is orientable.

Recall that the relative grading gr in a Floer theory is typically defined via spectral flow by identifying deformation operators $A_{x}$ with elements in a space of self-adjoint operators $\Sigma_{C}$ (cf. Section I.3.1.4 for the version relevant to this article). On the other hand, the orientation of $L \mathcal{B}_{O}^{1}$ is defined by a map $m_{O}: \mathcal{B}_{O}^{1} \rightarrow \Sigma_{O}^{1} / G_{O}$, where $\Sigma_{O}^{1}$ is a space of Fredholm operators of index 1, which includes rank-1 stablizations of the operator

$$
D_{\mathbb{A} ; T}:=\partial_{s}+\mathbb{A}, \quad s \in S_{T}^{1}
$$

for any surjective $\mathbb{A} \in \Sigma_{C}$ and $T \in \mathbb{R}^{+}$. 
Definition. For a Floer theory $\left(\mathcal{C}, \mathfrak{H}\right.$, ind; $\left.y_{\chi}, \mathcal{V}_{\chi}\right)$, the gradingcompatible orientation of $L \mathcal{B}_{O}^{1}$, or more generally $L \Sigma_{O}^{1} / G_{O}$ (also called the orientation compatible with the absolute $\mathbb{Z} / 2 \mathbb{Z}$-grading ind), is the orientation given by the canonical orientation of det $\tilde{D}_{\mathbb{A}, T}$, where $\tilde{D}_{\mathbb{A}, T} \in \Sigma_{O}$ is the rank-1 stabilization of $D_{\mathbb{A}, T}$ by the zero map, and $\mathbb{A}$ is a surjective operator in $\Sigma_{C}$ of even index.

In the above, the canonical orientation of $\tilde{D}_{\mathbb{A}, T}$ is the stabilization of the canonical orientation of $D_{\mathbb{A}, T}$, which in turn is defined in the same way as the canonical orientation of $E_{\bar{x}}$ (cf. Section 7.2.2). Note that the choice of $\mathbb{A}$ and $T$ do not matter in the above definition: as on varies $T, D_{\mathbb{A}, T}$ remains surjective; on the other hand, the independence of the choice of $\mathbb{A}$ is a consequence of the following basic Lemma.

Lemma. For any two surjective operators $\mathbb{A}, \mathbb{A}^{\prime} \in \Sigma_{C}$, the canonical orientations of $\operatorname{det} D_{\mathbb{A}, T}$ and $\operatorname{det} D_{\mathbb{A}^{\prime}, T}$ are of relative sign $(-1)^{\operatorname{gr}\left(\mathbb{A}, \mathbb{A}^{\prime}\right)}$ with respective to the family $\left\{D_{\mathbb{A}, T} \mid \mathbb{A} \in \Sigma_{C}\right\}$, where $\operatorname{gr}\left(\mathbb{A}, \mathbb{A}^{\prime}\right)$ denotes the relative index between $\mathbb{A}$ and $\mathbb{A}^{\prime}$.

An immediate corollary is as follows.

Corollary. Suppose that $L \Sigma_{O}^{1} / G_{O}$ is orientable. Then for any surjective $\mathbb{A} \in \Sigma_{C}$ and $T \in \mathbb{R}^{+}$, the relative sign between the grading-compatible orientation of $L \Sigma_{O}^{1} / G_{O}$ and the canonical orientation of $\operatorname{det} D_{\mathbb{A}, T}$ is $(-1)^{\text {ind } A}$.

7.2.5. Orientability in symplectic Floer theory. We now apply the general strategy described in Section 7.2.1 to establish the orientability of moduli spaces for the specific version of Floer theory considered in this article.

(1) Orienting $L \mathcal{B}_{P}$. This follows from [4], which we now review in our terminology. Let $J \in \mathcal{J}_{K}^{\text {reg }}, X$ be $J$-nondegnerate (cf. Section I.3.2.1), and $\Sigma_{C}$ be as in Section I.3.1.4. Given two self-adjoint, surjective operators $A_{-}, A_{+} \in \Sigma_{C}$, let $\Sigma_{P}\left(A_{-}, A_{+}\right)$be the space of operators of the form: ${ }^{2}$

$$
\partial_{s}+J(s, t) \partial_{t}+\nu(s, t): L_{1}^{p}\left(\mathbb{R} \times S^{1} ; \mathbb{R}^{2 n}\right) \rightarrow L^{p}\left(\mathbb{R} \times S^{1} ; \mathbb{R}^{2 n}\right),
$$

where $J$ is a smooth complex structure on the trivial $\mathbb{R}^{2 n}$-bundle over $\mathbb{R} \times S^{1}$, compatible with the standard symplecic structure on $\mathbb{R}^{2 n} . \quad \nu$ is a smooth matrix-valued function, and both $J$ and $\nu$ extend smoothly over the cylinder $[-\infty,+\infty] \times S^{1}$ that compactifies $\mathbb{R} \times S^{1}$. Furthermore, over the two circles at infinity,

$$
J(-\infty, t) \partial_{t}+\nu(-\infty, t)=A_{-} ; \quad J(\infty, t) \partial_{t}+\nu(\infty, t)=A_{+} .
$$

\footnotetext{
${ }^{2} \Sigma_{P}\left(A_{-}, A_{+}\right)$is basically the space $\Theta$ in Proposition 7 of [4].
} 
The contractibility of $\Sigma_{P}\left(A_{-}, A_{+}\right)$follows from well-known contractibility of the space of complex structures, and the fact that $\nu$ lies in a vector space.

Next, denote by $\mathfrak{T}_{x}$ the space of unitary trivializations of $x^{*} K$ for $x \in$ $\mathcal{P}$ and let $\mathfrak{T}=\coprod_{x \in \mathcal{P}} \mathfrak{T}_{x}$. This is a $C^{\infty}\left(S^{1}, U(n)\right)$-bundle over $\mathcal{P}$. Fix a $g \in C^{\infty}\left(S^{1}, U(n)\right)$ and a section $\Phi: \mathcal{P} \rightarrow \mathfrak{T}$, such that the inclusion $\mathfrak{S}:=$ $\left\{g^{k} \Phi(x) \mid k \in \mathbb{Z}, x \in \mathcal{P}\right\} \hookrightarrow \mathfrak{T}$ induces an isomorphism $i_{\pi}: \mathfrak{S}=\pi_{0} \mathfrak{S} \rightarrow \pi_{0} \mathfrak{T}$.

Recall from Section I.3.1.4 that we have a bundle map (over $\mathcal{P}$ ) from $\tilde{\mathcal{P}}$ to $\pi_{0} \mathfrak{T}$ : from a fixed unitary trivialization of $\gamma_{0}^{*} K$ and a path $w \subset \mathcal{C}$ from $\gamma_{0}$ to $x \in \mathcal{P}$, we extend the trivialization over $w^{*} K$ to obtain a homotopy class of trivializations of $x^{*} K$. If $w^{\prime}$ is another path in the same equivalence class, i.e., $\operatorname{im}\left[w-w^{\prime}\right]=0$, then $\left(w-w^{\prime}\right)^{*} K$ is trivial, since $c_{1}^{f}\left(\operatorname{im}\left[w-w^{\prime}\right]\right)=$ 0 . Hence $w, w^{\prime}$ induce the same homotopy class of trivializations of $x^{*} K$. Composing this map with $i_{\pi}^{-1}$, we have a map assigning to each $(x,[w]) \in \tilde{\mathcal{P}}$ a trivialization $\Phi_{x,[w]} \in \mathfrak{S}_{x}$. Let

$$
\mathbb{A}_{(x,[w])}:=\Phi_{x,[w]} A_{x} \Phi_{x,[w]}^{-1} .
$$

We have a map $m_{P}: \mathcal{B}_{P}^{k}((x,[w]),(y,[v])) \rightarrow \Sigma_{P}\left(\mathbb{A}_{(x,[w])}, \mathbb{A}_{(y,[v])}\right) / G_{P}$, defined as follows.

A $u \in \mathcal{B}_{P}((x,[w]),(y,[v]))$, together with a trivialization $\Phi_{u}$ of the symplectic vector bundle $u^{*} K$ that restricts, respectively, to $\Phi_{(x,[w])}$ and $\Phi_{(y,[v])}$ at the circles at $-\infty$ and $\infty$, assigns an element in $\Sigma_{P}$. Namely, $\mathbb{E}_{u}:=\Phi_{u *} E_{u} \Phi_{u *}^{-1}$.

The space of such trivializations $\Phi_{u}$ is an affine space under

$$
G_{P}=\left\{\Psi\left|\Psi \in C^{\infty}\left([-\infty, \infty] \times S^{1}, \operatorname{Sp}_{n}\right), \Psi\right|_{\{ \pm \infty\} \times S^{1}}=1\right\} .
$$

Let $m_{P}(u)$ be the $G_{P}$-orbit of $\mathbb{E}_{u}$. It is shown in Lemma 13 of [4] that any orbit of $G_{P}$ in $\mathfrak{O r}\left(L \Sigma_{P}\left(\mathbb{A}_{(x,[w])}, \mathbb{A}_{(y,[v])}\right)\right)$ is contained in a single-path component of $\mathfrak{D r}\left(L \Sigma_{P}\left(\mathbb{A}_{(x,[w])}, \mathbb{A}_{(y,[v])}\right)\right)$; thus, $L\left(\Sigma_{P}\left(\mathbb{A}_{(x,[w])}, \mathbb{A}_{(y,[v])}\right) / G_{P}\right)$ is trivial; hence so is $L \mathcal{B}_{P}((x,[w]),(y,[v]))$.

Notice that by definition, for any $\left(z_{1},\left[v_{1}\right]\right),\left(z_{2},\left[v_{2}\right]\right),\left(z_{2},\left[v_{3}\right]\right) \in \tilde{\mathcal{P}}$, $\left.\left.\left(\mathbb{E}_{1}, \mathbb{E}_{2}\right) \in \Sigma_{P}\left(\mathbb{A}_{\left(z_{1},\left[v_{1}\right]\right)}, \mathbb{A}_{\left(z_{2},\left[v_{2}\right]\right)}\right)\right) \times \Sigma_{P}\left(\mathbb{A}_{\left(z_{2},\left[v_{2}\right]\right)}, \mathbb{A}_{\left(z_{3},\left[v_{3}\right]\right)}\right)\right)$ is glueable. This gives rise to a gluing homomorphism

$$
\begin{gathered}
\operatorname{or}\left(L \mathcal{B}_{P}\left(\left(z_{1},\left[v_{1}\right]\right),\left(z_{2},\left[v_{2}\right]\right)\right) \times \operatorname{or}\left(L \mathcal{B}_{P}\left(\left(z_{2},\left[v_{2}\right]\right),\left(z_{3},\left[v_{3}\right]\right)\right)\right.\right. \\
\rightarrow \operatorname{or}\left(L \mathcal{B}_{P}\left(\left(z_{1},\left[v_{1}\right]\right),\left(z_{3},\left[v_{3}\right]\right)\right) .\right.
\end{gathered}
$$

Lastly, observe that if $\operatorname{gr}\left((x,[w),(y,[v]))=\operatorname{gr}\left(\left(x,\left[w^{\prime}\right],\left(y,\left[v^{\prime}\right]\right)\right)=k\right.\right.$, the spaces $\left.\Sigma_{P}\left(\mathbb{A}_{(x,[w])}, \mathbb{A}_{(y,[v])}\right)\right)$ and $\left.\Sigma_{P}\left(\mathbb{A}_{\left(x,\left[w^{\prime}\right]\right)}, \mathbb{A}_{\left(y,\left[v^{\prime}\right]\right)}\right)\right)$ may be identified via conjugation by $\bar{g}^{i}$ for some $i \in \mathbb{Z}$ and $\bar{g} \in C^{\infty}\left([-\infty, \infty] \times S^{1}, \operatorname{Sp}_{n}\right), \bar{g}(s, t):=$ $g(t)$. Thus, the above discussion in fact verifies the orientability of $L \mathcal{B}_{P}^{k}(x, y)$ for any $x, y \in \mathcal{P}, k \in \mathbb{Z}$, and the gluing homomorphism above gives the gluing homomorphism $\mathfrak{g}$ described in Section 7.2.3. 
(2) Orienting $L \mathcal{B}_{P}^{\Lambda}$. Suppose $\left(J^{\Lambda}, X^{\Lambda}\right)$ generates a CHFS satisfying the properties $\left(\mathrm{RHFS}^{*}\right)$. Let $(\mathbf{x},[\mathbf{w}]),(\mathbf{y},[\mathbf{v}])$ be two path components of the space $\tilde{\mathcal{P}}^{\Lambda} / \tilde{\mathcal{P}}^{\Lambda}$,deg.

The deformation operator of $\mathcal{M}_{P}^{\Lambda}$ at $u_{\lambda}, \hat{E}_{u_{\lambda}}$, may be regarded as a stabilization of $E_{u_{\lambda}}$. Because of the stabilization isomorphism for families, to orient the determinant line bundle $\operatorname{det}\left\{\hat{E}_{u_{\lambda}}\right\}_{u_{\lambda} \in \mathcal{B}_{P}^{\Lambda}((\mathbf{x},[\mathbf{w}]),(\mathbf{y},[\mathbf{v}]))}$, it is equivalent to orient the determinant line bundle

$$
L \mathcal{B}_{P}^{\Lambda}((\mathbf{x},[\mathbf{w}]),(\mathbf{y},[\mathbf{v}])):=\operatorname{det}\left\{E_{u_{\lambda}}\right\}_{u_{\lambda} \in \mathcal{B}_{P}^{\Lambda}((\mathbf{x},[\mathbf{w}]),(\mathbf{y},[\mathbf{v}]))} .
$$

This can be oriented by repeating part (1) above, replacing $\Sigma_{P}$ by the parameterized version:

$$
\Sigma_{P}\left(\mathbf{A}_{-}, \mathbf{A}_{+}\right):=\bigcup_{\lambda \in \Lambda_{\mathbf{x}} \cap \Lambda_{\mathbf{y}}} \Sigma_{P}\left(\mathbb{A}_{\left(x_{\lambda},\left[w_{\lambda}\right]\right)}, \mathbb{A}_{\left(y_{\lambda},\left[v_{\lambda}\right]\right)}\right),
$$

which is a $\Sigma_{P}$-bundle over $\Lambda_{\mathbf{x}} \cap \Lambda_{\mathbf{y}}$. In the above, $\mathbb{A}_{\left(x_{\lambda},\left[w_{\lambda}\right]\right)} \forall\left(x_{\lambda},\left[w_{\lambda}\right]\right) \in \tilde{\mathcal{P}}$ is defined via a smooth section $\Phi^{\Lambda}: \mathcal{P}^{\Lambda} \rightarrow \mathfrak{T}^{\Lambda}, \mathfrak{T}^{\Lambda}:=\bigcup_{x_{\lambda} \in \mathcal{P}^{\Lambda}} \mathfrak{T}_{x_{\lambda}}$. This is again a contractible space, since it is a bundle with contractible fibers and base.

As in (1), this in turn demonstrates the orientability of $L \mathcal{B}^{\Lambda, k+1}(\mathbf{x}, \mathbf{y})$ and defines the parameterized version of gluing homomorphism $\mathfrak{g}^{\Lambda}$ described in Section 7.2.3. Now one may follow the arguments in Section 7.2.3 to define a coherent orientation of $L \mathcal{B}_{P}^{\Lambda}$.

(3) Orienting $L \mathcal{B}_{O}^{1}$ and $L \mathcal{B}_{O}^{\Lambda, 2}$. Since $\tilde{D}_{(T, u)}$ is a rank-1 stabilization of $D_{(T, u)}$, it is equivalent to orient $\underline{L} \mathcal{B}_{O}^{1}:=\operatorname{det}\left\{D_{(T, u)}\right\}_{(T, u) \in \mathcal{B}_{O}^{1}}$.

Similarly to parts (1), (2) above, we introduce a map $m_{O}: \mathcal{B}_{O}^{1} \rightarrow \Sigma_{O}^{1} / G_{O}$, where $\Sigma_{O}^{1}$ is the space of rank-1 stabilizations of operators of the form:

$$
\bar{\partial}_{J, \nu ; T}:=\partial_{s}+J(s, t) \partial_{t}+\nu(s, t): L_{1}^{p}\left(S_{T}^{1} \times S^{1} ; \mathbb{R}^{2 n}\right) \rightarrow L^{p}\left(S_{T}^{1} \times S^{1} ; \mathbb{R}^{2 n}\right)
$$

for some $T \in \mathbb{R}^{+}$, with $J, \nu$ defined similarly to part (1). The determinant line bundle $L \Sigma_{O}^{1}$ is canonically oriented as follows. Note that $\Sigma_{O}^{1}$ contracts to the subspace consisting of complex linear $\bar{\partial}_{J, \nu ; T}$, which we denote by $\Sigma_{O}^{\prime}$. However, $L \Sigma_{O}^{\prime}$ is canonically oriented by the complex linearity of kernels and cokernels. Next, note that $u^{*} K$ is trivial for any $(T, u) \in \mathcal{B}_{O}^{1}$. Given a $(u, T) \in \mathcal{B}_{O}^{1}$ and a trivialization $\Phi_{u}$ of $u^{*} K$, one has

$$
\tilde{\mathbb{D}}_{(T, u)}:=\Phi_{u *} \tilde{D}_{(T, u)} \tilde{\Phi}_{u *}^{-1} \in \Sigma_{O},
$$

where $\tilde{\Phi}_{u *}^{-1}:=1 \oplus \Phi_{u *}^{-1}$ as an endomorphism of $\mathbb{R} \oplus L_{1}^{p}\left(S_{T}^{1} \times S^{1} ; \mathbb{R}^{2 n}\right)$. This defines $m_{O}$.

It is not hard to see that $G_{O}$ acts trivially on the $\mathbb{Z} / 2 \mathbb{Z}$-bundle $\mathfrak{O} \mathfrak{r}\left(L \Sigma_{O}^{1}\right)$ by conjugation: by continuation (cf. the commutative diagram in p. 28 of [4]), it suffices to check this for $\mathfrak{D} \mathfrak{r}\left(L \Sigma_{O}^{\prime}\right)$. However, for $\mathfrak{O} \mathfrak{r}\left(L \Sigma_{O}^{\prime}\right)$, this is obvious, again by the complex linearity of elements in $\Sigma_{O}^{\prime}$. 
The orientability of $L \mathcal{B}_{O}^{\Lambda, 2}$ follows immediately from that of $L \mathcal{B}_{O}^{1}$, since $\mathcal{B}_{O}^{\Lambda, 2}=\mathcal{B}_{O}^{1} \times \Lambda$ by definition.

Finally, note that for this version of symplectic Floer theory, the canonical orientation of $L \Sigma_{O}^{1} / G_{O}$ is compatible with the mod 2 Conley-Zehnder index ind: the former is given by the canonical orientation of det $\tilde{D}_{\mathbb{A}_{0}, T}$, where $\mathbb{A}_{0}$ is such that $D_{\mathbb{A}_{0}, T}$ is complex linear. By the definition of Conley-Zehnder index (cf. Section I.3.1.4), $\mathrm{CZ}\left(\mathbb{A}_{0}\right)$ is even.

7.3. The signs. It was shown in $[4]$ that with a coherent orientation for $\mathcal{M}_{P}$, the Floer complex indeed satisfies $\tilde{\partial}_{F}^{2}=0$. In this subsection, we generalize this result to the setting of CHFSs and verify the second statement of (RHFS4). Namely, we show that with $\mathcal{M}_{P}^{\Lambda}$ endowed with coherent orientations and $\mathcal{M}_{O}^{\Lambda, 2}$ endowed with the grading-compatible orientation, the various 0 -dimensional strata $\mathbb{J}_{P}, \mathbb{T}_{P, \text { hs-s }}, \mathbb{T}_{P, \mathrm{db}}$ in $\hat{\mathcal{M}}_{P}^{\Lambda, 1,+}$ and their analogs for broken orbits are expressed in terms of products of 0 -dimensional moduli spaces, with relative signs given by the formulae (I.28-I.33).

As the signs for $\mathbb{J}_{P}, \mathbb{J}_{O}$ given in $(\mathrm{I} .28,31)$ follow immediately from the definition of coherent orientation, we shall concentrate on the signs for $\mathbb{T}_{P, \text { hs-s }}, \mathbb{T}_{O, \text { hs-s }}, \mathbb{T}_{P, \mathrm{db}}$, and $\mathbb{T}_{O, \mathrm{db}}$ : the formulae (I.30, 33, 29, 32) are, respectively, rephrased in terms of the gluing theorems Propositions 2.1, 6.1.1 in Lemmas 7.3.2-7.3.5 below.

We assume throughout this subsection that $L \Sigma_{P} / G_{P}, L \Sigma_{O} / G_{O}$ and their parameterized versions are endowed with coherent orientations/gradingcompatible orientations, and all the oriented K-models are compatible with these orientations, unless otherwise specified.

The results and arguments in this subsection apply to general Floer theory, in which the relevant moduli spaces are oriented according to the scheme in Sections 7.2.1-7.2.4 above.

7.3.1. Preparations. (1) Signs of flowlines. The sign of a flow in a 0 -dimensional reduced moduli space, $\hat{u} \in \mathcal{M}^{1} / \mathbb{R}$, in general, means the relative sign $\left[u^{\prime}\right] /$ ker $\mathfrak{D}_{u}$ for any representative $u \in \mathcal{M}^{1}$ in the unreduced moduli space, where $\mathfrak{D}_{u}$ is the deformation operator of $\mathcal{M}^{1}$. It will be denotedy by $\operatorname{sign}(u)$.

(2) Trivializations of deformation operators. Instead of working with the deformation operators $E_{u}, \tilde{D}_{(T, u)}$ and their parameterized versions, it is often more convenient to work with their corresponding operators in $\Sigma_{P}$ or $\Sigma_{O}$ via lifts of the maps $m_{P}, m_{O}$. These will be denoted by boldface letters such as $\mathbb{E}_{u}, \tilde{\mathbb{D}}_{(T, u)}$. When $L \Sigma_{P} / G_{P}, L \Sigma_{O} / G_{O}$ are orientable, the choice of liftings does not matter. We shall also omit specifying the class $[v]$ in $\mathbb{A}_{(y,[v])}=: \mathbb{A}_{y}$, when the precise choice is immaterial.

For the symplectic Floer theory discussed in this article, this means replacing the deformation operators by their conjugates by trivializations 
of $u^{*} K$, namely $\Phi_{u}$ (cf. the definition of $\mathbb{E}_{u}, \mathbb{D}_{u}$ in Section 7.1.5). We write $(f)_{\Phi}:=\Phi_{u *} f$, e.g., $f=u^{\prime}$ for $u \in \mathcal{B}_{P}$ or $\mathcal{B}_{O}$.

The families of operators considered in the rest of this subsection will always be subfamilies of various versions of $\Sigma_{P}, \Sigma_{O}$. Thus, we shall refer to the correlation and relative signs of orientations of determinant lines, or mutual co-orientation of K-models without specifying the family.

The following consistency conditions on the choice of liftings will be assumed in the following discussion:

(a) for a subfamily $U \subset \mathcal{B}$, the lifting $\tilde{m}$ : $U \rightarrow \Sigma$ is continuous;

(b) the liftings are "coherent" in the sense that they are consistent with gluing.

7.3.2. Signs for $\mathbb{T}_{P, \text { hs-s }}$. To verify the sign in (I.30), we need to examine oriented K-models for the gluing theorem, Proposition 6.1.1 (a). Let $\left(J^{\Lambda}, X^{\Lambda}\right)$ be an admissible $(J, X)$-homotopy, for any $\mathbf{x}_{1}, \mathbf{x}_{2} \in \aleph_{\Lambda}$ and $R_{0} \gg 1$, the (omitted) proof of Proposition 6.1.1 (a) defines a gluing map

$\mathrm{Gl}_{P, \mathrm{hs}}\left(\mathbf{x}_{1}, \mathbf{x}_{2} ; \Re\right): \mathbb{T}_{P, \mathrm{hs}-\mathrm{s}}\left(\mathbf{x}_{\mathbf{1}}, \mathbf{x}_{\mathbf{2}} ; \Re\right) \times\left(R_{0}, \infty\right) \rightarrow \mathcal{M}_{P}^{\Lambda, 2}\left(\mathbf{x}_{1}, \mathbf{x}_{2} ; \mathrm{wt}_{-\mathrm{y}, e_{\mathcal{P}}} \leq \Re\right)$.

Let $\left(\lambda_{0}, \hat{u}\right) \in \hat{\mathcal{M}}_{P}^{\Lambda, 0}\left(\mathbf{x}, \mathbf{y} ; J^{\Lambda}, X^{\Lambda}\right)$ be a handleslide. Without loss of generality, assume $\lambda_{0}=0$. Let $\mathbf{q}, \mathbf{z} \in \aleph_{\Lambda}$ be of indices ind $\mathbf{y}+1$ and ind $\mathbf{y}-1$, respectively. Let

$$
\hat{v}_{-} \in \hat{\mathcal{M}}_{P}^{0}\left(q_{0}, x_{0} ; J_{0}, X_{0}\right), \quad \hat{v}_{+} \in \hat{\mathcal{M}}_{P}^{0}\left(y_{0}, z_{0} ; J_{0}, X_{0}\right)
$$

and $v_{-}, v_{+}, u$ be centered representatives of $\hat{v}_{-}, \hat{v}_{+}, \hat{u}$, respectively. Let

$$
w_{\#-}(R)=v_{-} \#_{R} u, \quad w_{\#+}(R)=u \#_{R} v_{+}
$$

be the pregluings defined in Section 1.2.2, and let

$$
\begin{aligned}
&\left(\lambda_{-}(R), w_{-}(R)\right):=\mathrm{Gl}_{P, \mathrm{hs}}(\mathbf{q}, \mathbf{y} ; \Re)\left(\left\{\hat{v}_{-}, \hat{u}\right\}, R\right), \\
&\left(\lambda_{+}(R), w_{+}(R)\right):=\mathrm{Gl}_{P, \mathrm{hs}}(\mathbf{x}, \mathbf{z} ; \Re)\left(\left\{\hat{u}, \hat{v}_{+}\right\}, R\right) .
\end{aligned}
$$

be the images of the gluing map obtained by further perturbing $w_{\#-}(R)$ and $w_{\#+}(R)$, respectively. To simplify notation, we shall omit $R$ when there is no danger of confusion.

Lemma. Let $u, v_{ \pm},\left(\lambda_{ \pm}, w_{ \pm}\right)$be as above. Then

$$
\begin{aligned}
& (1)-\operatorname{sign}\left(\lambda_{-}\right) \operatorname{sign}\left(w_{-}\right)=\operatorname{sign}\left(v_{-}\right) \operatorname{sign}(u) \\
& (2) \operatorname{sign}\left(\lambda_{+}\right) \operatorname{sign}\left(w_{+}\right)=\operatorname{sign}(u) \operatorname{sign}\left(v_{+}\right) .
\end{aligned}
$$

Proof. We shall focus on case (1) below, since case (2) is entirely parallel. According to Sections 7.1.3, 7.1.5, and the choice of coherent orientation, we have the oriented K-models: 
$(\mathrm{i} \pm)\left[\hat{\mathbb{E}}_{\left(0, w_{\# \pm}\right)}: \hat{K}_{\# \pm} \rightarrow C_{\# \pm}\right]$, where

$$
\begin{gathered}
\hat{K}_{\#-}=-\mathbb{R} \oplus\left(\text { ker } \mathbb{E}_{v_{-}} \#_{R} \text { ker } \mathbb{E}_{u}\right), \quad C_{\#-}=* \#_{R} \text { coker } \mathbb{E}_{u} ; \\
\hat{K}_{\#+}=\mathbb{R} \oplus\left(\operatorname{ker} \mathbb{E}_{u} \#_{R} \text { ker } \mathbb{E}_{v_{+}}\right), \quad C_{\#+}=\text { coker } \mathbb{E}_{u} \#_{R} * .
\end{gathered}
$$

(ii) $\left[\hat{\mathbb{E}}_{(0, u)}: \mathbb{R} \oplus\left(\right.\right.$ ker $\left.\mathbb{E}_{u}\right) \rightarrow$ coker $\left.\mathbb{E}_{u}\right]$.

Since $u$ is by assumption a nondegenerate element of $\mathcal{M}_{P}^{\Lambda, 1}$, the standard oriented K-model for $\hat{\mathbb{E}}_{(0, u)}$ may be viewed as a reduction of the oriented $\mathrm{K}$-model (ii) by $-\mathbb{R}$, taking

$$
\begin{aligned}
& \text { coker } \mathbb{E}_{u}=-\mathbb{R}\left(Y_{(0, u)}\right)_{\Phi} \quad \text { and } \\
& \text { ker } \mathbb{E}_{u}=\operatorname{ker} \hat{\mathbb{E}}_{(0, u)}=\operatorname{sign}(u) \mathbb{R}\left(u^{\prime}\right)_{\Phi}
\end{aligned}
$$

as oriented spaces. (Recall that $Y_{(0, u)}$ is a cutoff version of $\partial_{\lambda} \mathcal{V}_{\lambda}$ appearing in the definition of $\hat{\mathbb{E}}_{(0, u)}$, cf. I.6.1.5.)

Next, decompose $\hat{K}_{\#-}$ into the direct sum of the ordered triple of oriented subspaces

$$
* \oplus\left(\text { ker } \mathbb{E}_{v_{-}} \#_{R} *\right), \quad \mathbb{R} \oplus *, \quad \text { and } * \oplus\left(* \#_{R} \text { ker } \mathbb{E}_{u}\right) .
$$

By Lemma 1.2.4 (2), for large $R$, the restriction of $\Pi_{C_{\#-}} \hat{\mathbb{E}}_{\left(0, w_{\#-}\right)}$ to the first and last subspaces are small, while its restriction to the second subspace approximates the multiplication by $\Pi_{\text {coker }} \mathbb{E}_{u} \tilde{Y}$ (under the natural identification of the domain and range spaces), where $\tilde{Y}$ is another cutoff version of $\partial_{\lambda} \mathcal{V}_{\lambda}$ which agrees with $\left(Y_{(0, u)}\right)_{\Phi}$ except in the region where $s \ll-1$. Let $Y_{\nu}:=\nu\left(Y_{(0, u)}\right)_{\Phi}+(1-\nu) \tilde{Y}$ for $\nu \in[0,1]$, and $\hat{\mathbb{E}}_{\nu}$ be the rank-1 stabilization of $\mathbb{E}_{u}$ by multiplication by $Y_{\nu}$. By the surjectivity of $\partial_{s}+\mathbb{A}_{y}$ and $\hat{\mathbb{E}}_{0}=\mathbb{E}_{(0, u)}$, and an excision argument (as outlined in Section 1.2.5), $\hat{\mathbb{E}}_{\nu}$ has uniformly bounded right inveres. Thus, we may conclude that $\Pi_{\text {coker }} \mathbb{E}_{u} \tilde{Y}$, and hence also $\Pi_{C_{\#-}} \hat{\mathbb{E}}_{\left(0, w_{\#-}\right)}||_{\mathbb{R} \oplus *}$, are positive of $O(1)$. This implies that the reduction of the oriented $\mathrm{K}$-model $(\mathrm{i}-$ ) by $-\mathbb{R} \oplus *$ is equivalent to the standard oriented $\mathrm{K}$-model of $\hat{\mathbb{E}}_{\left(0, w_{\#-}\right)}$, which is in turn equivalent to the standard oriented $\mathrm{K}$-model of $\hat{\mathbb{E}}_{\left(\lambda_{-}, w_{-}\right)}$, due to the the proximity between $w_{-}$and $w_{\#-}$. In other words, the projection

$$
\Pi_{K_{\#-}} \text { : ker } \hat{\mathbb{E}}_{\left(\lambda_{-}, w_{-}\right)} \rightarrow \operatorname{ker} \mathbb{E}_{v_{-}} \#{ }_{R} \operatorname{ker} \hat{\mathbb{E}}_{(0, u)}=: K_{\#-} .
$$

is an orientation-preserving isomorphism. We have the following ordered bases compatible with the former and latter oriented spaces above:

$$
\begin{aligned}
& \left\{\operatorname{sign}\left(w_{-}\right)\left(0, w_{-}^{\prime}\right),\left(\partial_{R} \lambda_{-}\right)^{-1}\left(\partial_{R} \lambda_{-}, \partial_{R} w_{-}\right)\right\}, \\
& \left\{\operatorname{sign}\left(v_{-}\right)\left(0, v_{-}^{\prime}\right), \operatorname{sign}(u)\left(0, u^{\prime}\right)\right\} .
\end{aligned}
$$


Observing that $\operatorname{sign}\left(\partial_{R} \lambda_{-}\right)=-\operatorname{sign}\left(\lambda_{-}\right)$, and recalling the description of $\Pi_{\iota_{\#}^{j} K_{J}}$ in Section 1.2.4, one finds that with respect to these bases, $\Pi_{K_{\#-}}$ is a matrix of the form

$\left(\begin{array}{cc}C_{1} \operatorname{sign}\left(w_{-}\right) \operatorname{sign}\left(v_{-}\right) & C_{1}^{\prime} \operatorname{sign}\left(\lambda_{-}\right) \operatorname{sign}\left(v_{-}\right) \\ C_{2} \operatorname{sign}\left(w_{-}\right) \operatorname{sign}(u) & -C_{2}^{\prime} \operatorname{sign}\left(\lambda_{-}\right) \operatorname{sign}(u)\end{array}\right) \quad$ for $C_{1}, C_{1}^{\prime}, C_{2}, C_{2}^{\prime} \in \mathbb{R}^{+}$.

Equation (110.1) follows from the requirement that this matrix has positive determinant.

7.3.3. Signs for $\mathbb{T}_{O \text {,hs-s }}$. To verify the sign in (I.33), we examine the oriented K-model for the gluing theorem Proposition 6.1.1 (b). Let $y, u$ be as in Section 7.3.2, but now assume that the handleslide $u$ is of Type II, namely, $x=y$. Let $w_{\#}(R)=u_{\# R}$ be the glued orbit introduced in Section 1.2.2, and let $(\lambda(R),(T(R), w(R))):=\mathrm{Gl}_{O, \mathrm{hs}}(\hat{u}, R)$ be the image of the gluing map obtained by perturbing $w_{\#}(R)$, where $\mathrm{Gl}_{O \text {,hs }}: \mathbb{T}_{O, \text { hs-s }}(\Re) \times\left(R_{0}, \infty\right) \rightarrow$ $\hat{\mathcal{M}}_{O}^{\Lambda, 1}\left(\mathrm{wt}_{-y, e \mathcal{P}} \leq \Re\right)$ is the gluing map in the (omitted) proof of Proposition 6.1.1 (b).

Lemma. In the above notation, $\operatorname{sign}(w)=(-1)^{\text {ind } y} \operatorname{sign}(\lambda) \operatorname{sign}(u)$.

Proof. According to Corollary 7.2.4, $\operatorname{sign}(w)=(-1)^{\text {ind } y}\left[\left(w^{\prime}\right)_{\Phi}\right] / \operatorname{ker}^{\mathfrak{o}_{y}} \tilde{\mathbb{D}}_{(T, w)}$, where $\operatorname{ker}^{\mathfrak{o}_{y}} \tilde{\mathbb{D}}_{(T, w)}$ denotes ker $\tilde{\mathbb{D}}_{(T, w)}$ endowed with the orientation correlated to the canonical orientation of $\tilde{\mathbb{D}}_{\mathbb{A}_{y}, T}$. We compute $\left[\left(w^{\prime}\right)_{\Phi}\right] / \operatorname{ker}^{\mathfrak{o}_{y}} \tilde{\mathbb{D}}_{(T, w)}$ in two steps.

Step 1. The relative sign $\left[\left(w^{\prime}\right)_{\Phi}\right] / \operatorname{ker}^{\mathfrak{o}_{y}} \mathbb{D}_{(\lambda, w)}$. Let $\dot{D}_{(\lambda, w)}$ be the rank-1 stabilization of $D_{w}$ defined by

$$
\dot{D}_{(\lambda, w)}(\alpha, \xi)=\alpha \partial_{\lambda} \mathcal{V}_{\lambda}(w)+D_{w} \xi .
$$

Performing cyclic gluing to the oriented K-model $\left[\hat{\mathbb{E}}_{(\lambda, u)}: \mathbb{R} \oplus \operatorname{ker} \mathbb{E}_{u} \rightarrow\right.$ coker $\mathbb{E}_{u}$ ], we obtain an oriented $\mathrm{K}$-model for $\mathbb{D}_{w_{\#}}$, a rank-1 stabilization of $\mathbb{D}_{w_{\#}}$ by multiplication with a cutoff version of $\partial_{\lambda} \mathcal{V}_{\lambda}\left(w_{\#}\right)$. The argument in Section 7.3.2 shows that a reduction of this oriented K-model by $\mathbb{R}$ is equivalent to a standard K-model for $\mathbb{D}_{w_{\#}}$, which is in turn equivalent to a standard K-model for $\mathbb{D}_{(\lambda, w)}$. Moreover, according to the continuity of gluing homomorphisms and Lemma 7.2.2, the orientation of this standard $\mathrm{K}$-model is correlated to the canonical orientation of $\tilde{\mathbb{D}}_{\mathbb{A}_{y}, T}$. In other words,

$$
\left[\left(w^{\prime}\right)_{\Phi}\right] / \operatorname{ker}^{\mathfrak{o}_{y}} \mathbb{D}_{(\lambda, w)}=\operatorname{sign}(u) .
$$

Step 2. The relative sign $\operatorname{ker}^{\boldsymbol{o}_{y}} \dot{D}_{(\lambda, w)} / \operatorname{ker}^{\mathfrak{o}_{y}} \tilde{D}_{(T, w)}$. Notice that the operators $\dot{D}_{(\lambda, w)}, \tilde{D}_{(T, w)}$ have a common stabilization, namely $\hat{D}_{(\lambda,(T, w))}=$ $\left(\partial_{\lambda} \mathcal{V}_{\lambda},\left(-w^{\prime} / T, D_{w}\right)\right)$. The 2-dimensional space ker $\hat{D}_{(\lambda,(T, w))}$ is spanned by 
$\left\{\partial_{R}(\lambda,(T, w)),\left(0,\left(0, w^{\prime}\right)\right)\right\}$. This means $\Pi_{\text {coker } D_{w}}\left(\partial_{R} \lambda \partial_{\lambda} \mathcal{V}_{\lambda}+\partial_{R} T\left(-w^{\prime} / T\right)\right)$ $=0$, and hence the relative sign is computed by

$$
\operatorname{ker}^{\mathfrak{o}_{y}} \dot{D}_{(\lambda, w)} / \operatorname{ker}^{\boldsymbol{o}_{y}} \tilde{D}_{(T, w)}=-\operatorname{sign}\left(\partial_{R} \lambda\right) / \operatorname{sign}\left(\partial_{R} T\right)=\operatorname{sign}(\lambda) .
$$

Finally, the Lemma is obtained taking the product of the relative signs obtained in Steps 1 and 2 above with $(-1)^{\text {ind } y}$.

7.3.4. Signs for $\mathbb{T}_{P, \mathrm{db}}$. To verify the signs in (I.29), we need to analyze the orientation of the K-model for the gluing theorem Proposition 2.1 (a). Let $\lambda, u_{0}, \ldots, u_{k+1}$ be as in Section 2.2 , and let $(\lambda, w)$ be the image of $\left(\left\{u_{0}, u_{1}, \ldots, u_{k+1}\right\}, \lambda\right)$ under the gluing map defined in Section 4.1.4.

Lemma. Under the assumptions in Section 2.1 and in the above notation,

$$
\operatorname{sign}(w)=(-1)^{k+1} \prod_{i=0}^{k+1} \operatorname{sign}\left(u_{i}\right) .
$$

Proof. As explained earlier in this section, since we work with the ordinary Sobolev norms instead of the complicated polynomially weighted ones, it is convenient to replace the delicate pregluing $w_{\chi}$ defined in Section 2.2 by ordinary glued trajectories or orbits. Choose $R_{i}, R_{i}^{\prime}, i \in\{1, \ldots, k+1\}$ and $L$ appropriately so that:

$$
w_{\#}:=\tau_{L}\left(u_{0} \#_{R_{1}} \bar{y} \#_{R_{1}^{\prime}} u_{1} \#_{R_{2}} \cdots \#_{R_{k+1}^{\prime}} u_{k+1}\right)
$$

is pointwise close to $w$ and $w_{\chi}$ : more precisely, $w_{\#}(s), w(s), w_{\chi}(s)$ are $C_{\epsilon^{-}}$-close to each other $\forall s$, and

$$
w_{\chi}\left(\gamma_{u_{i}}^{-1}(0)\right)=w_{\#}\left(\gamma_{u_{i}}^{-1}(0)\right)
$$

As explained in Sections 7.2.1 and 7.2.3, the deformation operator in $\Sigma_{P}$ corresponding to $w_{\#}$ is:

$$
\mathbb{E}_{w_{\#}}^{\sigma}:=\mathbb{E}_{u_{0}}^{[0, \sigma]} \#_{R_{1}} \mathbb{E}_{\bar{y}}^{[\sigma,-\sigma]} \#_{R_{1}^{\prime}} \mathbb{E}_{u_{1}}^{[-\sigma, \sigma]} \#_{R_{2}} \mathbb{E}_{\bar{y}}^{[\sigma,-\sigma]} \#_{R_{2}^{\prime}} \cdots \#_{R_{k}^{\prime}} \mathbb{E}_{u_{k+1}}^{[-\sigma, 0]}
$$

for a small $\sigma>0$. Let $\overline{\mathbf{e}}_{y}^{\sigma}(s):=\varsigma^{-\sigma, \sigma}(s) \mathbf{e}_{y}$, and recall that

$$
\text { ker } \mathbb{E}_{\bar{y}}^{[-\sigma, \sigma]}=\text { coker } \mathbb{E}_{\bar{y}}^{[\sigma,-\sigma]}=\mathbb{R} \overline{\mathbf{e}}_{y}^{\sigma} ; \quad \text { coker } \mathbb{E}_{\bar{y}}^{[-\sigma, \sigma]}=\operatorname{ker} \mathbb{E}_{\bar{y}}^{[\sigma,-\sigma]}=* .
$$

Then by Lemma 1.2.4, we have the following oriented K-model for $\mathbb{E}_{w_{\#}}$ compatible with the coherent orientation:

$$
\begin{gathered}
{\left[\mathbb{E}_{w_{\#}}^{\sigma}: K_{\#} \rightarrow C_{\#}\right], \quad \text { where } C_{\#}=* \#_{R_{1}} \mathbb{R}_{\mathbf{e}_{y}^{\sigma}} \#_{R_{1}^{\prime}} * \cdots \#_{R_{k}^{\prime}} *,} \\
K_{\#}=(-1)^{k+1} \operatorname{ker} \mathbb{E}_{u_{0}}^{[0, \sigma]} \#_{R_{1}} * \#_{R_{1}^{\prime}} \operatorname{ker} \mathbb{E}_{u_{1}}^{[-\sigma, \sigma]} \#_{R_{2}} * \#_{R_{2}^{\prime}} \cdots \#_{R_{k}^{\prime}} \operatorname{ker} \mathbb{E}_{u_{k+1}}^{[-\sigma, 0]}
\end{gathered}
$$

On the other hand, in Section 3, we constructed the following K-model:

$$
\left[\mathbb{E}_{w_{\chi}}: K_{\chi} \rightarrow C_{\chi}\right]=\left[\mathbb{R} \mathfrak{e}_{u_{0}} \oplus \cdots \oplus \mathbb{R} \mathfrak{e}_{u_{k+1}} \rightarrow \mathbb{R} \mathfrak{f}_{1} \oplus \cdots \oplus \mathbb{R} \mathfrak{f}_{k+1}\right]
$$


$\left(\mathbb{E}_{w_{\chi}}\right.$ is now considered as an operator between ordinary Sobolev spaces. As remarked before, the polynomially weighted spaces are commensurate with the ordinary Sobolev spaces, and we do not need uniform boundedness of right inverses in this section. We have also suppressed the subscript $\Phi$ and written $\mathfrak{e}_{u_{i}}=\left(\mathfrak{e}_{u_{i}}\right)_{\Phi}, \mathfrak{f}_{j}=\left(\mathfrak{f}_{j}\right)_{\Phi}$ above for simplicity.)

Using the descriptions of $\Pi_{K_{\chi}}$ and $\Pi_{C_{\chi}}$ given in Section 1.2.4 and Lemma 4.1 .1 and the proximity between $\mathbb{E}_{w_{\#}}^{\sigma}$ and $\mathbb{E}_{w_{\chi}}$, one may easily check that the oriented $\mathrm{K}$-model $\left[K_{\#} \rightarrow C_{\#}\right]$ is equivalent to

$$
\left[(-1)^{k+1} \prod_{i=0}^{k+1} \operatorname{sign}\left(u_{i}\right) K_{\chi} \rightarrow C_{\chi}\right],
$$

implying that the latter is also compatible with the coherent orientation.

Next, observe that $\left(w^{\prime}\right)_{\Phi}$ projects positively to all $\mathfrak{e}_{u_{i}}$. This, together with the form of $\left.\Pi_{C_{\chi}} \mathbb{E}_{\chi}\right|_{K_{\chi}}$ given in Lemma 4.1 .3 (b), implies that the reduction of the above oriented K-model,

$$
\left[(-1)^{k+1} \prod_{i=0}^{k+1} \operatorname{sign}\left(u_{i}\right) \mathbb{R}\left(w^{\prime}\right)_{\Phi} \rightarrow *\right],
$$

is equivalent to the standard oriented $\mathrm{K}$-model for $\mathbb{E}_{w_{\chi}}$, which is in turn equivalent to the standard oriented $\mathrm{K}$-model for $\mathbb{E}_{w}$, due to the proximity between $w$ and $w_{\chi}$. These observations immediately imply the lemma.

7.3.5. Signs for $\mathbb{T}_{O, \mathrm{db}}$. To verify the sign in (I.32), we examine the orientation of the K-model in the proof of Proposition 2.1 (b). Let $\left\{\hat{u}_{1}, \ldots, \hat{u}_{k}\right\}$ be a broken orbit, $u_{i}$ be the centered representative of $\hat{u}_{i}$, and $(\lambda,(T, w))$ be the image of $\left(\left\{\hat{u}_{1}, \ldots, \hat{u}_{k}\right\}, \lambda^{\prime}\right)$ under the gluing map defined in Section 4.3.1.

Lemma. Under the assumptions in Section 2.1 and in the above notation,

$$
\operatorname{sign}(w)=(-1)^{\text {ind }-y+k} \prod_{i=1}^{k} \operatorname{sign}\left(u_{i}\right) .
$$

Proof. As argued in the proof of Lemma 7.3.3,

$$
\operatorname{sign}(w)=(-1)^{\text {ind }_{-} y} \operatorname{sign}(\lambda)\left(w^{\prime}\right)_{\Phi} / \operatorname{ker}^{\mathfrak{o}_{y-}} \mathbb{\mathbb { D }}_{(\lambda, w)},
$$

where the superscript $\mathfrak{o}_{y-}$ indicates the orientation correlated with the canonical orientation of $\tilde{\mathbb{D}}_{\mathbb{A}_{y}+\sigma, T}$. (Recall the definition ind $y=\operatorname{ind}\left(\mathbb{A}_{y}+\right.$ $\sigma)$.) According to the assumption of Section 2.1, $\operatorname{sign}(\lambda)=1$.

Instead of working with the standard K-model for $\tilde{\mathbb{D}}_{\mathbb{A}_{y}+\sigma, T}$, we find it easier to work with the following mutually co-oriented K-model: $\left[\mathbb{D}_{y}\right.$ : $\left.-\left(\mathbf{e}_{y}\right)_{\Phi} \rightarrow *\right]$, where $\mathbb{D}_{y}$ is the stabilization of $\mathbb{D}_{\mathbb{A}_{y}, T}$ by multiplication with $\left(\mathbf{e}_{y}\right)_{\Phi}$. To see that they are indeed co-oriented, observe that the interpolation 
between them, $\mathbb{D}_{\nu}=\left((1-\nu)\left(\mathbf{e}_{y}\right)_{\Phi}, \mathbb{D}_{\mathbb{A}_{y}+\nu \sigma, T}\right)$, is surjective $\forall \nu \in[0,1]$ and has the following continuous basis for the kernel: $\xi_{\nu}:=\left(\nu \sigma,-(1-\nu)\left(\mathbf{e}_{y}\right)_{\Phi}\right)$.

We now consider mutually co-oriented K-models for two operators approximating $\mathbb{D}_{(\lambda, w)}$ and $\mathbb{D}_{y}$, respectively. The proximity of the operators implies that these K-models also form mutually co-oriented K-models for to $\mathbb{D}_{(\lambda, w)}$ and $\mathbb{D}_{y}$, respectively. Choose an glued orbit

$$
w_{\#}=\tau_{L}\left(\bar{y} \#_{R_{1}} u_{1} \#_{R_{1}^{\prime}} \bar{y} \#_{R_{2}} \cdots \#_{R_{k}} u_{k} \#_{R_{k}^{\prime}}\right)
$$

appproximating $w$ and $w_{\chi}$ pointwise in the sense of Section 7.3.4, and let

$$
\begin{aligned}
\mathbb{D}_{w \#}^{\sigma} & :=\left(\partial_{\lambda} \mathcal{V}_{\lambda}\left(w_{\#}\right), \mathbb{E}_{\bar{y}}^{[\sigma,-\sigma]} \#_{R_{1}} \mathbb{E}_{u_{1}}^{[-\sigma, \sigma]} \#_{R_{1}^{\prime}} \mathbb{E}_{\bar{y}}^{[\sigma,-\sigma]} \#_{R_{2}} \mathbb{E}_{u_{2}}^{[-\sigma, \sigma]} \cdots \mathbb{E}_{u_{k}}^{[-\sigma, \sigma]} \#_{R_{k}^{\prime}}\right) \\
\mathbb{D}_{y \#}^{\sigma} & :=\left(-\left(\mathbf{e}_{y}\right)_{\Phi}, \mathbb{E}_{\bar{y}}^{[\sigma,-\sigma]} \#_{R_{1}} \mathbb{E}_{\bar{y}}^{[-\sigma, \sigma]} \#_{R_{1}^{\prime}} \mathbb{E}_{\bar{y}}^{[\sigma,-\sigma]} \#_{R_{2}} \mathbb{E}_{\bar{y}}^{[-\sigma, \sigma]} \cdots \mathbb{E}_{\bar{y}}^{[-\sigma, \sigma]} \#_{R_{k}^{\prime}}\right) .
\end{aligned}
$$

Since $\left[\mathbb{E}_{u_{i}}^{[-\sigma, \sigma]}: \operatorname{ker} E_{u_{i}}^{[-\sigma, \sigma]} \rightarrow *\right]$ and $\left[\mathbb{E}_{\bar{y}}^{[-\sigma, \sigma]}: \mathbb{R}_{\bar{y}}^{\sigma} \rightarrow *\right]$ are mutually cooriented K-models (by coherent orientation), the continuity of gluing homorphisms and stabilization imply that we have the mutually co-oriented Kmodels $\left[\mathbb{D}_{w \#}^{\sigma}: \hat{K}_{w \#} \rightarrow C_{\#}\right]^{\mathfrak{g l}},\left[\tilde{D}_{y \#}^{\sigma}: \hat{K}_{y \#} \rightarrow C_{\#}\right]^{\mathfrak{g l}}$, where

$$
\begin{aligned}
& \hat{K}_{w \#}=(-1)^{k+1} \mathbb{R} \oplus\left(* \#_{R_{1}} \operatorname{ker} \mathbb{E}_{u_{1}}^{[-\sigma, \sigma]} \#_{R_{1}^{\prime}} * \#_{R_{2}} \operatorname{ker} \mathbb{E}_{u_{2}}^{[-\sigma, \sigma]} \cdots \operatorname{ker} \mathbb{E}_{u_{k}}^{[-\sigma, \sigma]} \#_{R_{k}^{\prime}}\right) \\
& C_{\#}=\left(\mathbb{R} \hat{\mathbf{e}}_{y}^{\sigma}\right) \#_{R_{1}} * \#_{R_{1}^{\prime}}\left(\mathbb{R} \hat{\mathbf{e}}_{y}^{\sigma}\right) \cdots * \#_{R_{k}^{\prime}} \text {, } \\
& \hat{K}_{y \#}=(-1)^{k+1} \mathbb{R} \oplus\left(* \#_{R_{1}}\left(\mathbb{R} \hat{\mathbf{e}}_{y}^{\sigma}\right) \#_{R_{1}^{\prime}} * \#_{R_{2}}\left(\mathbb{R} \hat{\mathbf{e}}_{y}^{\sigma}\right) \cdots\left(\mathbb{R} \hat{\mathbf{e}}_{y}^{\sigma}\right) \#_{R_{k}^{\prime}}\right) .
\end{aligned}
$$

Note that the orientation of these K-models is different from the gradingcompatible orientation or the $\mathfrak{o}_{y}$ - orientation. We call it the "glued orientation," indicated by the superscript $\mathfrak{g l}$ above.

We now compute the sign of $\left(w^{\prime}\right)_{\Phi}$ relative to the $\mathfrak{g l}$-orientation above. As in Section 7.3.4, this is done by comparing the glued K-model above to $\left[\mathbb{D}_{\left(\lambda, w_{\chi}\right)}: \hat{K}_{\chi} \rightarrow C_{\chi}\right]:=\left[\mathbb{R}_{\alpha} \oplus \mathbb{R}_{u_{1}} \oplus \cdots \oplus \mathbb{R e}_{u_{k}} \rightarrow \mathbb{R f}_{1} \oplus \cdots \oplus \mathbb{R f}_{k}\right]$, constructed previously in Section 4.3. In this case, we find $\left[\mathbb{D}_{w \#}^{\sigma}: \hat{K}_{w \#} \rightarrow\right.$ $\left.C_{w \#}\right]^{\mathfrak{g l}}$ equivalent to

$$
\left[\mathbb{D}_{\left(\lambda, w_{\chi}\right)}:(-1)^{k+1} \Pi_{i}\left(\operatorname{sign}\left(u_{i}\right)\right) \hat{K}_{\chi} \rightarrow C_{\chi}\right] .
$$

On the other hand, in Lemma 4.1 .3 (b), $\left.\Pi_{C_{\chi}} \mathbb{D}_{w_{\chi}}\right|_{\hat{K}_{\chi}}$ is computed in the bases $\left\{1, \mathfrak{e}_{u_{1}}, \ldots \mathfrak{e}_{u_{k}}\right\},\left\{\mathfrak{f}_{j}\right\}$ to be of the form:

$$
\left(\begin{array}{cccccc}
+ & - & 0 & \cdots & \cdots & + \\
+ & + & - & 0 & \cdots & 0 \\
+ & 0 & + & \ddots & \cdots & 0 \\
\vdots & 0 & 0 & \ddots & \ddots & \vdots \\
+ & 0 & \cdots & \cdots & + & -
\end{array}\right) \quad(+/- \text { denote positive/negative numbers. })
$$


modulo ignorable terms. Combining this with the fact that, in terms of the same basis,

we see that

$$
\Pi_{\hat{K}_{\chi}}\left(w^{\prime}\right)_{\Phi}=(0,+,+, \cdots,+)
$$

$$
\left[\left(w^{\prime}\right)_{\Phi}\right] / \operatorname{ker}^{\mathfrak{g l}} \mathbb{D}_{(\lambda, w)}=-\prod_{i=1}^{k} \operatorname{sign}\left(u_{i}\right) .
$$

Next, we need to find the relative sign between the $\mathfrak{g l}$ and $\mathfrak{o}_{y}$ orientations. For this purpose, we compute explicitly the form of the operator $\left.\Pi_{C_{\#}} \dot{\mathbb{D}}_{y \#}\right|_{\hat{K}_{y \#}}$. In terms of the bases $\left\{1, e_{1}, \ldots, e_{k},\right\}$ and $\left\{f_{j}\right\}$, where

$$
\begin{aligned}
e_{i} & :=* \#_{R_{1}} * \cdots * \#_{R_{i}} \overline{\mathbf{e}}_{y}^{\sigma} \#_{R_{i}^{\prime}} * \cdots * \#_{R_{k}^{\prime}} ; \\
f_{j}: & =* \#_{R_{1}} * \cdots * \#_{R_{j-1}^{\prime}} \overline{\mathbf{e}}_{y}^{\sigma} \#_{R_{j}} * \cdots * \#_{R_{k}^{\prime}},
\end{aligned}
$$

it has the following form:

$$
\left(\begin{array}{cccccc}
+ & + & 0 & \cdots & \cdots & - \\
+ & - & + & 0 & \cdots & 0 \\
+ & 0 & - & \ddots & \cdots & 0 \\
\vdots & \vdots & \ddots & \ddots & \ddots & \vdots \\
+ & 0 & \cdots & 0 & - & +
\end{array}\right) .
$$

Combining this with the facts that, in the same basis,

$$
\Pi_{\hat{K}_{y \#}}\left(-\left(\mathbf{e}_{y}\right)_{\Phi}\right)=(0,-,-, \cdots,-),
$$

we have by the proximity between $\mathbb{D}_{y \#}$ and $\mathbb{D}_{y}$ that

$$
\operatorname{sign}\left(\mathfrak{o}_{y-} / \mathfrak{g l}\right)=-\left[\left(\mathbf{e}_{y}\right)_{\Phi}\right] / \operatorname{ker}^{\mathfrak{g l} l} \dot{\mathbb{D}}_{y}=(-1)^{k+1} .
$$

The Lemma now follows by combining this with (112) and (111).

\section{References}

[1] Y.-J. Lee, Reidemeister torsion in Floer-Novikov theory and counting pseudoholomorphic tori, I, J. Symplectic Geom. 3 (2005), no. 2, 221-311.

[2] S. Donaldson, and P. Kronheimer, The geometry of four-manifolds, Oxford University Press, 1990.

[3] S. Donaldson, Floer homology groups in Yang-Mills theory, Cambridge University Press, Cambridge, 2002.

[4] A. Floer and H. Hofer, Coherent orientations for periodic orbit problems in symplectic geometry, Math. Z. 212 (1993), no. 1, 13-38.

[5] D. Salamon, Lectures on Floer homology, in Symplectic geometry and topology (Park City, UT, 1997), AMS 1999.

[6] A. Floer, Morse theory for Lagrangian intersections, J. Differential Geom. 28 (1988), no. $3,513-547$. 
[7] A. Floer, H. Hofer and D. Salamon, Transversality in elliptic Morse theory for the symplectic action, Duke Math. J 80 (1995), 251.

[8] Y.-J. Lee, Noncontractible periodic orbits, Gromov invariants, and Floer-theoretic torsions, math.SG/0308185.

Department of Mathematics

Purdue University

WEST LAFAYETTE, IN 47907

E-mail address: yjlee@math.purdue.edu

Partially supported by NSF-grant DMS-0333163. 